\title{
Synthesewege zu neuen Mehrkernkomplexen von Pyrazol-NHC-Hybridliganden mit Übergangs- metallen - mit Fokus auf Silber-Komplexe
}

\author{
Dissertation \\ zur Erlangung des mathematisch-naturwissenschaftlichen Doktorgrades \\ "Doctor rerum naturalium" \\ der Georg-August-Universität
}

vorgelegt von

Maria Georgiou-Smith geb. Georgiou

aus Edessa, Griechenland

Göttingen, 2010 
Referent: Prof. Dr. Franc Meyer

Korreferent: Prof. Dr. Dietmar Stalke

Tag der mündlichen Prüfung: 
The important thing is to understand what you're doing, rather than to get the right answer.

Tom Lehrer 



\section{Inhaltsverzeichnis}

I Allgemeiner Teil 1

1 Einleitung 3

1.1 Allgemeines über Carbene . . . . . . . . . . . . . . . 3

1.2 Eine Unterklasse: $N$-heterozyklische Carbene (NHCs) $\ldots \ldots \ldots$

1.3 Wichtige Fortschritte in der Carben-Chemie . . . . . . . . . 6

1.4 NHCs vs. klassische Carbene und ihre Komplexchemie . . . . . . 9

2 Grundlagen zur Synthese und Stand der Forschung 11

2.1 Ligandsynthese . . . . . . . . . . . . . . . . . . . 11

2.1.1 Pyrazol: Der Baustein für die Liganden . . . . . . . . . . . . 11

2.1.2 N-substituierte Imidazole: Die Seitenarme . . . . . . . . 12

2.2 Synthesemöglichkeiten von NHCs . . . . . . . . . . . . . 15

2.2.1 Im Fokus dieser Arbeit: Silber-NHC-Komplexe . . . . . . . 17

2.3 Anwendungsmöglichkeit: Katalyse . . . . . . . . . . . . . . 22

2.3 .1 NHC-Komplexe in der Katalyse . . . . . . . . . . . . . . 24

3 Zielsetzung 27

4 Ergebnisse und Diskussion $\quad 29$

4.1 Synthese der Pyrazol-NHC-Liganden . . . . . . . . . . . . . . . 29

4.2 Synthese der Komplexe . . . . . . . . . . . . . . . . . . . 36

4.2 .1 Silber-NHC-Komplexe . . . . . . . . . . . . . . . . 36

4.2 .2 Palladium-NHC-Komplexe . . . . . . . . . . . . . . . . 48

4.2 .3 Komplexe mit weiteren Metallen . . . . . . . . . . . . 56

4.3 Katalyse . . . . . . . . . . . . . . . . . . . . . . . . 59

5 Zusammenfassung $\quad 61$ 
II Experimenteller Teil $\quad 65$

6 Allgemeine Arbeitstechnik $\quad 67$

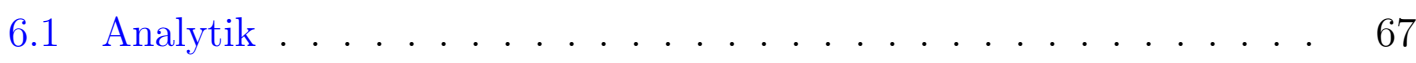

6.2 Verwendete Chemikalien . . . . . . . . . . . . . . . . . 68

6.3 Allgemeine Arbeitsvorschriften . . . . . . . . . . . . . . . 69

6.3.1 Synthese der Liganden als Hydrochloride . . . . . . . . . . . 69

6.3.2 Umsalzen der Liganden . . . . . . . . . . . . . . . . . . 74

6.3.3 Synthese von Silber-NHC-Komplexen . . . . . . . . . . . . 82

6.3.4 Synthese von Palladium-NHC-Komplexen . . . . . . . . . 88

6.3.5 Synthese von Nickel-NHC-Komplexen . . . . . . . . . . . . . 91

6.3.6 Palladium-katalysierte allylische Alkylierung . . . . . . . . . 92

$\begin{array}{lll}7 & \text { Kristallstrukturdaten } & 95\end{array}$

$\begin{array}{ll}\text { III Anhang } & 101\end{array}$

$\begin{array}{ll}\text { Literaturverzeichnis } & 103\end{array}$

$\begin{array}{ll}\text { Symbolverzeichnis } & 113\end{array}$

$\begin{array}{ll}\text { Abbildungsverzeichnis } & \mathbf{1 1 5}\end{array}$

$\begin{array}{ll}\text { Verzeichnis der Schemata } & 117\end{array}$ 


\section{Teil I}

Allgemeiner Teil 



\section{Kapitel 1}

\section{Einleitung}

\subsection{Allgemeines über Carbene}

Als Carbene bezeichnet man neutrale kohlenstoffhaltige Verbindungen, in denen ein Kohlenstoffatom zweifach gebunden ist und nur sechs Valenzelektronen besitzt. Dadurch verbleiben am Kohlenstoffatom zwei nichtbindende Elektronen. Je nachdem in welchen Orbitalen sich diese zwei nichtbindenden Elektronen befinden, unterscheidet man für die Carbene zwei verschiedene Elektronenkonfigurationen im Grundzustand. Wenn die beiden nichtbindenden Elektronen einen antiparallelen Spin besitzen und sich in einem gemeinsamen Orbital befinden, liegt ein SingulettZustand vor (Abbildung 1.1 (I)). Ein Orbital bleibt hierbei leer. Besetzen hingegen die zwei Elektronen unterschiedliche Orbitale mit parallelen Spins, handelt es sich um ein Triplett-Carben (Abbildung 1.1 (II)). [1]

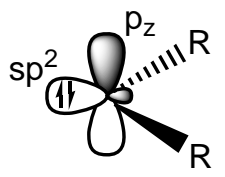

I

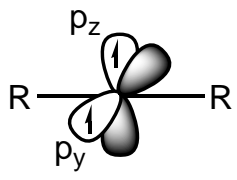

II

Abbildung 1.1: Struktur von Singulett- (I) und Triplett-Carbenen (II).

Über die Elektronenkonfiguration eines Carbens erhält man Information über dessen Geometrie, Stabilität sowie Reaktivität. Die Geometrie eines CarbenKohlenstoffatoms kann linear oder gewinkelt sein. Das einfachste denkbare Carben 
ist das Methylen : $\mathrm{CH}_{2}$ (für $\mathrm{R}=\mathrm{H}$; Abbildung 1.1 (II)), es weist eine lineare Geometrie auf. [2, 3] Das Kohlenstoffatom ist sp-hybridisiert und besitzt zwei entartete p-Orbitale $\left(\mathrm{p}_{x}, \mathrm{p}_{y}\right)$. Diesem Kohlenstoffatom wird ein Triplett-Zustand zugeordnet. Die meisten Carbene haben ein $\mathrm{sp}^{2}$-hybridisiertes Kohlenstoffatom mit einer nichtlinearen Struktur. Die beiden nichtbindenden Elektronen befinden sich entweder in dem $\sigma$ - oder dem $\mathrm{p}_{\pi}$-Orbital. Die Energiedifferenz zwischen dem $\sigma$ - und dem $\mathrm{p}_{\pi}$-Orbital ist entscheidend dafür welcher Grundzustand vorliegt. Ist diese in der Größenordnung von $2 \mathrm{eV}$, wird der Singulett-Zustand stabilisiert. [4, 5] Im Fall von kleineren energetischen Differenzen der Orbitale $(<1.5 \mathrm{eV})$ wird hingegen der Triplett-Zustand favorisiert. [5] Für die meisten Carbene ist experimentell eine gewinkelte Struktur mit Triplett-Grundzustand nachgewiesen.

Neben den oben erwähnten Faktoren für die Spinmultiplizität eines CarbenGrundzustandes spielen auch induktive elektronische Effekte eine große Rolle. [1] Trägt das Carben elektronenziehende Substituenten (-I-Effekt), wird der SingulettZustand stabilisiert. Durch die elektronenziehenden Substituenten wird das $\sigma$ Orbtial energetisch abgesenkt, während das $\mathrm{p}_{\pi}$-Orbital keine energetische Änderung erfährt. Dies bedeutet eine Vergrößerung der Energiedifferenz zwischen den beiden Orbitalen und resultiert in der Stabilisierung des Singulett-Zustands. Auf der anderen Seite stabilisieren elektronenschiebenden Substituenten (+I-Effekt) den Triplett-Zustand; die Energiedifferenz zwischen den beiden Orbitalen wird hier verringert.

Zusätzlich sind mesomere Effekte zu berücksichtigen. Der Einfluss der mesomeren Effekte ist wie folgt zu erklären: Trägt der Carben-Kohlenstoff beispielsweise zwei benachbarte Substituenten mit positivem mesomeren Effekt (+M-Effekt), so wird das $\mathrm{p}_{\pi}$-Orbital durch die Wechselwirkung mit ihren beiden freien Elektronenpaaren energetisch angehoben (Abbildung 1.2 (III)). Dies verursacht eine Vergrößerung der Energiedifferenz zwischen den Orbitalen und die gewinkelten SingulettKonfiguration für das Carben wird bevorzugt. [6] Sind jedoch zwei Substituenten mit einem negativen mesomeren Effekt (-M-Effekt) vorhanden (diese besitzen leere Orbitale), kann die Elektronendichte vom Carben auf die Heteroatome übertragen werden. Dadurch wird ein linearer Singulett-Zustand stabilisiert (Abbildung 1.2 (IV)). Ist sowohl eine Gruppe mit einem + M-Effekt als auch eine Gruppe mit einem -MEffekt in Nachbarschaft zum Carben vorhanden, wird das Carben als ein annähernd lineares Allen-artiges System formuliert (Abbildung $1.2(\mathbf{V})$ ). [7]

Singulett-Carbene besitzen auf Grund der vollständigen Besetzung des einen und der fehlenden Besetzung des anderen Orbitals ein nukleophiles oder elektrophiles Zentrum und können nukleophile und elektrophile Reaktionen eingehen. Liegt hin- 


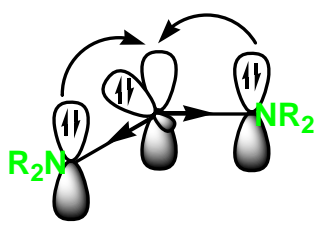

III

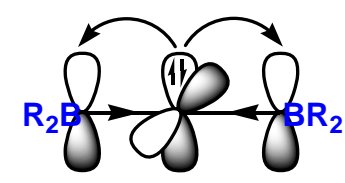

IV

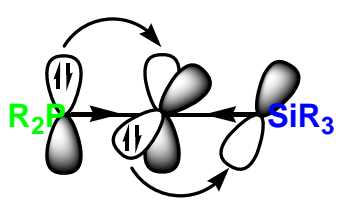

V

Abbildung 1.2: Einfluss von Substituenten mit induktiven und mesomeren Effekten.

gegen der Triplett-Zustand vor, reagieren die Carbene radikalisch, da sie zwei ungepaarte Elektronen haben und somit als Diradikale betrachtet werden.

\subsection{Eine Unterklasse: $N$-heterozyklische Carbene}

Die wohl am besten untersuchten Carbene sind die $N$-heterozyklischen Carbene (NHCs). Außer der Tatsache, dass die beiden $N$-Substituenten einen +M-Effekt mit sich bringen und somit ein gewinkeltes System mit SingulettElektronenkonfiguration stabilisieren (siehe Kapitel 1.1), ist eine zusätzliche Stabilisierung durch den aromatischen Heterozyklus möglich, in den das Carben integriert werden kann. In Abbildung 1.3 sind die möglichen Resonanzstrukturen dargestellt, bei denen der Doppelbindungscharakter der Verbindung deutlich wird.

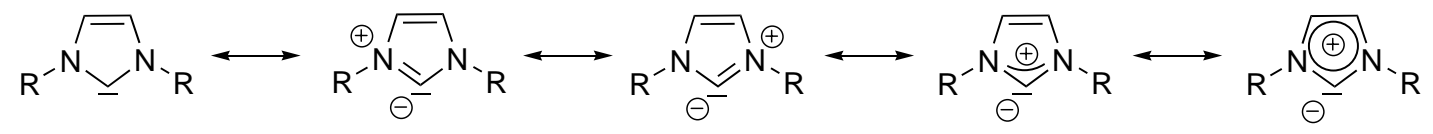

Abbildung 1.3: Mesomere Grenzstrukturen von Imidazolyl-2-ylidenen.

Auch sterische Effekte sind insofern von Bedeutung, als sperrige Substituenten Carbene kinetisch stabilisieren. [1] Je sterisch anspruchsvoller die Substituenten an den Stickstoffatomen sind, desto mehr wird der Triplett-Zustand stabilisiert. Allerdings spielen sterische Einflüsse eine nicht so bedeutende Rolle wie elektronische Effekte. Erstere können zur Stabilisierung beitragen und letztere sind für die Stabilisierung eines Carbens entscheidend. [2, 8] 


\subsection{Wichtige Fortschritte in der Carben-Chemie}

Carbene zählen zu den wichtigsten Verbindungen in der metallorganischen Chemie und sind seit langer Zeit bekannt. [3] Bereits 1835 unternahm Dumas den Versuch das einfachste Carben, das Methylen, zu isolieren (Schema 1.1). [9] Er betrachtete Methanol als ein aus Methylen und einem Äquivalent Wasser bestehendes Addukt. Sein Vorhaben war es, mit Hilfe von stark wasserentziehenden Mitteln wie Phosphorpentoxid oder Schwefelsäure Methylen zu erzeugen.

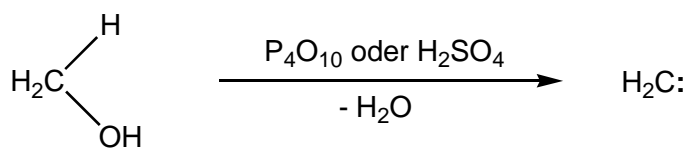

Schema 1.1: Erster Versuch Methylen aus Methanol zu isolieren.

Die Darstellung eines Methylen-Derivates, nämlich des Dichlormethylens, wurde 1862 von Geuther verfolgt. [10] Sein Ansatz basierte darauf, dass ein Carben aus einem relativ stabilen Molekül unter Eliminierung eines kleineren Moleküls synthetisiert werden kann (Schema 1.2). Bemerkenswerterweise gelingt diese Reaktion damals genau wie heute, mit dem Nachteil, dass das gebildete Dichlorcarben unter diesen Herstellungsbedingungen hochreaktiv ist.

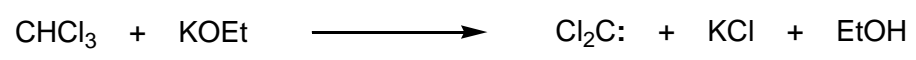

Schema 1.2: Darstellung eines intermediär auftretenden Methylen-Derivates.

Ein ähnliches Vorhaben äußerte Nef im Jahr 1897. [11] In seiner Theorie formulierte er das Methylen als den Grundbaustein der organischen Chemie und die bis dato durchgeführten Untersuchungen wiesen darauf hin, dass solche Verbindungen tatsächlich existieren könnten. Seine Theorie konnte jedoch nicht bestätigt werden. Der wesentliche Grund dafür war die hohe Reaktivität der Carbene, weshalb die Isolierung solcher Verbindungen lange Zeit nicht möglich war. Carbene konnten lediglich als Liganden in Komplexen dargestellt werden. So gelang es Tschugajeff 1925 den ersten mit Stickstoffatomen stabilisierten Carben-Ligand zu synthetisieren und in Form des Platin-Komplexes VI zu isolieren (Schema 1.3). [12]

Ende der fünfziger Jahre berichtete Wanzlick über die Synthese des $N$ heterozyklischen Carbens VIII durch die $\alpha$-Eliminierung von Chloroform aus Verbindung VII (Schema 1.4). [13] Allerdings gelang es ihm nicht dieses zu isolieren, sondern er erhielt die dimere Verbindung IX. Er postulierte somit ein Gleichgewicht 
<smiles></smiles><smiles></smiles>

VI

Schema 1.3: Isolierung des ersten mit Stickstoffatomen stabilisierten Platin-Carbenkomplexes VI.

zwischen dem Carbendimer (Tetraaminoethylen-Derivat) und dem entsprechenden Monomer. Diese Annahme wurde kurze Zeit später von Lemal widerlegt. [14] Eine Dimerisierung läge nicht vor.<smiles>ClC(Cl)(Cl)C1(c2ccccc2)NCCN1c1ccccc1</smiles>

VII<smiles>CCCCCCCC</smiles><smiles></smiles>

VIII<smiles>Pc1ccccc1</smiles>

IX

Schema 1.4: Darstellung des Tetraaminoethylen-Derivats IX durch $\alpha$-Eliminierung von VII.

Im Jahr 1968 tritt erstmals in der Literatur die Synthese $N$-heterozyklischer Carbene auf. Öfele [15] und Wanzlick et al. [16] berichteten unabhängig voneinander über Carben-Metallkomplexe mit Chrom bzw. Quecksilber als Metall. In Schema 1.5 sind diese Metallkomplexe dargestellt. Lappert und Mitarbeiter haben in den folgenden Jahren NHC-Metallkomplexe untersucht, dessen Synthese von elektronenreichen Olefinen ausging. [17, 18]

Den entscheidenden Durchbruch auf dem Gebiet der Carben-Chemie initiierte Arduengo [19], als er 1991 das erste stabile, freie $N$-heterozyklische Carben $\mathbf{X}$ isolierte (Schema 1.6). Die Entwicklung der eigentlichen metallorganischen CarbenChemie wurde somit angestoßen. Schon kurz darauf erkannte Herrmann, dass $N$ heterozyklische Carbene sich hervorragend als Liganden für die Darstellung von Übergangsmetallkomplexen eignen. [20] Somit eröffnete sich ein neues, weites Feld mit einer breiten Anwendungspalette in der Koordinationschemie. 

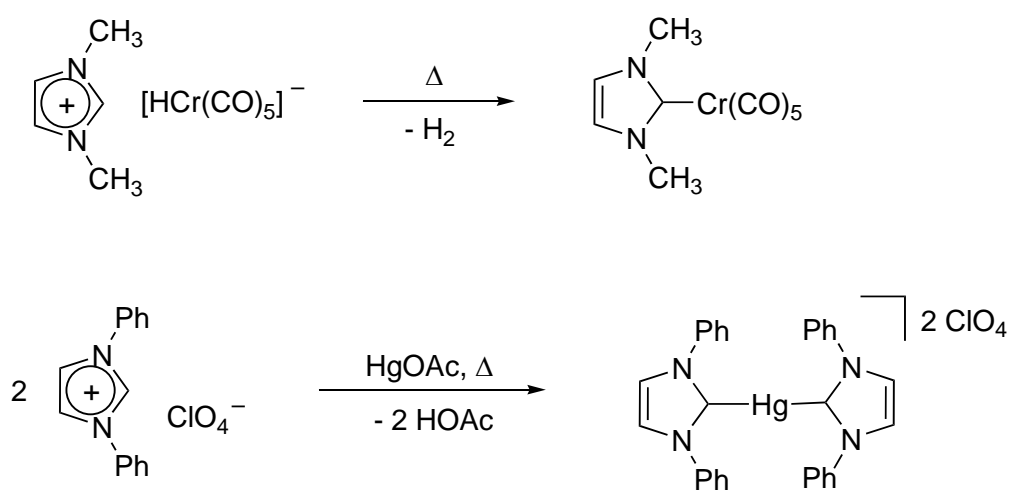

Schema 1.5: Carben-Metallkomplexe nach Öfele (oben) und Wanzlick (unten).
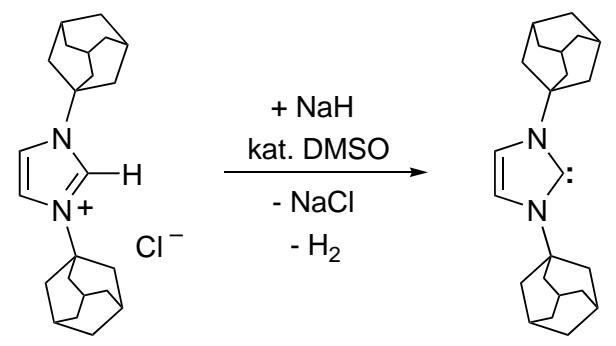

$\mathbf{X}$

Schema 1.6: Synthese des ersten von Arduengo isolierten stabilen $N$-heterozyklischen Carbens X.

Es sollte erwähnt werden, dass die Einführung von Carbenen in die metallorganische Chemie bereits im Jahr 1964 durch Fischer et al. erfolgte. [21] Ab diesem Zeitpunkt wurden die Bezeichnungen „Fischer-“ und „Schrock-Carbenkomplexe“ $[22,23]$, nach ihren Entdeckern genannt, in der Literatur eingeführt um die Carbene hauptsächlich nach ihrer Reaktivität, direkt tragenden Substituenten und der Oxidationsstufe des Metalls zu klassifizieren. In Abbildung 1.4 sind Beispiele dieser Verbindungsklassen angegeben. Die „Fischer-Carbenkomplexe“ enthalten ein spätes Übergangsmetall in niedriger Oxidationsstufe, so dass die 18 VE-Regel erfüllt ist. Der Carben-Kohlenstoff liegt im Singulett-Grundzustand vor und hat elektrophilen Charakter. Zumindest ein Substituent am carbenoiden Kohlenstoff ist ein Heteroatom. Im Gegensatz dazu bilden die klassischen „Schrock-Carbenkomplexe“ oder auch Alkylidenkomplexe stabile Komplexe mit frühen Übergangsmetallen in hoher Oxidationsstufe mit häufig weniger als 18 Valenzelektronen. Der carbenoide Kohlenstoff reagiert in der Regel nukleophil. [24] 


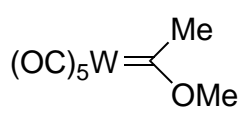

$\mathrm{Cp}_{2} \mathrm{Ti}=<_{\mathrm{H}}^{\mathrm{H}}$

Abbildung 1.4: Beispiel eines Fischer- (rechts) und eines Schrock-Carbenkomplexes (links).

\subsection{NHCs vs. klassische Carbene und ihre Komplexchemie}

$N$-heterozyklische Carbene können in chemischen Reaktionen hervorragend als Substrat fungieren, als Ligand eingesetzt werden oder auch die Rolle des Katalysators übernehmen. Sie zeigen einen ausgeprägten $\sigma$-Donorcharakter und werden mit Liganden verglichen, die über Heteroatome wie Phosphor, Stickstoff oder Sauerstoff koordinieren. Ihre Bindungseigenschaften zum Metall ähneln stark den von Phosphanen. Herrmann hat als erster die Ähnlichkeit zwischen NHCs und tertiären Phosphanen hinsichtlich ihrer elektronischen Eigenschaften aufgezeigt. [25] NHCs sind wesentlich bessere Donoren als Phosphane - eine Ausnahme stellen hierbei die sterisch anspruchsvollen adamantylsubstituierten NHC-Derivate dar, welche das Metallzentrum stark abschirmen, so dass die Zugänglichkeit zum Metall erschwert wird. NHC-Metallkomplexe weisen eine hohe Stabilität auf und werden deswegen bevorzugt im Liganden-Design für katalytische Systeme eingesetzt. Die Bindungslängen der Metall-Carben-Bindungen liegen im Bereich von Einfachbindungen. Deren $\pi$ Rückbindungsanteil kann laut theoretischen Berechnungen als gering betrachtet werden. [26, 27] Somit unterscheiden sich die Metall-Carben-Bindungsverhältnisse von NHCs deutlich von denen der klassischen Carbenliganden des „Schrock-“ und des „Fischer-Typs“. Dort liegen die Bindungen im Bereich von Doppelbindungen, was sich durch Rückbindungseffekte erklären lässt. Der Einfachbindungs-Charakter der NHCs erlaubt die freie Drehbarkeit um diese Bindung und resultiert in einer hohen Flexibilität, welche vorteilhaft für den Aufbau der Komplexe ist.

NHCs besitzen die Fähigkeit mit Metallen in sowohl hohen als auch niedrigen Oxidationsstufen Bindungen einzugehen. Aufgrund des starken $\sigma$-Donorcharakters sind diese Bindungen sehr stabil. Daraus resultiert eine hohe chemische und thermische Stabilität der NHC-Komplexe. Neben der stabilen Metall-Kohlenstoff-Bindung gehört die einfache Modifizierbarkeit des heterozyklischen Grundgerüstes zu den großen Vorteilen der NHC-Komplexe und ist ein weiterer Grund für ihren Einsatz als Liganden. So können unter anderem Substituenten, die chirale Information tragen, solche die zur einen effektiven Immobilisierung beitragen, oder auch Gruppen 
die eine Verbesserung der (Wasser)-Löslichkeit erzielen können, eingeführt werden. [28, 29] Ferner lassen sich mit NHCs Chelatliganden aufbauen, die außer der NHCEinheit noch andere Donoratomen tragen. So sind beispielsweise NHC,N- [30, 31] oder NHC,P-Chelatliganden [32, 33] erfolgreich synthetisiert worden, mit dem viele Metalle koordiniert wurden. Des Weiteren zeichnen sich NHCs durch ihre strukturelle Vielfalt aus. Durch Variation des sterischen Anspruchs an den Stickstoffatomen der NHCs sowie der elektronischen Eigenschaften des Heterozyklus an sich (Imidazol, Benzimidazol, Triazol u.a.) kann zum großen Teil die Koordinationssphäre auf das jeweilige Metall eingestellt werden. Auf diese Weise gelingt der Zugang zu zahlreichen Liganden und Komplexen.

Die NHCs zeichnen sich in Metallkomplexen durch eine hohe Luftstabilität aus und sind infolgedessen mit einer unkomplizierten Handhabung verbunden. Zu den Vorteilen zählen sicherlich auch die einfachen Synthesemöglichkeiten und das Arbeiten unter milden Reaktionsbedingungen. 


\section{Kapitel 2}

\section{Grundlagen zur Synthese und Stand der Forschung}

\subsection{Ligandsynthese}

In den letzten Jahren wurde eine Klasse von Pyrazol-NHC-Hybridliganden entwickelt und untersucht, die in der Lage sind gleichzeitig zwei Metalle zu binden. [34-37] Um zu den gewünschten Liganden zu gelangen, wird eine zentrale PyrazolEinheit mit $N$-substituierten Imidazol-Seitenarmen versehen. Von besonderem Interesse sind in der vorliegenden Arbeit Imidazole, die sperrige Substituenten wie tert-Butyl und 1-Adamantyl tragen. Durch die Modifizierung sowohl am Pyrazol als auch an den Seitenarmen ist eine Vielzahl an Liganden zugänglich.

\subsubsection{Pyrazol: Der Baustein für die Liganden}

Da 3,5-substituiertes Pyrazol den zentralen Baustein für die Ligandensynthese darstellt, soll dessen Synthese an dieser Stelle näher beschrieben werden.

Das 3,5-Bis(chlormethyl)-1-(tetrahydropyran-2-yl)-1 $H$-pyrazol (6) wird nach literaturbekannten Vorschriften synthetisiert. [38, 39] Hierbei wird das 3,5-Dimethylpyrazol (1) mit Kaliumpermanganat unter Einleitung von $\mathrm{CO}_{2}$ zum Monokaliumsalz der Pyrazol-3,5-dicarbonsäure (2) oxidiert. Dieses wird unter säurekatalytischer Einwirkung mit Methanol versetzt und reagiert zum 3,5Pyrazoldicarbonsäuremethylester Hydrochlorid (3a), welches im nächsten Schritt 
mit Lithiumaluminiumhydrid zum Dialkohol 4 reduziert wird. Die anschließende Reaktion mit Thionylchlorid führt zum 3,5-Bis(chlormethyl)-pyrazol Hydrochlorid (5). Die NH-Funktion des Pyrazols muss während der darauffolgenden Umsetzung geschützt werden, da aufgrund des aciden Protons am Pyrazol unerwünschte, polymere Nebenprodukte entstehen können. Eine geeignete Schutzgruppe stellt die THPGruppe dar. Die Einführung dieser erfolgt durch Umsetzung mit 2,3-Dihydropyran (siehe Schema 2.1).

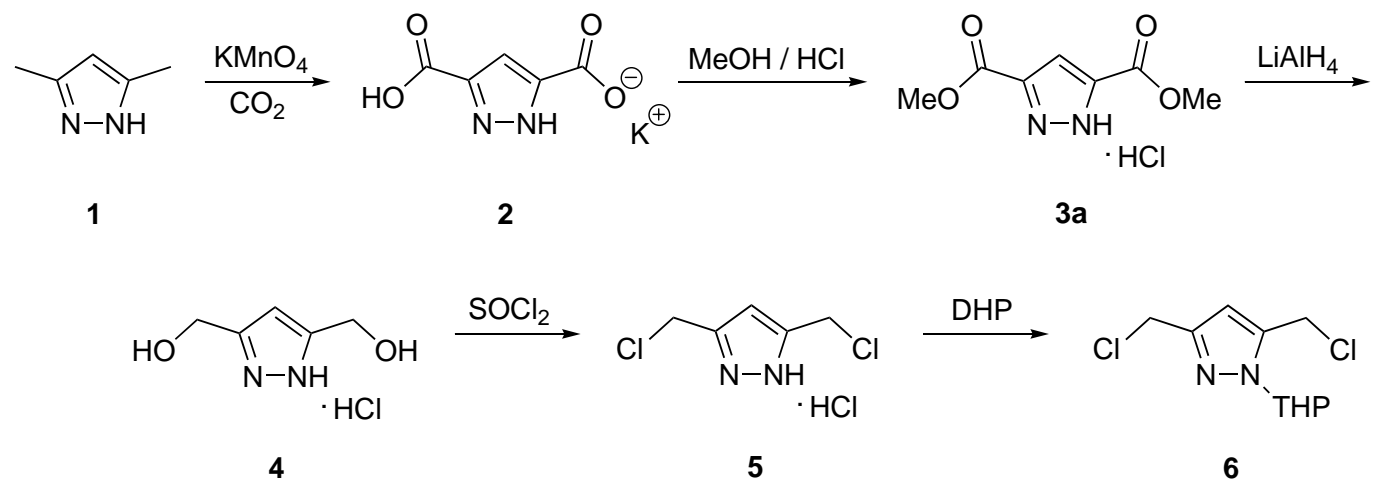

Schema 2.1: Darstellung von 3,5-Bis(chlormethyl)-1-(tetrahydropyran-2-yl)-1H-pyrazol (6).

Durch die Einführung einer Phenylgruppe im Rückgrat des Pyrazols ist es möglich die Liganden zu modifizieren. Somit ist eine Verbesserung bezüglich der Löslichkeit sowie Kristallisationseigenschaften zu erwarten. 3,5-Bis(chlormethyl)-1(tetrahydropyran-2-yl)-4-phenyl-1H-pyrazol (12) konnte in einer sechsstufigen Synthese dargestellt werden [40-43]: Das kommerziell erhältliche Glycinmethylester Hydrochlorid (7) wird mit Natriumnitrit unter sauren Bedingungen zu Diazoessigsäuremethylester (8) umgesetzt. Der Ringschluss erfolgt durch Reaktion von 8 mit Zimtsäuremethylester zum Pyrazolin (9), welches mit Brom zu 4-Phenyl-1Hpyrazol-3,5-dicarbonsäuremethylester (3b) oxidiert wird. Analog zu der Verbindung 3a wird der Ester $\mathbf{3 b}$ mit LAH reduziert, mit Thionylchlorid chloriert und anschlieBend mittels der THP-Gruppe geschützt. 3,5-Bis(chlormethyl)-1-(tetrahydropyran2-yl)-4-phenyl-1 $H$-pyrazol (12) konnte in guter Ausbeute isoliert werden (siehe Schema 2.2).

\subsubsection{N-substituierte Imidazole: Die Seitenarme}

Die Synthese der $N$-substituierten Imidazole kann auf mehreren Wegen erfolgen [4446] und wurde erstmalig von Arduengo patentiert. [47] Eine Möglichkeit, welche bei 
<smiles>COC(=O)C=Cc1ccccc1</smiles><smiles>COC(=O)c1n[nH]c(C(=O)OC)c1-c1ccccc1</smiles>

3b

10<smiles>ClCc1n[nH]c(CCl)c1-c1ccccc1</smiles>

Schema 2.2: Synthese von 3,5-Bis(chlormethyl)-1-(tetrahydropyran-2-yl)-4-phenyl-1Hpyrazol (12).

dieser Arbeit verfolgt wurde, besteht darin, äquimolare Mengen von Formaldehyd, Glyoxal und einem primären Amin umzusetzen (Schema 2.3) und findet auf Grund ihrer Einfachheit verbreitet Anwendung. Bei dieser Reaktion handelt es sich um eine einstufige Ringschlusssynthese, welche unter sauren Bedingungen durchgeführt werden sollte. Dadurch werden mögliche Nebenreaktionen zwischen Glyoxal und Amin (z.B. eine Cannizzaro-Reaktion) vermieden, welche für die in der Literatur beschriebenen schlechten bis mittelmäßigen Ausbeuten verantwortlich sein können. Diese Syntheseroute wird meistens für 1-Alkylimidazole angewendet. Die Reaktionsbedingungen wurden im Laufe der Jahre weiter optimiert [48, 49]: Eckdaten wie Reaktionsdauer und Verhältnis der beteiligten Edukte zueinander wurden so gewählt, dass einfach und aus leicht zugänglichen Edukten Ansätze mit größeren Mengen durchgeführt werden konnten. Zusätzlich konnten während der Aufarbeitung durch pH-Wert-Erhöhung in den stark basischen Bereich mittels Zugabe einer Natriumhydrogencarbonat-Lösung in Kombination mit Natrium- bzw. Kaliumhydroxid (als Feststoff), bessere Ausbeuten erzielt werden. Hierbei ist darauf zu achten, dass, wenn die Reaktionslösung ausschließlich und direkt in einer Natriumbzw. Kaliumhydroxid-Lösung gegeben wird, die Ausbeute bedeutend sinkt und die Aufarbeitung erschwert wird. 


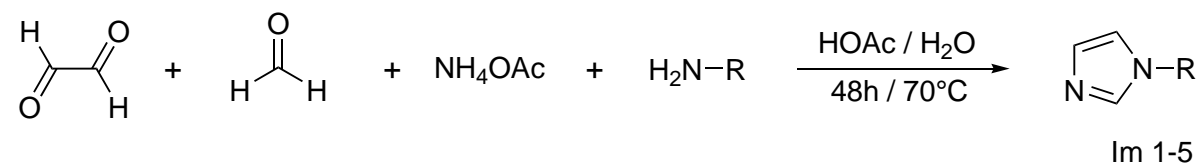

$$
\begin{aligned}
& \text { Im } 1(R=t B u) \\
& \text { Im } 2(R=1-\text { Adamantyl }) \\
& \text { Im } 3(R=2,4,6-\text { Trimethylphenyl }) \\
& \text { Im } 4(R=1-B e n z y l) \\
& \text { Im } 5(R=(S)-1 \text {-Phenylethyl })
\end{aligned}
$$

Schema 2.3: Gängige Syntheseroute der $N$-substituierten Imidazole.

Imidazole, welche aliphatische Reste tragen, konnten nicht mit guten Ausbeuten über die oben erwähnte 1-Aryl Substitution synthetisiert werden. Das 1-(1Adamantyl)- $1 H$-imidazol beispielsweise kann in besseren Ausbeuten durch die direkte Umsetzung von $1 H$-Imidazol mit 1-Adamantylbromid in Form einer Schmelze dargestellt werden. [50-52] Das reine Produkt lässt sich nach säulenchromatographischer Aufarbeitung, oder alternativ durch Sublimation als weißer Feststoff gewinnen. Das 4-(1-Adamantyl)imidazol, das bei der Reaktion als Nebenprodukt entsteht, kann somit abgetrennt werden (Schema 2.4).

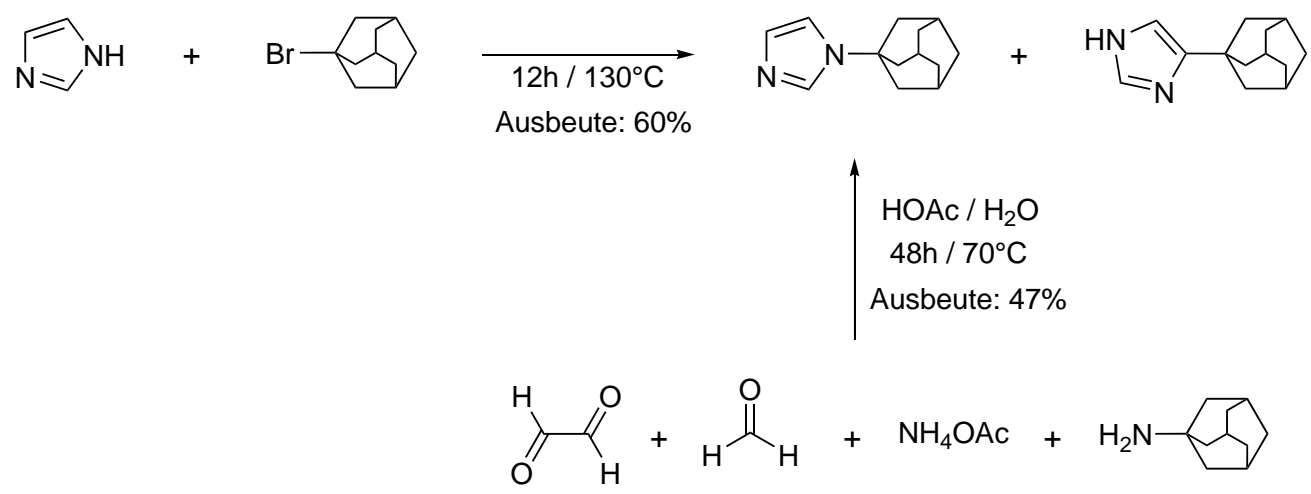

Schema 2.4: Alternative Synthese von 1-(1-Adamantyl)-1H-imidazol im Vergleich zur 1-Aryl Substitution. Die Abtrennung des Nebenproduktes erfolgt durch Säulenchromatographie oder Sublimation.

Eine modifizierte Synthese in Anlehnung zu Literaturstelle [44] wurde für das 1(tert-Butyl)-1H-imidazol durchgeführt (Schema 2.5). Hierbei lässt sich sowohl eine aufwändige Aufarbeitung als auch ein Reinigungsschritt mittels Destillation nicht vermeiden.

Bei der Vielzahl der substituierten Imidazole, welche für diese Arbeit synthetisiert wurden, reiht sich eins mit aliphatischen, chiralen Rest ein. Das 1-(1-Phenylethyl)- 


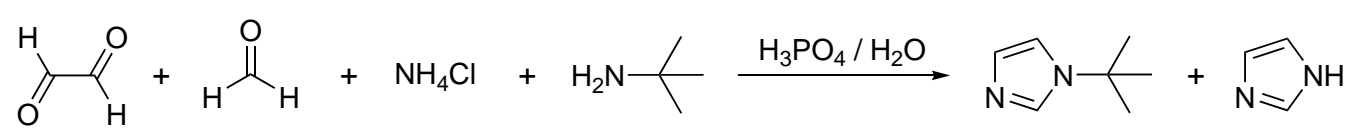

Schema 2.5: Synthese von 1-(tert-Butyl)-1H-imidazol.

$1 H$-imidazol war problemlos in guter Ausbeute über die 1-Aryl Substitutionsroute zugänglich.

Teilweise konnten auch kommerziell erhältliche Imidazolderivate eingesetzt werden, wie beispielsweise das 1-Benzyl-1 $H$-imidazol, sowie das 1-(4-Nitrophenyl)-1Himidazol. Letzteres kann alternativ aus preiswerten Ausgangsverbindungen durch direkte Reaktion von $1 H$-Imidazol mit 1-Fluor-4-nitrobenzol, Natriumcarbonat und $N, N$-Dimethylformamid synthetisiert werden. [53, 54]

\subsection{Synthesemöglichkeiten von NHCs}

Lange Zeit vor der Isolierung von freien Carbenen haben Öfele [15] und Wanzlick [16] über die ersten Komplexe mit NHC-Liganden berichtet (siehe Kapitel 1.3, Schema 1.5). Inzwischen wurden NHC-Metallkomplexe mit einer Vielzahl von Hauptgruppen- und Übergangsmetallen dargestellt. [55]

Für die Synthese dieser sind mehrere Wege denkbar. [56] Die wichtigsten werden hier kurz erläutert: Eine Möglichkeit ist die in situ Deprotonierung eines Ligandvorläufers - meist von Azoliumsalzen. Dafür sorgt der Zusatz eines basischen Metallsalzes, bspw. eines Acetats oder Alkoxides (Schema 2.6). Alternativ können Brønstedtbasische Metallate, meist unter Erwärmung (Schema 2.7) oder auch externe Basen (Schema 2.8i)) verwendet werden. Die externe Base muss sorgfältig gewählt werden, da unter Umständen ungewünschte Nebenreaktionen auftreten können. Basen wie Kalium-tert-butanolat und Kalium-bis-(trimethylsilyl)amid sind gut einsetzbar. Für die Deprotonierung sind hydridübertragende Basen wie Lithiumaluminiumhydrid eher ungeeignet, da diese zu einem Imidazolidin XII anstelle des erwünschten Carbens XI führen können (Schema 2.8ii)). [57] Sogar das $\eta^{5}$-CyclopentadienylAnion kann ebenso gut als Deprotonierungs-Reagenz fungieren (Schema 2.9). [58] Diese Methode ist dann sinnvoll anzuwenden, wenn das beteiligte freie Carben als instabil oder nicht einfach in der Handhabung eingestuft wird.

Ferner besteht die Möglichkeit, NHC-Komplexe über Ligandentransfer-Reaktionen zu erhalten. Wang und Lin entwickelten eine Methode, die die Synthese von NHC- 

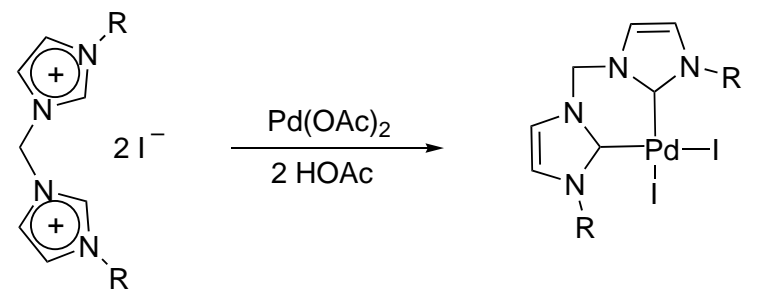

Schema 2.6: In situ Deprotonierung eines NHC-Liganden unter Verwendung von $\mathrm{Pd}(\mathrm{OAc})_{2}$.<smiles></smiles>

Schema 2.7: In situ Deprotonierung am Beispiel eines Cr-NHC-Komplexes nach Öfele. Das basische Metallion $\left[\mathrm{HCr}(\mathrm{CO})_{5}\right]^{-}$dient gleichzeitig als Base und Ligandakzeptor.

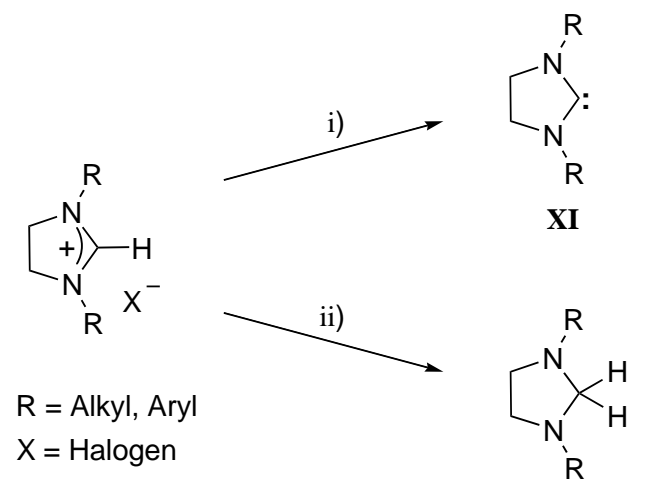

Schema 2.8: Deprotonierung eines NHC-Liganden i) unter Verwendung der externen Base $\mathrm{KO}^{t} \mathrm{Bu}$ und ii) mit $\mathrm{LiAlH}_{4}$; mögliche unerwünschte Nebenreaktion zum Imidazolidin XII.
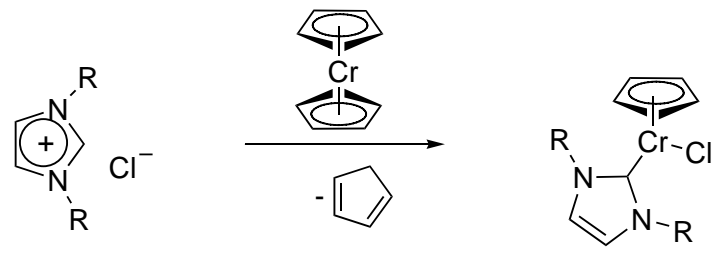

Schema 2.9: Deprotonierungsreaktion eines NHC mittels $\eta^{5}$-Cyclopentadienyl-Anion. 
Metallkomplexen über deren Silber(I)-Carbenkomplexe ermöglicht. [59] Vorteile dieser Synthese liegen in der Einfachheit der Durchführung, der Aufarbeitung bzw. Aufreinigung; das Arbeiten unter Luft- und Feuchtigkeitsausschluss ist nicht erforderlich. Diese Synthesemethode findet verbreitet Anwendung und stellt somit eine gute Alternative zur in situ Deprotonierung dar.

Die einfachste Methode ist allerdings die direkte Umsetzung freier Carbene mit einem geeigneten Metallvorläuferkomplex. Das freie Carben ist in der Lage, dimere Komplexe mit verbrückenden Einheiten wie $\mu$-CO oder $\mu$-Cl aufzubrechen sowie schwach koordinierende Liganden wie CO, COD oder Phosphan leicht auszutauschen (Schema 2.10). Diese Synthesemethode ist aufwändig und bedarf der Isolation des freien Carbens, das umgesetzt wird.

2<smiles></smiles>

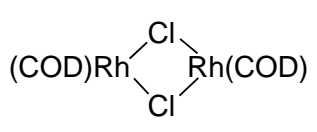<smiles>[R]N1C=CN([R])C1C1C2CCC(C2)[R7]1(Cl)CCC</smiles>

Schema 2.10: Umsetzung eines freien Carbens mit einem Metallvorläuferkomplex.

Eine weitere Methode $N$-heterozyklische Carbenkomplexe zu synthetisieren bietet die Spaltung elektronenreicher Olefine. [60] Der entsprechende Komplex kann leicht aus einer thermischen Spaltung von Tetraaminoethylen-Derivaten in Gegenwart von Metallvorläufern (z.B. Carbonylkomplexen) erhalten werden. Schema 2.11 zeigt eine solche Spaltung. Mit Hilfe dieser Methode gelang es Lappert et al. eine ganze Reihe von NHC-Übergangsmetallkomplexen herzustellen. [18, 60, 61]<smiles>[R]N1CCN([R])C1=C1N([R])CCN1[R]</smiles>

Schema 2.11: Darstellung eines Fe-NHC-Komplexes durch thermische Spaltung des entsprechenden Tetraaminoethylens mit Eisenpentacarbonyl.

\subsubsection{Im Fokus dieser Arbeit: Silber-NHC-Komplexe}

Von besonderem Interesse sind in der Carben-Chemie die Silber(I)-NHC-Komplexe, da diese sich zum einen als Transmetallierungs-Reagenzien eignen und zum anderen ungewöhnliche strukturelle und optische Eigenschaften vorweisen. 
Der erste Silber-NHC-Komplex geht auf Arduengo im Jahr 1993 zurück. [62] Schema 2.12 zeigt die von ihm gefundene Umsetzung.

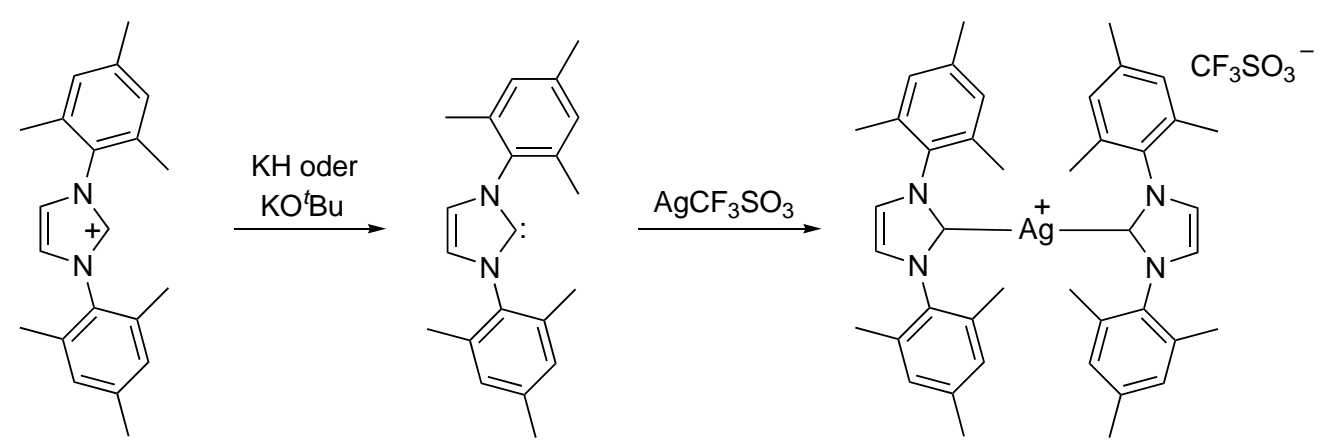

Schema 2.12: Synthese des ersten Silber-NHC-Komplexes nach Arduengo.

Seither ist eine Vielzahl von Silber-NHC-Komplexen hergestellt worden. Die geeignetste und weitverbreitete Methode, um zu NHC-Komplexen zu gelangen, stellt die Transmetallierung von Silber(I)-NHC-Komplexen dar. Diese sind in der Regel einfach über die sogenannte " $\mathrm{Ag}_{2} \mathrm{O}$-Syntheseroute“ zugänglich. Der große Vorteil dieser Syntheseroute besteht darin, dass nur der entsprechende Ligandvorläufer (in dieser Arbeit: das Imidazoliumsalz) und $\mathrm{Ag}_{2} \mathrm{O}$ erforderlich sind. Das Silber(I)oxid fungiert hierbei sowohl als milde Base, welches für die in situ Deprotonierung des Ligandvorläufers (bzw. des Imidazoliumsalzes) sorgt, als auch als das anschließend im Komplex eingebaute Metallion. [63] Die Durchführung verläuft unter milden Bedingungen (meist bei RT) mit Ausnahme von Imidazoliumsalzen mit sterisch anspruchsvollen Substituenten, welche erst unter Rückfluss vom Silber-Salz deprotoniert werden können. Die Reaktionen sind in diversen Lösungsmitteln durchführbar, in unpolaren genauso gut wie in polaren, darunter auch in Wasser. Bei dieser Syntheseroute kann man meist Arbeiten unter Luft- und Feuchtigkeitsausschluss völlig vermeiden. Die resultierenden Silber(I)-NHC-Komplexe sind luftstabil, zeigen aber in gelöster Form häufig eine hohe Lichtempfindlichkeit.

Alternativ können auch andere Silber-Basen verwendet werden: Bertrand et al. synthetisierten den ersten Silber-NHC-Komplex in polymerer Form ausgehend vom jeweiligen Imidazoliumsalz und AgOAc [64, 65] und kurze Zeit später berichteten Danopoulos und Mitarbeiter über die Darstellung einer Reihe von NHC-Komplexen unter Verwendung von $\mathrm{Ag}_{2} \mathrm{CO}_{3}$. [66] Diese Silber-Basen sind allerdings wegen des großen Erfolges der „ $\mathrm{Ag}_{2} \mathrm{O}$-Route“ größtenteils in den Hintergrund gerückt.

Mehrere Publikationen sind in den letzten Jahren erschienen, in denen die Anwendung dieser Synthesemethode beschrieben wird. Da die Komplexe leicht zugänglich 
und synthetisch wertvoll sind, sind einige Silber(I)-NHC-Komplexe untersucht worden. Mehrere Übersichtsartikel sind verfügbar. [67-69]

Es können vielfältige strukturelle Motive der Silber-NHC-Komplexe unter Variation der Reaktionsbedingungen, der verwendeten Anionen sowie durch verschiedene sterische und elektronische Eigenschaften der NHC-Liganden realisiert werden. Für Silber-NHC-Komplexe mit koordinierenden Anionen, wie beispielsweise Halogenide, wurden diverse Bindungsmotive in der Festkörperstruktur gefunden (Abbildung 2.1). [67] Hierbei wurde zusätzlich eine Unterteilung in Einfach-NHC(mit nur einem NHC pro Ligand) und Mehrfach-NHC-Komplexe (mit mehreren NHCs pro Ligand) vorgenommen. Gezeigt ist dies am Beispiel des KoordinationsTyps (C-Ag-X) (Abbildung 2.1; a1 und a2). Somit gibt es einfach koordinierende $(\mathrm{C}-\mathrm{Ag}-\mathrm{X})$, verbrückende $\left(\mathrm{C}-\mathrm{Ag}-\mathrm{X}_{2}\right)$ und treppenartige $\left(\mathrm{C}-\mathrm{Ag}-\mathrm{X}_{3}\right)$ Strukturen. Möglich sind auch Strukturen mit einem Halogen-Gegenion des Typs $\left[\mathrm{AgX}_{2}\right]^{-}$ $\left(\mathrm{C}_{2}-\mathrm{Ag}-\mathrm{AgX} \mathrm{X}_{2}\right)$ oder mit einem Halogen-Gegenion wie $\left[\mathrm{Ag}_{2} \mathrm{X}_{4}\right]^{2-}$ mit $\mathrm{Ag}-\mathrm{Ag}$ Wechselwirkung, welche über drei Donorgruppen stabilisiert werden. Im Fall des Koordinations-Typs $(\mathrm{C}-\mathrm{Ag}-\mathrm{X})$ sind die Komplexe neutral und besitzen eine lineare Koordinations-Geometrie, sowohl mit der NHC-Einheit als auch mit dem Anion, das meist ein Halogenid ist (Abbildung 2.1; a1 und a2). Im KoordinationsTyp $\mathrm{C}-\mathrm{Ag}-\mathrm{X}_{2}$ besteht eine Verbrückung der (zwei) Silber-Atomen über Halogenide. Jedes Silber-Atom ist außerdem mit je einer Carben-Einheit verbunden. Neben Silber-NHC-Komplexen mit koordinierenden Anionen sind eine Reihe weiterer Silber-NHC-Komplexe mit nichtkoordinierenden Anionen in der Literatur beschrieben worden. [67] Der Hauptvertreter ist vom Typ $\left(\mathrm{C}_{2}-\mathrm{Ag}\right)$ und ist in Abbildung $2.1 \mathrm{f}$ gezeigt. Dieser besteht als eine Bis-Carben-Verbindung in der Festkörperstruktur. Jedes Silber-Kation ist von zwei NHCs koordiniert. Je nach sterischem Anspruch der verwendeten Substituenten können die NHC-Ringe rotieren, um eine energetisch günstige Position im Kristall einzunehmen.

Die Silber-NHC-Komplexe können insofern als mehrkernige Aggregate vorliegen. In der Literatur ist bereits über linear angeordnete di- [70, 71] und trinukleare [72] Ag-Strukturen, sowie tri-, [73-75] tera-, [34, 76, 77] hexa- [78] und octanukleare [79] Ag-Cluster berichtet worden.

Das strukturelle Motiv, welches bei Münzmetallen in Verbindung mit Pyrazolatkomplexen üblicherweise auftritt, ist ein planares Trimer $\left[\mathrm{M}^{\mathrm{I}}\left(\mu-\mathrm{Pz}^{*}\right)\right]_{3}\left(\mathrm{Pz}^{*}\right.$ gibt das unterschiedlich substituierte Pyrazol an) (Abbildung 2.2; Typ A). [80-83] Dieses Strukturmotiv ist dann am wahrscheinlichsten, wenn der sterische Anspruch der am Pyrazolat gebundenen Substituenten groß ist. Im Gegensatz zu diesem trinuklearen Motiv sind tetranukleare und hexanukleare Strukturen seltener beobachtet 
worden. Im Fall von Silber(I)-Komplexen mit Pyrazolat-Einheiten sind eindimensionale Polymere $[\mathrm{Ag}(\mu-\mathrm{Pz})]_{\mathrm{n}^{-}}$und diverse unterschiedlich substituierte Trimere $\left[\mathrm{Ag}\left(\mu-\mathrm{Pz}^{*}\right)\right]_{3}$-Strukturen beschrieben worden. [84]<smiles>[R]N1C=CN([R])C1[Al][V]</smiles>

a) C-Ag-X $X=$ Halogen<smiles>[R]N1C=CN([R])C1[Ga]([Y])[Al]</smiles>

b) C-Ag- $\mathrm{X}_{2}$

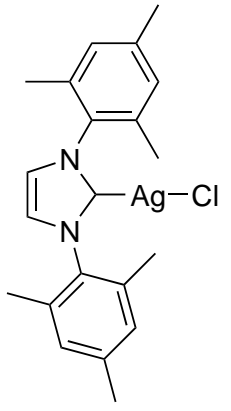

a1) Einfach-NHC C-Ag-X

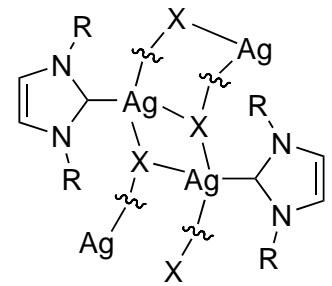

c) C-Ag- $X_{3}$<smiles>CN1C=CN(Cc2cccc(CN3C=CN(C)C3[Ge]Cl)c2)C1[Ge]Cl</smiles>

a2) Mehrfach-NHC C-Ag-X<smiles></smiles>

d) $\mathrm{C}_{2}-\mathrm{Ag}-\mathrm{Ag} \mathrm{X}_{2}$<smiles>[Y]N1C=CN([R])C1[Se]C1N([R])C=CN1[R]</smiles>

f) $\mathrm{C}_{2}-\mathrm{Ag}$

Abbildung 2.1: Koordinationsmöglichkeiten von Silber-NHC-Komplexen bisher.

Ein tetramerer Silber(I)-Komplex wurde im Jahr 2007 von Raptis und Mitarbeitern strukturell charakterisiert. [84] Obwohl die Substituenten am Pyrazol mit $\mathrm{R}=t \mathrm{Bu}$ räumlich anspruchsvoll sind, konnte eine sattelförmige tetramere Struktur vom Typ B isoliert werden.

Weiterhin wird über pyrazolverbrückte Bis(NHC)Liganden berichtet. [34-37] Diese Liganden erlauben die Synthese von mehrkernigen Silber(I)-NHC-Komplexen. Es wurde beobachtet, dass Verbindungen mit der allgemeinen Formel $\left[\mathrm{LAg}_{2}\right]_{\mathrm{n}}$ zwei sehr unterschiedliche Strukturen besitzen (Abbildung 2.3): Ein planarer $\mathrm{Ag}_{4} \mathrm{Kern}$ 


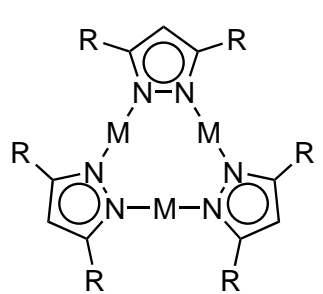

A

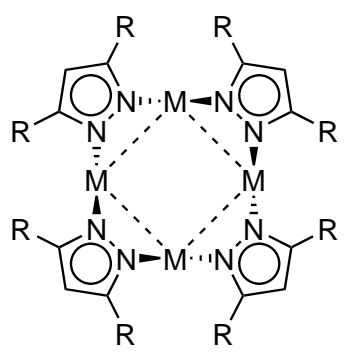

B

Abbildung 2.2: Mögliche strukturelle Motive A und B von Münzmetall-PyrazolatKomplexen. [79]

eingeschlossen zwischen zwei Pyrazolat-NHC Liganden $(\mathbf{C} ; \mathbf{n}=2)$ oder eine neuartige $\mathrm{Ag}_{8}$ Doppelmetallkrone $(\mathbf{D} ; \mathrm{n}=4)$.

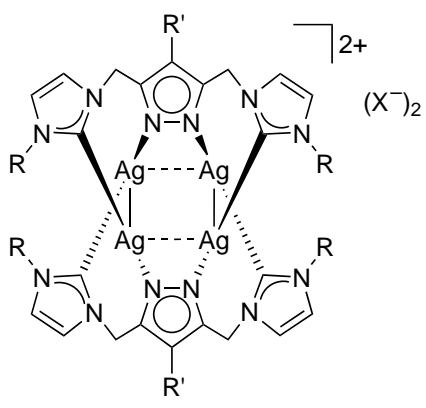

C

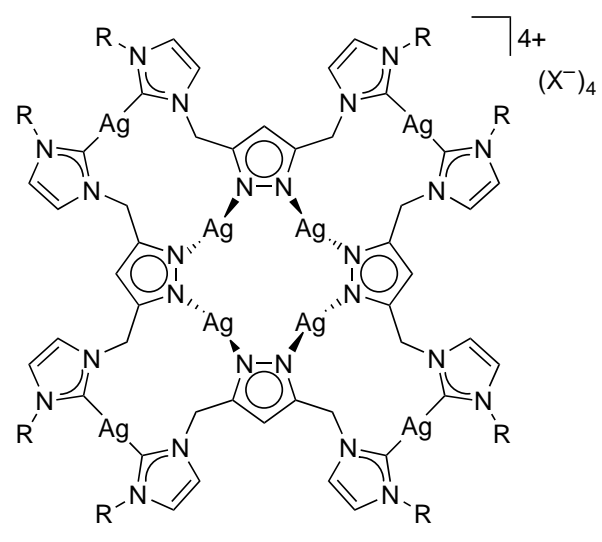

D

Abbildung 2.3: Unterschiedliche Strukturen von mehrkernigen pyrazolverbrückten Silber(I)-NHC-Komplexen $\mathbf{C}$ und $\mathbf{D}$.

Komplexe des Typs D unterscheiden sich maßgeblich von den bisher strukturell identifizierten Silber-Komplexen. Dieser achtkernige Komplex weist eine doppelkronenartige Struktur auf: die innere Krone mit einer homoleptischen $\mathrm{N}$ (Pyrazolat)-Koordination und die umliegende, äußere Krone mit einer homoleptischen Bis(NHC)-Koordination. Die vier inneren, zentralen Silber(I)ionen definieren eine beinahe quadratische Ebene, welche durch die Pyrazolat-Einheiten verbrückt ist. Diese sind abwechselnd ober- und unterhalb dieser Ebene angeordnet. Diese Struktur kombiniert ebenso zwei der gängigsten strukturellen Koordinationsmöglichkeiten in der Silber(I)-Koordinationschemie, nämlich die lineare $\left[\mathrm{Ag}(\mathrm{NHC})_{2}\right]^{+}$ und die zyklische $[\mathrm{M}(\mu-\mathrm{Pz})]_{\mathrm{n}}$-Anordnung (meistens mit $n=3$; siehe Typ A). Diese Tatsache weckt interessante Fragen über die Beziehung und mögliche Konkurrenz 
zwischen diesen beiden Bindungsmotiven. Es wurde spekuliert, dass die Bildung von unterschiedlichen Strukturtypen $\mathbf{C}$ oder D trotz ähnlichem Liganden-Gerüst und identischem Ligand-zu-Metall Verhältnis von 2:1 bzw. 4:2 in den Silber(I)Pyrazolat-NHC-Systemen, durch den unterschiedlichen sterischen Anspruch der NHC-Substituenten verursacht werden könnte.

Hinzu konnte im Rahmen dieser Arbeit ein neuartiger Silber-NHC-Komplex mit einem Ligand-zu-Metall Verhältnis von 4:4 isoliert und strukturell charakterisiert werden (siehe Kapitel 4.2.1).

Neben ihrer Anwendung als Ligandtransfer-Reagenzien bieten Silber(I)-NHCKomplexe weitere Anwendungsmöglichkeiten von besonderem Interesse, z.B. als Lumineszenzmaterialien, da sie oft ungewöhnliche optische Eigenschaften besitzen, oder auch als Vorläufer für Ag-Nanopartikel.

Die Lumineszenz-Eigenschaften von Münzmetall-Komplexen wurden intensiv untersucht, während die von den analogen NHC-Komplexen weniger Aufmerksamkeit bekommen haben. Es gibt zahlreiche Veröffentlichungen zu Systemen, in denen mehrkernige $\mathrm{Au}(\mathrm{I})$-Komplexe diese Eigenschaften zeigen. Hierfür verantwortlich ist die sogenannte aurophile Wechselwirkung [85] zwischen den $\mathrm{d}^{10} \mathrm{Au}(\mathrm{I})$-Zentren. Im Gegensatz dazu wurde Lumineszenz in Cu-NHC-Komplexen nicht beobachtet. [69] Lumineszenz wird allerdings in vielen multinuklearen $\mathrm{Ag}(\mathrm{I})-\mathrm{NHC}-K o m p l e x e n$ beschrieben. [34, 72, 86] Bei einigen dieser Ag(I)-NHC-Komplexe wurde der Austausch der $\mathrm{Ag}(\mathrm{I})$-Atome vorgenommen, welcher $\mathrm{zu}$ isostrukturellen $\mathrm{Au}(\mathrm{I})-\mathrm{NHC}$ Komplexen führte. [34, 87] Die Lumineszenz-Eigenschaften der $\mathrm{Ag}(\mathrm{I})$ - und $\mathrm{Au}(\mathrm{I})$ NHC-Komplexe beruhen typischerweise auch auf Übergängen, die durch die Art des Liganden stark beeinflusst werden. Heterometallische $\mathrm{Au}(\mathrm{I})-\mathrm{Ag}(\mathrm{I})-\mathrm{Komplexe}$, die NHCs beinhalten und die Fähigkeit zur Lumineszenz aufweisen, sind weniger untersucht. Erste Ergebnisse wurden im Jahr 2005 von Catalano et al. berichtet. [74] Aufgrund der unterschiedlichen Metalle werden die Wechselwirkungen zwischen diesen verstärkt. Dies führt zu kürzeren Metall-Metall Bindungsabständen als in den entsprechenden homometallischen Komplexen.

\subsection{Anwendungsmöglichkeit: Katalyse}

Die Einführung eines Katalysators in eine chemische Reaktion eröffnet neue Reaktionswege mit niedrigerer Aktivierungsenergie, indem dieser aktive Zwischenstufen bildet oder mehrere Oxidationsstufen durchläuft. [88] Es ist wichtig hierbei anzumerken, dass die thermodynamische Gleichgewichtslage bei der Reaktion durch 
Verwendung von Katalysatoren nicht verändert werden kann. Der Katalysator beeinflusst nur die Geschwindigkeit oder den Verlauf einer Reaktion und geht selbst unverändert aus der Reaktion hervor.

Katalytische Reaktionen waren seit langer Zeit bekannt, ohne dass sie als solche erkannt wurden. Die ersten katalytischen Prozesse wurden durch Enzyme (Biokatalysatoren) katalysiert. Der Einsatz von Katalysatoren ist für die chemische Industrie, aber auch in vielen Umweltschutztechniken heutzutage nicht mehr wegzudenken. Produkte, die aus chemischen Verbindungen hergestellt werden, welche erst durch katalytische Verfahren wirtschaftlich produziert werden können, sind im alltäglichen Leben unverzichtbar geworden. Etwa $75 \%$ aller Produkte der chemischen Industrie werden durch Verwendung von Katalysatoren hergestellt, bei den neu entwickelten Verfahren sind es sogar 90\%. [88]

Je nach ihrer Wirkungsweise unterscheidet man zwischen heterogenen und homogenen Katalysatoren. Erstere sind meist Feststoffe mit einer sehr großen Oberfläche, sodass während der entsprechenden Reaktion mehrere Phasen vorliegen, und letztere wirken molekulardispers im Reaktionsmedium. Als Katalysatoren werden in der Regel Metallkomplexe eingesetzt, die sich üblicherweise aus einem frühen oder späten Übergangsmetall als Zentralatom und einem komplexierenden Liganden zusammensetzen. [89] Die Auswahl des Liganden muss sorgfältig geschehen und beeinflusst die Selektivität und Aktivität in einer katalytischen Reaktion erheblich. Indem man die Liganden geeignet im Bezug auf ihren sterischen und elektronischen Eigenschaften modifiziert, können diese für jede katalytische Reaktion gezielt eingestellt werden. Häufig werden Phosphorverbindungen wie Phosphane eingesetzt.

Durch die Einführung von Chiralität in die Ligandsysteme ist es möglich, asymmetrische Katalyse durchzuführen. Der chirale Ligand hat die Aufgabe das Metall zu stabilisieren sowie eine für die Katalyse geeignete Komplexgeometrie einzustellen. Das Molekül wird dabei so orientiert, dass ein anderes Substratmolekül selektiv nur von einer vorbestimmten Seite aus angreifen kann. Dazu wird durch das geeignete Ligandsystem die Elektronendichte am Metall eingestellt, das außerdem im Laufe des Katalysezyklus verschiedene Oxidationstufen durchlaufen kann. Kommt es bei der Katalyse zum Einsatz von bimetallischen Komplexen, ist eine kooperative Reaktivität zu erwarten. Solche kooperativen Effekte sollen auch bei der Zwei-Zentren Katalyse erzielt werden. [90] Diese Herangehensweise wird oft in der Natur bei enzymatischen Prozessen gefunden. Die asymmetrische Katalyse gewinnt immer mehr an Bedeutung, da sie z.B. für die Herstellung von Arzneimitteln unverzichtbar ist. Im Jahre 2001 wurden Noyori, Sharpless und Knowles für ihre bahnbrechenden Arbeiten auf diesem Gebiet, welche in vielen industriellen Verfahren von Nutzen sein werden, mit dem Nobelpreis ausgezeichnet. [91] 


\subsubsection{NHC-Komplexe in der Katalyse}

Eine weitere - und die mit Abstand nennenswerteste Anwendung - findet die Substanzklasse der NHC-Komplexe in der metallorganischen Katalyse, wo sie allmählich die Phosphane verdrängen. Diese werden häufig für ähnliche bzw. identische katalytische Prozesse verwendet, z.B. bei den palladiumvermittelten Kupplungsreaktionen. [55, 92] Die vorteilhaften Eigenschaften der NHCs, mitunter der ausgeprägte $\sigma$-Donorcharakter, die Einstellung der sterischen Umgebung und die starke Metall-NHC-Bindung(en) (siehe Kapitel 1.4), haben zu zahlreichen intensiven Untersuchungen der Katalyseeigenschaften geführt und ermöglichen so den Einsatz von Metall-NHC-Komplexen in einer großen Anzahl an C-C Kupplungsreaktionen. Sie finden Anwendung z.B. bei der Heck- [20, 93], Suzuki- [94], Sonogashira- [95, 96], Stille- [97] und Kumada- [98] Kupplung. Zusätzlich spielen sie eine Rolle in der Hydrierung [99, 100], Hydroformylierung [101] und Hydrosilylierung [102, 103]. Vor allem Metathesereaktionen haben aufgrund ihrer Reaktionsvielfalt (Ringschluss-, Ringöffnungs-, Kreuzmetathese und Polymerisationen) an Bedeutung gewonnen. [104]

Der heutzutage wichtigste industrielle Katalyseprozess unter Verwendung von NHCKomplexen ist die Olefinmetathese. Hierbei findet die Umalkylidenierung zweier Doppelbindungen unter Beteiligung eines Katalysators, der Alkylidenkomplexe mit unterschiedlichen Liganden, statt. Bis heute zählen Ruthenium-NHC-Komplexe zu den aktivsten Katalysatoren in der Olefinmetathese. [105-107] Zudem haben diese die höchste Effizienz von allen bisher bekannten Systemen bewiesen. Ein von Grubbs entwickelter Katalysator ist in Abbildung 2.4 (XIII) dargestellt. [108] Der Austausch eines Phosphan- durch einen NHC-Liganden ermöglicht den Zugang zu den sogenannten Grubbs-Katalysator(en) der zweiten Generation (Abbildung 2.4 (XIV)). Durch diese Modifizierung ist eine viel aktivere Spezies (XIV) entstanden, die eine merklich bessere Luft- sowie Feuchtigkeitsstabilität besitzt. Des Weiteren lassen sich die Komplexeigenschaften des Produktes gezielt einstellen, indem die NHC-Liganden am Katalysator variiert werden. Grubbs und Schrock wurden gemeinsam mit Chauvin im Jahr 2005 für ihre bedeutende Forschung auf dem Gebiet der reaktiven Metall-Carben-Komplexe und deren Anwendung in der Olefinmetathese mit dem Nobelpreis für Chemie geehrt. [109-111]

Es gibt also viele katalytische Systeme, welche NHC-Liganden beinhalten. Zudem hat sich im Laufe der Jahre das Pyrazol als eine geeignete verbrückende Einheit erwiesen. [34, 35] NHC-Bausteine in Kombination mit Pyrazol eignen sich sehr gut als Liganden und lassen sich an mehreren Übergangsmetallen koordinieren, wobei sie auch bimetallische Systeme bilden können. 


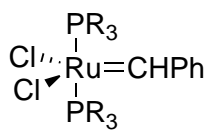

XIII

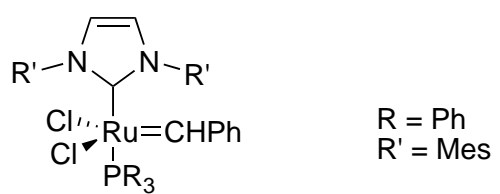

XIV

Abbildung 2.4: Grubbs-Metathesekatalysatoren der ersten (XIII) und zweiten (XIV) Generation.

Für diese bimetallischen NHC-Komplexe ist eine kooperative MehrzentrenReaktivität zu erwarten. Aus natürlichen Vorbildern sind katalytische Reaktionen mit kooperativer Mehrzentren-Reaktivität bekannt und weit verbreitet. Solche kooperativen Effekte können auch in der Zwei-Zentren Katalyse von Nutzen sein. Kürzlich durchgeführte Forschungsarbeiten zeigen ein ansteigendes Interesse auf diesem Gebiet. [90]

$\mathrm{Zu}$ den C-C Kupplungsreaktionen in der organischen Synthese gehören auch allylische Substitutionsreaktionen. Ein Vertreter ist die Palladium-katalysierte Alkylierung. Bei Verwendung chiraler Liganden am Palladium besteht ebenfalls die Möglichkeit asymmetrische Katalyse durchzuführen. Somit ist die Darstellung enantiomerenreiner Produkte möglich.

Der Mechanismus der Palladium-katalysierten allylischen Substitution war Gegenstand verschiedener Untersuchungen und gilt heute als aufgeklärt. [38, 112-114] Er wird durch folgenden Katalysezyklus beschrieben (Schema 2.13): Als Ausgangspunkt dient hierbei eine direkt eingesetzte oder in situ erzeugte $\operatorname{Pd}(0)$-Spezies. Aus dieser entsteht durch Koordination des Substratmoleküls unter Abspaltung der Abgangsgruppe ein Olefin-Palladium-Komplex, welcher in einer oxidativen Addition in einen Palladium- $\eta^{3}$-Komplex überführt wird. Darauffolgend wird dieser von einem Nukleophil von der gegenüberliegenden Seite zum Metall im geschwindigkeitsbestimmenden Schritt angegriffen. [115] Der Produkt-Olefin-Komplex setzt anschließend das Produkt unter Regeneration der $\operatorname{Pd}(0)$-Spezies frei.

Gezeigt ist das am Beispiel der allylischen Substitution von Dimethylmalonat mit 1,3-Diphenylallylacetat (Schema 2.14). Diese Reaktion gilt als Maßstab zur Überprüfung der katalytischen Eignung und Aktivität solcher Systeme bei der allylischen Substitution. 


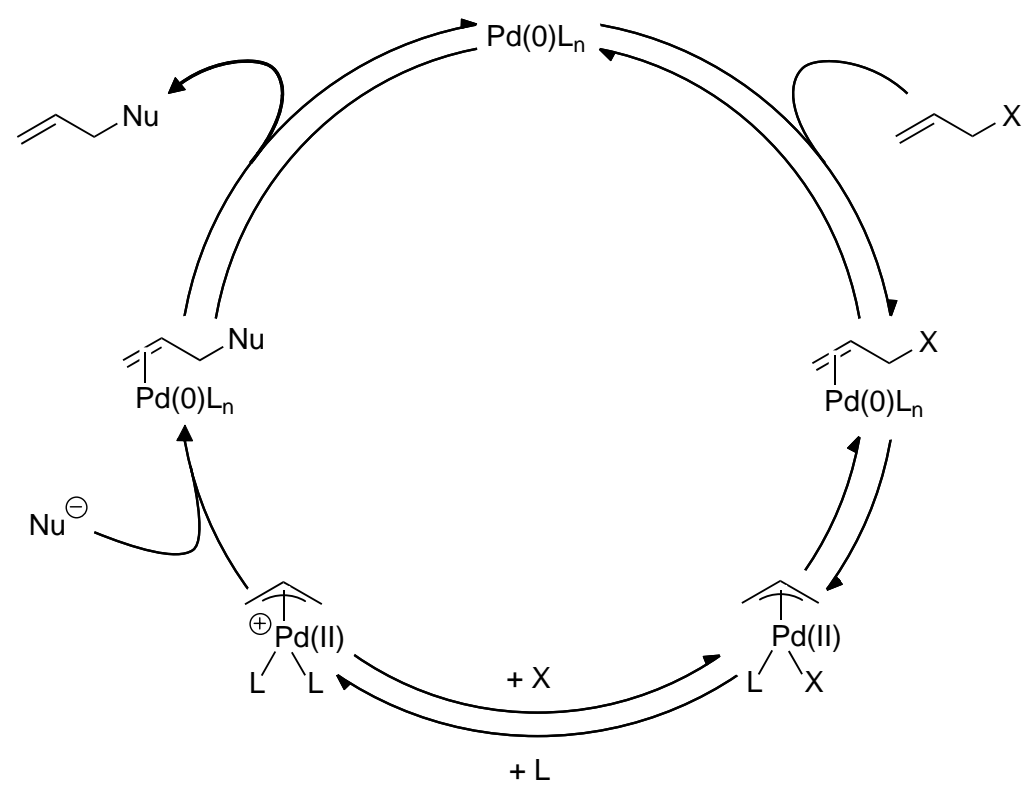

Schema 2.13: Mechanismus der Palladium-katalysierten allylischen Substitution.<smiles>CC(=O)OC(/C=C/c1ccccc1)c1ccccc1</smiles><smiles>CCOC(=O)C(C)C(C)I</smiles><smiles>COC(=O)C(C(=O)OC)c1ccccc1</smiles>

Schema 2.14: Palladium-katalysierte allylische Substitution von Dimethylmalonat mit einem Diphenylallylsystem. 


\section{Kapitel 3}

\section{Zielsetzung}

$N$-heterozyklische Carbene (NHCs) gewinnen in der Anorganischen Chemie immer mehr an Bedeutung, unter anderem wegen ihres ausgeprägten $\sigma$-Donorcharakters. Sie stellen aufgrund ihrer elektronischen und sterischen Eigenschaften eine äußerst interessante Ligandenklasse dar. Zu den großen Vorteilen der NHCs gehört die uneingeschränkte Möglichkeit zur Funktionalisierung des heterozyklischen Grundgerüstes, was zu ihrem verbreiteten Einsatz als Liganden führte. Durch geeignete Reaktionen kann unter anderem die Einführung chiraler Information vorgenommen werden. So lässt sich eine breite Palette solcher Verbindungen synthetisieren.

Darüber hinaus erwies sich Pyrazol, ein aromatischer Distickstoffheterozyklus, als ideale zentrale Einheit, welche die verbrückende Funktion in der Ligandsynthese übernimmt. Die Positionen 3 und 5 des Pyrazol-Bausteins sind in der Lage mit 1-funktionalisierten Imidazolium-Einheiten substituiert zu werden. Die Einführung einer Phenylgruppe an der Pyrazol-C ${ }^{4}$-Position sollte zu einer Verbesserung bezüglich der Löslichkeit sowie Kristallisationseigenschaften führen.

Aufgrund dieser Erkenntnisse wurde eine Verbindungsklasse mit Pyrazol-NHCHybridliganden entwickelt, welche nicht nur durch einen Chelateffekt eine Stabilisierung von Komplexen hervorrufen, sondern gleichzeitig durch das Einbringen zweier NHC-Einheiten zwei geeignete Bindungstaschen für Metallionen zu Verfügung stellen kann. Diese können zur Darstellung bimetallischer NHC-Komplexe, wie in Abbildung 3.1 gezeigt, verwendet werden, für die eine kooperative MehrzentrenReaktivität zu erwarten ist. 


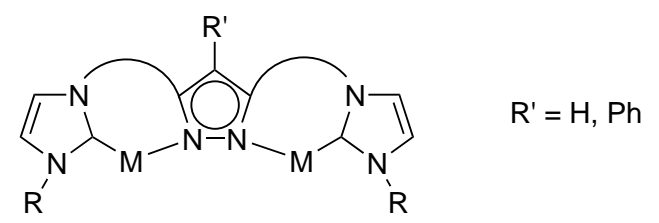

Abbildung 3.1: Allgemeines Bindungsmodell der Pyrazol-NHC-Hybridliganden.

Ziel der vorliegenden Arbeit war die Synthese eines Sortiments von Pyrazol-NHCHybridliganden. Die Ligandsysteme werden dabei durch aliphatische wie auch aromatische Substituenten am Imidazolyliden variiert. Von besonderem Interesse sind hierbei Seitenarme, die sperrige Substituenten tragen, welche ihrerseits die bimetallische Bindungstasche abschirmen. Außerdem sollen Substituenten ( $\left.\mathrm{R}^{\prime}\right)$ am Rückgrat des Pyrazols ( $\mathrm{C}^{4}$-Position) dabei variiert werden.

Durch Reaktionen mit unterschiedlichen Metallvorläufern können multinukleare Metallkomplexe dieser Liganden dargestellt werden, welche auf ihre Koordinationschemie hin untersucht werden sollten. Einen einfachen Zugang zu Übergangsmetall-NHC-Komplexen bietet die Methode der Transmetallierung, da die als Carbentransfer-Reagenzien verwendeten Silber-NHC-Komplexe in der Regel leicht aus den entsprechenden Imidazoliumsalzen und $\mathrm{Ag}_{2} \mathrm{O}$ zugänglich sind. Die Silber-NHC-Komplexe selbst sind von besonderem Interesse, u.a. aufgrund ihrer oft ungewöhnlichen strukturellen und optischen Eigenschaften.

Über die Methode der Silber-NHC-Komplex vermittelten Transmetallierung sollte so die Herstellung einer ganzen Reihe von Übergangsmetall-NHC-Komplexen ermöglicht werden. Weiterhin sollte der Synthese solcher Komplexe eine spektroskopische sowie strukturelle Charakterisierung folgen. Speziell durch Komplexierung der Liganden an geeignete Palladium(II)-Salze sollen Allylpalladiumkomplexe hergestellt werden und deren Anwendung als Katalysatoren in typischen Testreaktionen untersucht werden. Hervorzuheben sind dabei katalytische Allylübertragungsreaktionen, wie z.B. die allylische Alkylierung, da diese mechanistisch gut verstanden [112] und experimentell einfach realisierbar ist. Die zusätzliche Einführung von Chiralität in die Ligandsysteme eröffnet die Möglichkeit zur asymmetrischen Katalyse. 


\section{Kapitel 4}

\section{Ergebnisse und Diskussion}

\subsection{Synthese der Pyrazol-NHC-Liganden}

Die hygroskopischen Bis(imidazolium)-chloride $\left[\mathrm{H}_{4} \mathrm{~L}^{1}\right] \mathrm{Cl}_{3}-\left[\mathrm{H}_{4} \mathrm{~L}^{11}\right] \mathrm{Cl}_{3}$ wurden aus 3,5-Bis(chlormethyl)-1-(tetrahydropyran-2-yl)-1 $H$-pyrazol (6) bzw. 3,5-Bis(chlormethyl)-1-(tetrahydropyran-2-yl)-4-phenyl-1 $H$-pyrazol (12) mit einem Überschuss des jeweilligen $N$-substituierten Imidazols nach literaturbekannter Vorschrift synthetisiert (Schema 4.1). [35] Über einen nukleophilen Angriff des ImidazolStickstoffatoms auf das pseudo-benzylische Kohlenstoffatom wird das Chlorid abgespalten, bleibt aber als Gegenion erhalten. Durch Zugabe ethanolischer Salzsäure (siehe Schritt 2) wird die THP-Schutzgruppe abgespalten und das entsprechende Hydrochlorid $\left[\mathrm{H}_{4} \mathrm{~L}^{\mathrm{x}}\right] \mathrm{Cl}_{3}$ gebildet, welches durch Zugabe von Diethylether als weißer Feststoff ausfällt. Die THP-Gruppe wird hierbei auf das Ethanol übergetragen. $\mathrm{Zu}$ den Vorteilen dieser Synthesemethode gehört vor allem die Rückgewinnung des eingesetzten überschüssigen $N$-substituierten Imidazols.

Sämtliche nach dieser Methode synthetisierten pyrazolatverbrückten Bis(imidazolium)-Verbindungen wurden mit mäßigen bis guten Ausbeuten erhalten sowie durch NMR-Spektoskopie und Massenspektrometrie charakterisiert. Einkristalle, die für eine röntgenographische Analyse geeignet waren, konnten durch Diethylether-Diffusion erhalten werden. Exemplarisch ist die Festkörperstruktur von $\left[\mathrm{H}_{4} \mathrm{~L}^{6}\right] \mathrm{Cl}_{3}$ in Abbildung $4.1 \mathrm{zu}$ sehen. Der Feststoff ist stark hygroskopisch, wie nahezu alle Hydrochloride, weshalb keine zufriedenstellenden Elementaranalysen erhalten werden konnten.

Durch Zugabe von $\mathrm{NH}_{4} \mathrm{PF}_{6}$ oder $\mathrm{NaBF}_{4}$ zu den wässrigen Lösungen der Hydrochloride $\left[\mathrm{H}_{4} \mathrm{~L}^{\mathrm{x}}\right] \mathrm{Cl}_{3}$ wird die Isolierung der entsprechenden Hexafluorophosphat- 


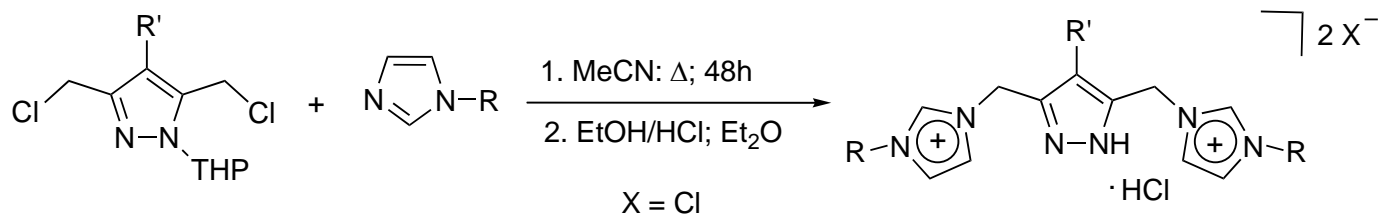

$\left[\mathrm{H}_{4} \mathrm{~L}^{1}\right] \mathrm{X}_{3}\left(\mathrm{R}=t \mathrm{Bu}, \mathrm{R}^{\prime}=\mathrm{H}\right)$

$\left[H_{4} L^{2}\right] X_{3}\left(R=t B u, R^{\prime}=P h\right)$

$\left[\mathrm{H}_{4} \mathrm{~L}^{3}\right] \mathrm{X}_{3}\left(\mathrm{R}=1\right.$-Adamantyl, $\left.\mathrm{R}^{\prime}=\mathrm{H}\right)$

$\left[\mathrm{H}_{4} \mathrm{~L}^{4}\right] \mathrm{X}_{3}\left(\mathrm{R}=1\right.$-Adamantyl, $\left.\mathrm{R}^{\prime}=\mathrm{Ph}\right)$

$\left[\mathrm{H}_{4} \mathrm{~L}^{5}\right] \mathrm{X}_{3}\left(\mathrm{R}=\right.$ Mes, $\left.\mathrm{R}^{\prime}=\mathrm{H}\right)$

$\left[\mathrm{H}_{4} \mathrm{~L}^{6}\right] \mathrm{X}_{3}\left(\mathrm{R}=\right.$ Mes, $\left.\mathrm{R}^{\prime}=\mathrm{Ph}\right)$

$\left[\mathrm{H}_{4} \mathrm{~L}^{7}\right] \mathrm{X}_{3}\left(\mathrm{R}=\right.$ 4-Nitrophenyl, $\left.\mathrm{R}^{\prime}=\mathrm{Ph}\right)$

$\left[H_{4} L^{8}\right] X_{3}\left(R=1\right.$-Benzyl, $\left.R^{\prime}=H\right)$

$\left[\mathrm{H}_{4} \mathrm{~L}^{9}\right] \mathrm{X}_{3}\left(\mathrm{R}=1\right.$-Benzyl, $\left.\mathrm{R}^{\prime}=\mathrm{Ph}\right)$

$\left[\mathrm{H}_{4} \mathrm{~L}^{\mathrm{L}}{ }^{10}\right] \mathrm{X}_{3}\left(\mathrm{R}=(\mathrm{S})\right.$-1-Phenylethyl, $\left.\mathrm{R}^{\prime}=\mathrm{H}\right)$

$\left[\mathrm{H}_{4} \mathrm{~L}^{11}\right] \mathrm{X}_{3}\left(\mathrm{R}=(\mathrm{S})-1\right.$-Phenylethyl, $\left.\mathrm{R}^{\prime}=\mathrm{Ph}\right)$

Schema 4.1: Synthese der Hydrochlorid-Liganden.

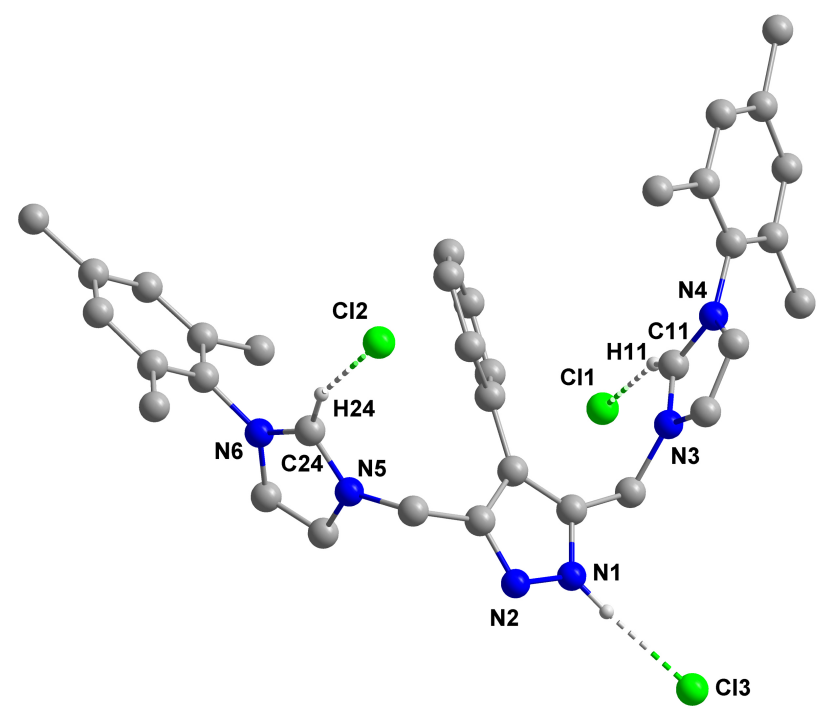

Abbildung 4.1: Festkörperstruktur von $\left[\mathrm{H}_{4} \mathrm{~L}^{6}\right] \mathrm{Cl}_{3}$. Die Wasserstoffbrückenbindungen sind hervorgehoben. Um die Darstellung übersichtlicher zu gestalten, wurden die nicht relevanten Wasserstoffatome und die Lösungsmittelmoleküle weggelassen. 
und Tetrafluoroborat-Salze ermöglicht (Schema 4.2). Diese werden bevorzugt als Liganden-Precursoren wegen ihrer erhöhten Löslichkeit gegenüber den entsprechenden Chloriden eingesetzt. Dadurch entstehen symmetrische Liganden des Typs $\left[\mathrm{H}_{3} \mathrm{~L}^{\mathrm{x}}\right]^{2+}$ mit chelatisierenden Seitenarmen. Die Variation des sterischen Anspruches der Seitenarme erlaubt den Zugang zu einer breiten Palette von pyrazolatverbrückten Bis(imidazolium)-Verbindungen, die sich zusätzlich durch die Substitution des $\mathrm{C}^{4}$-Atoms am Pyrazolring unterscheiden. Der Schwerpunkt dieser Arbeit richtet sich auf sperrige Substituenten R, welche die bimetallische Bindungstasche effektiv abschirmen können.
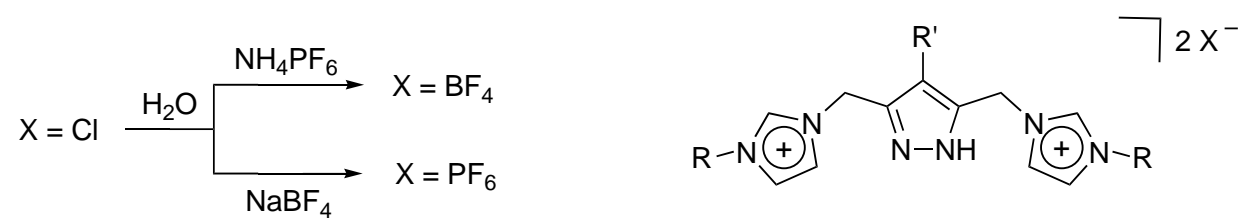

Schema 4.2: Umsalzen der Liganden mittels Zugabe von $\mathrm{NH}_{4} \mathrm{PF}_{6}$ oder $\mathrm{NaBF}_{4}$.

Die Verbindungen $\left[\mathrm{H}_{3} \mathrm{~L}^{\mathrm{x}}\right]\left(\mathrm{PF}_{6}\right)_{2}$ und $\left[\mathrm{H}_{3} \mathrm{~L}^{\mathrm{x}}\right]\left(\mathrm{BF}_{4}\right)_{2}$ zeigen (in Abhängigkeit von der Tautomerie des Pyrazol-NH) ein oder zwei charakteristische ${ }^{1} \mathrm{H}-\mathrm{NMR}$ Signale zwischen 9.0 und 9.5ppm (in DMSO- $d_{6}$ ). Diese stammen von dem aciden Imidazolium $\mathrm{CH}$-Proton $\left(\mathrm{CH}^{\operatorname{Im} 2}\right)$. Die verbrückenden $\mathrm{CH}_{2}$-Gruppen erscheinen als Singulett um $5.4 \mathrm{ppm}$, während die Pyrazol NH Protonen durch eine breite Resonanz bei etwa 13.3ppm charkterisiert sind. Sowohl ${ }^{1} \mathrm{H}$ - als auch ${ }^{13} \mathrm{C}-\mathrm{NMR}$ Spektren der Imidazolium Salze sind unabhängig vom jewewiligen Gegenion $\left(\mathrm{PF}_{6}^{-}\right.$vs. $\left.\mathrm{BF}_{4}^{-}\right)$. Die Erfahrung hat gezeigt dass die $\mathrm{BF}_{4}^{-}$-Salze bessere Kristallisationseigenschaften besitzen als die entsprechenden $\mathrm{PF}_{6}^{-}$-Salze.

Die Zusammensetzung von $\left[\mathrm{H}_{3} \mathrm{~L}^{1}\right]\left(\mathrm{BF}_{4}\right)_{2},\left[\mathrm{H}_{3} \mathrm{~L}^{3}\right]\left(\mathrm{BF}_{4}\right)_{2}$ und $\left[\mathrm{H}_{3} \mathrm{~L}^{4}\right]\left(\mathrm{PF}_{6}\right)_{2}$ wurde durch Röntgenkristallstrukturanalyse bestätigt. Exemplarisch hierzu sind in Abbildung 4.2 die Festkörperstrukturen von $\left[\mathrm{H}_{3} \mathrm{~L}^{1}\right]\left(\mathrm{BF}_{4}\right)_{2}$ und des mit einer Phenylgruppe am Rückgrat des Pyrazols funktionalisierten Analogons $\left[\mathrm{H}_{3} \mathrm{~L}^{2}\right]\left(\mathrm{BF}_{4}\right)_{2}$ dargestellt. Von Verbindung $\left[\mathrm{H}_{3} \mathrm{~L}^{2}\right]\left(\mathrm{BF}_{4}\right)_{2}$ sind feine, farblose Nadeln erhalten worden. Allerdings war die Qualität der Strukturbestimmung nicht ausreichend, um weitere Aussagen über die Bindungslängen und -winkel treffen zu können. Es konnte nur die Konstitution der Verbindung ermittelt werden. Ausgewählte Bindungslängen und -winkel von $\left[\mathrm{H}_{3} \mathrm{~L}^{1}\right]\left(\mathrm{BF}_{4}\right)_{2}$ gibt Tabelle 4.1 wieder. In allen Verbindungen wurden starke Wechselwirkungen zwischen den Imidazolium- $\mathrm{C}^{2} \mathrm{H}$ und/oder dem PyrazolProton und den entsprechenden Gegenionen gefunden. Die vorhandene Fehlordnung der Pyrazol-Einheit und der $\mathrm{BF}_{4}^{-}$-Anionen in $\left[\mathrm{H}_{3} \mathrm{~L}^{1}\right]\left(\mathrm{BF}_{4}\right)_{2}$ und $\left[\mathrm{H}_{3} \mathrm{~L}^{3}\right]\left(\mathrm{BF}_{4}\right)_{2}$ verhindern eine detaillierte Diskussion dieser Strukturen. 


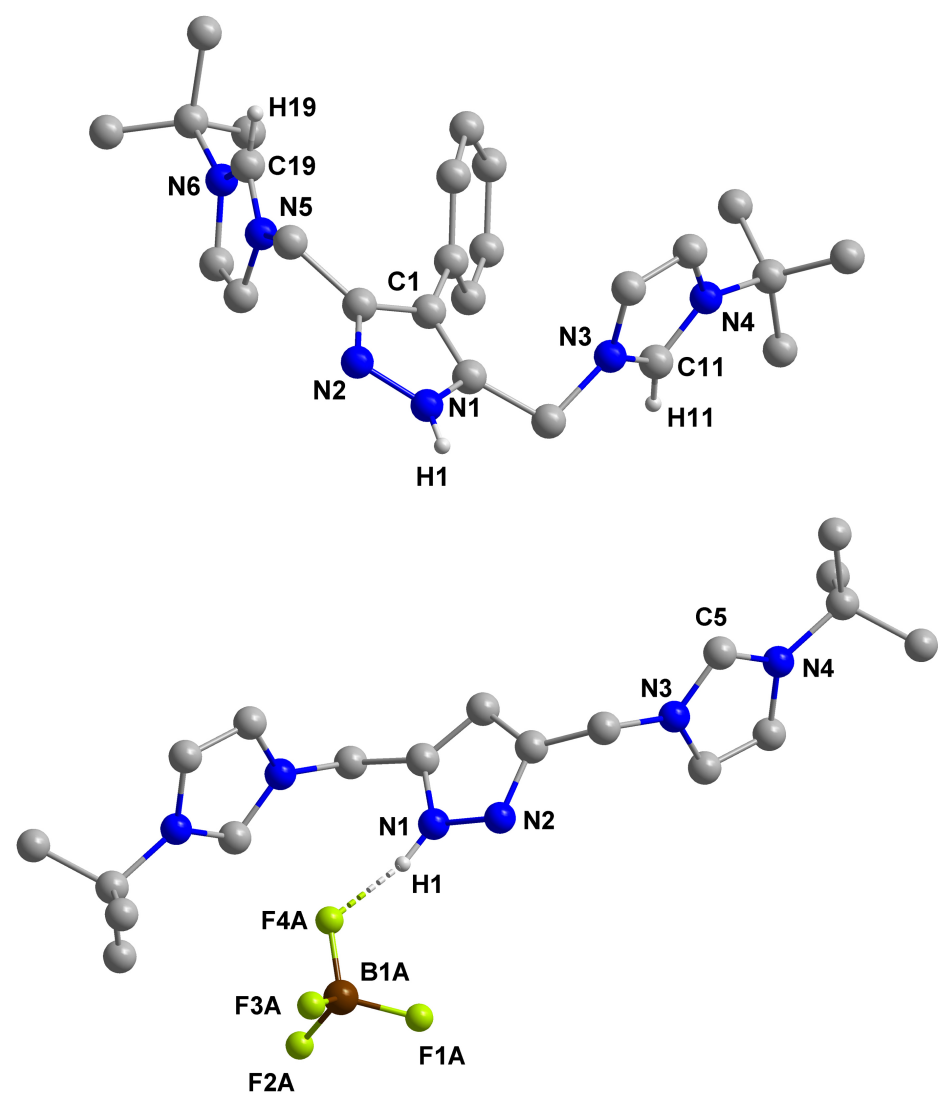

Abbildung 4.2: Konstitution von $\left[\mathrm{H}_{3} \mathrm{~L}^{2}\right]\left(\mathrm{BF}_{4}\right)_{2}$ (oben) und die Festkörperstruktur von $\left[\mathrm{H}_{3} \mathrm{~L}^{1}\right]\left(\mathrm{BF}_{4}\right)_{2}$ (unten). Um die Darstellung übersichtlicher zu gestalten, sind die nicht relevanten Wasserstoffatome und einige $\mathrm{BF}_{4}^{-}$-Ionen nicht eingezeichnet.

Tabelle 4.1: Ausgewählte Bindungslängen und -winkel von $\left[\mathrm{H}_{3} \mathrm{~L}^{1}\right]\left(\mathrm{BF}_{4}\right)_{2}$. Standardabweichungen sind in Klammern angegeben.

\begin{tabular}{l|cc}
\hline$\left[\mathrm{H}_{3} \mathrm{~L}^{1}\right]\left(\mathrm{BF}_{4}\right)_{2}$ & Bindungslänge $[\AA]$ & Winkel $\left[^{\circ}\right]$ \\
\hline $\mathrm{N} 3-\mathrm{C} 5$ & $1.328(3)$ & \\
$\mathrm{N} 4-\mathrm{C} 5$ & $1.325(3)$ & \\
$\mathrm{N} 1-\mathrm{N} 2$ & $1.343(6)$ & \\
$\mathrm{N} 3-\mathrm{C} 5-\mathrm{N} 4$ & & $109(2)$ \\
$\mathrm{C} 5-\mathrm{H} 5 \cdots \mathrm{F} 1 \mathrm{~A}$ & $3.278(3)$ & $166(3)$ \\
$\mathrm{C} 5-\mathrm{H} 5 \cdots \mathrm{F} 1 \mathrm{~B}$ & $3.02(2)$ & $148(3)$ \\
$\mathrm{N} 1-\mathrm{H} 1 \cdots \mathrm{F} 2 \mathrm{~A}$ & $3.315(5)$ & 118 \\
\hline
\end{tabular}


Eine Besonderheit stellt die Kristallstruktur von $\left[\mathrm{H}_{3} \mathrm{~L}^{4}\right]\left(\mathrm{PF}_{6}\right)_{2}$ dar (Abbildung 4.3). Diese wird vielmehr durch $\left[\mathrm{H}_{3} \mathrm{~L}^{4}\right]\left[\mathrm{H}_{2} \mathrm{~L}^{4}\right]\left(\mathrm{PF}_{6}\right)_{3}$ beschrieben, da es aus zwei verschiedenen Molekülen besteht, von denen eine am Pyrazol Stickstoffatom deprotoniert ist. Wasserstoffbrückenbindungen zwischen dem vollständig protonierten $\left[\mathrm{H}_{3} \mathrm{~L}^{4}\right]^{2+}$ und dem einfach deprotonierten $\left[\mathrm{H}_{2} \mathrm{~L}^{4}\right]^{+}$verursachen eine versetzte "face-to-face“ Anordnung beider Ligandenstränge mit einem zentralen $\mathrm{N}-\mathrm{H} \cdots \mathrm{H}$ und zwei flankierenden $\mathrm{C}^{2}-\mathrm{H} \cdots \mathrm{N}$ Wechselwirkungen. Die Einbeziehung eines Imidazolium-C ${ }^{2}$ Wasserstoffatoms in die Wasserstoffbrückenbindung in Richtung eines Pyrazol-N ist ungewöhnlich, da Pyrazole üblicherweise via N-HN.N Wasserstoffbrückenbindungen wechselwirken. $[42,116]$ Die Gesamtladung von +3 wird durch die drei $\mathrm{PF}_{6}^{-}$-Anionen kompensiert. Eines der verbleibenden peripheren Imidazolium- $\mathrm{C}^{2}$ Wasserstoffatome zeigt in Richtung eines $\mathrm{PF}_{6}^{-}$-Anions. Die $\mathrm{C}(-\mathrm{H}) \cdots \mathrm{F}$ Bindungslänge liegt im Bereich der für diese schwache Wechselwirkung bereits bekannten Abstände. [117] Ausgewählte Bindungslängen und Winkel dieser Verbindung sind in Tabelle 4.2 zusammengefasst.

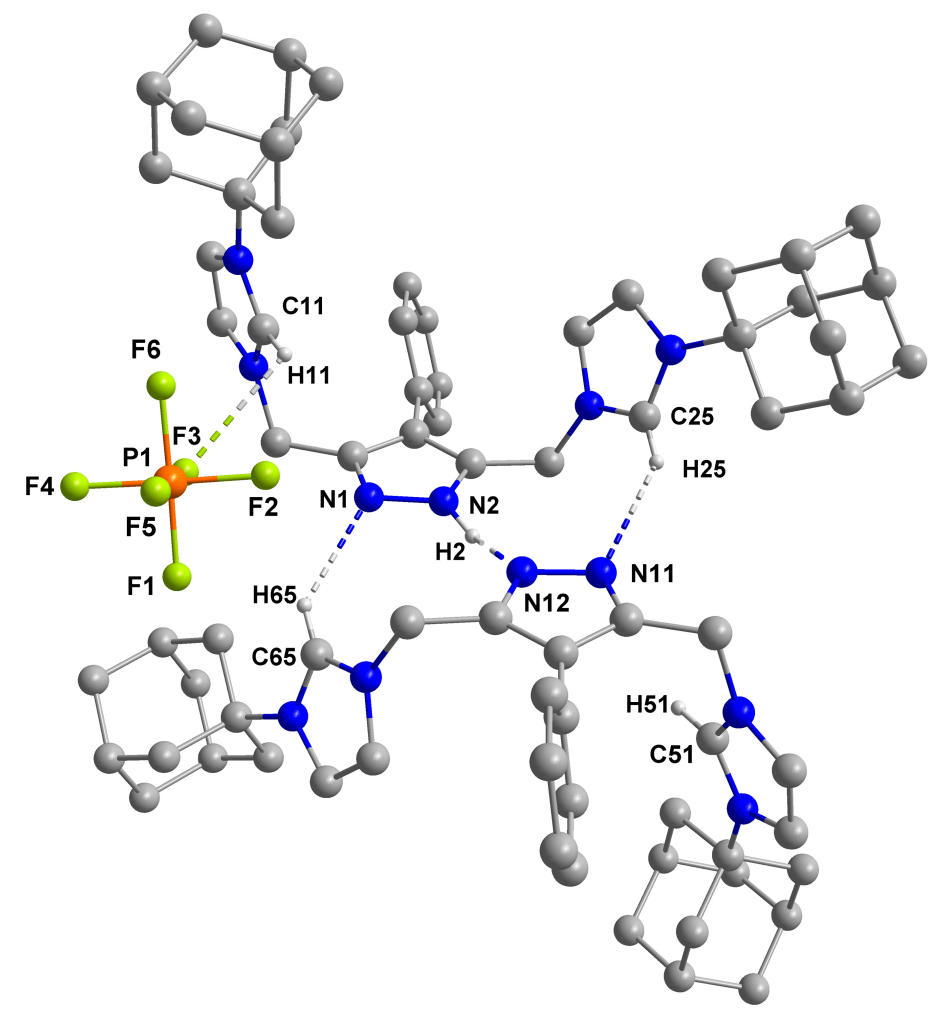

Abbildung 4.3: Festkörperstruktur von $\left[\mathrm{H}_{3} \mathrm{~L}^{4}\right]\left[\mathrm{H}_{2} \mathrm{~L}^{4}\right]\left(\mathrm{PF}_{6}\right)_{3}$. Um die Darstellung übersichtlicher zu gestalten, sind die nicht relevanten Wasserstoffatome, die Lösungsmittelmoleküle und teilweise die Gegenionen nicht gezeigt.

Die Verbindung kristallisiert in der Raumgruppe $P 1$. Es ist auch möglich die Struktur von $\left[\mathrm{H}_{3} \mathrm{~L}^{4}\right]\left[\mathrm{H}_{2} \mathrm{~L}^{4}\right]\left(\mathrm{PF}_{6}\right)_{3}$ der Raumgruppe $P \overline{1}$ zuzuordnen. In diesem Fall sind 
Tabelle 4.2: Ausgewählte Bindungslängen und -winkel von $\left[\mathrm{H}_{3} \mathrm{~L}^{4}\right]\left[\mathrm{H}_{2} \mathrm{~L}^{4}\right]\left(\mathrm{PF}_{6}\right)_{3}$. Standardabweichungen sind in Klammern angegeben.

\begin{tabular}{l|cc}
\hline$\left[\mathrm{H}_{3} \mathrm{~L}^{4}\right]\left[\mathrm{H}_{2} \mathrm{~L}^{4}\right]\left(\mathrm{PF}_{6}\right)_{3}$ & Bindungslänge $[\AA]$ & Winkel $\left[^{\circ}\right]$ \\
\hline $\mathrm{N} 3-\mathrm{C} 11$ & $1.330(4)$ & \\
$\mathrm{N} 4-\mathrm{C} 11$ & $1.325(4)$ & \\
$\mathrm{N} 1-\mathrm{N} 2$ & $1.345(3)$ & \\
$\mathrm{N} 3-\mathrm{C} 11-\mathrm{N} 4$ & & $109.3(2)$ \\
$\mathrm{N} 2-\mathrm{H} 2 \cdots \mathrm{N} 12$ & $2.636(3)$ & $155(5)$ \\
$\mathrm{C} 11-\mathrm{H} 11 \cdots \mathrm{F} 3$ & $3.182(4)$ & $107(4)$ \\
$\mathrm{C} 25-\mathrm{H} 25 \cdots \mathrm{N} 11$ & $3.256(4)$ & $140(5)$ \\
$\mathrm{C} 65-\mathrm{H} 65 \cdots \mathrm{N} 1$ & $3.197(4)$ & $137(5)$ \\
\hline
\end{tabular}

die $\mathrm{PF}_{6}^{-}$-Gegenionen und das Wasserstoffatom am Pyrazol-Stickstoffatom fehlgeordnet. Aus diesem Grund schien die Einordung dieser Struktur in die P1 Raumgruppe chemisch sinnvoller. Die korrekte absolute Struktur von $\left[\mathrm{H}_{3} \mathrm{~L}^{4}\right]\left[\mathrm{H}_{2} \mathrm{~L}^{4}\right]\left(\mathrm{PF}_{6}\right)_{3}$ konnte nicht zuverlässig bestimmt werden. Sowohl Wasser als auch Acetonitril waren in der Molekülstruktur fehlgeordnet. Es konnten keine Wasserstoffatome gefunden werden, die an die Sauerstoffatome des Wassers gebunden waren.

Zusätzlich wurde versucht, neben den arylischen und aliphatischen Substituenten auch Substituenten mit chiraler Information in den Liganden einzuführen. Nach der gleichen Synthesestrategie konnte aus der Reaktion von Pyrazol (6) mit dem entspechenden chiralen Imidazol-Seitenarm und anschließender Salzmetathese Verbindung $\left[\mathrm{H}_{3} \mathrm{~L}^{10}\right]\left(\mathrm{BF}_{4}\right)_{2}$ synthetisiert werden. Erwartungsgemäß haben sich die Verschiebungen der Signale im ${ }^{1} \mathrm{H}-\mathrm{NMR}$ Spektrum von $\left[\mathrm{H}_{3} \mathrm{~L}^{10}\right]\left(\mathrm{BF}_{4}\right)_{2}$ im Vergleich zu denen von $\left[\mathrm{H}_{4} \mathrm{~L}^{10}\right]\left(\mathrm{Cl}_{3}\right)$ vor der Salzmetathese nicht stark verändert. $\left[\mathrm{H}_{3} \mathrm{~L}^{10}\right]\left(\mathrm{BF}_{4}\right)_{2}$ zeigt zwei Signale zwischen 9.3 und $9.5 \mathrm{ppm}$ (in DMSO- $d_{6}$ ); diese stammen von dem aciden Imidazolium-CH-Proton $\left(\mathrm{CH}^{\operatorname{Im} 2}\right)$. Mangels Auflösung ist die Aufspaltung der $\mathrm{CH}^{I m 2}$-Gruppe zu einem Dublett vom Dublett auf Grund der Kopplung zu den beiden anderen Protonen des Imidazols $\mathrm{CH}^{I m}$ in Position 4 und 5, die zu erwarten wäre, nicht sichtbar (eine Beobachtung, die für alle bisher synthetisierten $\left[\mathrm{H}_{3} \mathrm{~L}^{\mathrm{x}}\right](\mathrm{X})_{2} ; \mathrm{X}=\mathrm{BF}_{4}, \mathrm{PF}_{6}$ gilt). Aufgrund der Tautomerie des Pyrazol-NH sind zwei ${ }^{1} \mathrm{H}-\mathrm{NMR}$ Signale zwischen 5.4 und $5.5 \mathrm{ppm}$ (in DMSO- $d_{6}$ ) zu sehen, welche zu den verbrückenden $\mathrm{CH}_{2}$-Gruppen gehören. Charakteristisch für diese Verbindung ist das Signal des Seitenarm-CH-Protons bei etwa 5.8ppm, welches zum Quartett aufspaltet. Das Pyrazol-NH-Proton ist bei etwa $13.3 \mathrm{ppm}$ zu beobachten. Im ${ }^{13} \mathrm{C}$ NMR Spektrum ist bei einer Verschiebung von 105ppm das charakteristische Signal 
für das $\mathrm{CH}^{P z}$ zu sehen. Sowohl ${ }^{1} \mathrm{H}$ - als auch ${ }^{13} \mathrm{C}-\mathrm{NMR}$ Spektren zeigen deutliche Ähnlichkeiten zu denen von achiralen Liganden.

Die Darstellung des $\mathrm{C}^{4}$-Phenyl substituierten Pyrazols wurde ebenfalls vorgenommen. Es gelang die Verbindung $\left[\mathrm{H}_{3} \mathrm{~L}^{11}\right]\left(\mathrm{BF}_{4}\right)_{2}$ in nahezu quantitativer Ausbeute zu synthetisieren und zu kristallisieren. Die erhaltenen Kristalle konnten röntgenkristallographisch untersucht werden und ergaben die Molekülstruktur, die in Abbildung 4.2 dargestellt ist. Die Verbindung kristallisiert in der Raumgruppe $P 2_{1} 2_{1} 2_{1}$. Der gefundene Winkel zwischen Pyrazol und der Phenylgruppe beträgt etwa $127^{\circ}$. Weitere ausgewählte Bindungslängen und -winkel von Verbindung $\left[\mathrm{H}_{3} \mathrm{~L}^{11}\right]\left(\mathrm{BF}_{4}\right)_{2}$ sind in Tabelle 4.3 aufgelistet.

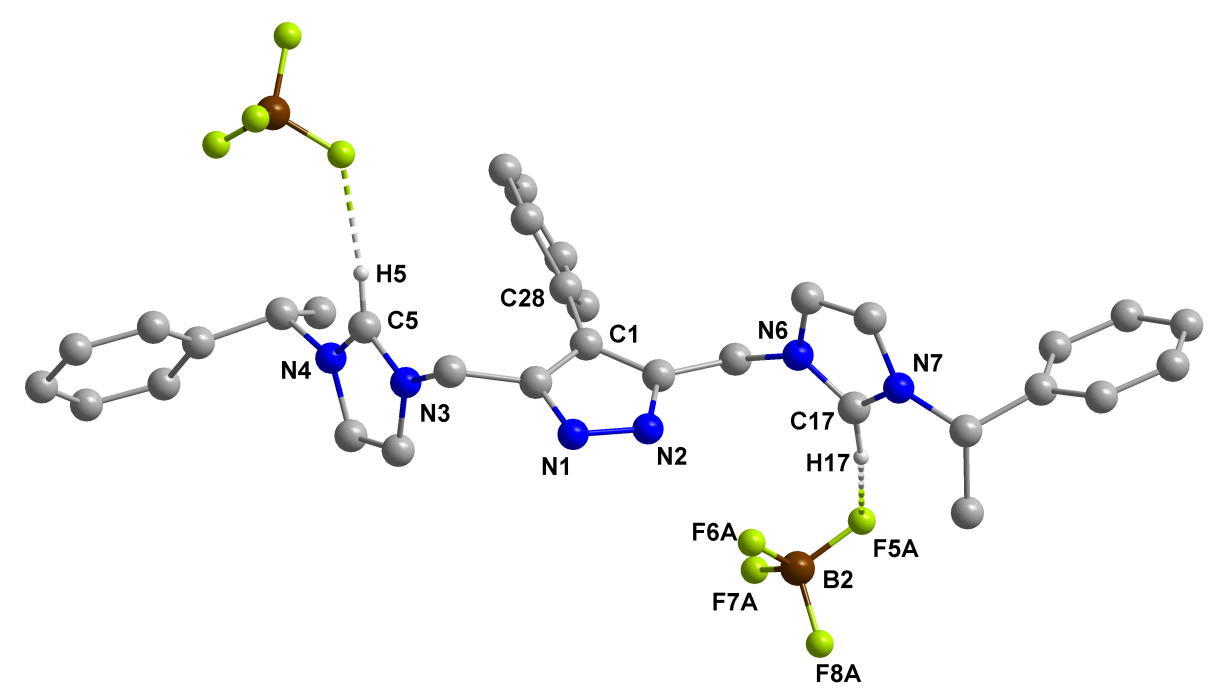

Abbildung 4.4: Festkörperstruktur von $\left[\mathrm{H}_{3} \mathrm{~L}^{11}\right]\left(\mathrm{BF}_{4}\right)_{2}$. Um die Darstellung übersichtlicher zu gestalten, sind die nicht relevanten Wasserstoffatome nicht gezeigt.

Im Allgemeinen wird in der Literatur angenommen, dass $\mathrm{C}-\mathrm{H} \cdots \mathrm{F}^{-}$Wechselwirkungen den üblichen $\mathrm{N} / \mathrm{O}-\mathrm{H} \cdots \mathrm{X}^{-}$Wasserstoffbrückenbindungen ähneln, aber schwächer als diese sind. Die C-H... ${ }^{-}$Wechselwirkung liegt in dieser Struktur mit etwa $3.1 \AA$ im erwarteten Bereich für den Donor-Akzeptor-Abstand. [118] Die Abstände der C $\cdots$ F in sämtlichen Verbindungen dieser Arbeit liegen in dem für Wasserstoffbrückenbindungen typischen Bereich. [49, 117] In der Literatur sind Daten über den Winkel am Wasserstoffatom zu finden, welcher größer als $140^{\circ}$ ist. Die $\mathrm{BF}_{4}^{-}$Gegenionen besitzen in diesem Fall eine Fehlordnung, die eine genauere Aussage über die $\mathrm{H} \cdots \mathrm{FBF}_{3}^{-}$Wechselwirkungen erschwert. Weitere Kristallstrukturdaten dieser Verbindung sind in Kapitel 7 zusammengefasst. 
Tabelle 4.3: Ausgewählte Bindungslängen und -winkel von $\left[\mathrm{H}_{3} \mathrm{~L}^{11}\right]\left(\mathrm{BF}_{4}\right)_{2}$. Standardabweichungen sind in Klammern angegeben.

\begin{tabular}{l|cc}
\hline$\left[\mathrm{H}_{3} \mathrm{~L}^{11}\right]\left(\mathrm{BF}_{4}\right)_{2}$ & Bindungslänge $[\AA]$ & Winkel $\left[^{\circ}\right]$ \\
\hline $\mathrm{N} 3-\mathrm{C} 5$ & $1.329(5)$ & \\
$\mathrm{N} 4-\mathrm{C} 5$ & $1.326(5)$ & \\
$\mathrm{N} 1-\mathrm{N} 2$ & $1.350(5)$ & \\
$\mathrm{N} 3-\mathrm{C} 5-\mathrm{N} 4$ & & $108.1(4)$ \\
$\mathrm{C} 2-\mathrm{C} 1-\mathrm{C} 28$ & & $127.8(3)$ \\
$\mathrm{N} 2-\mathrm{H} 2 \cdots \mathrm{F} 1 \mathrm{~B}$ & $2.82(3)$ & $140(5)$ \\
$\mathrm{N} 2-\mathrm{H} 2 \cdots \mathrm{F} 3 \mathrm{~A}$ & $2.955(8)$ & $141(5)$ \\
$\mathrm{C} 5-\mathrm{H} 5 \cdots \mathrm{F} 1 \mathrm{~A}$ & $3.047(8)$ & $174(6)$ \\
$\mathrm{C} 5-\mathrm{H} 5 \cdots \mathrm{N} 1 \mathrm{~B}$ & $3.10(3)$ & $164(6)$ \\
$\mathrm{C} 17-\mathrm{H} 17 \cdots \mathrm{F} 5 \mathrm{~A}$ & $3.064(6)$ & $159(5)$ \\
$\mathrm{C} 17-\mathrm{H} 17 \cdots \mathrm{F} 5 \mathrm{~B}$ & $3.146(16)$ & $139(5)$ \\
\hline
\end{tabular}

\subsection{Synthese der Komplexe}

Die in dieser Arbeit neu dargestellten Liganden sowie die bereits bekannten Liganden wurden für die Synthese von Metallkomplexen eingesetzt. Als erstes wird über eine einfache Methode zur Synthese von Silber-NHC-Komplexen berichtet. Diese eignen sich als Vorläufer-Verbindungen für die Synthese anderer ÜbergangsmetallNHC-Komplexe. Die Herstellung und Eigenschaften bi- und multimetallischer NHCKomplexe mit ausgewählten Metallen $(\mathrm{Ag}, \mathrm{Pd}, \mathrm{Ni}$ und $\mathrm{Cu}$ ) werden in diesem $\mathrm{Ab}$ schnitt vorgestellt.

\subsubsection{Silber-NHC-Komplexe}

Die Reaktion von Imidazoliumsalzen mit Silber(I)oxid in Acetonitril führt in der Regel zu den tetranuklearen $\mathrm{Ag}(\mathrm{I})$-NHC-Komplexen $\left[\left(\mathrm{L}^{\mathrm{x}}\right)_{2} \mathrm{Ag}_{4}\right]^{2+}$. Die Synthese erfolgt an Luft durch Rühren (2 Tage) bei Raumtemperatur. Anschließend wird nicht umgesetztes $\mathrm{Ag}_{2} \mathrm{O}$ abfiltriert. In dieser Arbeit wurde die Methode in leicht abgeänderter Weise angewendet [59], da die erhaltenen Ausbeuten nicht zufriedenstellend waren. Bessere Ausbeuten (bis zu 64\%, je nach Substituenten R und R') konnten über die Methode der in situ Salzmetathese, wie in der Literatur berichtet [86], erzielt werden. Es muss außerdem darauf geachtet werden, dass die Reaktionen unter Lichtausschluss stattfinden, da Silberverbindungen in der Regel unter Lichteinfluss zur Zersetzung tendieren. Es hat sich jedoch herausgestellt, dass die 
Mehrheit der synthetisierten Ag-Komplexe sowohl als Feststoff als auch in gelöster Form stabil bleiben. Falls das Zerfallen der Verbindungen in Lösung eintrifft, macht sich dies nach einiger Zeit durch eine Trübung der jeweiligen Lösung bzw. die Bildung eines Silberspiegels bemerkbar, der sich auf der Gefäßwand abscheidet. Das Ausbleiben oder Eintreten der Zersetzung wurde zusätzlich mit Hilfe von NMR-Untersuchungen bestätigt. Exemplarisch sind in Abbildung 4.5 die Festkörperstrukturen von $\left[\left(\mathrm{L}^{1}\right)_{2} \mathrm{Ag}_{4}\right]\left(\mathrm{BF}_{4}\right)_{2}$ und $\left[\left(\mathrm{L}^{2}\right)_{2} \mathrm{Ag}_{4}\right]\left(\mathrm{BF}_{4}\right)_{2}$ gezeigt. In Tabelle 4.4 sind charakteristische Bindungslängen und -winkel beider Verbindungen aufgeführt.

Tabelle 4.4: Ausgewählte Bindungslängen und -winkel von $\left[\left(\mathrm{L}^{1}\right)_{2} \mathrm{Ag}_{4}\right]\left(\mathrm{BF}_{4}\right)_{2}$ und $\left[\left(\mathrm{L}^{2}\right)_{2} \mathrm{Ag}_{4}\right]\left(\mathrm{BF}_{4}\right)_{2}$. Standardabweichungen sind in Klammern angegeben.

\begin{tabular}{l|cc}
\hline$\left[\left(\mathrm{L}^{1}\right)_{2} \mathrm{Ag}_{4}\right]\left(\mathrm{BF}_{4}\right)_{2}$ & Bindungslänge $[\AA]$ & Winkel $\left[^{\circ}\right]$ \\
\hline $\mathrm{Ag} 1-\mathrm{C} 13^{\prime}$ & $2.078(5)$ & \\
$\mathrm{Ag} 1-\mathrm{N} 1$ & $2.097(5)$ & \\
$\mathrm{Ag} 1-\mathrm{Ag} 2$ & $3.1943(6)$ & \\
$\mathrm{C} 13^{\prime}-\mathrm{Ag} 1-\mathrm{N} 1$ & & $178.22(19)$ \\
$\mathrm{C} 13^{\prime}-\mathrm{Ag} 1-\mathrm{Ag} 2$ & & $110.59(15)$ \\
\hline \hline$\left[\left(\mathrm{L}^{2}\right)_{2} \mathrm{Ag}{ }_{4}\right]\left(\mathrm{BF}_{4}\right)_{2}$ & & \\
\hline $\mathrm{Ag} 1-\mathrm{Ag} 4$ & $2.9255(8)$ & \\
$\mathrm{Ag} 2-\mathrm{Ag} 3$ & $3.2717(9)$ & \\
$\mathrm{Ag} 1-\mathrm{C} 55$ & $2.055(3)$ & \\
$\mathrm{Ag} 1-\mathrm{N} 1$ & $2.084(2)$ & $176.70(10)$ \\
$\mathrm{C} 55-\mathrm{Ag} 1-\mathrm{N} 1$ & & $102.41(8)$ \\
$\mathrm{C} 55-\mathrm{Ag} 1-\mathrm{Ag} 4$ & & $74.37(6)$ \\
$\mathrm{N} 1-\mathrm{Ag} 1-\mathrm{Ag} 4$ & & \\
\hline
\end{tabular}

$\left[\left(\mathrm{L}^{1}\right)_{2} \mathrm{Ag}_{4}\right]^{2+},\left[\left(\mathrm{L}^{3}\right)_{2} \mathrm{Ag}_{4}\right]^{2+}$ und $\left[\left(\mathrm{L}^{4}\right)_{2} \mathrm{Ag}_{4}\right]^{2+}$ sind Beispiele für ein wiederkehrendes Strukturmotiv, welches bei Silberkomplexen mit ähnlichen Liganden, die NHCs und Pyrazol-Einheiten verbinden, beobachtet werden. Allerdings wurden bisher nur wenige dieser Komplexe strukturell charakterisiert. Diese Arbeit bestätigt, dass diese tetranuklearen Cluster auch im Falle von sperrigen Imidazol-Substituenten wie tert-Butyl oder 1-Adamantyl gebildet werden. Alle (drei) Komplexe bestehen aus vier Silberatomen und zwei Liganden und besitzen eine kristallographische $\mathrm{C}_{i}$ Symmetrie. Die verbleibende positive Ladung wird entweder durch $\mathrm{PF}_{6}^{-}$- oder $\mathrm{BF}_{4}^{-}$Anionen kompensiert. Die Anordnung der Silberatome ist nahezu rechteckig.

In den letzten Jahren wurden etliche Beispiele für schwache Wechselwirkungen in $\mathrm{d}^{10}$ Münzmetall-Komplexen gefunden. [119-122] Für alle Ag-Komplexe $\left[\left(\mathrm{L}^{\mathrm{x}}\right)_{2} \mathrm{Ag}_{4}\right]^{2+}$ ist eine derartige closed-shell Wechselwirkung recht schwach oder gar nicht vorhanden, da die Ag...Ag Abstände weit über 3.1 ̊ liegen. [121, 122] Trotz des 

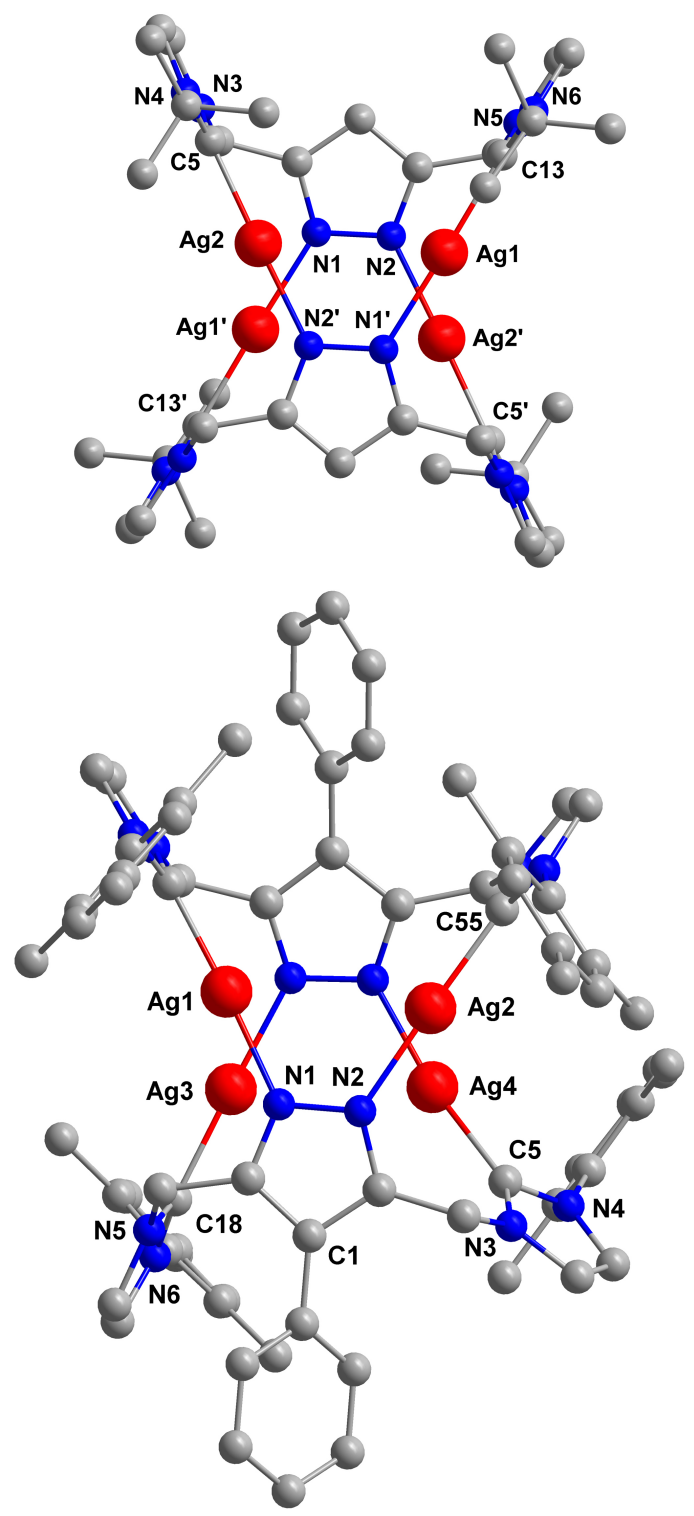

Abbildung 4.5: Festkörperstrukturen der $\mathrm{Ag}(\mathrm{I})$-NHC-Komplexe $\left[\left(\mathrm{L}^{1}\right)_{2} \mathrm{Ag}_{4}\right]\left(\mathrm{BF}_{4}\right)_{2}$ (oben) und $\left[\left(\mathrm{L}^{2}\right)_{2} \mathrm{Ag}_{4}\right]\left(\mathrm{BF}_{4}\right)_{2}$ (unten). Aus Gründen der Übersichtlichkeit sind die Wasserstoffatome und die jeweilligen Gegenionen nicht dargestellt. Symmetrieoperationen für äquivalente Atome: (') $-\mathrm{x}+1,-\mathrm{y}+1,-\mathrm{z}+1$. 
hohen sterischen Anspruchs der peripheren tert-Butyl- bzw. 1-Adamantyl-Gruppen liegen die Ag‥Ag Abstände in der gleichen Größenordnung wie bei dem sterisch weniger anspruchsvollen Silber(I)-Komplex von 3,5-Bis[methylimidazolium-1ylmethyl]1H-pyrazol. Dies deutet darauf hin, dass die tert-Butyl oder 1-Adamantyl Substituenten keine Auswirkung auf die $\mathrm{Ag}_{4}$-Grundeinheit haben. Eine noch genauere Betrachtung zeigt die interessante Tatsache, dass zwei offenkundig unterschiedliche Ag...Ag Wechselwirkungen in allen Komplexen dieser Art beobachet werden. In $\left[\left(\mathrm{L}^{1}\right)_{2} \mathrm{Ag}_{4}\right]^{2+}$ ist dieser Unterschied etwa $0.2 \AA$, während der Unterschied in $\left[\left(\mathrm{L}^{3}\right){ }_{2} \mathrm{Ag}_{4}\right]^{2+}$ und $\left[\left(\mathrm{L}^{4}\right){ }_{2} \mathrm{Ag}_{4}\right]^{2+}$ bei etwa $0.1 \AA$ liegt. Ein Unterschied in den $\mathrm{Ag} \cdots \mathrm{Ag}$ Abständen von fast $0.4 \AA$ wurde für den Silber(I)-Komplex von 3,5-Bis[3-(2,4,6trimethylphenyl)imidazolium-1-ylmethyl] $1 H$-pyrazol gefunden, was möglicherweise auf die paarweise $\mathrm{d}^{10}-\mathrm{d}^{10}$ Wechselwirkung in diesem Komplex hindeutet. [121, 122] Offenbar ist dieser Effekt im Falle von $\left[\left(\mathrm{L}^{1}\right)_{2} \mathrm{Ag}_{4}\right]^{2+},\left[\left(\mathrm{L}^{3}\right)_{2} \mathrm{Ag}_{4}\right]^{2+}$ und $\left[\left(\mathrm{L}^{4}\right)_{2} \mathrm{Ag}_{4}\right]^{2+}$ weniger ausgeprägt. Die Bindungslängen der Ag-C und Ag-N Bindungen (mit etwa $2.1 \AA$ ) sowie der Bindungswinkel bei der fast linearen N-Ag-C-Einheit liegen wie erwartet im entsprechenden Bereich. Der Winkel von N-C-N beträgt etwa $105^{\circ}$ und ist somit etwas kleiner als bei den protonierten Imidazoliumsalzen (ca. 109²).

Das auffälligste Merkmal in den ${ }^{1} \mathrm{H}-\mathrm{NMR}$ Spektren der Ag-Komplexe $\left[\left(\mathrm{L}^{1}\right)_{2} \mathrm{Ag}_{4}\right]\left(\mathrm{BF}_{4}\right)_{2},\left[\left(\mathrm{~L}^{3}\right)_{2} \mathrm{Ag}_{4}\right]\left(\mathrm{BF}_{4}\right)_{2}$ und $\left[\left(\mathrm{L}^{4}\right)_{2} \mathrm{Ag}_{4}\right]\left(\mathrm{PF}_{6}\right)_{2}$ ist die Abwesenheit der $\mathrm{CH}^{\operatorname{Im} 2}$ Resonanz, welche daraus resultiert, dass das Imidazoliumproton durch eine Silber-Carbenbindung ersetzt wurde. Des Weiteren erscheinen die verbrückenden $\mathrm{CH}_{2}$-Gruppen als AB-System. Im ${ }^{13} \mathrm{C}-\mathrm{NMR}$ Sprektrum von $\left[\left(\mathrm{L}^{1}\right)_{2} \mathrm{Ag}_{4}\right]\left(\mathrm{BF}_{4}\right)_{2}$ erscheint das Carbenkohlenstoffatom als breites Singulett (175ppm) mit einer Tieffeldverschiebung von 40ppm im Vergleich zum freien Imidazoliumsalz. Diese Peakverbreiterung deutet möglicherweise darauf hin, dass die Kohlenstoff-Silber-Bindung in Lösung nicht dauerhaft besteht; zwei schmale Dubletts mit ${ }^{1} J\left({ }^{13} \mathrm{C}^{107} \mathrm{Ag}\right)=235 \mathrm{~Hz}$ und ${ }^{1} J\left({ }^{13} C^{109} A g\right)=271 \mathrm{~Hz}$ wurden nur bei einer Temperatur von $-25^{\circ} \mathrm{C}$ beobachtet. Ähnlich verhält es sich mit der Pyrazol- ${ }^{15} \mathrm{~N}-$ Resonanz $(-113 \mathrm{ppm})$, dessen Signal um etwa 20ppm tieffeldverschoben ist, welches sich bei tiefer Temperatur in zwei Signale mit einer ${ }^{1} J\left({ }^{15} N^{107} \mathrm{Ag}\right)$-Kopplungskonstante von $73 \mathrm{~Hz}$ aufteilt, die zwei unterschiedlichen Isotopen zuzuordnen sind. Die ${ }^{109} \mathrm{Ag}$ Resonanz wird durch die J-Kopplung mit Pyrazol, Imidazol und $\mathrm{CH}_{2}$ Protonen detektiert und erscheint bei $634 \mathrm{ppm}$. Chemische Verschiebung und Kopplungskonstanten stimmen mit denen von zuvor untersuchten Pyrazolat-NHC Komplexen überein. [79]

Wenn die Reaktion von $\left[\mathrm{H}_{3} \mathrm{~L}^{1}\right]\left(\mathrm{PF}_{6}\right)_{2}$ und Silber(I)oxid in Aceton anstelle von Acetonitril durchgeführt wird, wird das Produkt $\left[\left(\mathrm{H}_{2} \mathrm{~L}^{1}\right)_{4} \mathrm{Ag}_{4}\right]\left(\mathrm{PF}_{6}\right)_{8}$ erhalten, welches vier Liganden und vier Silberatome enthält. Die ${ }^{1} \mathrm{H}-$ und ${ }^{13} \mathrm{C}-\mathrm{NMR}$ Spektren dieses neuartigen Komplexes unterscheiden sich deutlich von denen des $\left[\left(\mathrm{L}^{1}\right)_{2} \mathrm{Ag}_{4}\right]^{2+}$ : Die 
${ }^{1} \mathrm{H}-\mathrm{NMR}$ Signale für die verbrückenden $\mathrm{CH}_{2}$-Gruppen ergeben ein Singulett und der Peak bei 8.56ppm zeigt, dass die Imidazole nicht durch Silber koordiniert sind. NOE Korrelationen zwischen den Pyrazol- und $\mathrm{CH}_{2}$-Protonen auf der einen Seite und den nahegelegenen und isolierten CH-Protonen auf der anderen Seite zeigen, dass die Imidazolgruppen in Lösung völlige Bewegungsfreiheit besitzen. Des Weiteren bleibt bis zu einer Temperatur von $-40^{\circ} \mathrm{C}$ die Pyrazol ${ }^{15} \mathrm{~N}$ Resonanz ein Singulett und die ${ }^{109} \mathrm{Ag}$ Resonanz unmessbar, was darauf hindeutet, dass die Stickstoff-Silber Bindung hoch dynamisch ist. Kristallisation durch langsame Diethylether-Diffusion in eine Lösung von $\left[\left(\mathrm{H}_{2} \mathrm{~L}^{1}\right)_{4} \mathrm{Ag}_{4}\right]\left(\mathrm{PF}_{6}\right)_{8}$ in Aceton bei Raumtemperatur ergab farblose Kristalle, deren Struktur durch Röntgenkristallstrukturanalyse bestimmt werden konnte (Abbildung 4.6 (oben)). Wie in Abbildung 4.6 (unten) zu sehen ist, besitzt das Molekül eine sattelförmige Struktur.

$\left[\left(\mathrm{H}_{2} \mathrm{~L}^{1}\right)_{4} \mathrm{Ag}_{4}\right]\left(\mathrm{PF}_{6}\right)_{8}$ besitzt einen zentralen 12-gliedrigen Ring aus vier Silberionen und vier $N, N^{\prime}$-Pyrazol-Brückenliganden mit kristallographischer $\mathrm{S}_{4}$-Symmetrie. Zyklische $(\mathrm{PzAg})_{n}$-Kerne sind, wie schon erwähnt, ein häufig auftretendes Strukturmotiv in Münzmetall-Pyrazolat Komplexen [80-83], und ein ähnlicher tetranuklearer $(\mathrm{PzAg})_{n}$-Ring wurde zuvor schon in dem Silber(I)-Komplex von 3,5-Ditert-butylpyrazolat [84] und in D [79] beobachtet. Wie bei dem NMR-Spektrum von $\left[\left(\mathrm{H}_{2} \mathrm{~L}^{1}\right)_{4} \mathrm{Ag}_{4}\right]\left(\mathrm{PF}_{6}\right)_{8}$ festgestellt wurde, sind die Silberionen lediglich an die Pyrazolat-N-Atome gebunden während alle Seitenarm-Imidazoliumgruppen protoniert und frei sind. Das Imidazolium- $\mathrm{C}^{2} \mathrm{H}$ zeigt entweder schwache Wasserstoffbrückenbindung oder starke Wechselwirkungen zu den Sauerstoffatomen der benachbarten Lösungsmittelmoleküle (Aceton) oder $\mathrm{zu}$ den $\mathrm{PF}_{6}^{-}$-Anionen. Die vier Silberatome sind nahezu quadratisch angeordnet mit Ag...Ag Abständen von 3.1 . Die N-Ag-N Winkel $\left(168^{\circ}\right)$ weichen mehr von $180^{\circ}$ ab als die C-Ag-N Winkel in den $\left[\left(\mathrm{L}^{\mathrm{x}}\right)_{2} \mathrm{Ag}_{4}\right]^{2+}$-Komplexen.

Die Molekülstruktur des $\left[\left(\mathrm{H}_{2} \mathrm{~L}^{1}\right)_{4} \mathrm{Ag}_{4}\right]^{8+}$ hat große Ähnlichkeit zu dem kürzlich publizierten doppelkronenartigen $\mathrm{Ag}_{8}$-Komplex D, welcher den gleichen zentralen $(\mathrm{PzAg})_{4}$-Kern besitzt jedoch vier zusätzliche Silberionen in den peripheren Bis(NHC)-Einheiten aufweist. Der doppelkronenartige Komplex des Typs D wurde aus dem Ligandprecursor 3,5-Bis[3-(2,6-diisopropylphenyl)imidazolium-1-ylmethyl]$1 H$-pyrazol erhalten. [79] Es liegt demnach nah anzunehmen, dass eine weitere Reaktion des $\left[\left(\mathrm{H}_{2} \mathrm{~L}^{1}\right)_{4} \mathrm{Ag}_{4}\right]^{8+}$-Kations mit Silber(I)oxid durch Einbau zusätzlicher Silber(I)-Ionen in der äußeren Ligandensphäre zu Systemen des Typs D führen könnte, möglicherweise gefolgt von einer Neuanordnung, die das strukturelle Isomer $\left[\left(\mathrm{L}^{1}\right)_{2} \mathrm{Ag}_{4}\right]^{2+}$ (Typ $\mathrm{C}$ ) ergeben würde.

Die Bildung von $\left[\left(\mathrm{H}_{2} \mathrm{~L}^{1}\right)_{4} \mathrm{Ag}_{4}\right]^{8+}$ oder $\left[\left(\mathrm{L}^{1}\right)_{2} \mathrm{Ag}_{4}\right]^{2+}$ scheint lösungsmittelabhängig zu sein, da längeres Rühren einer Mischung des Ligandenprecursors $\left[\mathrm{H}_{3} \mathrm{~L}^{1}\right]\left(\mathrm{PF}_{6}\right)_{2}$ 


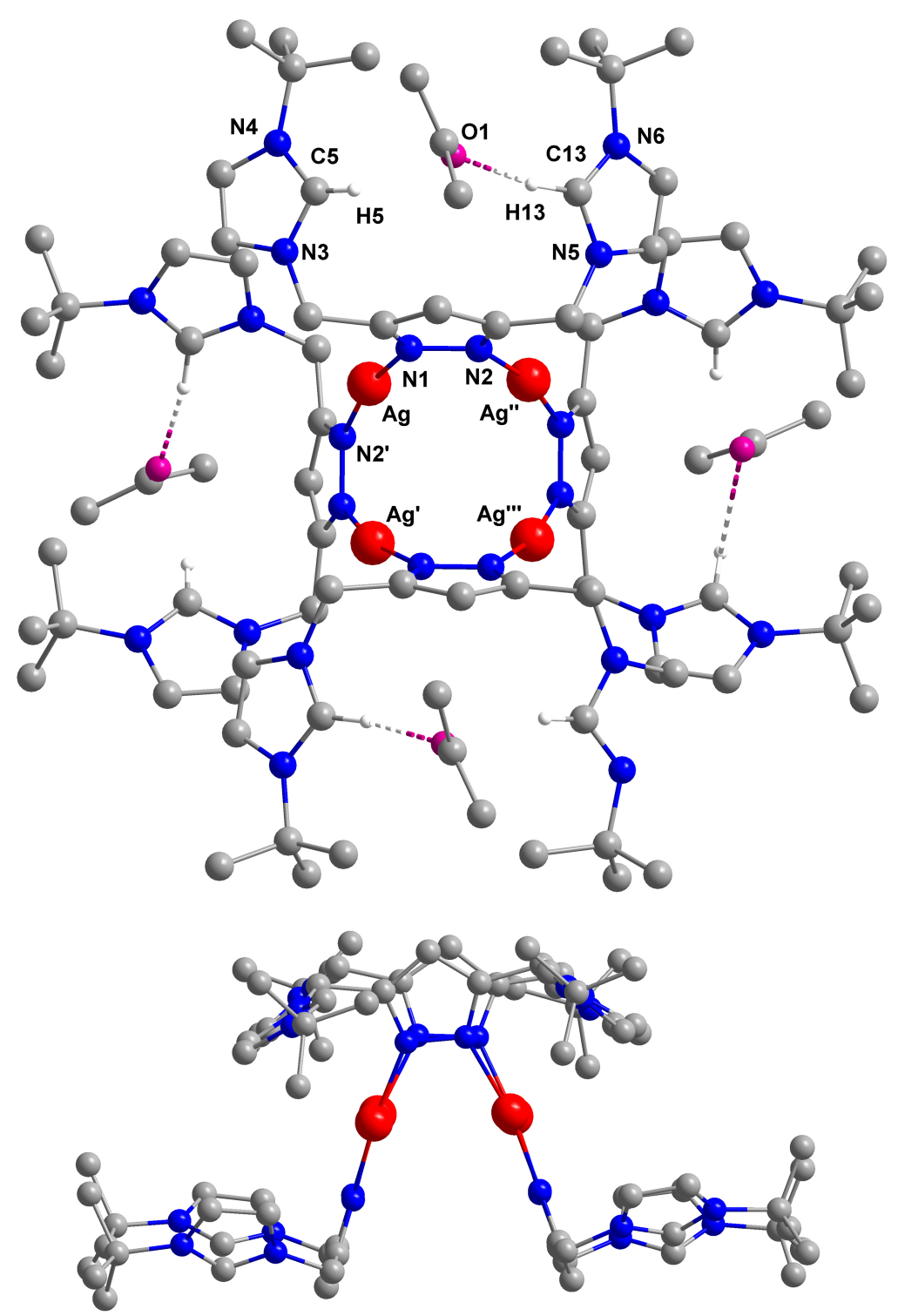

Abbildung 4.6: Festkörperstruktur von $\left[\left(\mathrm{H}_{2} \mathrm{~L}^{1}\right)_{4} \mathrm{Ag}_{4}\right]\left(\mathrm{PF}_{6}\right)_{8}$ (oben) und in einer seitlichen Ansicht entlang der Metall-Metall-Achse (unten). Aus Gründen der Übersichtlichkeit sind die nicht relevanten Wasserstoffatome sowie die Gegenionen nicht dargestellt. Symmetrieoperationen für äquivalente Atome: (') y, $1.5-\mathrm{x}, 1.5-\mathrm{z}$; (') $1.5-\mathrm{y}, \mathrm{x}, 1.5-\mathrm{z}$; ('"') 1.5- x, $1.5-\mathrm{y}$, z. 
und $\mathrm{Ag}_{2} \mathrm{O}$ in Aceton lediglich den ersten Komplex mit einem Ligand-zu-Metall Verhältnis von 1:1 ergibt, selbst bei Überschuss von $\operatorname{Ag}_{2} \mathrm{O}$. Im Unterschied dazu ergab die Behandlung von $\left[\left(\mathrm{H}_{2} \mathrm{~L}^{1}\right)_{4} \mathrm{Ag}_{4}\right]^{8+}$ mit zusätzlichen $2.2 \mathrm{Aq} \mathrm{Ag}_{2} \mathrm{O}$ für $48 \mathrm{~h}$ in Acetonitril eine Veränderung des ${ }^{1} \mathrm{H}-\mathrm{NMR}$ Spektrums (insbesondere war das Verschwinden des $\mathrm{CH}^{\text {Im2 }}$-Signals bei $8.56 \mathrm{ppm}$ zu beobachten). Diese Veränderungen deuten auf eine allmähliche Umwandlung zu $\left[\left(\mathrm{L}^{1}\right)_{2} \mathrm{Ag}_{4}\right]^{2+}$ hin mit einem Ligandzu-Metall Verhältnis von 1:2 im Endprodukt (Schema 4.3). In diesem Fall konnte kein Intermediat des Typs D nachgewiesen werden, was darauf hindeutet, dass die Verteilung der Spezies auch von den Imidazoliumsubstituenten abhängig ist. $\left[\left(\mathrm{L}^{1}\right)_{2} \mathrm{Ag}_{4}\right]^{2+}$ ist in DMSO- $d_{6}$ bei Raumtemperatur für mindestens einen Monat stabil. Es wurde keine besondere Lichtempfindlichkeit beobachtet.

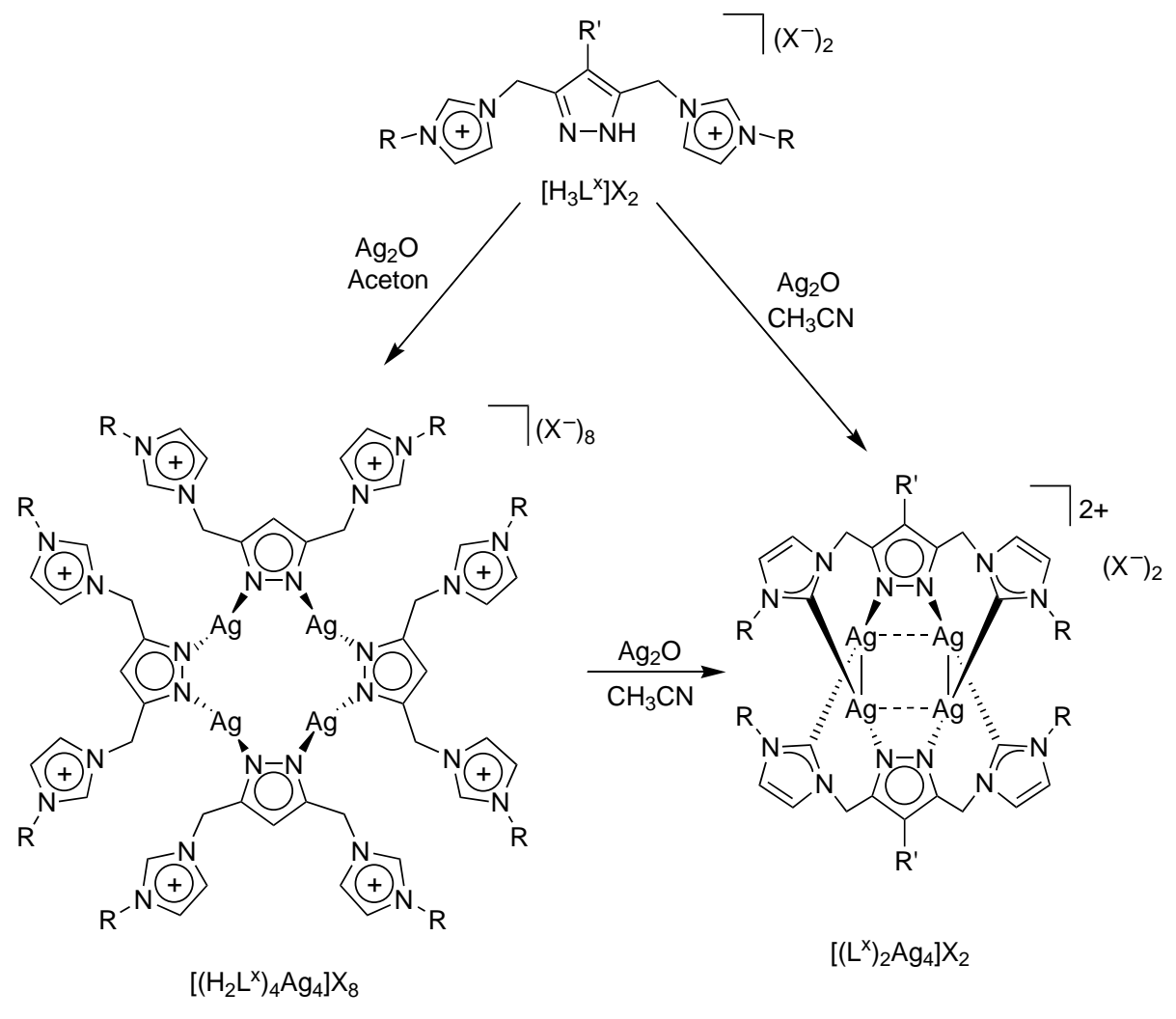

Schema 4.3: Synthese der Ag(I)-NHC-Komplexe.

In Tabelle 4.5 sind einige charakteristische Bindungslängen und -winkel von $\left[\left(\mathrm{L}^{1}\right)_{2} \mathrm{Ag}_{4}\right]\left(\mathrm{BF}_{4}\right)_{2},\left[\left(\mathrm{~L}^{3}\right)_{2} \mathrm{Ag}_{4}\right]\left(\mathrm{BF}_{4}\right)_{2},\left[\left(\mathrm{~L}^{4}\right)_{2} \mathrm{Ag}_{4}\right]\left(\mathrm{PF}_{6}\right)_{2}$ und $\left[\left(\mathrm{H}_{2} \mathrm{~L}^{1}\right)_{4} \mathrm{Ag}_{4}\right]\left(\mathrm{PF}_{6}\right)_{8} \mathrm{zu}-^{-}$ sammengefasst. 


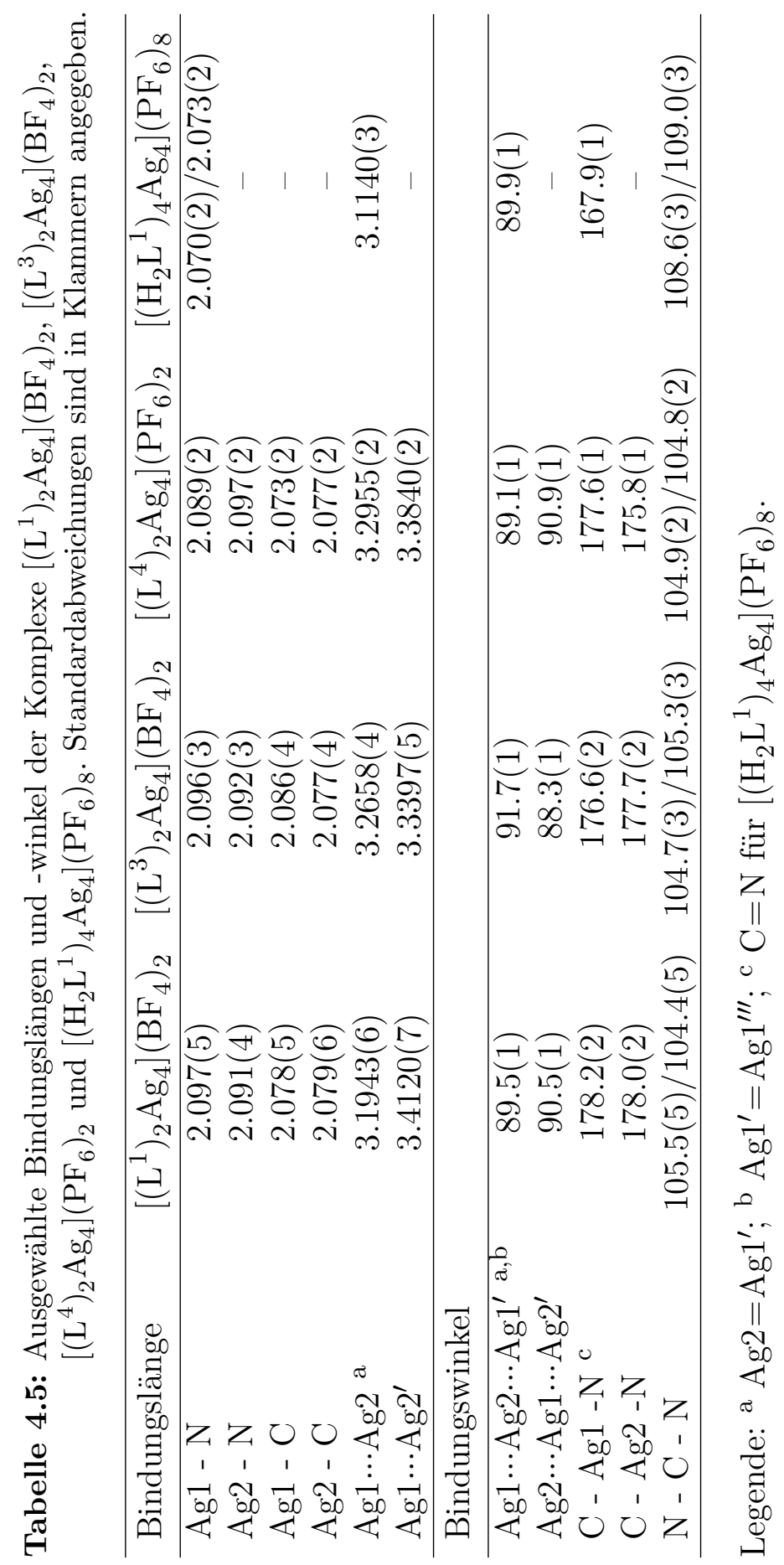


Des Weiteren konnte die Festkörperstruktur von $\left[\left(\mathrm{L}^{10}\right)_{2} \mathrm{Ag}_{4}\right]\left(\mathrm{BF}_{4}\right)_{2}$ bestimmt werden (Abbildung 4.7), nachdem farblose Kristalle durch Diethylether-Diffusion in eine Acetonitrillösung des Rohproduktes erhalten wurden. Diese Struktur ist konsistent mit den bisher in dieser Arbeit beschriebenen Strukturmotiven für die Silberkomplexe der Zusammensetzung $\left[\left(\mathrm{L}^{\mathrm{x}}\right)_{2} \mathrm{Ag}_{4}\right]^{2+}$. Wurde für die Kristallisation Aceton verwendet, konnten ebenso farblose Kristalle erhalten werden. Die röntgenographische Untersuchung dieser Kristalle ergab jedoch eine unterschiedliche Molekülstruktur mit der Zusammensetzung $\left.\left[\left(\mathrm{L}^{10}\right)_{2} \mathrm{Ag}_{6} \text { (Aceton }\right)_{2}\right]\left(\mathrm{BF}_{4}\right)_{4}$, welche in Abbildung 4.8 dargestellt ist. In dieser Struktur sind sechs Silber-Atome enthalten. Vier SilberAtome sind je an einem Pyrazolat-N-Atom und einem Carben-Kohlenstoffatom gebunden und gehen zusätzlich eine Wechselwirkung, mit einem benachbarten SilberAtom ein. Die restlichen zwei Silber-Atome sind an zwei Carben-Kohlenstoffatome und zwei benachbarte Silber-Atome gebunden und die letzte Koordinationsstelle ist durch ein Lösungsmittelmolekül besetzt. Diese Silber-Atome weisen eine ungewöhnliche Bindungsumgebung auf, welche vereinzelt in der Literatur beschrieben ist. $[74,78,123]$

Die Wahl des Lösungsmittels entscheidet, welcher Komplex im Festkörper gebildet wird: $\left.\left[\left(\mathrm{L}^{10}\right)_{2} \mathrm{Ag}_{6} \text { (Aceton }\right)_{2}\right]^{4+}$ oder $\left[\left(\mathrm{L}^{10}\right)_{2} \mathrm{Ag}_{4}\right]^{2+}$. Das ESI-Massenspektrum von $\left[\left(\mathrm{L}^{10}\right)_{2} \mathrm{Ag}_{4}\right]\left(\mathrm{BF}_{4}\right)_{2}$ zeigt einen Peak bei einem $m / z$-Verhältnis von 1389a.u., welcher mit Hilfe des Isotopenmusters dem Fragment $\left[\mathrm{M}-\mathrm{BF}_{4}\right]^{+}$zugeordnet werden konnte. Der intensivste Peak wird bei einem $m / z$-Verhältnis von 651 a.u. beobachtet und gehört zum Fragment $\left[\mathrm{M}-2 \mathrm{BF}_{4}\right]^{2+}$. Das gleiche ESI-Massenspektrum wurde von dem sechskernigen Ag-Komplex $\left.\left[\left(\mathrm{L}^{10}\right)_{2} \mathrm{Ag}_{6} \text { (Aceton }\right)_{2}\right]\left(\mathrm{BF}_{4}\right)_{4}$ erhalten, irrelevant ob der Kristall in Aceton oder Acetonitril gemessen wurde. Auch durch NMR-spektroskopische Untersuchungen konnte gezeigt werden, dass in Lösung nur eine Spezies vorliegt. Die $\mathrm{CH}_{3}$-Gruppen spalten zu Dubletts aufgrund der Chiralität auf. Für das $\mathrm{C}^{4}$-Proton am Pyrazol ist ein Singulett bei einer Verschiebung von 6.2-6.4ppm zu sehen. Es gibt zwei Signale für die Protonen der verbrückenden Methylengruppen, welche als charakteristisches AB-System erscheinen und zwei Signale für die Seitenarm-CH-Protonen, die hier auch wie erwartet zum Quartett aufspalten. Die ${ }^{109} \mathrm{Ag}$-Resonanz wird durch die Kopplung mit Pyrazol-, Imidazolund $\mathrm{CH}_{2}$-Protonen detektiert und erscheint für $\left.\left[\left(\mathrm{L}^{10}\right)_{2} \mathrm{Ag}_{6} \text { (Aceton }\right)_{2}\right]\left(\mathrm{BF}_{4}\right)_{4}$ und $\left[\left(\mathrm{L}^{10}\right)_{2} \mathrm{Ag}_{4}\right]\left(\mathrm{BF}_{4}\right)_{2}$ bei 637 bzw. 638ppm. Die chemischen Verschiebungen und Kopplungskonstanten stimmen mit denen zuvor in dieser Arbeit und in der Literatur untersuchten Pyrazolat-NHC Komplexen überein. [35]

Sämtliche Versuche solche sechskernigen Silber-Verbindungen gezielt zu synthetisieren blieben erfolglos. Ausgehend vom Liganden führte die sukzessive Zugabe 


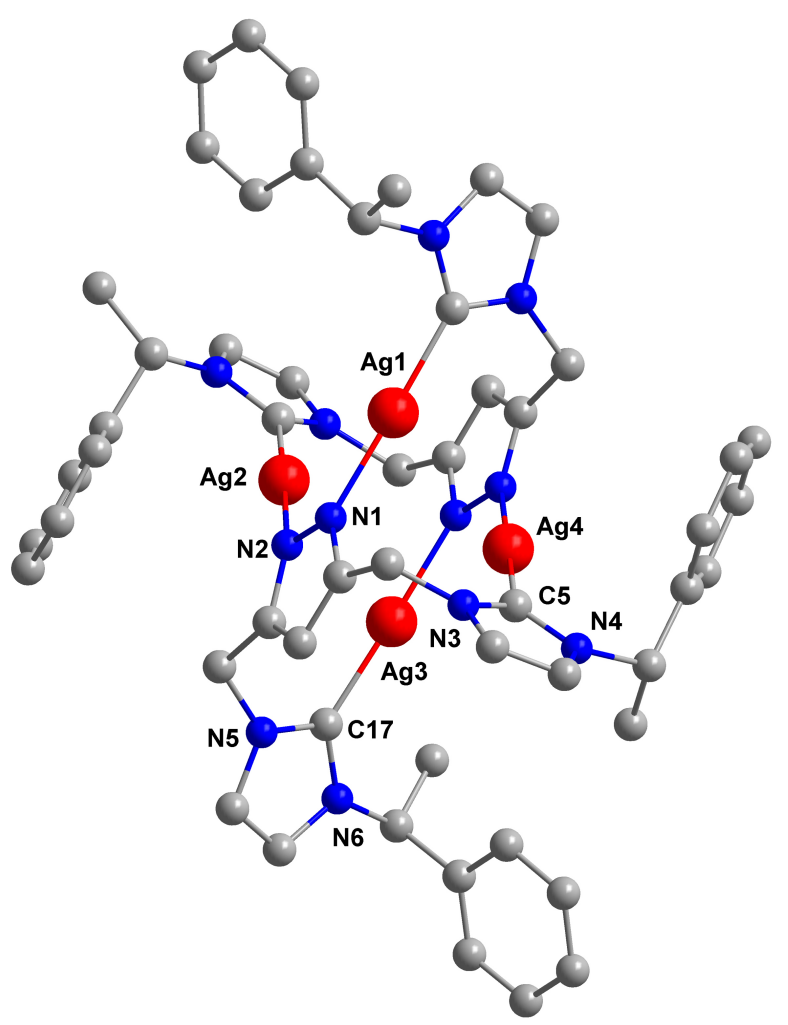

Abbildung 4.7: Festkörperstruktur von $\left[\left(\mathrm{L}^{10}\right)_{2} \mathrm{Ag}_{4}\right]\left(\mathrm{BF}_{4}\right)_{2}$. Aus Gründen der Übersichtlichkeit sind die Wasserstoffatome und die $\mathrm{BF}_{4}^{-}$-Ionen nicht dargestellt.

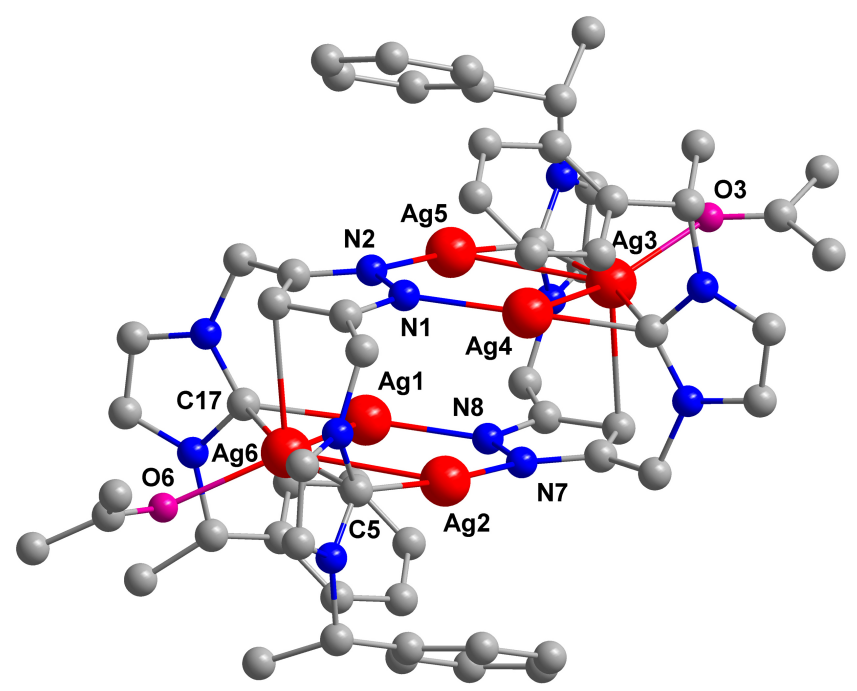

Abbildung 4.8: Festkörperstruktur von $\left[\left(\mathrm{L}^{10}\right)_{2} \mathrm{Ag}_{6}(\text { Aceton })_{2}\right]\left(\mathrm{BF}_{4}\right)_{4}$. Aus Gründen der Übersichtlichkeit sind Wasserstoffatome und Gegenionen nicht dargestellt. 
von $\mathrm{Ag}_{2} \mathrm{O}$ und teilweise von $\mathrm{AgBF}_{4}$ nicht zum gewünschten sechskernigen SilberKomplex. Daraufhin wurde untersucht, ob eine Umwandlung vom bestehenden Komplex $\left[\left(\mathrm{L}^{10}\right)_{2} \mathrm{Ag}_{4}\right]^{2+}$ zum sechskernigen Komplex $\left[\left(\mathrm{L}^{10}\right)_{2} \mathrm{Ag}_{6}(\text { Aceton })_{2}\right]^{4+}$ möglich ist. Hierzu wurde eine Lösung von $\left[\left(\mathrm{L}^{10}\right)_{2} \mathrm{Ag}_{4}\right]^{2+}$ in Aceton mit zusätzlichen $\mathrm{AgBF}_{4}$ versetzt. NMR-spektroskopische Untersuchungen zeigen keine Veränderungen im ${ }^{1} \mathrm{H}-\mathrm{NMR}$ Spektrum. Für diese Zwecke wurde die 1-Benzyl-Gruppe im Seitenarm eingeführt, da eine mögliche Abschirmung der Silber-Atome von den Phenylgruppen vermutet wurde. Die Synthese des $\left[\left(\mathrm{L}^{8}\right)_{2} \mathrm{Ag}_{4}\right](\mathrm{PF})_{6}$-Komplexes konnte spektroskopisch bestätigt werden, die eines sechskernigen Komplexes aber nicht.

Abschließend kann also festgestellt werden, dass die Bildung von $\left[\left(\mathrm{L}^{\mathrm{x}}\right)_{2} \mathrm{Ag}_{4}\right]^{2+}$ oder dem neuartigen sechskernigen Silber-Komplex im Fall der chiralen 1-PhenylethylSeitenarme eine Eigenschaft ist, die nur im Festkörperzustand auftritt, indem Lösungsmittelmoleküle sich mit zusätzlichen Silber-Atomen in der Struktur anlagern. Es wurde kein dynamisches Verhalten in Lösung zwischen den beiden Strukturen beobachtet.

Zusätzlich wurde aus einem analogen Kristallisationsansatz von $\left[\left(\mathrm{L}^{10}\right)_{2} \mathrm{Ag}_{4}\right]\left(\mathrm{BF}_{4}\right)_{2}$ in einer Acetonitrillösung das $\left[(\mathrm{MeCN})_{4} \mathrm{Ag}\right](\mathrm{BF})_{4}$-Addukt kristallisiert, welches des öfteren als Nebenprodukt bei der Synthese bzw. Kristallisation ähnlicher Silberkomplexe auftritt. [124] Weitere Kristallstrukturdaten der Silber(I)-NHC-Komplexe sind in Kapitel 7 zusammengefasst.

Die Silber-NHC-Komplexe zeigen außerdem emittierende Eigenschaften. Absorptions- und Emissionsspektren wurden von frisch angesetzten Lösungen der Komplexe aufgenommen. Ein interessantes Absorptions- bzw. Emissionsspektrum ist in Abbildung 4.9 dargestellt. Die Spektren dieses neuartigen tetranuklearen Silber(I)-NHC-Komplexes $\left(\left[\left(\mathrm{H}_{2} \mathrm{~L}^{1}\right)_{4} \mathrm{Ag}_{4}\right]\left(\mathrm{PF}_{6}\right)_{8}\right)$ in Acetonitril zeigen fluoreszierende Eigenschaften in Lösung. Im UV/Vis Spektrum ist eine starke Absorption bei $240 \mathrm{~nm}$ mit einer Schulter bei etwa $255 \mathrm{~nm}$ zu beobachten. Aus dem Emissionsspektrum geht eine breite, bei etwa $310 \mathrm{~nm}$ zentrierte Bande hervor, wenn bei $\lambda_{e x}=265 \mathrm{~nm}$ angeregt wird.

Anders als bei bereits bekannten tetranuklearen Silber-Komplexen [34] konnten bei Raumtemperatur von Verbindungen $\left[\left(\mathrm{L}^{1}\right)_{2} \mathrm{Ag}_{4}\right]\left(\mathrm{BF}_{4}\right)_{2}$ und $\left[\left(\mathrm{L}^{9}\right)_{2} \mathrm{Ag}_{4}\right]\left(\mathrm{BF}_{4}\right)_{2}$ keine Absorptionsbanden beobachtet werden. 
Tabelle 4.6: Ausgewählte Bindungslängen und -winkel von $\left[\left(\mathrm{L}^{10}\right)_{2} \mathrm{Ag}_{4}\right]\left(\mathrm{BF}_{4}\right)_{2}$ und $\left[\left(\mathrm{L}^{10}\right)_{2} \mathrm{Ag}_{6}(\text { Aceton })_{2}\right]\left(\mathrm{BF}_{4}\right)_{4}$. Standardabweichungen sind in Klammern angegeben.

\begin{tabular}{|c|c|c|}
\hline$\left[\left(\mathrm{L}^{10}\right)_{2} \mathrm{Ag}_{4}\right]\left(\mathrm{BF}_{4}\right)_{2}$ & Bindungslänge $[\AA]$ & Winkel $\left[{ }^{\circ}\right]$ \\
\hline Ag1 - N1 & $2.087(5)$ & \\
\hline Ag1 - Ag2 & $3.3262(5)$ & \\
\hline $\operatorname{Ag} 1-\operatorname{Ag} 4$ & $3.0484(5)$ & \\
\hline C44 - Ag1 - N1 & & $178.40(18)$ \\
\hline $\mathrm{C} 44-\mathrm{Ag} 1-\mathrm{Ag} 4$ & & $108.03(14)$ \\
\hline N1 - Ag1 - Ag4 & & $72.76(11)$ \\
\hline$\left[\left(\mathrm{L}^{10}\right)_{2} \mathrm{Ag}_{6}(\text { Aceton })_{2}\right]\left(\mathrm{BF}_{4}\right)_{4}$ & & \\
\hline Ag1 - Ag6 & $2.7748(6)$ & \\
\hline Ag2 - Ag6 & $2.7848(6)$ & \\
\hline Ga1 - Ag5 & $3.2024(5)$ & \\
\hline Ag6 - O6 & $2.296(3)$ & \\
\hline Ag1 - N8 & $2.098(4)$ & \\
\hline Ag1 - C17 & $2.108(6)$ & \\
\hline Ag2 - C5 & $2.145(5)$ & \\
\hline N8 - Ag1 - C17 & & $175.11(18)$ \\
\hline N8 - Ag1 - Ag6 & & $117.16(11)$ \\
\hline C17 - Ag1 - Ag6 & & $57.96(14)$ \\
\hline Ag6 - Ag1 - Ag5 & & $110.240(17)$ \\
\hline C5 - Ag2 - N7 & & $171.78(17)$ \\
\hline
\end{tabular}

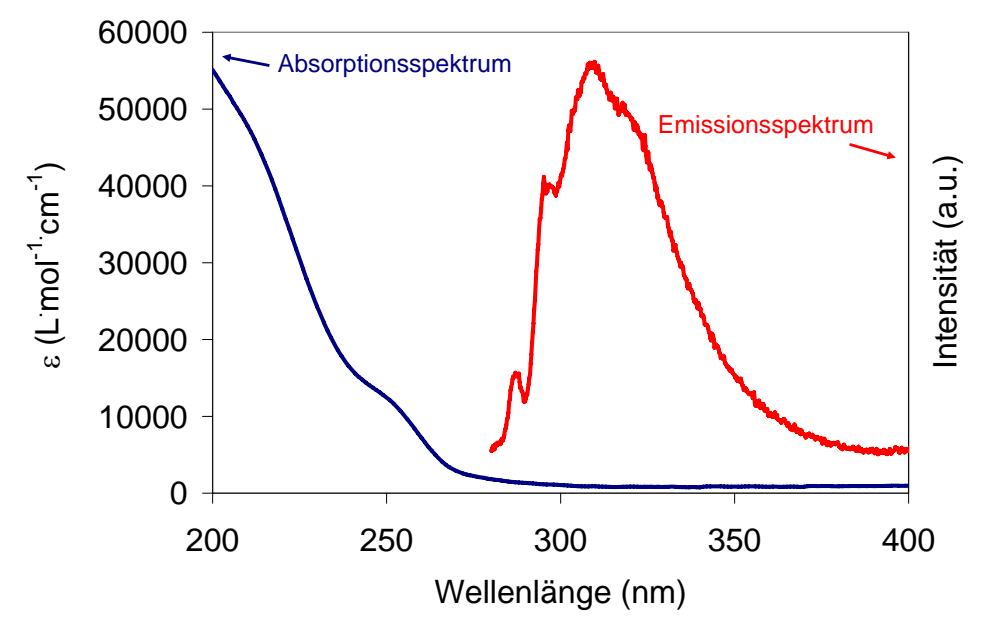

Abbildung 4.9: Absorptions- und normalisiertes Emissionsspektrum $\left(\lambda_{e x}=265 \mathrm{~nm}\right)$ von $\left[\left(\mathrm{H}_{2} \mathrm{~L}^{1}\right)_{4} \mathrm{Ag}_{4}\right]\left(\mathrm{PF}_{6}\right)_{8}$ aufgenommen bei RT in MeCN-Lösung. 


\subsubsection{Palladium-NHC-Komplexe}

Wie in Kapitel 2.2 gezeigt wurde, gibt es einige Möglichkeiten zur Herstellung von NHC-Metallkomplexen. Vielversprechend sind besonders diese zwei Möglichkeiten:

a) Umsetzung der Liganden, nach in situ Deprotonierung, mit dem entsprechenden Metallsalz

b) Transmetallierung von Silber(I)-NHC-Komplexen

Betrachtet man zuerst Möglichkeit a), so wird der entsprechende Ligand direkt mittels einer externen Base deprotoniert. Anschließend wird der MetallsalzVorläufer dazugegeben. Schema 4.4 zeigt die Umsetzung eines pyrazolatverbrückten Bis(imidazolium)-Liganden mit Kalium-bis-(trimethylsilyl)amid $\left(\mathrm{KN}\left(\mathrm{SiMe}_{3}\right)_{2}\right)$. Nach Zugabe des Metallvorläufers Allylpalladium(II)-chlorid Dimer wird der zweikernige Palladium-NHC-Komplex erhalten.

Je nachdem, ob der Ligand als Hydrochlorid eingesetzt wird oder nicht, ist zusätzlich die Zugabe von $\mathrm{AgBF}_{4}$ erforderlich, um das Gegenion des Komplexes in situ auszutauschen. Die Synthesen wurden mit dem Allylpalladium(II)-chlorid Dimer in stöchiometrischen Menge durchgeführt.

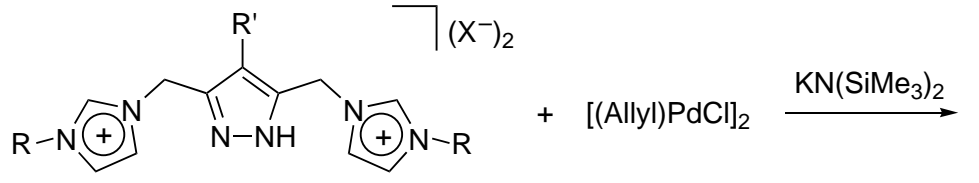

$\left[\mathrm{H}_{3} \mathrm{~L}^{\mathrm{X}}\right] \mathrm{X}_{2} ; \mathrm{X}=\mathrm{BF}_{4}, \mathrm{PF}_{6}$

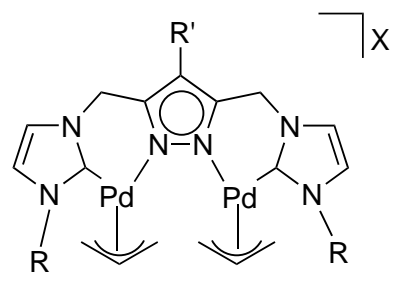

Schema 4.4: Synthese von Palladium-NHC-Komplexen mittels Deprotonierung mit externer Base.

Durch Anwendung der Synthesestrategie b), welche über die Transmetallierung der Silber(I)-NHC-Komplexe verläuft, findet eine Übertragung eines NHCs auf ein anderes Metall statt; diese Route ist aufgrund ihrer Einfachheit vielfältig einsetzbar. Silber(I)-NHC-Komplexe sind für die Übertragung auf viele Übergansmetalle (Rh, $\mathrm{Ir}, \mathrm{Ru}$ und $\mathrm{Cu}$ ) besonders gut geeignet, vor allem aber auf Palladium. Somit konnte nach dieser Methode im Rahmen dieser Arbeit eine Reihe von NHC-Komplexe synthetisiert werden (Schema 4.5).

Zu den Vorteilen gegenüber der in situ Deprotonierungs-Synthesemethode (a) zählt die einfache Durchführung; der apparative Aufwand ist sehr gering, da die Reaktionen nicht unter Inertbedingungen stattfinden müssen. Die Abtrennung von anorganischen Nebenprodukten erweist sich als äußerst unkompliziet - das bei der Reaktion 


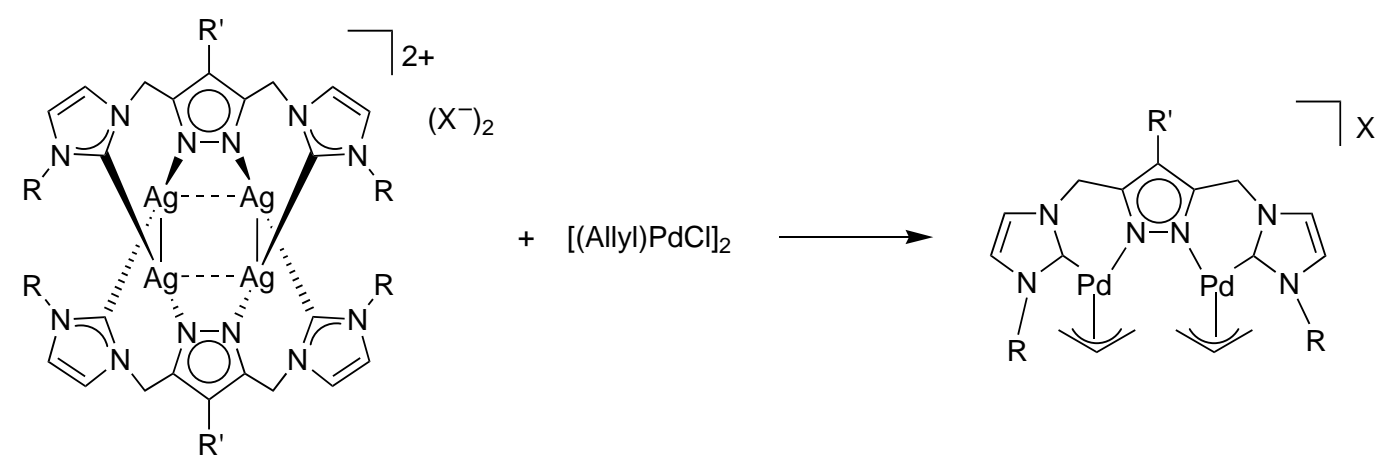

$\left[\left(\mathrm{L}^{\mathrm{X}}\right)_{2} \mathrm{Ag}_{4}\right] \mathrm{X}_{2} ; \mathrm{X}=\mathrm{BF}_{4}, \mathrm{PF}_{6}$

Schema 4.5: Synthese von Palladium-NHC-Komplexen unter Transmetallierung von Silber-NHC-Komplexen.

entstandene $\mathrm{AgCl}$ muss lediglich abfiltriert werden. Die resultierenden PalladiumNHC-Komplexe sind in guten, nahezu quantitativen Ausbeuten darstellbar. Der Syntheseweg der in situ Deprotonierung bringt gegenüber dem des Ligandentransfers von Silber(I)-NHC-Komplexen auf Palladium, im Bezug auf die Ausbeuten, keine signifikanten Vorteile und wurde für die nachfolgenden Umsetzungen nicht weiter verfolgt.

Zuerst erfolgten einige Komplexierungen an Palladium(II). Als Palladiumsalz wurde Allylpalladiumchlorid verwendet. Sämtliche dargestellten Ag-Komplexe konnten zu Allylpalladiumkomplexen gemäß Schema 4.5 ummetalliert werden. Die gewählten Reaktionsbedingungen sind erprobt [35] und erfolgen in sehr guten Ausbeuten. Es wurde in allen Fällen ein Palladium-zu-Ligand Verhältnis von 2:1 eingehalten, da der Zugang zu bimetallischen Komplexen erreicht werden soll.

Es gelang, einige der synthetisierten Palladiumkomplexe zu kristallisieren. Kristalle von $\left[\mathrm{L}^{5} \mathrm{Pd}_{2}(\mathrm{Allyl})_{2}\right] \mathrm{PF}_{6}$ und $\left[\mathrm{L}^{1} \mathrm{Pd}_{2}(\mathrm{Allyl})_{2}\right] \mathrm{BF}_{4}$ konnten durch langsame Diethylether-Diffusion in eine Acetonitrillösung des jeweilligen Komplexes erhalten werden. Es entstanden farblose Kristalle, die sich zur Röntgenkristallstrukturanalyse eigneten. Die Festkörperstruktur von $\left[\mathrm{L}^{5} \mathrm{Pd}_{2}(\mathrm{Allyl})_{2}\right] \mathrm{PF}_{6}$ ist in Abbildung 4.10 dargestellt. Kristallstrukturdaten dieser Verbindung sind in Kapitel 7 zusammengefasst. In Tabelle 4.7 sind charakteristische Bindungslängen und -winkel angegeben.

Die Molekülstruktur von $\left[\mathrm{L}^{5} \mathrm{Pd}_{2}(\mathrm{Allyl})_{2}\right] \mathrm{PF}_{6}$ setzt sich aus einem Pyrazolatliganden und zwei verbrückten Palladiumionen zusammen. Die Palladiumionen sind von je einem NHC und einem N(Pyrazolat) des zentralen Pyrazolatliganden koordiniert. Jedes Palladiumatom ist zusätzlich von einem Allylliganden umgeben. Die Koordinationsgeometrie um jedes Palladium ist verzerrt quadratisch-planar. Die 


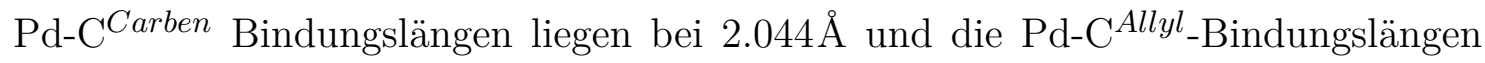
zwischen 2.097 und 2.266 $\AA$. Der Abstand zwischen den zwei Palladiumatomen beträgt $4.066 \AA$. Dieser Pd...Pd-Abstand unterscheidet sich jedoch nicht wesentlich von den Abständen weiterer bekannter zweikerniger Palladium(II)-Allyl-Komplexe mit verbrückenden Pyrazolatliganden. Alle weitere Werte stimmen genauso mit Daten aus Literaturangaben gut überein. [35]

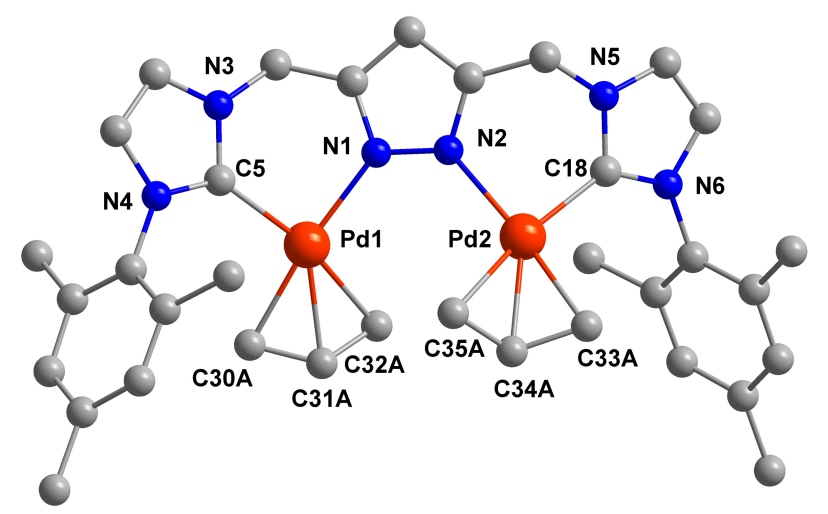

Abbildung 4.10: Festkörperstruktur von $\left[\mathrm{L}^{5} \mathrm{Pd}_{2}(\mathrm{Allyl})_{2}\right] \mathrm{PF}_{6}$. Aus Gründen der Übersichtlichkeit sind die Wasserstoffatome, das Gegenion und das Lösungsmittelmolekül nicht dargestellt.

Tabelle 4.7: Ausgewählte Bindungslängen und -winkel von $\left[\mathrm{L}^{5} \mathrm{Pd}_{2}(\mathrm{Allyl})_{2}\right] \mathrm{PF}_{6}$. Standardabweichungen sind in Klammern angegeben.

\begin{tabular}{l|cc}
\hline$\left[\mathrm{L}^{5} \mathrm{Pd}_{2}(\text { Allyl })_{2}\right] \mathrm{PF}_{6}$ & Bindungslänge $[\AA]$ & Winkel $\left[^{\circ}\right]$ \\
\hline $\mathrm{Pd} 1-\mathrm{N} 1$ & $2.094(3)$ & \\
$\mathrm{Pd} 1-\mathrm{C} 5$ & $2.044(4)$ & \\
$\mathrm{Pd} 1-\mathrm{C} 30$ & $2.124(8)$ & \\
$\mathrm{Pd} 1-\mathrm{C} 31$ & $2.162(5)$ & \\
$\mathrm{Pd} 1-\mathrm{C} 32$ & $2.266(13)$ & \\
$\mathrm{Pd} 2-\mathrm{C} 33$ & $2.097(7)$ & \\
$\mathrm{Pd} 2-\mathrm{C} 34$ & $2.147(6)$ & \\
$\mathrm{Pd} 2-\mathrm{C} 35$ & $2.190(7)$ & \\
$\mathrm{Pd} 1 \cdots \mathrm{Pd} 2$ & $4.0662(4)$ & \\
$\mathrm{C} 5-\mathrm{Pd} 1-\mathrm{N} 1$ & & $87.63(13)$ \\
$\mathrm{C} 5-\mathrm{Pd} 1-\mathrm{C} 30$ & & $100.3(2)$ \\
$\mathrm{N} 1-\mathrm{Pd} 1-\mathrm{C} 30$ & & $171.9(2)$ \\
\hline
\end{tabular}

Die Struktur des Palladiumkomplexes konnte zusätzlich mit Hilfe NMRspektroskopischer Methoden bestätigt werden. Die Zuordnung der chemischen Verschiebungen der zugehörigen Wasserstoff- und Kohlenstoffatome gelang mit Hilfe 
von CH-COSY Experimenten. Charakteristisch sind im ${ }^{13} \mathrm{C}-\mathrm{NMR}$ Spektrum die Signale bei etwa 47, 69 und 116ppm. Diese Signale gehören zu den Allylgruppen und deuten auf die Bildung des Allylcarbenpalladiumkomplexes hin. Ein weiterer Hinweis auf eine erfolgreiche Umsetzung zum Allylkomplex ist die Verschiebung des $\mathrm{C}^{\mathrm{Im} 2}$-Signals bei $176 \mathrm{ppm}$, welche, im Vergleich zu der chemischen Verschiebung von etwa 118-122 ppm im entsprechenden Silberkomplex, deutlich tieffeldverschoben ist. Ein Vergleich mit Literaturwerten zeigt, dass die gefundenen chemischen Verschiebungen im Erwartungsbereich liegen. [35] Im ${ }^{1} \mathrm{H}-\mathrm{NMR}$ Spektrum sind hingegen keine deutlichen Verschiebungen sowohl der Allyl- als auch der Carben-Signale zu beobachten. Außerdem wurde der Palladiumkomplex durch Massenspektrometrie charakterisiert.

Die Allylliganden des bimetallischen Komplexes $\left[\mathrm{L}^{5} \mathrm{Pd}_{2}(\mathrm{Allyl})_{2}\right] \mathrm{PF}_{6}$ sind fehlgeordnet. Es existieren zwei isomere Formen mit unterschiedlichen Orientierungen der Allylgruppen (Schema 4.7). Um herauszufinden, ob ein dynamisches Gleichgewicht in Lösung zwischen den beiden Isomeren herrscht, wurden detaillierte NMR-spektroskopische Untersuchungen vorgenommen. ${ }^{1} \mathrm{H}$-NMR Spektren von $\left[\mathrm{L}^{5} \mathrm{Pd}_{2}(\mathrm{Allyl})_{2}\right] \mathrm{PF}_{6}$ sind in Acetonitril und unterschiedlichen Temperaturen aufgenommen worden. Jedes der Isomere zeigt fünf eindeutige Resonanzen für die beiden Allylliganden. Beachtlich ist die Beobachtung, dass das Signal für die verbrückenden $\mathrm{CH}^{2}$-Gruppen für das eine Isomer als AB Muster und für das andere als scharfes Singulett erscheint. Bei Raumtemperatur kommen diese isomeren Formen in gleichen Mengen vor, was im Temperaturbereich von 25 bis $50^{\circ} \mathrm{C}$ nahezu weiterhin so bleibt (Verhältnis 1:1.1). Erst bei tieferer Temperatur $\left(-40^{\circ} \mathrm{C}\right)$ kommt es zu einer Verkleinerung und Verbreiterung aller Allyl-Signale des eines Isomers.

Aus dem ${ }^{1} \mathrm{H}$-EXSY Spektrum von $\left[\mathrm{L}^{5} \mathrm{Pd}_{2}(\text { Allyl })_{2}\right] \mathrm{PF}_{6}$, aufgenommen mit einer Mischzeit von 500ms, ergibt sich die Rotation eines der Allylliganden. Demnach geht das eine Isomer in das andere über $\left(k_{h i n}=0.20 s^{-1} ; k_{r u ̈ c k}=0.22 s^{-1}\right)$. Eine Zuordnung der Allylresonanzen konnte durch NOE-Korrelation erfolgen. Diese Zuordnung stimmt mit Werten aus der Literatur überein. [35] Es wird angenommen, dass die gefundene Allylrotation via einer $\eta^{1}$-Zwischenstufe verfolgt. Hierbei wird die freie Koordinationsstelle zwischenzeitlich von einem Lösungsmittelmolekül besetzt. [125] Der Mechanismus verläuft nach einem syn/anti-Austausch (Schema 4.6). Die äußeren allylischen Kohlenstoff-Atome tauschen mitenander nicht aus. Es ändert sich lediglich die Geometrie des mittleren $\mathrm{C}^{2}$-Kohlenstoffatoms. Die Selektivität dieses Prozesses kann durch den trans-Effekt oder auch sterische Faktoren beeinflusst werden. Ein starker trans-Effekt des NHC-Donors sorgt für eine Schwächung der Bindung zwischen der $\mathrm{CH}_{2}$-Gruppe in trans-Stellung und dem Carben. Die $\mathrm{CH}_{2}$-Gruppe in trans-Stellung ist deshalb aktiver als die in cis-Stellung. Bei 
Verbindung $\left[\mathrm{L}^{5} \mathrm{Pd}_{2}(\mathrm{Allyl})_{2}\right] \mathrm{PF}_{6}$ bleibt aus diesem Grund die $\mathrm{CH}_{2}$-Gruppe in cisStellung im $\eta^{1}$-Intermediat am Pd-Zentrum gebunden und tauscht auf diese Weise zwei „entgegengesetzte“ Protonen aus. Durch die $\eta^{3}-\eta^{1}-\eta^{3}$-Isomerisierung verändert sich die relative Orientierung der beiden Allylliganden.

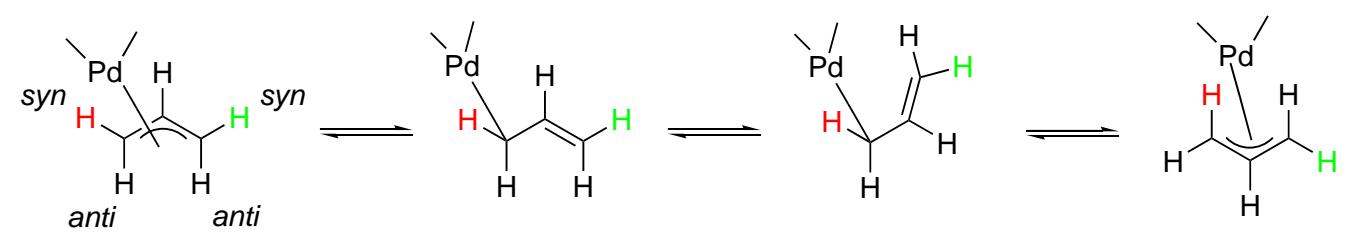

Schema 4.6: Mechanismus für eine $\eta^{3}-\eta^{1}-\eta^{3}$-Isomerisierung in einem Allylpalladiumkomplex.

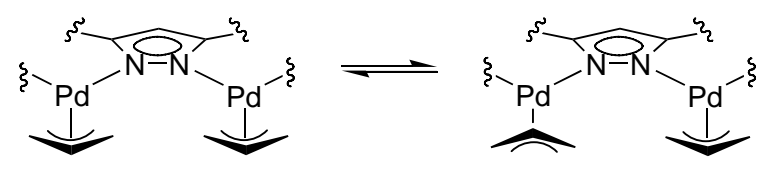

Schema 4.7: Unterschiedliche Orientierung der Allylgruppen in Palladium-NHCKomplexen mit der Zusammensetzung $\left[\mathrm{L}^{\mathrm{x}} \mathrm{Pd}_{2}(\mathrm{Allyl})_{2}\right]^{2+}$.

In Abbildung 4.11 ist die Festkörperstruktur von $\left[\mathrm{L}^{1} \mathrm{Pd}_{2}(\mathrm{Allyl})_{2}\right] \mathrm{BF}_{4}$ dargestellt. Die Bindungslängen und -winkel von $\left[\mathrm{L}^{1} \mathrm{Pd}_{2}(\mathrm{Allyl})_{2}\right] \mathrm{BF}_{4}$ sind vergleichbar denen von $\left[\mathrm{L}^{5} \mathrm{Pd}_{2}(\text { Allyl })_{2}\right] \mathrm{PF}_{6}$. Es konnten nur wenige Kristalle erhalten werden, die für die röntgenographische Analyse geeignet waren und alle hier präsentierten Ergebnisse resultieren aus dem Rohprodukt des Reaktionsgemisches. Die Verbindung wurde zusätzlich durch Massenspektrometrie charakterisiert. Das ESI-Massenspektrum zeigt den höchsten Peak bei einem $m / z$-Verhältnis von 635a.u., welcher mit Hilfe des Isotopenmusters zum Fragment $\left[\mathrm{M}-\mathrm{BF}_{4}\right]^{+}$zugeordnet werden konnte. Die Bildung dieses Komplexes wurde ebenso durch hochauflösende Massenspektrometrie mit einem Peak bei $m / z$-Verhältnis von 635.1161a.i. bestätigt. Bei Raumtemperatur war sowohl im ${ }^{1} \mathrm{H}-\mathrm{NMR}$ als auch im ${ }^{13} \mathrm{C}-\mathrm{NMR}$ Spektrum eine Verbreiterung der Signale im allylischen Bereich zu beobachten. Tieftemperatur NMR Messungen waren notwendig, um eine mögliche Zuordnung der Signale zu treffen. Die Zuordnung der Signale erfolgte durch die Aufnahme verschiedener 2D-Spektren (u.a. HH-COSY, NOESY und CH-COSY bzw. HSQC). Das interessante am ${ }^{1} \mathrm{H}-\mathrm{NMR}$ Spektrum, welches bei $-25^{\circ} \mathrm{C}$ aufgenommen wurde, war die Existenz dreier Signale (alle Singuletts) für das $\mathrm{C}^{4}$-Proton am Pyrazol. Diese gehören zu zwei symmetrisch aufgebauten Verbindungen (S1 und S2) und einer unsymmetrischen Verbindung (AS) mit einem Verhältnis von 2:1.05:0.6 (AS:S1:S2). Diese Verbindungen kommen da- 
durch zustande, dass einerseits die Allyl-Gruppen unterschiedliche Orientierungen relativ zueinander einnehmen können, wobei parallele und antiparallele Orientierungen über eine $\eta^{3}-\eta^{1}-\eta^{3}$-Allyl-Rotation miteinander im Gleichgewicht stehen. Diese Allyl-Rotation wurde wie bereits in der Literatur erwähnt [35] auch bei der sehr ähnlichen Verbindung $\left[\mathrm{L}^{5} \mathrm{Pd}_{2}(\mathrm{Allyl})_{2}\right] \mathrm{PF}_{6}$ in dieser Arbeit studiert und ist selbst in gut koordinierenden Lösungsmitteln (Acetonitril) relativ langsam $\left(<1 \mathrm{~s}^{-1}\right.$ bei Raumtemperatur). Gleichzeitig treten bei $\left[\mathrm{L}^{1} \mathrm{Pd}_{2}(\mathrm{Allyl})_{2}\right] \mathrm{BF}_{4}$ unterschiedliche Orientierungen der $t \mathrm{Bu}$-Gruppen relativ zu den Allyl-Gruppen auf: aus der ,antiparallelen“ Allyl-Konfiguration werden so zwei jeweils $C_{2}$ symmetrische Verbindungen, aus der „parallelen“ eine komplett unsymmetrische Verbindung. Das „Umklappen“ der $t$ BuGruppen führt bei $\left[\mathrm{L}^{1} \mathrm{Pd}_{2}(\text { Allyl })_{2}\right] \mathrm{BF}_{4}$ bei Raumtemperatur zur Signalverbreiterung und ist erst bei $-25^{\circ} \mathrm{C}$ genügend langsam $\left(<1 \mathrm{~s}^{-1}\right)$. Bei höherer Temperatur, ab etwa $25^{\circ} \mathrm{C}$, laufen die zwei Signale der symmetrischen Verbindungen zu einem breiten Signal zusammen. Das $\mathrm{C}^{4}$-Proton am Pyrazol in AS bleibt dagegen scharf. Eine ähnliche Beobachtung mit drei nebeneinander existierenden Verbindungen wurde ebenso bei den Komplexen $\left[\mathrm{L}^{4} \mathrm{Pd}_{2}(\mathrm{Allyl})_{2}\right]^{+}$und $\left[\mathrm{L}^{11} \mathrm{Pd}_{2}(\mathrm{Allyl})_{2}\right]^{+}$gemacht. Offensichtlich tritt dieser Effekt nur bei sterisch sehr anspruchsvollen Substituenten am Imidazol auf; bislang (2,6-Dimethylphenyl-, 2,4,6-Trimethylphenyl- und sogar 2,6-Diisopropylphenyl-Substiuenten) wurde nur quasi-planares Verhalten des Rückgrats beobachtet.

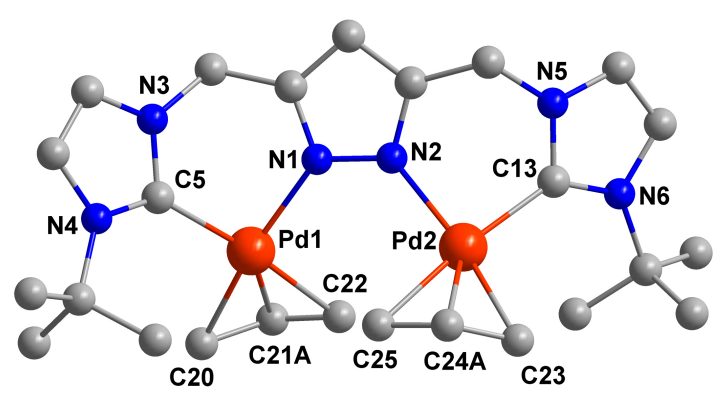

Abbildung 4.11: Festkörperstruktur von $\left[\mathrm{L}^{1} \mathrm{Pd}_{2}(\mathrm{Allyl})_{2}\right] \mathrm{BF}_{4}$. Aus Gründen der Übersichtlichkeit sind die Wasserstoffatome, das Gegenion und die Lösungsmittelmoleküle nicht dargestellt.

Nach der Umsetzung von $\left[\left(\mathrm{L}^{6}\right)_{2} \mathrm{Ag}_{4}\right]\left(\mathrm{PF}_{6}\right)_{2}$ mit Allylpalladium(II)-chlorid Dimer konnten durch Diethylether-Diffusion in eine Acetonitrillösung des Rohproduktes Einkristalle erhalten werden, die für eine röntgenographische Analyse geeignet waren. Hierbei bildete sich der Komplex $\left[\left(\mathrm{HL}^{6}\right)(\mathrm{MeCN}) \mathrm{Pd}\right]_{2}\left(\mathrm{PF}_{6}\right)_{4}$ (Abbildung 4.12). Ein neues Strukturmotiv für die Pyrazol-NHC-Hybridliganden wurde damit gefunden. Solch ein Komplex findet zwar keine katalytische Anwendung, allerdings wäre 
der Vergleich mit den isoelektronischen Ni-Komplexen, die als nächstes in diesem Abschnitt beschrieben werden, bezüglich ihrer Struktur von Interesse.

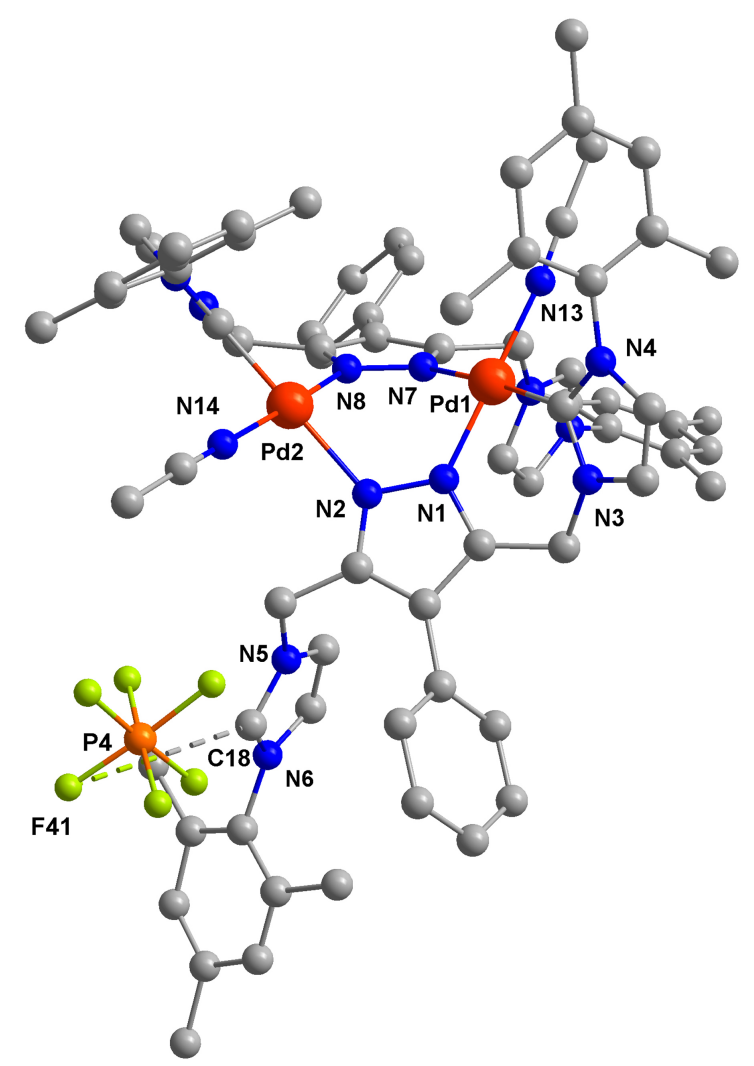

Abbildung 4.12: Festkörperstruktur von $\left[\left(\mathrm{HL}^{6}\right)(\mathrm{MeCN}) \mathrm{Pd}\right]_{2}\left(\mathrm{PF}_{6}\right)_{4}$. Aus Gründen der Übersichtlichkeit sind die Wasserstoffatome und einige Gegenionen nicht dargestellt.

Der neuartige Komplex $\left[\left(\mathrm{HL}^{6}\right)(\mathrm{MeCN}) \mathrm{Pd}\right]_{2}\left(\mathrm{PF}_{6}\right)_{4}$ setzt sich aus zwei Palladiumatomen und zwei Liganden zusammen. Jedes Palladiumatom ist durch zwei Pyrazolat-N-Atome, welche zu verschiedenen Liganden gehören und einem CarbenKohlenstoffatom koordiniert. Zusätzlich ist jedes Palladiumatom an einem Lösungsmittelmolekül (MeCN) gebunden. In Tabelle 4.8 sind charakteristische Bindungslängen und -winkel dieses Komplexes angegeben.

Etliche Versuche, diese Verbindung erneut zu kristallisieren, um so genügend analysenreines Material zu erhalten, blieben erfolglos. Das ESI-Massenspektrum aus dem Rohprodukt zeigt den höchsten Peak bei einem $m / z$-Verhältnis von 835a.u., welcher mit Hilfe des Isotopenmusters einem binuklearen Pd-Komplex, nämlich $\left[\mathrm{C}_{41} \mathrm{H}_{45} \mathrm{~N}_{6} \mathrm{Pd}_{2}\right]^{+}$zugeordnet werden kann. Dies ist genauso aus dem hochauflösenden Massenspektrum mit einem $m / z$-Verhältnis von 835.1786a.i. zu entnehmen. Das 
Tabelle 4.8: Ausgewählte Bindungslängen und -winkel von $\left[\left(\mathrm{HL}^{6}\right)(\mathrm{MeCN}) \mathrm{Pd}\right]_{2}\left(\mathrm{PF}_{6}\right)_{4}$. Standardabweichungen sind in Klammern angegeben.

\begin{tabular}{|c|c|c|}
\hline$\left[\left(\mathrm{HL}^{6}\right)(\mathrm{MeCN}) \mathrm{Pd}\right]_{2}\left(\mathrm{PF}_{6}\right)_{4}$ & Bindungslänge $[\AA]$ & Winkel $\left[^{\circ}\right]$ \\
\hline $\mathrm{Pd} 1-\mathrm{N} 1$ & $1.952(5)$ & \\
\hline Pd1 - C5 & $1.977(7)$ & \\
\hline Pd1 - N13 & $2.009(6)$ & \\
\hline $\mathrm{Pd} 1-\mathrm{N} 7$ & $2.062(5)$ & \\
\hline $\mathrm{Pd} 2-\mathrm{N} 8$ & $1.958(5)$ & \\
\hline $\mathrm{Pd} 2-\mathrm{C} 53$ & $1.971(7)$ & \\
\hline $\mathrm{Pd} 2-\mathrm{N} 14$ & $2.001(6)$ & \\
\hline $\mathrm{Pd} 2-\mathrm{N} 2$ & $2.089(5)$ & \\
\hline N5 - C18 & $1.313(8)$ & \\
\hline N6 - C18 & $1.346(8)$ & \\
\hline N12 - N2 & $1.395(6)$ & \\
\hline C5 - Pd1 - N1 & & $84.4(3)$ \\
\hline C5 - Pd1 - N13 & & $94.7(3)$ \\
\hline N1 - Pd1 - N13 & & $178.1(2)$ \\
\hline $\mathrm{C} 18-\mathrm{H} 18 \cdots \mathrm{F} 41$ & $3.098(9)$ & 155.0 \\
\hline
\end{tabular}

${ }^{1} \mathrm{H}-\mathrm{NMR}$ Spektrum bei RT zeigt stark verbreiterte Signale. Sowohl im ${ }^{1} \mathrm{H}-\mathrm{NMR}$ als auch im ${ }^{13} \mathrm{C}-\mathrm{NMR}$ Spektrum sind jedoch im erwarteten Allyl-Bereich einige Signale $\mathrm{zu}$ beobachten, die dem Komplex $\left[\left(\mathrm{HL}^{6}\right)(\mathrm{MeCN}) \mathrm{Pd}\right]_{2}\left(\mathrm{PF}_{6}\right)_{4}$ nicht zugeordnet werden können. Aufgrund der wenig aussagekräftigen spektroskopischen Daten lässt sich keine Aussage treffen, ob sich bei dieser Umsetzung ausschließlich der Komplex $\left[\left(\mathrm{HL}^{6}\right)(\mathrm{MeCN}) \mathrm{Pd}\right]_{2}\left(\mathrm{PF}_{6}\right)_{4}$ gebildet hat. Bei der gewonnenen Kristallstruktur scheint es sich um ein Nebenprodukt zu handeln.

Ausgehend vom chiralen $\left[\left(\mathrm{L}^{10}\right)_{2} \mathrm{Ag}_{4}\right]\left(\mathrm{BF}_{4}\right)_{2}$ bzw. $\left[\left(\mathrm{L}^{11}\right)_{2} \mathrm{Ag}_{4}\right]\left(\mathrm{PF}_{6}\right)_{2}$ gelang die Darstellung eines äquivalentes Allylpalladiumkomplexes $\left[\mathrm{L}^{10} \mathrm{Pd}_{2}(\mathrm{Allyl})_{2}\right] \mathrm{BF}_{4} \mathrm{bzw}$. $\left[\mathrm{L}^{11} \mathrm{Pd}_{2}(\mathrm{Allyl})_{2}\right] \mathrm{PF}_{6}$. NMR-spektroskopische Daten deuten auf die Bildung dieser Komplexe hin. Das Vorliegen verschiedener isomerer Formen in Lösung, welche bei den Allylpalladiumkomplexen mit achiralen NHC-Pyrazol-Liganden beobachtet wurde, ist hier genauso der Fall. Versuche, weitere Allylpalladiumkomplexe zu kristallisieren blieben erfolglos.

Es konnten insgesamt einige Palladiumkomplexe der Zusammensetzung $\left[\mathrm{L}^{\mathrm{x}} \mathrm{Pd}_{2}(\mathrm{Allyl})_{2}\right]^{2+}$ synthetisiert werden, wobei zwei Festkörperstrukturen mit Hilfe der Röntgenkristallstrukturanalyse bis jetzt erhalten werden konnten. Durch detaillierte NMR-spektroskopische Untersuchungen konnten die (Allyl)Palladiumkomplexe trotzdem eindeutig identifiziert werden. Zur Charakterisie- 
rung wurden zusätzlich hochauflösende Massenspektren aufgenommen, wodurch die Bildung der gewünschten Komplexe bestätigt wird.

Bei den bisherigen Komplexierungsversuchen wurde als Palladium-Vorläuferkomplex Allylpalladium(II)-chlorid verwendet, da dieses häufig zur Darstellung von katalytisch aktiven Palladium-Komplexen führen kann. Allylpalladiumchlorid liegt auch in Lösung als Dimer vor. Diese dimere Struktur kann im Fall einer Komplexierung aufgebrochen werden und die frei werdende Koordinationsstelle am Palladium kann von einen anderen Donor-Liganden eingenommen werden. Der Allyl-Ligand am Palladium kann gegen andere Allyl-Derivate ausgetauscht werden, und somit als Substrat für eine Palladium-katalysierte Allylübertragung fungieren.

Außerdem wurde die Darstellung von Palladiumkomplexen ausgehend von $\left[\mathrm{H}_{4} \mathrm{~L}^{\mathrm{x}}\right] \mathrm{Cl}_{3}$ und Palladium(II)-acetat in Anlehnung an Literatur [126] und unter Variation der Reaktionbedingungen untersucht. So wurden Versuche in Acetonitril, Dimethylsulfoxid, Chloroform oder in Lösungsmittel-Gemischen durchgeführt. Ein erhebliches Problem ergibt sich allerdings aus der Löslichkeit des jeweiligen Produkts, welche eine detaillierte Charakterisierung verhindert.

Das gleiche Problem der Löslichkeit ist bei den Umsetzungen von $\left[\left(\mathrm{L}^{\mathrm{x}}\right)_{2} \mathrm{Ag}_{4}\right]^{2+}$ mit dem Palladium(II)chlorid-Acetonitril-Addukt $\mathrm{PdCl}_{2}(\mathrm{MeCN})_{2}$ oder mit Palladium(II)chlorid $\mathrm{PdCl}_{2}$ aufgetreten. Reaktionen mit Palladium(II)chlorid-AcetonitrilAddukt wurden unter den für Transmetallierungsreaktionen eingeführten Reaktionsbedingungen durchgeführt. Reaktionen mit $\mathrm{PdCl}_{2}$ gingen vom Liganden aus, welcher unter Einwirkung einer Base (z.B. Kaliumcarbonat) deprotoniert wird.

Etliche Versuche eine Chlorid-Brücke bzw. Chloridliganden einzuführen sind somit gescheitert. Aufgrund der wenig aussagekräftigen Massenspektren, den schlechten NMR Spektren und vor allem wegen schlechter Löslichkeit wurde diese Verbindungsklasse bislang nicht weiter untersucht.

\subsubsection{Komplexe mit weiteren Metallen}

Nach erfolgreicher Koordination der Pyrazolat-NHC-Liganden an Palladium wurde versucht, Komplexe mit anderen Metallen mit d ${ }^{8}$-Konfiguration (wie Nickel(II)) darzustellen. Des Weiteren wurden Koordinationsversuche an Kupfer(II) durchgeführt. 


\section{Ni-Komplexe}

Bei der Umsetzung von $\left[\left(\mathrm{L}^{1}\right)_{2} \mathrm{Ag}_{4}\right]\left(\mathrm{BF}_{4}\right)_{2}$ mit $\mathrm{NiBr}_{2}$. DME in Acetonitril bei Raumtemperatur und unter Lichtausschluß erfolgt der Ligandentransfer auf Nickel und ein Ni-NHC-Komplex wird erhalten (Schema 4.8). Obwohl ein Ligand-zu-Nickel Verhältnis von 2:1 eingehalten wurde, zeigen die aufgenommenen ESI-Massenspektren zwar die Bildung eines binuklearen Ni-Komplexes allerdings mit einem Verhältnis zum Ligand von 2:2. Das ${ }^{1} \mathrm{H}-\mathrm{NMR}$ Spektrum zeigt stark verbreiterte Signale, obwohl die Komplexe mit Hilfe der Massenspektrometrie nachgewiesen werden konnten. Diese Verbreiterung der Signale ist auf paramagnetische Verunreinigungen zurückzuführen. Diverse Kristallisationsansätze lieferten keine messbaren Kristalle. Da keine röntgenstrukturanalytische Untersuchung durchgeführt werden konnte, ist eine genaue Aussage über die Struktur des hergestellten Nickel-NHC-Komplexes nicht möglich.

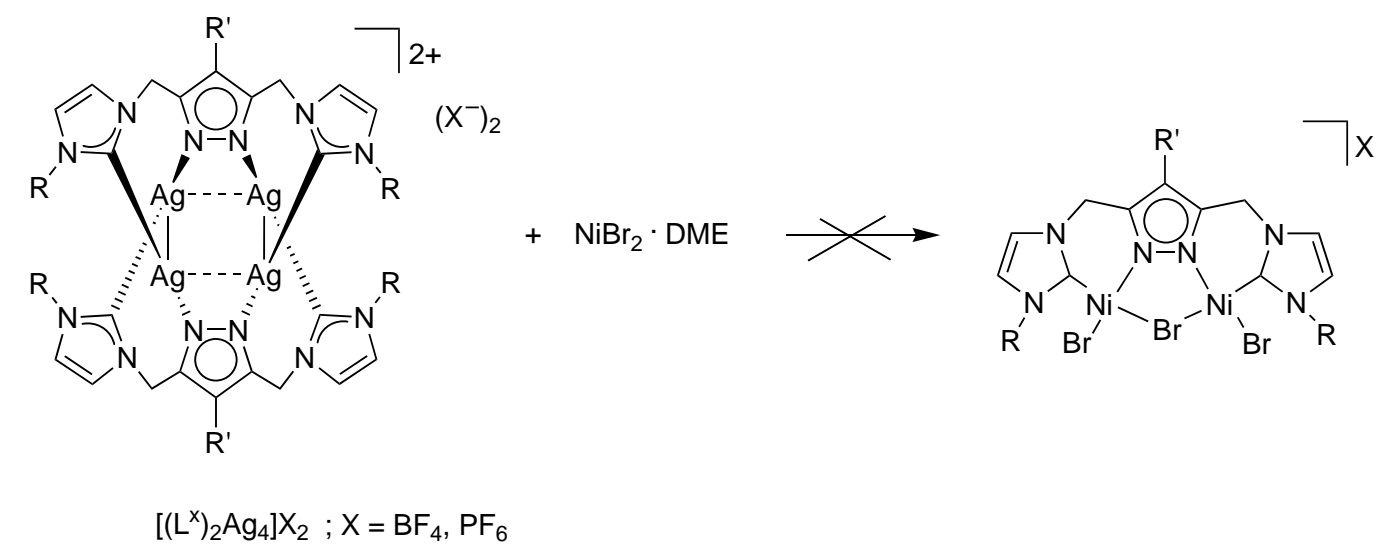

Schema 4.8: Denkbare Synthese von Ni-NHC-Komplexen unter Transmetallierung von Silber-NHC-Komplexen mit $\mathrm{NiBr}_{2} \cdot \mathrm{DME}$.

Das erwünschte Bindungsmotiv eines zweikernigen pyrazolatverbrückten Carbenkomplexes mit Verbrückungen aus dem Lösungsmittel $\left(\left[\mathrm{Ni}_{2} \mathrm{~L}(\mu-\mathrm{X})\right]\right.$; Abbildung 4.13), welches schon in der Literatur beschrieben wurde, konnte genauso wenig nachgewiesen werden. [37]

In der Literatur wurde von einem binuklearen Ni-Komplex mit ähnlichen NHCLiganden berichtet. [36] Ausgehend vom Liganden mit einer 2,6-DiisopropylphenylGruppe in den Seitenarmen und nach Deprotonierung wurde dieser mit $\mathrm{NiCl}_{2} \cdot \mathrm{DME}$ umgesetzt und lieferte einen Komplex der Zusammensetzung $\left[\mathrm{L}_{2} \mathrm{Ni}_{2}\right]$ (Abbildung 4.14). Die Struktur dieses Komplexes wurde ermittelt: die zwei Nickelatome sind jeweils durch zwei Carben- und zwei Pyrazolat-Stickstoffatome in einer verzerrten quadratisch-planaren Koordination gebunden, welche durch den sterischen 
Anspruch der 2,6-Diisopropylphenyl-Substituenten bedingt ist. Eine ähnliche Molekülstruktur wurde auch für die in dieser Arbeit hergestellten Ni-Komplexe erwartet. Obwohl diese Art von $\left[\mathrm{L}_{2} \mathrm{Ni}_{2}\right]$-Komplexen keine katalytische Anwendung findet, wäre ein Vergleich mit dem analogen Palladiumkomplex $\left[\mathrm{L}_{2} \mathrm{Pd}_{2}\right]$ weiterhin interessant.

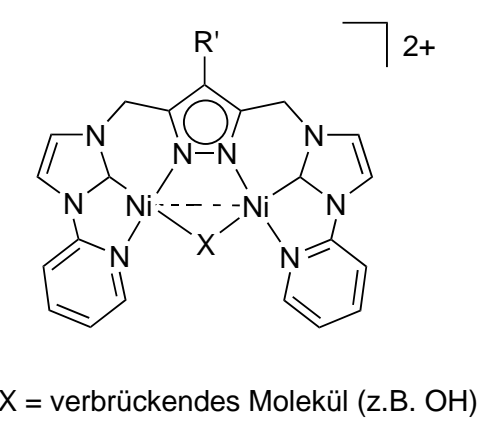

Abbildung 4.13: Bimetallischer Ni-NHC-Komplex für eine mögliche katalytische Anwendung.

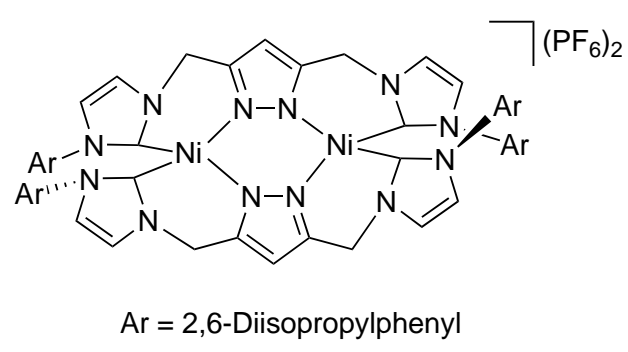

Abbildung 4.14: Ni-NHC-Komplex der Zusammensetzung $\left[\mathrm{L}_{2} \mathrm{Ni}_{2}\right]$.

Eine andere Möglichkeit zur Darstellung von Komplexen mit NHC-Liganden, die auch katalytisch von Interesse wären, ist die Umsetzung der Liganden mit Nickelocen $\left(\mathrm{NiCp}_{2}\right)$ (Schema 4.9). Diese Reaktion wird in Anlehnung an eine in der Literatur beschriebene Synthese durchgeführt. [127] Allerdings waren hier längere Reaktionszeiten erforderlich bis eine Farbänderung der Reaktionsmischung stattfand. Die Reaktion wurde für mehrere Verbindungen mit unterschiedlichen Substituenten am Imidazol getestet. Exemplarisch wird nachfolgend die Umsetzung am Beispiel der Ligandenvorstufe $\left[\mathrm{H}_{4} \mathrm{~L}^{5}\right] \mathrm{Cl}_{3}$ und in situ Umsalzen mit $\mathrm{NaBF}_{4}$ bzw. die direkte Umsetzung von $\left[\mathrm{H}_{3} \mathrm{~L}^{5}\right]\left(\mathrm{BF}_{4}\right)_{2}$ mit Nickelocen diskutiert. Die Bildung dieses NickelKomplexes ließ sich nicht gut mittels ${ }^{1} \mathrm{H}-\mathrm{NMR}$ Spektroskopie verfolgen. Das ${ }^{1} \mathrm{H}-$ NMR Spektrum zeigt stark verbreiterte Signale, vermutlich bedingt durch paramagnetische Verunreinigungen. Mit anderen analytischen Methoden lässt sich jedoch die Entstehung des Nickel-NHC-Komplexes des Typs [ $\left.\mathrm{LNi}_{2}\right]$ nachweisen: Das ESIMassenspektrum zeigt den höchsten Peak bei einem $m / z$-Verhältnis von 709a.u., 
welcher mit Hilfe des Isotopenmusters dem Fragment $\left[\mathrm{M}-\mathrm{BF}_{4}\right]^{+}$zugeordnet werden konnte. Es gelang bisher nicht Verbindungen des Typs $\left[\mathrm{LNi}_{2}\right] \mathrm{zu}$ isolieren oder zu kristallisieren, um Information über die Festkörperstruktur zu erhalten.

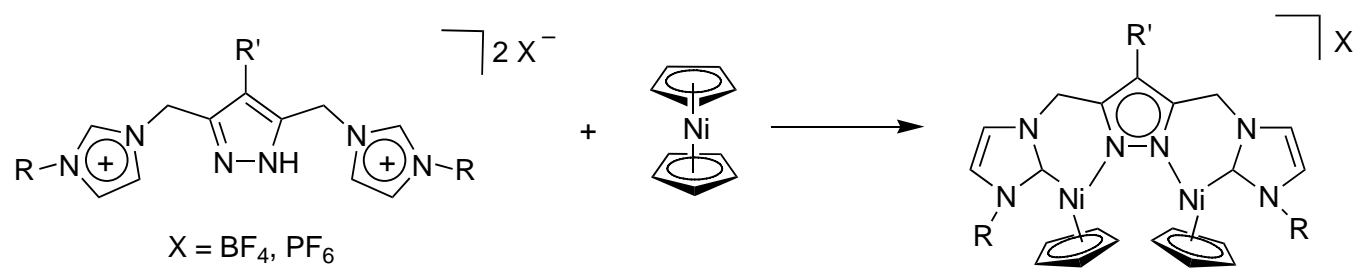

Schema 4.9: Denkbare Synthese von Ni-NHC-Komplexen aus der Umsetzung vom Liganden mit Nickelocen.

\section{Cu-Komplexe}

Zur Bildung von weiteren NHC-Komplexen kam als Metall Kupfer(II) zum Einsatz. Einige Cu-NHC-Komplexe finden Verwendung in allylischen Subtitutionsreaktionen. [128] Daher wäre es interessant die Effizienz in katalytischen Anwendungen im Vergleich zu den analogen Silber-NHC-Komplexen zu untersuchen.

Diverse synthetisierte Ag-NHC-Komplexe der Zusammensetzung $\left[\left(\mathrm{L}^{\mathrm{x}}\right)_{2} \mathrm{Ag}_{4}\right]^{2+}$ wurden mit $\mathrm{CuCl}_{2} \cdot 2 \mathrm{H}_{2} \mathrm{O}$ unter Lichtausschluss umgesetzt. Ein Nachweis der Produkte durch NMR gelang nicht. Es waren verbreiterte Signalen in NMR-Experimenten zu beobachten. Da Kupfer(II)-Ionen NMR-spektroskopisch schlecht erfassbar sind, ist die einzige Methode um eine Struktur exakt zu bestimmen die Röntgenstrukturanalyse, für die jedoch Einkristalle der zu untersuchenden Verbindung benötigt werden. Keiner der synthetisierten Komplexe konnte bis dato isoliert und strukturell charakterisiert werden. Massenspektroskopische Daten deuten nicht auf eine erfolgreiche Umsetzung hin. Sie zeigen häufig als einzigen Peak den des Liganden ohne gebundenes Metall. Daher konnte ein Gelingen der Reaktion auf diesem Wege nicht nachgewiesen werden.

\subsection{Katalyse}

Es wurde nun versucht, die katalytische Aktivität einiger der dargestellten Palladium-NHC-Komplexe und vor allem des neuen chiralen Palladium-NHCKomplexes in geeigneten Testreaktionen zu überprüfen. Dafür wurden ausgewählte Palladiumkomplexe $\left(\left[\mathrm{L}^{5} \mathrm{Pd}_{2}(\mathrm{Allyl})_{2}\right] \mathrm{PF}_{6}\right.$ und der chirale $\left.\left[\mathrm{L}^{11} \mathrm{Pd}_{2}(\mathrm{Allyl})_{2}\right] \mathrm{BF}_{4}\right)$ als 
Katalysatoren für eine allylische Substitution eingesetzt. Als geeignete Testreaktion wurde die Addition von Dimethylmalonat an 1,3-Diphenylallylacetat ausgewählt (Schema 4.10).<smiles>CC(=O)OC(/C=C/c1ccccc1)c1ccccc1</smiles><smiles>CCOC(=O)C(C(=O)OC)C(C)CC</smiles><smiles>COC(=O)C(C(=O)OC)[C@H](/C=C/c1ccccc1)c1ccccc1</smiles>

Schema 4.10: Palladium-katalysierte Addition von Dimethylmalonat an 1,3-Diphenylallylacetat.

Tabelle 4.9: Allylische Alkylierung von 1,3-Diphenylallylacetat mit Dimethylmalonat. ${ }^{a}$

\begin{tabular}{ccccc}
\hline Versuchsnr. & Katalysator & Additiv & Reaktionsbedingungen & Konversion $^{\mathrm{b}}$ \\
\hline 1 & {$\left[\mathrm{~L}^{5} \mathrm{Pd}_{2}(\text { Allyl })_{2}\right] \mathrm{PF}_{6}$} & - & THF; RT; $48 \mathrm{~h}$ & - \\
2 & {$\left[\mathrm{~L}^{11} \mathrm{Pd}_{2}(\text { Allyl })_{2}\right] \mathrm{PF}_{6}$} & - & THF; RT; $48 \mathrm{~h}$ & - \\
3 & {$\left[\mathrm{~L}^{5} \mathrm{Pd}_{2}(\text { Allyl })_{2}\right] \mathrm{PF}_{6}$} & $\mathrm{PPh}_{3}$ & THF; RT; $1 \mathrm{~h}$ & $100 \%$ \\
4 & {$\left[\mathrm{~L}^{5} \mathrm{Pd}_{2}(\text { Allyl })_{2}\right] \mathrm{PF}_{6}$} & - & THF; $45^{\circ} \mathrm{C} ; 48 \mathrm{~h}$ & - \\
5 & {$\left[\mathrm{~L}^{11} \mathrm{Pd}_{2}(\text { Allyl })_{2}\right] \mathrm{PF}_{6}$} & - & THF; $45^{\circ} \mathrm{C} ; 48 \mathrm{~h}$ & - \\
6 & {$\left[\mathrm{~L}^{11} \mathrm{Pd}_{2}(\text { Allyl })_{2}\right] \mathrm{PF}_{6}$} & $\mathrm{PPh}_{3}$ & THF; $45^{\circ} \mathrm{C} ; 1 \mathrm{~h}$ & $100 \%$ \\
\hline
\end{tabular}

Legende: ${ }^{a}$ Reaktion des Katalysators (2mol\%) mit 1,3-Diphenylallylacetat (1.0 mmol), Dimethylmalonat $(3.0 \mathrm{mmol})$ und $\mathrm{NaH}(3.0 \mathrm{mmol}){ }^{\mathrm{b}}$ Bestimmt durch ${ }^{1} \mathrm{H}-\mathrm{NMR}$ aus der Reaktionsmischung.

Der jeweilige Katalysator wurde bei Raumtemperatur mit 1,3-Diphenylallylacetat in THF suspendiert und nacheinander mit $\mathrm{NaH}$ und schließlich Dimethylmalonat versetzt. Die Experimente wurden stöchiometrisch und unter inerten Bedingungen durchgeführt. Einen Überblick über die Ergebnisse der jeweiligen Experimente gibt Tabelle 4.9 wieder.

Sowohl der Komplex $\left[\mathrm{L}^{5} \mathrm{Pd}_{2}(\mathrm{Allyl})_{2}\right] \mathrm{PF}_{6}$ als auch der neuartige chirale Komplex $\left[\mathrm{L}^{11} \mathrm{Pd}_{2}(\text { Allyl })_{2}\right] \mathrm{PF}_{6}$ zeigen bei dieser Reaktion keine Reaktivität. Nur nach Zugabe von $\mathrm{PPh}_{3}$ konnte eine vollständige Umwandlung nachgewiesen werden. Es wird vermutet, dass das Phosphan an das Palladium koordiniert. Somit entsteht ein Komplex, welcher die Umsetzung ermöglicht. 


\section{Kapitel 5}

\section{Zusammenfassung}

Gezieltes Ligandendesign gewinnt in der chemischen Forschung immer mehr an Bedeutung. Es existiert eine Vielzahl an Liganden, die sich am Rückgrat, in der Art und Anzahl ihrer Donoratome sowie den gebundenen Substituenten an den Donoratomen unterscheiden. Die Unterklasse der $N$-heterozyklischen Carbene (NHCs) eignet sich hervorragend als Ligand für die Komplexsynthese und wurde zu diesem Zweck eingeführt. Das Pyrazol stellt aufgrund seiner vielseitigen Funktionalisierbarkeit ein ideales Basismolekül für die Ligandsynthese dar. Unter Verwendung dieser Grundbausteine konnten im Rahmen dieser Arbeit mehrere PyrazolNHC-Hybridliganden mit unterschiedlichen arylischen und aliphatischen Substituenten synthetisiert werden. Die Einführung einer Phenylgruppe an der Pyrazol-C ${ }^{4}$ Position hat zu keiner signifikanten Verbesserung bezüglich der Löslichkeit oder Kristallisationseigenschaften der Verbindungen geführt. Es stellte sich heraus, dass die Tetrafluoroborat-Verbindungen besser kristallisieren als die mit Hexafluorophosphat. Der Folgeschritt der Synthese über eine Salzmetathese erwies sich als äußerst sinnvoll, da die Hydrochlorid-Ligandvorläufer stark hygroskopisch sind. Somit war eine einfachere Handhabung möglich. Zusätzlich bietet dieser Schritt für die nachfolgende Komplexsynthese besser geeignete, weniger stark koordinierende Gegenionen an. Anfänglich fokussierte sich die Arbeit auf sperrig anspruchsvolle Substituenten wie tert-Butyl und 1-Adamantyl. Die zusätzliche Einführung von Chiralität in dieser Art von Systemen führte zu neuartigen Ligandsystemen (Abbildung 5.1).

Ziel dieser Arbeit war es, Übergangsmetall-Komplexe mit diesen NHC-Liganden zu synthetisieren. Es gibt einige Möglichkeiten zur Herstellung von ÜbergangsmetallNHC-Komplexen. Als Methode der Wahl hat sich die Transmetallierungsreaktion von Silber-NHC-Komplexen herausgestellt. Diese verläuft unter milden Bedingun- 


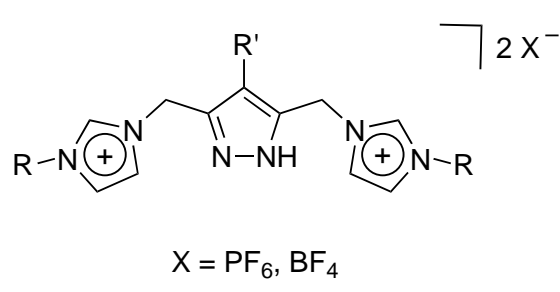

$\left[\mathrm{H}_{3} \mathrm{~L}^{\mathrm{1}}\right] \mathrm{X}_{2}\left(\mathrm{R}=t \mathrm{Bu}, \mathrm{R}^{\prime}=\mathrm{H}\right)$

$\left[\mathrm{H}_{3} \mathrm{~L}^{2}\right] \mathrm{X}_{2}\left(\mathrm{R}=t \mathrm{Bu}, \mathrm{R}^{\prime}=\mathrm{Ph}\right)$

$\left[\mathrm{H}_{3} \mathrm{~L}^{3}\right] \mathrm{X}_{2}\left(\mathrm{R}=1\right.$-Adamantyl, $\left.\mathrm{R}^{\prime}=\mathrm{H}\right)$

$\left[\mathrm{H}_{3} \mathrm{~L}^{4}\right] \mathrm{X}_{2}\left(\mathrm{R}=1\right.$-Adamantyl, $\left.\mathrm{R}^{\prime}=\mathrm{Ph}\right)$

$\left[\mathrm{H}_{3} \mathrm{~L}^{5}\right] \mathrm{X}_{2}\left(\mathrm{R}=\right.$ Mes, $\left.\mathrm{R}^{\prime}=\mathrm{H}\right)$

$\left[\mathrm{H}_{3} \mathrm{~L}^{6}\right] \mathrm{X}_{2}\left(\mathrm{R}=\mathrm{Mes}, \mathrm{R}^{\prime}=\mathrm{Ph}\right)$

$\left[\mathrm{H}_{3} \mathrm{~L}^{7}\right] \mathrm{X}_{2}\left(\mathrm{R}=\right.$ 4-Nitrophenyl, $\left.\mathrm{R}^{\prime}=\mathrm{Ph}\right)$

$\left[\mathrm{H}_{3} \mathrm{~L}^{8}\right] \mathrm{X}_{2}\left(\mathrm{R}=1\right.$-Benzyl, $\left.\mathrm{R}^{\prime}=\mathrm{H}\right)$

$\left[\mathrm{H}_{3} \mathrm{~L}^{9}\right] \mathrm{X}_{2}\left(\mathrm{R}=1\right.$-Benzyl, $\left.\mathrm{R}^{\prime}=\mathrm{Ph}\right)$

$\left[\mathrm{H}_{3} \mathrm{~L}^{10}\right] \mathrm{X}_{2}\left(\mathrm{R}=(\mathrm{S})\right.$-1-Phenylethyl, $\left.\mathrm{R}^{\prime}=\mathrm{H}\right)$

$\left[\mathrm{H}_{3} \mathrm{~L}^{11}\right] \mathrm{X}_{2}\left(\mathrm{R}=(\mathrm{S})-1-\right.$ Phenylethyl, $\left.\mathrm{R}^{\prime}=\mathrm{Ph}\right)$

Abbildung 5.1: Verwendete Ligandsysteme.

gen und ist mit nur geringen apparativen Aufwand verbunden. Ein Problem ergab sich allerdings aus den Ausbeuten der Synthesen, welche meist niedrig waren.

Silber-NHC-Komplexe sind nicht nur als Carbentransfer-Reagenzien verwendbar, sondern sind selbst aufgrund ihrer oft ungewöhnlichen strukturellen und optischen Eigenschaften von Interesse. Sie liegen oft als mehrkernige Aggregate vor, wobei die strukturelle Vielfalt enorm ist. Die Nuklearität hängt dabei unter anderem vom unterschiedlichen Substituenten an den Seitenarmen und dem verwendeten Lösungsmittel ab. Somit waren interessante Strukturmotive im Festkörper zu beobachten. Tetranukleare Silber-NHC-Komplexe sind häufig im Bezug auf NHC-Liganden beschrieben worden. Komplexe der Zusammensetzung $\left[\left(\mathrm{L}^{\mathrm{x}}\right)_{2} \mathrm{Ag}_{4}\right]^{2+}$ konnten aus der Reaktion zwischen dem jeweiligen Liganden $\left[\mathrm{H}_{3} \mathrm{~L}^{\mathrm{x}}\right](\mathrm{X})_{2}$ mit Silber(I)oxid in Acetonitril erhalten werden. Das übliche Bindungsmotiv beinhaltet jedes SilberAtom in einer Koordination von je einem Pyrazolat-N-Atom und einem CarbenKohlenstoffatom mit einer nahezu rechteckigen Anordnung der Silber-Atome. Viele dieser Komplexe wurden in dieser Arbeit strukturell charakterisiert. Wenn die Reaktion des Ligandenvorläufers mit Silber(I)oxid in Aceton durchgeführt wird, wird das Produkt $\left[\left(\mathrm{H}_{2} \mathrm{~L}^{1}\right)_{4} \mathrm{Ag}_{4}\right]^{8+}$ erhalten, welches ein Ligand-zu-Metall Verhältnis von 4:4 enthält. Die Silber-Atome sind nur an Pyrazolat-N-Atome gebunden, während alle Seitenarm-Imidazoliumgruppen protoniert und nicht koordinierend sind. Von diesem neuartigen Komplex sind Absorptions- und Emissionsspektren aufgenommen worden. Er zeigt deutliche Lumineszenz-Eigenschaften mit einer breiten Bande, zentriert bei etwa $310 \mathrm{~nm}$, wenn bei $\lambda_{e x}=265 \mathrm{~nm}$ angeregt wird. Für einige Verbindungen mit der Zusammensetzung $\left[\left(\mathrm{L}^{\mathrm{x}}\right)_{2} \mathrm{Ag}_{4}\right]^{2+}$ waren hingegen keine Absorptionsbanden zu sehen.

Sechskernige Silber-Komplexe mit NHC-Liganden wurden seltener beobachtet. In dieser Arbeit konnte ein neuartiger sechskerniger Silber(I)-Komplex isoliert werden. Eigentlich war es möglich im Fall des chiralen 1-Phenylethyl-Liganden zwei kristal- 
line Verbindungen mit unterschiedlichen Strukturen zu isolieren. In dieser Struktur sind vier von sechs Silberatomen je an ein Pyrazolat-N-Atom und ein CarbenKohlenstoffatom gebunden und zusätzlich von einem benachbarten Silber-Atom koordiniert. Die restlichen zwei Silber-Atome sind an zwei Carben-Kohlenstoffatome und zwei benachbarte Silber-Atome gebunden und die letzte Koordinationsstelle ist durch das Lösungsmittelmolekül besetzt. Die Wahl des Lösungsmittels ist hierbei entscheidend, ob im Festkörper $\left.\left[\left(\mathrm{L}^{10}\right)_{2} \mathrm{Ag}_{6} \text { (Aceton }\right)_{2}\right]^{4+}$ oder $\left[\left(\mathrm{L}^{10}\right)_{2} \mathrm{Ag}_{4}\right]^{2+}$ gebildet wird. Diese Ag-Komplexe weisen kein dynamisches Verhalten in Lösung auf. Der Versuch den hexanuklearen Komplex gezielt ausgehend vom Liganden und sukzessive Zugabe von $\mathrm{Ag}_{2} \mathrm{O}$ und teilweise von $\mathrm{AgBF}_{4}$ herzustellen ist fehlgeschlagen.

Zusammenfassend ist in Schema 5.1 die Zusammenhänge zwischen den einzelnen Silber-Strukturen zu sehen.

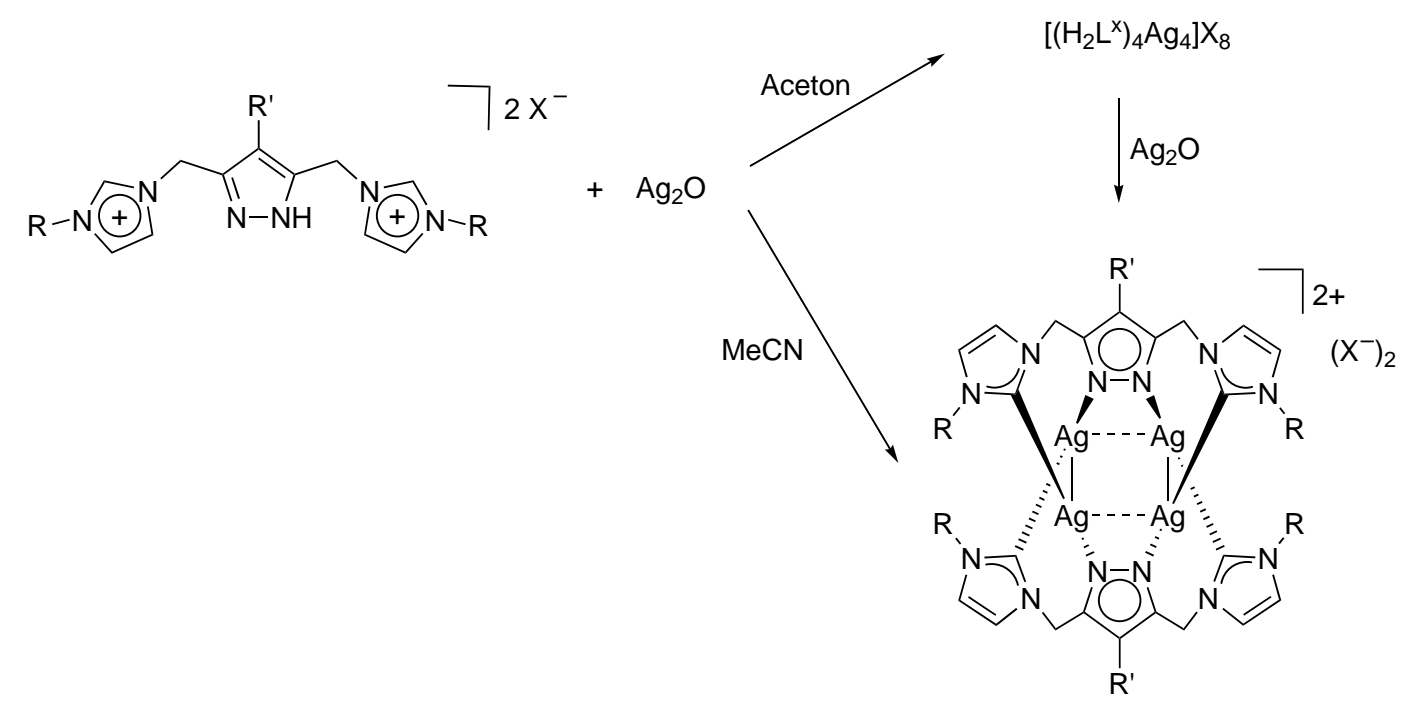

$\left[\left({ }^{\mathrm{X}}\right)_{2} \mathrm{Ag}_{4}\right] \mathrm{X}_{2}$

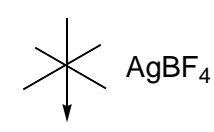

$\left[\left(L^{\mathrm{x}}\right)_{2} \mathrm{Ag}_{6}(\text { (Aceton) })_{2}\right] \mathrm{X}_{4}$

Schema 5.1: Diverse Strukturmotive der synthetisierten Silber(I)-NHC-Komplexe.

Über Transmetallierungsreaktionen mit Palladiumsalzen konnten die PyrazolNHC-Hybridliganden an Palladium koordiniert werden. In den Fällen von $\left[\mathrm{L}^{5} \mathrm{Pd}_{2}(\mathrm{Allyl})_{2}\right] \mathrm{PF}_{6}$ und $\left[\mathrm{L}^{1} \mathrm{Pd}_{2}(\mathrm{Allyl})_{2}\right] \mathrm{BF}_{4}$ konnte mittels Röntgenkristallstrukturanalyse die bimetallische Struktur der Komplexe nachgewiesen werden. Kom- 
plex $\left[\mathrm{L}^{5} \mathrm{Pd}_{2}(\mathrm{Allyl})_{2}\right] \mathrm{PF}_{6}$ wurde hinsichtlich seines Verhaltens in Lösung NMRspektroskopisch untersucht. Diese Untersuchungen bestätigen die Existenz zweier isomerer Formen des Palladiumkomplexes, was sich mit vorangegangenen Beobachtungen bei änlichen Allylkomplexen deckt. Sie haben eine Rotation für die Allylliganden ergeben mit $k_{\text {hin }}=0.20 s^{-1}$ und $k_{r \ddot{c} c k}=0.22 s^{-1}$. Der Synthese der NHCKomplexe folgte eine vollständige spektroskopische Charakterisierung. Außerdem wurde ein neues Strukturmotiv für die Pyrazol-NHC-Hybridliganden gefunden. Bei der Umsetzung von $\left[\left(\mathrm{L}^{6}\right)_{2} \mathrm{Ag}_{4}\right]\left(\mathrm{PF}_{6}\right)_{2}$ mit Allylpalladium(II)-chlorid Dimer wurde die Festkörperstruktur von $\left[\left(\mathrm{HL}^{6}\right)(\mathrm{MeCN}) \mathrm{Pd}\right]_{2}\left(\mathrm{PF}_{6}\right)_{4}$ erhalten. Solch ein Komplex mit der Zusammensetzung $\mathrm{L}_{2} \mathrm{Pd}_{2}$ findet keine katalytische Anwendung; allerdings wäre der Vergleich mit den isoelektronischen Ni-Komplexen $\left(\mathrm{L}_{2} \mathrm{Ni}_{2}\right)$ bezüglich ihrer Struktur von Interesse. Es gelang nicht weitere Allylpalladiumkomplexe zu kristallisieren.

Des Weiteren wurden die Komplexe $\left[\mathrm{L}^{5} \mathrm{Pd}_{2}(\mathrm{Allyl})_{2}\right] \mathrm{PF}_{6}$ und $\left[\mathrm{L}^{11} \mathrm{Pd}_{2}(\mathrm{Allyl})_{2}\right] \mathrm{PF}_{6}$ auf ihre katalytische Aktivität hin untersucht. Es existiert zwar eine Vielzahl bekannter Katalysatoren. Trotzdem gilt als Ziel der heutigen Forschung, neue katalytische Systeme zu entwickeln und die breite Palette der bereits verwendeten Katalysatoren zu erweitern. Als Testreaktion wurde die Palladium-katalysierte Allylübertragung ausgewählt, da diese mechanistisch gut verstanden ist. Erste Katalysetests wurden bei Raumtemperatur und auf $45^{\circ} \mathrm{C}$ durchgeführt. Diese haben jedoch keine katalytische Aktivität für die bimetallische Komplexe gezeigt. Vielversprechend dagegen war die Umsetzung in Gegenwart von $\mathrm{PPh}_{3}$. Es wird vermutet, dass sich hierbei eine Palladium- $\mathrm{PPh}_{3}$-Spezies bildet. Somit spielt $\mathrm{PPh}_{3}$ die entscheidene Rolle bei der Umsetzung und nicht der eingesetzte Komplex.

Nachdem eine breite Palette von Pyrazol-NHC-Liganden synthetisiert und ihre Übergangsmetallkomplexe dargestellt wurden, sollte weiterhin nach geeigneten katalytischen Anwendungen gesucht werden. 


\section{Teil II}

Experimenteller Teil 



\section{Kapitel 6}

\section{Allgemeine Arbeitstechnik}

Alle Reaktionen zur Liganden- und Silber-Komplexdarstellung sowie die Transmetallierungsreaktionen wurden, soweit nicht anders vermerkt, in kommerziell erhältlichen Lösungsmitteln (Reinheitsgrad p.a.), unter normalen Laborbedingungen durchgeführt.

Alle katalytischen Reaktionen wurden unter Ausschluss von Luft und Feuchtigkeit in einer Stickstoffatmosphäre mit Hilfe der Schlenktechnik durchgeführt. Unter solchen Bedingungen wurden Glasgeräte vor Gebrauch sekuriert. Sämtliche verwendeten Lösungsmittel wurden nach Standardvorschriften getrocknet, destilliert und unter einer Stickstoffatmosphäre aufbewahrt. Diethylether und THF wurden über Kaliumhydroxid vorgetrocknet und anschließend über Natrium/Kalium und Kalium destilliert. Dichlormethan wurde über Calciumhydrid destilliert.

\subsection{Analytik}

Die Aufnahmen der NMR Spektren erfolgten auf einem Bruker Avance $200\left({ }^{1} \mathrm{H}-\right.$ NMR: 200MHz), einem Bruker Avance $300\left({ }^{1} \mathrm{H}-\mathrm{NMR}: 300 \mathrm{MHz} ;{ }^{13} \mathrm{C}-\mathrm{NMR}: 75 \mathrm{MHz}\right)$, einem Bruker 400 und einem Bruker Avance DRX $500\left({ }^{1} \mathrm{H}-\mathrm{NMR}\right.$ : 500MHz; ${ }^{13} \mathrm{C}-\mathrm{NMR}$ : $126 \mathrm{MHz} ;{ }^{109} \mathrm{Ag}-\mathrm{NMR}$ : $\left.23 \mathrm{MHz}\right)$. Chemische Verschiebungen werden in ppm relativ zum Restprotonensignal des Lösungsmittels angegeben: $\mathrm{CDCl}_{3}\left({ }^{1} \mathrm{H}\right.$ : $\left.7.24 \mathrm{ppm} ;{ }^{13} \mathrm{C}: 77.0 \mathrm{ppm}\right), \mathrm{MeCN}-d_{3}\left({ }^{1} \mathrm{H}: 1.94 \mathrm{ppm} ;{ }^{13} \mathrm{C}: 118.3 \mathrm{ppm}\right)$ und DMSO- $d_{6}$ $\left({ }^{1} \mathrm{H}: 2.50 \mathrm{ppm} ;{ }^{13} \mathrm{C}: 39.52 \mathrm{ppm}\right)$. [129, 130$]{ }^{15} \mathrm{~N}$ - und ${ }^{107 / 109} \mathrm{Ag}$-Resonanzen wurden indirekt durch HMQC-Experimente detektiert, [131] unter Verwendung jeweils von 33 und $166 \mathrm{~ms}$ als Transferdelays. Diese Kerne wurden außerdem bezüglich 
der allgemeinen $\Xi$-Skala referenziert; [132] indirekte Referenzierung: $\Xi\left(\mathrm{Me}^{15} \mathrm{NO}_{2}\right)$ $=10.136767$ und $\Xi$ (gesätt. $\left.{ }^{109} \mathrm{AgNO}_{3}\right)=4.653533$. In manchen Fällen wurden für die Zuordnung von Signalen 2D-Spektren aufgenommen, beispielsweise HH-NOESY oder (long range-) CH-COSY. Zusätzlich wurden HMQC-Experimente mit einem Transferdelay von $33 \mathrm{~ms}$ durchgeführt, um die Carbensignale sowie weitere quartäre ${ }^{13}$ C-Signale zuzuordnen. Die Messungen wurden bei RT durchgeführt, sofern nicht anders angegeben. Verwendete Abkürzungen für Signalmultiplizitäten: s = Singulett, $\mathrm{d}=$ Dublett, $\mathrm{t}=$ Triplett, $\mathrm{q}=$ Quartett, sept $=$ Septett, $\mathrm{m}=$ Multiplett, $\mathrm{br}$ $=$ breites Signal.

EI-Massenspektren wurden mit einem Massenspektrometer der Firma Finnigan, Modell MAT 8200 (70eV) und ESI-Massenspektren auf einem Thermo Finnigan Trace LCQ Spektrometer aufgenommen. Simulationen von Isotopenverteilungen wurden mit Hilfe des Programms Molecular Weight Calculator durchgeführt. [133] Angegeben ist jeweils das Verhältnis von Masse zu Ladung ( $m / z)$, bei dem die intensitätsstärkste Linie eines Isotopenmusters liegt.

Elementaranalysen wurden durch das Analytische Labor des Instituts für Anorganische Chemie der Universität Göttingen unter Verwendung des Gerätes Elementar Vario EL III durchgeführt. Die gerätebedingte Messgenauigkeit liegt bei $\pm 0.3 \%$.

UV/Vis- und Fluoreszenzspektren von den Silber-NHC-Komplexen in Lösung wurden jeweils an einem Jasco V-550 UV/Vis Spektrometer und einem Jasco FP-6500 Fluoreszenz Spektrometer in Küvetten aus Quarzglas mit einer Schichtdicke von $1 \mathrm{~cm}$ aufgenommen. Die Spektrenaufnahme erfolgte im Spektralbereich von 230 bis 600nm. Sämtliche Fluoreszenzspektren wurden am Institut für Organische und Biomolekulare Chemie im Arbeitskreis von Prof. Steinem aufgenommen.

Infrarotspektren wurden an einem Excalibur Series FTS 3000-Spektrometer (DIGILAB) aufgenommen. Die Messung der Proben erfolgte im Festkörper als Kaliumbromid-Pressling. Folgende Abkürzungen wurden für die Intensitätsangaben verwendet: vs $=$ sehr stark, $\mathrm{s}=$ stark, $\mathrm{m}=$ mittel, $\mathrm{w}=$ schwach, $\mathrm{br}=$ breit.

Schmelz- bzw. Zersetzungspunkte wurden in offenen Glaskapillaren an einem SRS OptiMelt Messgerät bestimmt und sind nicht korrigiert. Die Heizrate betrug $10 \mathrm{~K} \cdot \mathrm{min}^{-1}$.

\subsection{Verwendete Chemikalien}

Die im Folgenden aufgeführten Substanzen wurden nach literaturbekannten Verfahren hergestellt. Chemikalien, deren Darstellung nicht aufgeführt ist, wurden kommerziell erworben oder waren im Arbeitskreis vorhanden. 
$\mathrm{NaH}$ wurde als Dispersion in Mineralöl kommerziell erworben, mehrfach mit Hexan gewaschen und im Vakuum getrocknet.

$N$-substituierte Imidazole: 1-(2,4,6-Trimethylphenyl)- $1 H$-imidazol,

1-(tert-Butyl)-1H-imidazol, 1-(1-Adamantyl)- $1 H$-imidazol, 1-Benzyl-1 $H$-imidazol, 1-(1-Phenylethyl)-1 $H$-imidazol, 1-(4-Nitrophenyl)-1 $H$-imidazol. [44-46, 134$]$

3,5-Bis(chlormethyl)-1-(tetrahydropyran-2-yl)-1H-pyrazol, [39]

3,5-Bis(chlormethyl)-1-(tetrahydropyran-2-yl)-4-phenyl-1 $H$-pyrazol, [43] trans-Bis(acetonitril)palladium(II)chlorid, [135, 136]

Allylpalladium(II)-chlorid Dimer, [137, 138]

$\beta$-Methallylpalladium(II)-chlorid Dimer, [139]

$\operatorname{Bis}\left(\eta^{5}\right.$-cyclopentadienyl)nickel. [140]

\subsection{Allgemeine Arbeitsvorschriften}

\subsubsection{Synthese der Liganden als Hydrochloride}

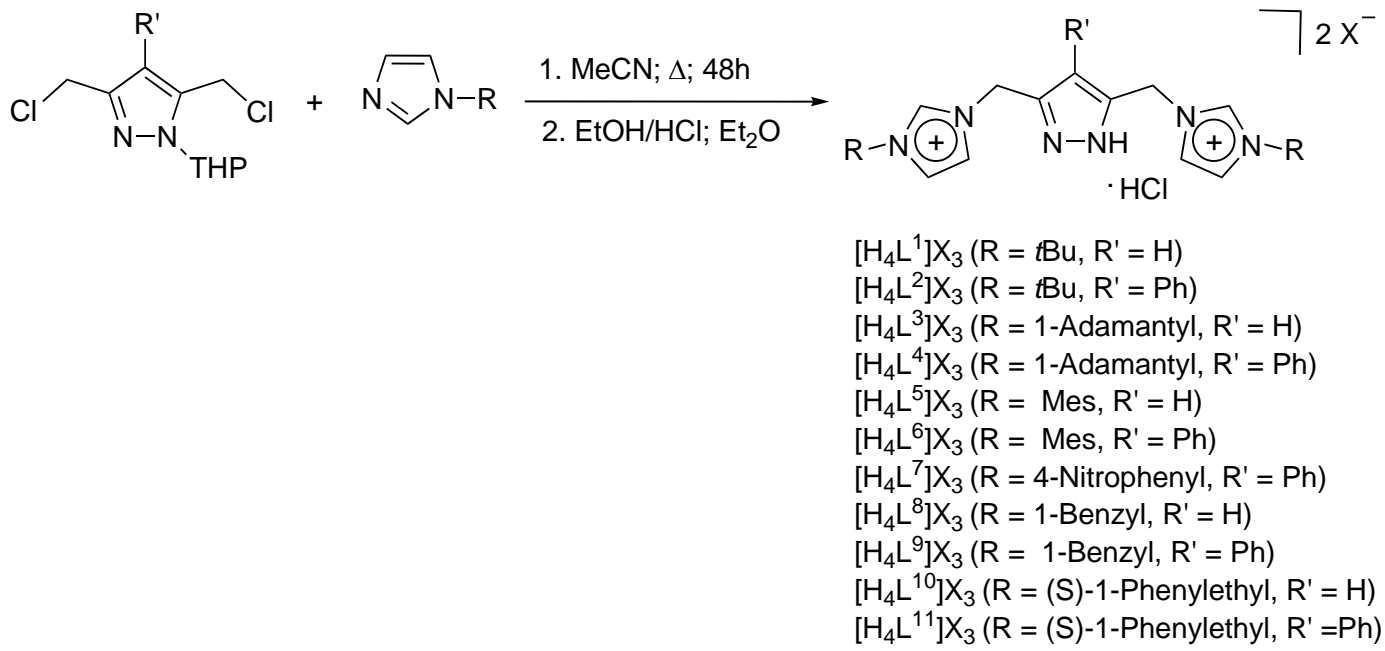

Basierend auf [35]

Eine Mischung aus 3,5-Bis(chlormethyl)-1-(tetrahydropyran-2-yl)-1 $H$-pyrazol (6) bzw. 3,5-Bis(chlormethyl)-1-(tetrahydropyran-2-yl)-4-phenyl-1 $H$-pyrazol (12) (25.0 mmol, 1.0 Äq) und dem entsprechenden $N$-substituierten Imidazol (75.0 mmol, 
3.0 Äq) werden in $100 \mathrm{ml}$ Acetonitril gelöst und für $48 \mathrm{~h}$ unter Rückfluss erhitzt. Nach dieser Zeit wird das Lösungsmittel unter vermindertem Druck entfernt und der Rückstand in einer Mischung aus Wasser (100ml) und Dichlormethan (100ml) aufgenommen. Die Phasen werden getrennt; die wässrige Phase wird dreimal mit Dichlormethan (je 50ml) ausgeschüttelt. Aus den vereinigten organischen Phasen kann der Überschuss des eingesetzten Imidazols zurückgewonnen werden. Die wässrige Phase wird unter Vakuum abgezogen und der Rückstand nochmal im Vakuum getrocknet. Dieser wird wiederum in möglichst wenig Ethanol (10 ml) aufgenommen und mit ethanolischer Salzsäure versetzt. Die Lösung wird über Nacht bei RT gerührt. Zur vollständigen Fällung des Produkts wird Diethylether (1.01) zugegeben. Das Feststoff wird durch Filtration abgetrennt, mit Diethylether gewaschen $(200 \mathrm{ml})$ und im Vakuum bei etwa $90^{\circ} \mathrm{C}$ getrocknet.

Diese erhaltenen weißen Feststoffe (Hydrochloride) sind stark hygroskopisch. Aufgrund der ausgeprägten Eigenschaft der Hydrochloride Feuchtigkeit aus der Umgebung zu binden, konnten keine zufriedenstellenden Elementaranalysen erhalten werden. Die Hydrochloride werden unmittelbar direkt zu dem Hexafluorophosphatbzw. Tetrafluoroborat-Salzen umgesetzt.

\section{3,5-Bis[1-(tert-butyl)imidazolium-1-ylmethyl]-pyrazol Hydrochlorid; $\left[\mathrm{H}_{4} \mathrm{~L}^{1}\right] \mathrm{Cl}_{3}$}

Ausbeute: $5.26 \mathrm{~g}(21.12 \mathrm{mmol}) \mathrm{Pz}^{\mathrm{H}}-\mathrm{THP} ; 7.47 \mathrm{~g}(16.66 \mathrm{mmol})\left[\mathrm{H}_{4} \mathrm{~L}^{1}\right] \mathrm{Cl}_{3} ; 78 \%$; hell gelber Feststoff.

${ }^{1} \mathbf{H}-\mathbf{N M R}\left(500 \mathrm{MHz}, \mathrm{DMSO}-d_{6}\right): \delta=1.58(\mathrm{~s}, 18 \mathrm{H}, t \mathrm{Bu}), 5.48\left(\mathrm{~s}, 4 \mathrm{H}, \mathrm{CH}_{2}\right), 6.54(\mathrm{~s}$, $\left.1 \mathrm{H}, \mathrm{CH}^{P z}\right), 7.87\left(\mathrm{t}, J=1.6 \mathrm{~Hz}, 2 \mathrm{H}, \mathrm{CH}^{I m}\right), 8.02\left(\mathrm{t}, J=1.6 \mathrm{~Hz}, 2 \mathrm{H}, \mathrm{CH}^{I m}\right), 9.74(\mathrm{~s}$, $\left.1 \mathrm{H}, \mathrm{CH}^{\operatorname{Im} 2}\right) \mathrm{ppm}$.

${ }^{13}$ C-NMR $\left(125 \mathrm{MHz}, \mathrm{DMSO}-d_{6}\right): \delta=28.9(t \mathrm{Bu}), 44.2\left(\mathrm{CH}_{2}\right), 58.9\left(\mathrm{C}^{t B u}\right), 104.9$ $\left(\mathrm{CH}^{P z 4}\right), 120.3\left(\mathrm{CH}^{I m}\right), 122.5\left(\mathrm{CH}^{I m}\right), 135.0\left(\mathrm{CH}^{I m 2}\right), 141.8\left(\mathrm{br}, \mathrm{C}^{P z 3,5}\right) \mathrm{ppm}$.

MS(FAB+) $: m / z(\%)=377(10)[\mathrm{M}-2 \mathrm{Cl}]^{2+}, 341(45)[\mathrm{M}-3 \mathrm{Cl}]^{3+}, 285(15)[\mathrm{M}-3 \mathrm{Cl}-$ $t \mathrm{Bu}]^{+}, 217$ (100) [M-3Cl-SA $]^{+}$.

Synthese von 3,5-Bis[1-( $t$ Bu)imidazolium-1-ylmethyl]-4-phenyl-pyrazol Hydrochlorid; $\left[\mathrm{H}_{4} \mathrm{~L}^{2}\right] \mathrm{Cl}_{3}$

Ausbeute: $2.00 \mathrm{~g}$ (6.15 mmol) $\mathrm{Pz}^{\mathrm{Ph}}$-THP; $2.70 \mathrm{~g}$ (5.13mmol) $\left[\mathrm{H}_{4} \mathrm{~L}^{2}\right] \mathrm{Cl}_{3} ; 83 \%$; hell gelber Feststoff. 
${ }^{1} \mathbf{H}-\mathbf{N M R}\left(500 \mathrm{MHz}, \mathrm{DMSO}-d_{6}\right): \delta=1.47(\mathrm{~s}, 18 \mathrm{H}, t \mathrm{Bu}), 5.53\left(\mathrm{~s}, 4 \mathrm{H}, \mathrm{CH}_{2}\right), 7.23$ $\left(\mathrm{m}, 2 \mathrm{H}, \mathrm{CH}^{o-P h}\right), 7.37\left(\mathrm{~m}, 3 \mathrm{H}, \mathrm{CH}^{m, p-P h}\right), 7.56\left(\mathrm{t}, J=1.7 \mathrm{~Hz}, 2 \mathrm{H}, \mathrm{CH}^{I m}\right), 7.93(\mathrm{t}$, $\left.J=1.7 \mathrm{~Hz}, 2 \mathrm{H}, \mathrm{CH}^{I m}\right), 9.39\left(\mathrm{t}, \mathrm{J}=1.6 \mathrm{~Hz}, 2 \mathrm{H}, \mathrm{CH}^{\operatorname{Im} 2}\right) \mathrm{ppm}$.

${ }^{13}$ C-NMR $\left(125 \mathrm{MHz}, \mathrm{DMSO}-d_{6}\right): \delta=28.9(t \mathrm{Bu}), 43.9\left(\mathrm{CH}_{2}\right), 59.5\left(\mathrm{C}^{t B u}\right), 118.9$ $\left(\mathrm{C}^{P z 4}\right), 120.3\left(\mathrm{CH}^{I m}\right), 122.6\left(\mathrm{CH}^{I m}\right), 127.5\left(\mathrm{CH}^{p-P h}\right), 128.7\left(\mathrm{CH}^{m-P h}\right), 129.2$ $\left(\mathrm{CH}^{o-P h}\right), 130.3\left(\mathrm{C}^{i-P h}\right), 135.0\left(\mathrm{CH}^{\operatorname{Im} 2}\right) \mathrm{ppm}$.

MS(ESI+): $m / z(\%)=417(7)[\mathrm{M}-3 \mathrm{Cl}-\mathrm{H}]^{+}, 361(75)[\mathrm{M}-3 \mathrm{Cl}-2 \mathrm{H}-t \mathrm{Bu}]^{+}, 305(66)$ $[\mathrm{M}-3 \mathrm{Cl}-\mathrm{H}-2 t \mathrm{Bu}]^{+}, 237(100)[\mathrm{M}-3 \mathrm{Cl}-2 \mathrm{H}-\mathrm{SA}]^{+}, 57(23)[t \mathrm{Bu}]^{+}$.

3,5-Bis[1-(1-adamantyl)imidazolium-1-ylmethyl]-pyrazol Hydrochlorid; $\left[\mathrm{H}_{4} \mathrm{~L}^{3}\right] \mathrm{Cl}_{3}$

Ausbeute: $3.09 \mathrm{~g}(12.40 \mathrm{mmol}) \mathrm{Pz}^{\mathrm{H}}$-THP; $7.25 \mathrm{~g}(11.96 \mathrm{mmol})\left[\mathrm{H}_{4} \mathrm{~L}^{3}\right] \mathrm{Cl}_{3} ; 96 \%$; weißer Feststoff.

${ }^{1} \mathbf{H}-\mathbf{N M R}\left(500 \mathrm{MHz}, \mathrm{DMSO}-d_{6}\right): \delta=1.71\left(\mathrm{~m}, 12 \mathrm{H}, \mathrm{CH}_{2}{ }^{A d}\right), 2.12\left(\mathrm{~m}, 12 \mathrm{H}, \mathrm{CH}_{2}{ }^{A d}\right)$, $2.20\left(\mathrm{~m}, 6 \mathrm{H}, \mathrm{CH}^{A d}\right), 5.48\left(\mathrm{~s}, 4 \mathrm{H}, \mathrm{CH}_{2}\right), 6.53\left(\mathrm{~s}, 1 \mathrm{H}, \mathrm{CH}^{P z}\right), 7.88(\mathrm{t}, J=1.8 \mathrm{~Hz}, 2 \mathrm{H}$, $\left.\mathrm{CH}^{I m}\right), 8.05\left(\mathrm{t}, J=1.8 \mathrm{~Hz}, 2 \mathrm{H}, \mathrm{CH}^{I m}\right), 9.70$ (br s, $\left.2 \mathrm{H}, \mathrm{CH}^{I m}\right) \mathrm{ppm}$.

${ }^{13} \mathbf{C}-\mathrm{NMR}\left(125 \mathrm{MHz}, \mathrm{DMSO}-d_{6}\right): \delta=28.8\left(\mathrm{CH}^{A d}\right), 34.7\left(\mathrm{CH}_{-} \mathrm{CH}_{2}-\mathrm{CH}^{A d}\right), 41.4$ $\left(\mathrm{C}-\mathrm{CH}_{2}^{A d}\right), 44.1\left(\mathrm{CH}_{2}\right), 59.2\left(\mathrm{C}^{A d}\right), 105.0\left(\mathrm{CH}^{P z}\right), 119.4\left(\mathrm{CH}^{I m}\right), 122.4\left(\mathrm{CH}^{I m}\right)$, $134.4\left(\mathrm{CH}^{\operatorname{Im} 2}\right), 141.5\left(\mathrm{C}^{P z 3,5}\right) \mathrm{ppm}$.

$\mathbf{M S}(\mathbf{F A B}+): m / z(\%)=497(40)[\mathrm{M}-3 \mathrm{Cl}-2 \mathrm{H}]^{+}, 363(8)[\mathrm{M}-3 \mathrm{Cl}-2 \mathrm{H}-\mathrm{Ad}]^{+}, 295(100)$ $[\mathrm{M}-3 \mathrm{Cl}-\mathrm{SA}]^{+}$.

IR (KBr): 3432 (s), 3121 (s), 3076 (s), 2910 (vs), 2850 (vs), 1634 (m), 1550 (s), 1451 (s), 1361 (m), 1345 (w), 1308 (s), $1286(\mathrm{w}), 1257$ (m), 1196 (m), 1189 (m), $1157(\mathrm{vs}), 1104(\mathrm{~s}), 1065(\mathrm{w}), 1023(\mathrm{w}), 997(\mathrm{~m}), 985(\mathrm{w}), 843(\mathrm{w}), 828(\mathrm{w}), 816$ $(\mathrm{w}), 795(\mathrm{~m}), 772(\mathrm{w}), 746(\mathrm{~m}), 700(\mathrm{w}), 688(\mathrm{~m}), 638(\mathrm{~m}), 622(\mathrm{w})$.

\section{3,5-Bis[1-(1-adamantyl)imidazolium-1-ylmethyl]-4-phenyl-pyrazol} Hydrochlorid; $\left[\mathrm{H}_{4} \mathrm{~L}^{4}\right] \mathrm{Cl}_{3}$

Ausbeute: $2.76 \mathrm{~g}(8.49 \mathrm{mmol}) \mathrm{Pz}^{\mathrm{Ph}}$-THP; $2.55 \mathrm{~g}(3.74 \mathrm{mmol})\left[\mathrm{H}_{4} \mathrm{~L}^{4}\right] \mathrm{Cl}_{3} ; 44 \%$; weißer Feststoff.

${ }^{1} \mathbf{H}-\mathrm{NMR}\left(500 \mathrm{MHz}, \mathrm{DMSO}-d_{6}\right): \delta=1.68\left(\mathrm{br} \mathrm{s}, 12 \mathrm{H}, \mathrm{CH}_{2}{ }^{A d}\right), 1.99(\mathrm{~m}, 12 \mathrm{H}$, $\mathrm{CH}_{2}{ }^{A d}$ ), 2.17 (br s, 6H, CH${ }^{A d}$ ), 5.56 (br s, $4 \mathrm{H}, \mathrm{CH}_{2}$ ), $7.24\left(\mathrm{~m}, 2 \mathrm{H}, \mathrm{CH}^{o-P h}\right.$ ), 7.37 
$\left(\mathrm{m}, 3 \mathrm{H}, \mathrm{CH}^{m, p-P h}\right), 7.58\left(\mathrm{t}, J=1.8 \mathrm{~Hz}, 2 \mathrm{H}, \mathrm{CH}^{I m}\right), 7.98\left(\mathrm{t}, J=1.7 \mathrm{~Hz}, 2 \mathrm{H}, \mathrm{CH}^{I m}\right)$, $9.48\left(\mathrm{~m}, 2 \mathrm{H}, \mathrm{CH}^{\operatorname{Im} 2}\right) \mathrm{ppm}$.

${ }^{13} \mathbf{C}-\mathrm{NMR}\left(125 \mathrm{MHz}, \mathrm{DMSO}-d_{6}\right): \delta=28.7\left(\mathrm{CH}^{A d}\right), 34.6\left(\mathrm{CH}-\mathrm{CH}_{2}-\mathrm{CH}^{A d}\right), 41.3$ $\left(\mathrm{C}-\mathrm{CH}_{2}^{A d}\right), 43.8\left(\mathrm{CH}_{2}\right), 59.1\left(\mathrm{C}^{A d}\right), 119.3\left(\mathrm{CH}^{I m}\right), 122.4\left(\mathrm{CH}^{I m}\right), 127.3\left(\mathrm{CH}^{p-P h}\right)$, $128.6\left(\mathrm{CH}^{m-P h}\right), 129.1\left(\mathrm{CH}^{o-P h}\right), 130.1\left(\mathrm{C}^{i-P h}\right), 118.8\left(\mathrm{C}^{P z 4}\right), 134.5,134.6(2 \mathrm{x}$ $\left.\mathrm{CH}^{\operatorname{Im} 2}\right), 138.7$ (br, $\left.\mathrm{C}^{P z 3,5}\right) \mathrm{ppm}$.

MS(ESI+) $: m / z(\%)=573(8)[\mathrm{M}-3 \mathrm{Cl}-2 \mathrm{H}]^{+}, 439(100)[\mathrm{M}-3 \mathrm{Cl}-\mathrm{Ad}-2 \mathrm{H}]^{+}, 135(70)$ $[\mathrm{Ad}]^{+}$.

\section{3,5-Bis[1-(2,4,6-trimethylphenyl)imidazolium-1-ylmethyl]-4-phenyl- pyrazol Hydrochlorid; \\ $\left[\mathrm{H}_{4} \mathrm{~L}^{6}\right] \mathrm{Cl}_{3}$}

Ausbeute: $2.00 \mathrm{~g}$ (6.15mmol) $\mathrm{Pz}^{\mathrm{Ph}}$-THP; $1.71 \mathrm{~g}(2.63 \mathrm{mmol})\left[\mathrm{H}_{4} \mathrm{~L}^{6}\right] \mathrm{Cl}_{3} ; 43 \%$. Durch Diethylether-Diffusion in eine Aceton/MeOH-Mischung (1:1) des Rohproduktes bei $+8^{\circ} \mathrm{C}$ konnten farblose Kristalle gewonnen werden, die zur Röntgenkristallstrukturanalyse geeignet waren.

${ }^{1} \mathbf{H}-\mathbf{N M R}\left(500 \mathrm{MHz}, \mathrm{DMSO}-d_{6}\right): \delta=1.88\left(\mathrm{~s}, 12 \mathrm{H}, \mathrm{CH}_{3}{ }^{A r 2,6}\right), 2.35\left(\mathrm{~s}, 3 \mathrm{H}, \mathrm{CH}_{3}{ }^{A r 4}\right)$, 5.65, $5.74\left(2 \times \mathrm{s}, 2 \mathrm{H}, \mathrm{CH}_{2}\right), 7.09\left(\mathrm{~s}, 2 \mathrm{H}, \mathrm{CH}^{A r 3,5}\right), 7.34\left(\mathrm{~m}, 3 \mathrm{H}, \mathrm{CH}^{m, p-P h}\right), 7.40$ $\left(\mathrm{m}, 2 \mathrm{H}, \mathrm{CH}^{o-P h}\right), 7.85\left(\mathrm{t}, J=1.8 \mathrm{~Hz}, 2 \mathrm{H}, \mathrm{CH}^{I m}\right), 7.88\left(\mathrm{br} \mathrm{s}, 2 \mathrm{H}, \mathrm{CH}^{I m}\right), 9.43,9.53$ $\left(2 \times \mathrm{s}, 1 \mathrm{H}, \mathrm{CH}^{\operatorname{Im} 2}\right), 14.38$ (br s, $\left.1 \mathrm{H}, \mathrm{NH}\right) \mathrm{ppm}$.

${ }^{13}$ C-NMR $\left(125 \mathrm{MHz}, \mathrm{DMSO}-d_{6}\right): \delta=16.7\left(\mathrm{CH}_{3}{ }^{A r 2,6}\right), 20.4\left(\mathrm{CH}_{3}{ }^{A r 4}\right), 42.7,46.0$ $\left(2 \times \mathrm{CH}_{2}\right), 119.4\left(\mathrm{C}^{P z 4}\right), 122.7,123.4,123.7,124.1\left(\mathrm{CH}^{I m}\right), 127.5\left(\mathrm{CH}^{p-P h}\right), 128.7$ $\left(\mathrm{CH}^{m-P h}\right), 128.9\left(\mathrm{CH}^{o-P h}\right), 129.0\left(\mathrm{CH}_{3}{ }^{A r 3,5}\right), 129.0\left(\mathrm{CH}^{A r 3,5}\right), 130.1\left(\mathrm{CH}^{A r 1}\right)$, $130.9\left(\mathrm{C}^{i-P h}\right), 133.7\left(\mathrm{br}, \mathrm{C}^{P z 3,5}\right), 134.1\left(\mathrm{C}^{A r 2,6}\right), 137.6\left(\mathrm{br}, \mathrm{CH}^{\operatorname{Im} 2}\right), 140.1\left(\mathrm{CH}^{A r 1}\right)$, 142.9 (br, $\left.\mathrm{C}^{P z 3,5}\right) \mathrm{ppm}$.

MS(ESI+) $: m / z(\%)=541(6)[\mathrm{M}-3 \mathrm{Cl}-2 \mathrm{H}]^{+}, 355(98)[\mathrm{M}-3 \mathrm{Cl}-2 \mathrm{H}-\mathrm{SA}]^{+}, 187(80)$ $[\mathrm{SA}+\mathrm{H}]^{+}$.

IR (KBr): 3424 (br, m), 3146 (m), 3105 (m), 3059 (s), 3012 (vs), 2949 (s), 2870 (s), $2783(\mathrm{~m}), 2425(\mathrm{w}), 1964(\mathrm{w}), 1775(\mathrm{w}), 1648(\mathrm{w}), 1607(\mathrm{~m}), 1550(\mathrm{~s}), 1486(\mathrm{~m})$, $1450(\mathrm{~m}), 1370(\mathrm{~m}), 1321(\mathrm{w}), 1262$ (w), $1203(\mathrm{~s}), 1163(\mathrm{~m}), 1107(\mathrm{w}), 1064(\mathrm{~m})$, $1034(\mathrm{w}), 968(\mathrm{w}), 896(\mathrm{w}), 860(\mathrm{~m}), 839(\mathrm{~m}), 755(\mathrm{~m}), 704(\mathrm{~m}), 669(\mathrm{w}), 627(\mathrm{~m})$, $579(\mathrm{w}), 536(\mathrm{w}), 446(\mathrm{w})$.

Schmelzpunkt: $309^{\circ} \mathrm{C}$. 
3,5-Bis[1-(4-nitrophenyl)imidazolium-1-ylmethyl]-pyrazol Hydrochlorid; $\left[\mathrm{H}_{4} \mathrm{~L}^{7}\right] \mathrm{Cl}_{3}$

Ausbeute: Diese wurde nicht bestimmt, da $\left[\mathrm{H}_{4} \mathrm{~L}^{7}\right] \mathrm{Cl}_{3}$ direkt weiter umgesetzt wurde; gelber Feststoff.

${ }^{1} \mathbf{H}-\mathbf{N M R}\left(500 \mathrm{MHz}, \mathrm{DMSO}-d_{6}\right): \delta=5.65\left(\mathrm{~s}, 4 \mathrm{H}, \mathrm{CH}_{2}\right), 7.33\left(\mathrm{~m}, 1 \mathrm{H}, \mathrm{CH}^{p-P h}\right)$, $7.38\left(\mathrm{~m}, 4 \mathrm{H}, \mathrm{CH}^{o, m-P h}\right), 7.95\left(\mathrm{t}, J=1.8 \mathrm{~Hz}, 2 \mathrm{H}, \mathrm{CH}^{I m}\right), 8.08\left(\mathrm{~m}, 4 \mathrm{H}, \mathrm{CH}^{A r 2,6}\right)$, $8.45\left(\mathrm{t}, J=1.8 \mathrm{~Hz}, 2 \mathrm{H}, \mathrm{CH}^{I m}\right), 8.47$ (m, 4H, $\left.\mathrm{CH}^{A r 3,5}\right), 10.35$ (s br, $2 \mathrm{H}, \mathrm{CH}^{I m 2}$ ).

${ }^{13}$ C-NMR (125 MHz, DMSO- $\left.d_{6}\right): \delta=44.6\left(\mathrm{CH}_{2}\right), 119.7\left(\mathrm{C}^{P z 4}\right), 123.0\left(\mathrm{CH}^{A r 2,6}\right)$, $123.1\left(\mathrm{CH}^{I m}\right), 123.9\left(\mathrm{br}, \mathrm{CH}^{I m}\right), 125.5$ (br, $\left.\mathrm{CH}^{A r 3,5}\right), 127.6\left(\mathrm{CH}^{p-P h}\right), 128.8$ $\left(\mathrm{CH}^{o-P h}\right), 129.5\left(\mathrm{CH}^{m-P h}\right), 130.2\left(\mathrm{C}^{i-P h}\right), 136.9,137.0\left(2 \times \mathrm{CH}^{\operatorname{Im} 2}\right), 139.1\left(\mathrm{C}^{A r 1}\right)$, $147.6\left(\mathrm{C}^{A r 4}\right) \mathrm{ppm}$.

IR (KBr): 3415 (br, m), 3160 (w), 3088 (w), 3017 (w), 2804 (w), 2715 (w), 2502 (w), $2403(\mathrm{w}), 1898(\mathrm{w}), 1724(\mathrm{w}), 1618(\mathrm{~m}), 1599(\mathrm{~m}), 1542(\mathrm{~s}), 1522(\mathrm{~s}), 1453(\mathrm{w})$, 1433 (w), 1342 (vs), 1303 (m), 1219 (m), 1106 (w), 1070 (m), 1011 (w), 955 (w), $893(\mathrm{w}), 852(\mathrm{~s}), 795(\mathrm{w}), 746(\mathrm{~m}), 704(\mathrm{w}), 681(\mathrm{w}), 623(\mathrm{w}), 532(\mathrm{w}), 502(\mathrm{w})$.

Schmelzpunkt: $187^{\circ} \mathrm{C}$.

\section{3,5-Bis[1-(1-phenylethyl)imidazolium-1-ylmethyl]-4-phenyl-pyrazol} Hydrochlorid; $\left[\mathrm{H}_{4} \mathrm{~L}^{10}\right] \mathrm{Cl}_{3}$

Ausbeute: $1.61 \mathrm{~g}(6.46 \mathrm{mmol}) \mathrm{Pz}^{\mathrm{H}}-\mathrm{THP} ; 1.97 \mathrm{~g}(3.61 \mathrm{mmol})\left[\mathrm{H}_{4} \mathrm{~L}^{10}\right] \mathrm{Cl}_{3} ; 56 \%$; hell gelber Feststoff.

${ }^{1} \mathbf{H}-\mathbf{N M R}\left(300 \mathrm{MHz}, \mathrm{CDCl}_{3}\right): \delta=1.92\left(\mathrm{~d}, J=6.4 \mathrm{~Hz}, 6 \mathrm{H}, \mathrm{CH}_{3}\right), 5.70(\mathrm{br} \mathrm{s}, 4 \mathrm{H}$, $\left.\mathrm{CH}_{2}\right), 5.77(\mathrm{~m}, 2 \mathrm{H}, \mathrm{CH}), 7.17\left(\mathrm{~s}, 2 \mathrm{H}, \mathrm{CH}^{I m}\right), 7.31\left(\mathrm{~m}, 10 \mathrm{H}, \mathrm{CH}^{P h}\right), 7.91$ (s, 2H, $\mathrm{CH}^{\operatorname{Im}}$ ), 10.41 (br s, $2 \mathrm{H}, \mathrm{CH}^{\operatorname{Im} 2}$ )ppm.

${ }^{13} \mathbf{C - N M R}\left(75 \mathrm{MHz}, \mathrm{CDCl}_{3}\right): \delta=21.2\left(\mathrm{CH}_{3}\right), 44.6\left(\mathrm{CH}_{2}\right), 60.0(\mathrm{CH}), 107.7\left(\mathrm{CH}^{P z}\right)$, 120.6, $123.3\left(2 \times \mathrm{CH}^{I m}\right), 126.9\left(\mathrm{CH}^{m-P h}\right), 129.3\left(\mathrm{CH}^{p-P h}\right), 129.4\left(\mathrm{CH}^{o-P h}\right), 136.2$ $\left(\mathrm{C}^{P z 3,5}\right), 137.9\left(\mathrm{CH}^{I m 2}\right), 141.0\left(\mathrm{C}^{P z 3,5}\right) \mathrm{ppm}$.

MS(ESI+): $m / z(\%)=437(5)[\mathrm{M}-3 \mathrm{Cl}-2 \mathrm{H}]^{+}, 333(100)[\mathrm{M}-3 \mathrm{Cl}-\mathrm{SA}-\mathrm{H}]^{+}, 105(95)$ $[\mathrm{SA}]^{+}$.

MS(ESI-): $m / z(\%)=545(10)[\mathrm{M}-\mathrm{H}]^{-}$. 


\section{3,5-Bis[1-(1-phenylethyl)imidazolium-1-ylmethyl]-4-phenyl-pyrazol}

\section{Hydrochlorid; $\left[\mathrm{H}_{4} \mathrm{~L}^{11}\right] \mathrm{Cl}_{3}$}

Ausbeute: $1.58 \mathrm{~g}$ (4.86mmol) $\mathrm{Pz}^{\mathrm{Ph}}$-THP; $1.95 \mathrm{~g}(3.13 \mathrm{mmol})\left[\mathrm{H}_{4} \mathrm{~L}^{11}\right] \mathrm{Cl}_{3} ; 64 \%$; hell gelber Feststoff.

${ }^{1} \mathbf{H}-\mathbf{N M R}\left(200 \mathrm{MHz}, \mathrm{CDCl}_{3}\right): \delta=1.82\left(\mathrm{~d}, J=6.6 \mathrm{~Hz}, 6 \mathrm{H}, \mathrm{CH}_{3}\right), 5.66(\mathrm{br} \mathrm{s}, 4 \mathrm{H}$, $\left.\mathrm{CH}_{2}\right), 5.80(\mathrm{q}, J=6.6 \mathrm{~Hz}, J=7.4 \mathrm{~Hz}, 2 \mathrm{H}, \mathrm{CH}), 7.09\left(\mathrm{br} \mathrm{s}, 2 \mathrm{H}, \mathrm{CH}^{I m}\right), 7.32(\mathrm{~m}, 15 \mathrm{H}$, $\mathrm{CH}^{P h}$ ), 7.39 (br s, 2H, $\mathrm{CH}^{I m}$ ), 10.31 (br s, 2H, $\mathrm{CH}^{I m 2}$ )ppm.

${ }^{13}$ C-NMR $\left(125 \mathrm{MHz}, \mathrm{CDCl}_{3}\right): \delta=21.0\left(\mathrm{CH}_{3}\right), 44.4\left(\mathrm{CH}_{2}\right), 59.5(\mathrm{CH}), 120.6$ $\left(\mathrm{CH}^{I m}\right), 121.1\left(\mathrm{C}^{P z 4}\right), 122.8\left(\mathrm{CH}^{I m}\right), 126.9\left(\mathrm{CH}^{m-P h}\right), 128.0\left(\mathrm{CH}^{p-P h}\right), 129.2$ $\left(\mathrm{CH}^{o-P h}\right), 129.3\left(\mathrm{C}^{i-P h}\right), 136.2\left(\mathrm{CH}^{I m 2}\right), 138.1\left(\mathrm{C}^{P z 3,5}\right) \mathrm{ppm}$.

MS(ESI+) $: m / z(\%)=513(7)[\mathrm{M}-3 \mathrm{Cl}-2 \mathrm{H}]^{+}, 409(72)[\mathrm{M}-3 \mathrm{Cl}-2 \mathrm{H}-\mathrm{SA}]^{+}, 105(49)$ $[\mathrm{SA}]^{+}$.

MS(ESI-): $m / z(\%)=621(18)[\mathrm{M}-\mathrm{H}]^{-}$.

IR (KBr): 3410 (br s), 3131 (m), 3084 (m), 2986 (m), 2064 (w), 1986 (w), 1902 (w), $1823(\mathrm{w}), 1628(\mathrm{~m}), 1556$ (m), $1495(\mathrm{w}), 1452(\mathrm{~m}), 1385(\mathrm{w}), 1329(\mathrm{w}), 1262$ (m), $1201(\mathrm{~m}), 1152(\mathrm{~s}), 1105(\mathrm{~m}), 1079(\mathrm{~m}), 1051(\mathrm{~m}), 1012(\mathrm{~m}), 973(\mathrm{w}), 923(\mathrm{w})$, $821(\mathrm{~m}), 758(\mathrm{~m}), 705(\mathrm{~s}), 648(\mathrm{~m}), 621(\mathrm{~m}), 536(\mathrm{~m})$.

\subsubsection{Umsalzen der Liganden}

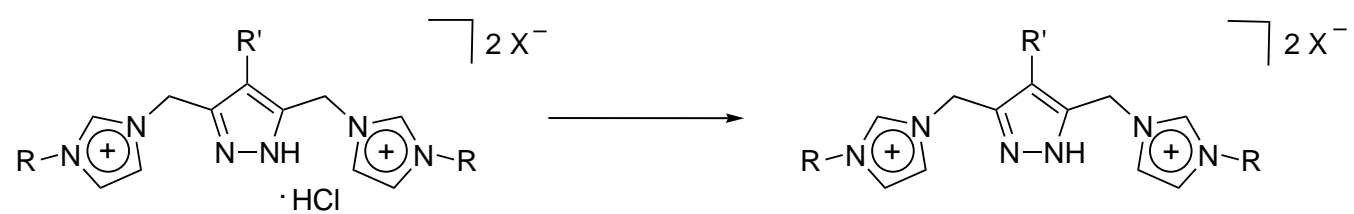

$$
\left.\begin{array}{c}
\mathrm{NH}_{4} \mathrm{PF}_{6} \\
\mathrm{H}_{2} \mathrm{O}
\end{array} \longrightarrow \begin{array}{l}
\mathrm{X}=\mathrm{Cl} \\
\mathrm{X}=\mathrm{BF}_{4} \\
\mathrm{X}=\mathrm{PF}_{6}
\end{array}\right] \begin{gathered}
\mathrm{NaBF}_{4} \\
\mathrm{H}_{2} \mathrm{O}
\end{gathered}
$$

Basierend auf [35]

Das entspechende Hydrochlorid (1.0Äq) wird in möglichst wenig Wasser gelöst. Ammoniak-Lösung (25\%, 3.0Äq) wird zugegeben und die Mischung wird für einige Minuten bei RT gerührt. Bei der Zugabe von Ammoniumhexafluorophosphat- 
bzw. Natriumtetrafluoroborat (2.2 ̈̈q) als Feststoff oder als konzentrierte wässrige Lösung, findet eine sofortige (weiße) Ausfällung statt. Das Reaktionsgemisch wird für weitere $30 \mathrm{~min}$ gerührt. Der entstandene Niederschlag wird abfiltriert, mit wenig Wasser gewaschen und im Vakuum getrocknet. Der Feststoff wird anschliessend in absolutem Acetonitril aufgenommen, um restliche Salze zu entfernen. Nach dem Abfiltrieren wird das Lösungsmittel im Hochvakuum entfernt, woraufhin das Produkt als beige-weißer Feststoff in meist guter Ausbeute erhalten werden kann.

\section{3,5-Bis[1-(tert-butyl)imidazolium-1-ylmethyl]-1H-pyrazol; $\left[\mathrm{H}_{3} \mathrm{~L}^{1}\right]\left(\mathrm{BF}_{4}\right)_{2}$}

Ausbeute: für $\left[\mathrm{H}_{3} \mathrm{~L}^{1}\right]\left(\mathrm{BF}_{4}\right)_{2}: 1.00 \mathrm{~g}(2.23 \mathrm{mmol})\left[\mathrm{H}_{4} \mathrm{~L}^{1}\right] \mathrm{Cl}_{3} ; 0.23 \mathrm{~g}(0.45 \mathrm{mmol})$ $\left[\mathrm{H}_{3} \mathrm{~L}^{1}\right]\left(\mathrm{BF}_{4}\right)_{2} ; 20 \%$. Für das entsprechende $\left[\mathrm{H}_{3} \mathrm{~L}^{1}\right]\left(\mathrm{PF}_{6}\right)_{2}: 5.20 \mathrm{~g}$ (11.60mmol) $\left[\mathrm{H}_{4} \mathrm{~L}^{1}\right] \mathrm{Cl}_{3} ; 6.61 \mathrm{~g}(10.45 \mathrm{mmol})\left[\mathrm{H}_{3} \mathrm{~L}^{1}\right]\left(\mathrm{PF}_{6}\right)_{2} ; 90 \%$. In beiden Fällen wurde das Produkt als weißer Feststoff erhalten. Durch Diethylether-Diffusion in eine MeOHLösung von $\left[\mathrm{H}_{3} \mathrm{~L}^{1}\right]\left(\mathrm{BF}_{4}\right)_{2}$ bei RT konnten farblose Kristalle gewonnen werden, die zur Röntgenkristallstrukturanalyse geeignet waren.

${ }^{1} \mathbf{H}-\mathrm{NMR}\left(500 \mathrm{MHz}, \mathrm{DMSO}-d_{6}\right): \delta=1.57(\mathrm{~s}, 9 \mathrm{H}, t \mathrm{Bu}), 1.58(\mathrm{~s}, 9 \mathrm{H}, t \mathrm{Bu}), 5.36$ $\left(\mathrm{s}, 2 \mathrm{H}, \mathrm{CH}_{2}\right), 5.46\left(\mathrm{~s}, 2 \mathrm{H}, \mathrm{CH}_{2}\right), 6.47\left(\mathrm{~d}, J=1.3 \mathrm{~Hz}, 1 \mathrm{H}, \mathrm{CH}^{P z}\right), 7.74(\mathrm{t}, J=1.6 \mathrm{~Hz}$, $\left.1 \mathrm{H}, \mathrm{CH}^{I m}\right), 7.76\left(\mathrm{t}, J=1.6 \mathrm{~Hz}, 1 \mathrm{H}, \mathrm{CH}^{I m}\right), 7.99\left(\mathrm{t}, J=1.6 \mathrm{~Hz}, 1 \mathrm{H}, \mathrm{CH}^{I m}\right), 8.03$ $\left(\mathrm{t}, J=1.6 \mathrm{~Hz}, 1 \mathrm{H}, \mathrm{CH}^{I m}\right), 9.30\left(\mathrm{t}, J=1.6 \mathrm{~Hz}, 1 \mathrm{H}, \mathrm{CH}^{I m 2}\right), 9.36(\mathrm{t}, J=1.6 \mathrm{~Hz}, 1 \mathrm{H}$, $\mathrm{CH}^{\operatorname{Im} 2}$ ), 13.18 (br s, $\left.1 \mathrm{H}, \mathrm{NH}\right) \mathrm{ppm}$.

${ }^{13}$ C-NMR $\left(125 \mathrm{MHz}, \mathrm{DMSO}-d_{6}\right): \delta=28.9,28.9(2 \times t \mathrm{Bu}), 43.1,46.1\left(2 \times \mathrm{CH}_{2}\right)$, 59.7, $59.8\left(2 \times \mathrm{C}^{t B u}\right), 104.9\left(\mathrm{CH}^{P z 4}\right), 120.4,120.8,122.5,122.8\left(4 \times \mathrm{CH}^{I m}\right), 134.6$, $134.8\left(2 \times \mathrm{CH}^{\operatorname{Im} 2}\right), 137.7\left(\mathrm{C}^{P z 5}\right), 146.1\left(\mathrm{C}^{P z 3}\right) \mathrm{ppm}$.

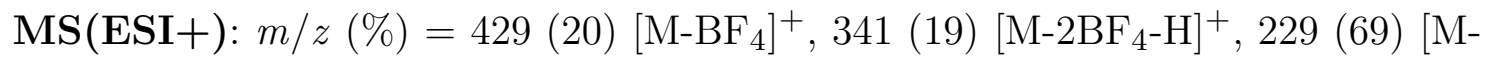
$\left.2 \mathrm{BF}_{4}-2 t \mathrm{Bu}-\mathrm{H}\right]^{+}, 161(100)\left[\mathrm{M}-2 \mathrm{BF}_{4}-2 t \mathrm{Bu}-\mathrm{Im}\right]^{+}, 69(40)[\mathrm{Im}+\mathrm{H}]^{+}$.

MS(ESI-): $m / z(\%)=87(100)\left[\mathrm{BF}_{4}\right]^{-}$.

IR (KBr): $\tilde{\nu}\left(\mathrm{cm}^{-1}\right)=3481(\mathrm{w}), 3072(\mathrm{w}), 3034(\mathrm{w}), 2977(\mathrm{w}), 2879(\mathrm{w}), 2775$ (w), $2624(\mathrm{w}), 2417(\mathrm{w}), 2313(\mathrm{w}), 2049(\mathrm{w}), 1630(\mathrm{w}), 1583(\mathrm{w}), 1555(\mathrm{~s}), 1469(\mathrm{w})$, 1448 (w), 1405 (w), 1379 (s), 1340 (w), 1306 (w), 1205 (s), 1127 (br, s), 1084 (br, s), $1034(\mathrm{br}, \mathrm{s}), 848(\mathrm{w}), 790(\mathrm{w}), 760(\mathrm{w}), 737(\mathrm{w}), 659(\mathrm{w}), 627(\mathrm{w}), 565(\mathrm{w}), 519$ $(\mathrm{w}), 522(\mathrm{w}), 492(\mathrm{w}), 445(\mathrm{w})$.

EA(\%): $\mathrm{C}_{19} \mathrm{H}_{30} \mathrm{~B}_{2} \mathrm{~F}_{8} \mathrm{~N}_{6}$ (516.09) berechnet: C 44.22, H 5.86, N 16.28; gefunden : $\quad$ C 43.98, H 5.88, N 16.48.

Schmelzpunkt: $203^{\circ} \mathrm{C}$. 
3,5-Bis[1-(tert-butyl)imidazolium-1-ylmethyl]-4-phenyl-1 $H$-pyrazol; $\left[\mathrm{H}_{3} \mathrm{~L}^{2}\right]\left(\mathrm{PF}_{6}\right)_{2}$

Ausbeute: $3.06 \mathrm{~g}$ (5.82mmol) $\left[\mathrm{H}_{4} \mathrm{~L}^{2}\right] \mathrm{Cl}_{3} ; 3.82 \mathrm{~g}(5.40 \mathrm{mmol})\left[\mathrm{H}_{3} \mathrm{~L}^{2}\right]\left(\mathrm{PF}_{6}\right)_{2} ; 93 \%$; weißer Feststoff.

${ }^{1} \mathbf{H}-\mathbf{N M R}\left(500 \mathrm{MHz}, \mathrm{DMSO}-d_{6}\right): \delta=1.46(\mathrm{~s}, 18 \mathrm{H}, t \mathrm{Bu}), 5.47\left(\mathrm{~s}, 4 \mathrm{H}, \mathrm{CH}_{2}\right), 7.18$ $\left(\mathrm{m}, 2 \mathrm{H}, \mathrm{CH}^{o-P h}\right), 7.36\left(\mathrm{~m}, 3 \mathrm{H}, \mathrm{CH}^{m, p-P h}\right), 7.53\left(\mathrm{t}, J=1.8 \mathrm{~Hz}, 2 \mathrm{H}, \mathrm{CH}^{I m}\right), 7.88$ (t, $J=1.8 \mathrm{~Hz}, 2 \mathrm{H}, \mathrm{CH}^{I m}$ ), 9.10 (br s, $2 \mathrm{H}, \mathrm{CH}^{I m 2}$ ), 13.44 (br s, $1 \mathrm{H}, \mathrm{NH}$ ) ppm.

${ }^{13}$ C-NMR $\left(125 \mathrm{MHz}, \mathrm{DMSO}-d_{6}\right): \delta=28.8(t \mathrm{Bu}), 43.0\left(\mathrm{br}, \mathrm{CH}_{2}\right), 45.2\left(\mathrm{br}, \mathrm{CH}_{2}\right)$, $59.6\left(\mathrm{C}^{t B u}\right), 119.0\left(\mathrm{C}^{P z 4}\right), 120.4\left(\mathrm{CH}^{I m}\right), 122.7\left(\mathrm{CH}^{I m}\right), 127.6\left(\mathrm{CH}^{p-P h}\right), 128.8$ $\left(\mathrm{CH}^{m-P h}\right), 129.1\left(\mathrm{CH}^{o-P h}\right), 130.2\left(\mathrm{C}^{i-P h}\right), 134.8\left(\mathrm{CH}^{I m 2}\right), 143.6\left(\mathrm{C}^{P z 3,5}\right) \mathrm{ppm}$.

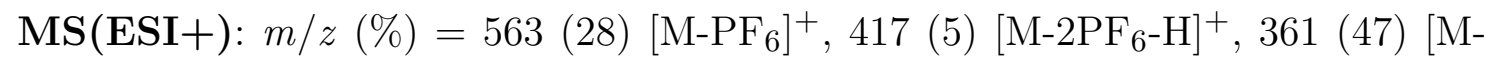
$\left.2 \mathrm{PF}_{6}-\mathrm{H}-\mathrm{Bu}\right]^{+}, 181(100)$.

MS(ESI-): $m / z(\%)=145(100)\left[\mathrm{PF}_{6}\right]^{-}$.

IR (KBr): $\tilde{\nu}\left(\mathrm{cm}^{-1}\right)=3401(\mathrm{w}), 3171(\mathrm{w}), 2987(\mathrm{w}), 2970(\mathrm{w}), 1613(\mathrm{w}), 1556$ (w), $1471(\mathrm{w}), 1450(\mathrm{w}), 1408(\mathrm{w}), 1383(\mathrm{w}), 1311(\mathrm{w}), 1261(\mathrm{w}), 1203(\mathrm{~m}), 1131$ (m), $1111(\mathrm{w}), 838$ (br, s), $740(\mathrm{~m}), 704(\mathrm{~m}), 658(\mathrm{~m}), 625(\mathrm{w}), 558(\mathrm{~s}), 471(\mathrm{w})$.

Schmelzpunkt: $151^{\circ} \mathrm{C}$.

3,5-Bis[1-(1-adamantyl)imidazolium-1-ylmethyl]-1 $H$-pyrazol; $\left[\mathrm{H}_{3} \mathrm{~L}^{3}\right]\left(\mathrm{BF}_{4}\right)_{2}$

Ausbeute: für $\left[\mathrm{H}_{3} \mathrm{~L}^{3}\right]\left(\mathrm{BF}_{4}\right)_{2}: 1.40 \mathrm{~g}(2.31 \mathrm{mmol})\left[\mathrm{H}_{4} \mathrm{~L}^{3}\right] \mathrm{Cl}_{3} ; 1.06 \mathrm{~g}(1.57 \mathrm{mmol})$ $\left[\mathrm{H}_{3} \mathrm{~L}^{3}\right]\left(\mathrm{BF}_{4}\right)_{2} ; 68 \%$. Für das entsprechende $\left[\mathrm{H}_{3} \mathrm{~L}^{3}\right]\left(\mathrm{PF}_{6}\right)_{2}: 7.24 \mathrm{~g}$ (11.94mmol) $\left[\mathrm{H}_{4} \mathrm{~L}^{3}\right] \mathrm{Cl}_{3} ; 7.03 \mathrm{~g}(8.91 \mathrm{mmol})\left[\mathrm{H}_{3} \mathrm{~L}^{3}\right]\left(\mathrm{PF}_{6}\right)_{2} ; 75 \%$. In beiden Fällen wurde das Produkt als weißer Feststoff erhalten. Durch Diethylether-Diffusion in eine AcetonLösung von $\left[\mathrm{H}_{3} \mathrm{~L}^{3}\right]\left(\mathrm{BF}_{4}\right)_{2}$ bei RT konnten farblose Kristalle gewonnen werden, die zur Röntgenkristallstrukturanalyse geeignet waren.

${ }^{1}$ H-NMR $\left(500 \mathrm{MHz}\right.$, DMSO- $\left.d_{6}\right): \delta=1.71\left(\mathrm{~m}, 12 \mathrm{H}, \mathrm{CH}_{2}^{\mathrm{Ad}}\right), 2.10\left(\mathrm{~m}, 12 \mathrm{H}, \mathrm{CH}_{2}^{\mathrm{Ad}}\right)$, $2.21\left(\mathrm{~m}, 6 \mathrm{H}, \mathrm{CH}^{A d}\right), 5.36\left(\mathrm{~s}, 2 \mathrm{H}, \mathrm{CH}_{2}\right), 5.46\left(\mathrm{~s}, 2 \mathrm{H}, \mathrm{CH}_{2}\right), 6.46\left(\mathrm{~s}, 1 \mathrm{H}, \mathrm{CH}^{P z}\right), 7.77$ $\left(\mathrm{s}, 2 \mathrm{H}, \mathrm{CH}^{I m}\right), 8.03\left(\mathrm{~s}, 1 \mathrm{H}, \mathrm{CH}^{\operatorname{Im}}\right), 8.06\left(\mathrm{~s}, 1 \mathrm{H}, \mathrm{CH}^{I m}\right), 9.35\left(\mathrm{~s}, 1 \mathrm{H}, \mathrm{CH}^{\operatorname{Im} 2}\right), 9.38$ $\left(\mathrm{s}, 1 \mathrm{H}, \mathrm{CH}^{\operatorname{Im} 2}\right), 13.23$ (br s, $\left.1 \mathrm{H}, \mathrm{NH}\right) \mathrm{ppm}$.

${ }^{13}$ C-NMR $\left(125 \mathrm{MHz}, \mathrm{DMSO}-d_{6}\right): \delta=28.8\left(\mathrm{CH}^{A d}\right), 34.8\left(\mathrm{CH}_{-} \mathrm{CH}_{2}-\mathrm{CH}^{A d}\right), 41.5$, $41.5\left(2 \times \mathrm{C}^{-} \mathrm{CH}_{2}^{A d}\right), 43.1,46.2\left(2 \times \mathrm{CH}_{2}\right), 59.3,59.4\left(2 \times \mathrm{C}^{A d}\right), 104.9\left(\mathrm{CH}^{P z 4}\right)$, 
119.5, 119.8, 122.5, $122.8\left(4 \times \mathrm{CH}^{I m}\right), 134.1,134.4\left(2 \times \mathrm{CH}^{\operatorname{Im} 2}\right), 137.6\left(\mathrm{CH}^{P z 5}\right)$, $146.1\left(\mathrm{CH}^{P z 3}\right) \mathrm{ppm}$.

$\operatorname{MS}(\mathbf{E S I}+): m / z(\%)=585(18)\left[\mathrm{M}-B F_{4}\right]^{+}, 363(100)\left[\mathrm{M}-2 \mathrm{BF}_{4}-\mathrm{Ad}\right]^{+}, 135(85)$ $[\mathrm{Ad}]^{+}$.

MS(ESI-): $m / z(\%)=87(100)\left[\mathrm{BF}_{4}\right]^{-}$.

IR (KBr): $\tilde{\nu}\left(\mathrm{cm}^{-1}\right)=3415(\mathrm{~s}), 3167(\mathrm{~m}), 2916(\mathrm{~s}), 2859(\mathrm{~m}), 1627(\mathrm{w}), 1553(\mathrm{~m})$, $1454(\mathrm{~m}), 1366$ (w), 1348 (w), 1310 (m), 1259 (w), 1192 (w), 1151 (s), 1106 (m), $1067(\mathrm{w}), 1032(\mathrm{w}), 1000(\mathrm{w}), 839(\mathrm{br}, \mathrm{vs}), 741(\mathrm{~m}), 659(\mathrm{w}), 638(\mathrm{w}), 619(\mathrm{w}), 557$ (vs).

$\mathbf{E A}(\%): \mathrm{C}_{31} \mathrm{H}_{42} \mathrm{~B}_{2} \mathrm{~F}_{8} \mathrm{~N}_{6}$ (672.31) berechnet: C 55.38, H 6.29, N 12.50; gefunden : $\quad$ C 54.91, H 6.18, N 12.52 .

Schmelzpunkt: $233^{\circ} \mathrm{C}$.

3,5-Bis[1-(1-adamantyl)imidazolium-1-ylmethyl]-4-phenyl-1 $H$-pyrazol; $\left[\mathrm{H}_{3} \mathrm{~L}^{4}\right]\left(\mathrm{PF}_{6}\right)_{2}$

Ausbeute: $\quad 0.62 \mathrm{~g} \quad(0.91 \mathrm{mmol}) \quad\left[\mathrm{H}_{4} \mathrm{~L}^{4}\right] \mathrm{Cl}_{3} ; \quad 0.66 \mathrm{~g} \quad(0.76 \mathrm{mmol}) \quad\left[\mathrm{H}_{3} \mathrm{~L}^{4}\right]\left(\mathrm{PF}_{6}\right)_{2}$; $84 \%$; weißer Feststoff. Durch Diethylether-Diffusion in eine Aceton/MeOHMischung/Lösung (1:1) des Rohproduktes bei $+8^{\circ} \mathrm{C}$ konnten farblose Kristalle gewonnen werden, die zur Röntgenkristallstrukturanalyse geeignet waren.

${ }^{1} \mathbf{H}-\mathbf{N M R}\left(500 \mathrm{MHz}, \mathrm{DMSO}-d_{6}\right): \delta=1.67\left(\mathrm{~m}, 12 \mathrm{H}, \mathrm{CH}_{2}^{A d}\right), 1.95(\mathrm{~d}, J=2.5 \mathrm{~Hz}$, $\left.12 \mathrm{H}, \mathrm{CH}_{2}^{A d}\right), 2.18\left(\mathrm{~m}, 6 \mathrm{H}, \mathrm{CH}^{A d}\right), 5.47\left(\mathrm{~s}, 4 \mathrm{H}, \mathrm{CH}_{2}\right), 7.17\left(\mathrm{~m}, 2 \mathrm{H}, \mathrm{CH}^{o-P h}\right), 7.37$ $\left(\mathrm{m}, 3 \mathrm{H}, \mathrm{CH}^{m, p-P h}\right), 7.53\left(\mathrm{t}, J=1.8 \mathrm{~Hz}, 2 \mathrm{H}, \mathrm{CH}^{I m}\right), 7.92\left(\mathrm{t}, J=1.8 \mathrm{~Hz}, 2 \mathrm{H}, \mathrm{CH}^{I m}\right)$, 9.10 (t, J=1.6 Hz, 2H, $\mathrm{CH}^{\operatorname{Im} 2}$ ), 13.55 (br s, $1 \mathrm{H}, \mathrm{NH}$ ) ppm.

${ }^{13} \mathbf{C}-\mathrm{NMR}\left(125 \mathrm{MHz}, \mathrm{DMSO}-d_{6}\right): \delta=28.8\left(\mathrm{CH}^{A d}\right), 28.9\left(\mathrm{CH}-\mathrm{CH}_{2}-\mathrm{CH}^{A d}\right), 41.4$ $\left(\mathrm{C}_{-} \mathrm{CH}_{2}^{A d}\right), 44.2\left(\mathrm{br}, \mathrm{CH}_{2}\right), 59.2\left(\mathrm{C}^{A d}\right), 119.0\left(\mathrm{C}^{P z 4}\right), 119.5,122.7\left(2 \mathrm{x} \mathrm{CH}^{I m}\right), 127.6$ $\left(\mathrm{CH}^{p-P h}\right), 128.8\left(\mathrm{CH}^{m-P h}\right), 129.0\left(\mathrm{CH}^{o-P h}\right), 130.3\left(\mathrm{C}^{i-P h}\right), 134.3\left(\mathrm{CH}^{I m 2}\right) \mathrm{ppm}$.

$\operatorname{MS}(\mathbf{E S I}+): m / z(\%)=719(18)\left[{\mathrm{M}-\mathrm{PF}_{6}}^{+}\right]^{+}, 440(70)\left[\mathrm{M}-2 \mathrm{PF}_{6}-\mathrm{Ad}+\mathrm{H}\right]^{+}, 135(100)$ $[\mathrm{Ad}]^{+}$.

IR (KBr): $\tilde{\nu}\left(\mathrm{cm}^{-1}\right)=3460(\mathrm{br}, \mathrm{m}), 3153(\mathrm{w}), 3078(\mathrm{w}), 2911(\mathrm{~m}), 2856(\mathrm{~m}), 2683$ (w), $1609(\mathrm{w}), 1552(\mathrm{~s}), 1452(\mathrm{~s}), 1364(\mathrm{~m}), 1347(\mathrm{w}), 1309(\mathrm{~m}), 1258(\mathrm{w}), 1155(\mathrm{~s})$, 1072 (br, s), 1032 (br, s), $816(\mathrm{~m}), 755(\mathrm{~m}), 704(\mathrm{~m}), 659(\mathrm{w}), 625(\mathrm{w}), 521(\mathrm{w}), 526$ (w), $471(\mathrm{w})$. 
EA(\%): $\mathrm{C}_{74} \mathrm{H}_{91} \mathrm{~F}_{18} \mathrm{~N}_{12} \mathrm{P}_{3}, 0.5 \mathrm{CH}_{3} \mathrm{CN}, 0.5 \mathrm{H}_{2} \mathrm{O}(1613.02)$
berechnet:
C 55.84, H 5.84, N 10.85;
gefunden :
C 55.80, H 6.03, N 10.87.

Schmelzpunkt: $209^{\circ} \mathrm{C}$.

\section{3,5-Bis[1-(2,4,6-trimethylphenyl)imidazolium-1-ylmethyl]-4-phenyl-1 H- pyrazol; $\left[\mathrm{H}_{3} \mathrm{~L}^{6}\right]\left(\mathrm{BF}_{4}\right)_{2}$}

Ausbeute: $0.44 \mathrm{~g}(0.71 \mathrm{mmol})\left[\mathrm{H}_{4} \mathrm{~L}^{6}\right] \mathrm{Cl}_{3} ; 0.27 \mathrm{~g}(0.38 \mathrm{mmol})\left[\mathrm{H}_{3} \mathrm{~L}^{6}\right]\left(\mathrm{BF}_{4}\right)_{2} ; 53 \%$. Für die entsprechende Hexafluorophosphat-Verbindung $\left[\mathrm{H}_{3} \mathrm{~L}^{6}\right]\left(\mathrm{PF}_{6}\right)_{2}: 0.86 \mathrm{~g}$ (1.33 mmol) $\left[\mathrm{H}_{4} \mathrm{~L}^{6}\right] \mathrm{Cl}_{3} ; 1.02 \mathrm{~g}(1.23 \mathrm{mmol})\left[\mathrm{H}_{3} \mathrm{~L}^{6}\right]\left(\mathrm{PF}_{6}\right)_{2} ; 92 \%$. In beiden Fällen wurde das Produkt als weißer Feststoff erhalten. Durch Diethylether-Diffusion in eine MeCN-Lösung von $\left[\mathrm{H}_{3} \mathrm{~L}^{6}\right]\left(\mathrm{BF}_{4}\right)_{2}$ bei RT konnten farblose Kristalle gewonnen werden, die zur Röntgenkristallstrukturanalyse geeignet waren.

${ }^{1} \mathrm{H}-\mathrm{NMR}\left(500 \mathrm{MHz}, \mathrm{DMSO}-d_{6}\right): \delta=1.87,1.90\left(2 \mathrm{x} \mathrm{s}, 6 \mathrm{H}, \mathrm{CH}_{3}{ }^{A r 2,6}\right), 2.31(\mathrm{~s}, 6 \mathrm{H}$, $\left.\mathrm{CH}_{3}{ }^{A r 4}\right), 5.64,5.67\left(2 \times \mathrm{s}, 2 \mathrm{H}, \mathrm{CH}_{2}\right), 7.10,7.11\left(2 \mathrm{x} \mathrm{s}, 2 \mathrm{H}, \mathrm{CH}^{A r 3,5}\right), 7.33(\mathrm{~m}, 3 \mathrm{H}$, $\left.\mathrm{CH}^{m, p-P h}\right), 7.40\left(\mathrm{~m}, 2 \mathrm{H}, \mathrm{CH}^{o-P h}\right), 7.82$ (br s, $\left.1 \mathrm{H}, \mathrm{CH}^{I m}\right), 7.86(\mathrm{t}, J=1.6 \mathrm{~Hz}, 3 \mathrm{H}$, $\left.\mathrm{CH}^{I m}\right), 9.31,9.34\left(2 \times \mathrm{s}, 1 \mathrm{H}, \mathrm{CH}^{\operatorname{Im} 2}\right), 13.74$ (s, $\left.1 \mathrm{H}, \mathrm{NH}\right) \mathrm{ppm}$.

${ }^{13}$ C-NMR $\left(125 \mathrm{MHz}, \mathrm{DMSO}-d_{6}\right): \delta=16.6,16.7\left(2 \times \mathrm{CH}_{3}{ }^{A r 2,6}\right), 20.4\left(\mathrm{CH}_{3}{ }^{A r 4}\right)$, 43.0, $45.9\left(2 \times \mathrm{CH}_{2}\right), 119.4\left(\mathrm{C}^{P z 4}\right), 122.9,123.5,123.7,124.2\left(4 \times \mathrm{CH}^{I m}\right), 127.6$ $\left(\mathrm{CH}^{p-P h}\right), 128.6\left(\mathrm{CH}^{o-P h}\right), 128.9\left(\mathrm{CH}^{m-P h}\right), 129.0\left(\mathrm{CH}^{A r 3,5}\right), 130.0\left(\mathrm{C}^{i-P h}\right), 130.8$ $\left(\mathrm{C}^{A r 1}\right), 133.6\left(\mathrm{C}^{P z 3,5}\right), 134.1\left(\mathrm{C}^{A r 2,6}\right), 137.3,137.5\left(2 \times \mathrm{CH}^{\operatorname{Im} 2}\right), 140.2\left(\mathrm{C}^{A r 4}\right), 143.2$ $\left(\mathrm{C}^{P z 3,5}\right) \mathrm{ppm}$.

MS(ESI+) $: m / z(\%)=629(5)\left[\mathrm{M}-B F_{4}\right]^{+}, 541(2)\left[\mathrm{M}-2 \mathrm{BF}_{4}-\mathrm{H}\right]^{+}, 355$ (45) [M$\left.2 \mathrm{BF}_{4}-\mathrm{H}-\mathrm{SA}\right]^{+}, 271(100)\left[\mathrm{M}-2 \mathrm{BF}_{4}\right]^{2}+, 187(60)[\mathrm{SA}+\mathrm{H}]^{+}$.

MS(ESI-): $m / z(\%)=87(100)\left[\mathrm{BF}_{4}\right]^{-}$.

IR (KBr): $\tilde{\nu}\left(\mathrm{cm}^{-1}\right)=3411(\mathrm{br}, \mathrm{m}), 3151(\mathrm{~m}), 3123(\mathrm{~m}), 3073(\mathrm{~m}), 2968(\mathrm{~m})$, 1763 (w), 1606 (m), 1560 (m), 1487 (w), $1444(\mathrm{~m}), 1377$ (w), 1292 (w), 1262 (w), $1203(\mathrm{~m}), 1158(\mathrm{~m}), 1078(\mathrm{~s}), 1034(\mathrm{~s}), 966(\mathrm{w}), 932(\mathrm{w}), 870(\mathrm{w}), 839(\mathrm{w}), 803(\mathrm{w})$, $755(\mathrm{~m}), 703(\mathrm{w}), 673(\mathrm{w}), 627(\mathrm{w}), 581(\mathrm{w}), 522(\mathrm{w}), 448(\mathrm{w})$.

EA(\%): $\mathrm{C}_{35} \mathrm{H}_{38} \mathrm{~B}_{2} \mathrm{~F}_{8} \mathrm{~N}_{6}$ (716.32) berechnet: $\quad$ C 58.63, H 5.34, N 11.73;

gefunden : $\quad$ C 58.27, H 5.19, N 11.66.

Schmelzpunkt: $280^{\circ} \mathrm{C}$. 
3,5-Bis[1-(4-nitrophenyl)imidazolium-1-ylmethyl]-4-phenyl-1 $H$-pyrazol; $\left[\mathrm{H}_{3} \mathrm{~L}^{7}\right]\left(\mathrm{PF}_{6}\right)_{2}$

Ausbeute: $0.12 \mathrm{~g}(0.19 \mathrm{mmol})\left[\mathrm{H}_{4} \mathrm{~L}^{7}\right] \mathrm{Cl}_{3} ; 0.08 \mathrm{~g}(0.09 \mathrm{mmol})\left[\mathrm{H}_{3} \mathrm{~L}^{7}\right]\left(\mathrm{PF}_{6}\right)_{2} ; 50 \%$; gelber Feststoff. Durch Diethylether-Diffusion in eine MeOH-Lösung des Rohproduktes bei RT konnten farblose Kristalle gewonnen werden, die zur Röntgenkristallstrukturanalyse geeignet waren.

${ }^{1} \mathbf{H}-\mathrm{NMR}\left(500 \mathrm{MHz}, \mathrm{DMSO}-d_{6}\right): \delta=5.57,5.62\left(2 \mathrm{x} \mathrm{s}, 2 \mathrm{H}, \mathrm{CH}_{2}\right), 7.32(\mathrm{~m}, 3 \mathrm{H}$, $\left.\mathrm{CH}^{m, p-P h}\right), 7.41\left(\mathrm{~m}, 2 \mathrm{H}, \mathrm{CH}^{o-P h}\right), 7.85\left(\mathrm{~s}, 2 \mathrm{H}, \mathrm{CH}^{I m}\right), 7.97\left(\mathrm{~m}, 4 \mathrm{H}, \mathrm{CH}^{A r 2,6}\right)$, $8.38\left(\mathrm{~s}, 2 \mathrm{H}, \mathrm{CH}^{\operatorname{Im}}\right), 8.52$ (br s, $\left.4 \mathrm{H}, \mathrm{CH}^{A r 3,5}\right), 9.91,9.95\left(2 \mathrm{x} \mathrm{s}, 2 \mathrm{H}, \mathrm{CH}^{\operatorname{Im} 2}\right), 13.50$ (br s, $1 \mathrm{H}, \mathrm{NH}) \mathrm{ppm}$.

${ }^{13}$ C-NMR (125MHz, DMSO- $\left.d_{6}\right): \delta=43.6\left(\mathrm{CH}_{2}\right), 119.8\left(\mathrm{C}^{P z 4}\right), 121.4$ (br, $\left.\mathrm{CH}^{A r 2,6}\right), 123.0,125.6$ (2 x $\left.\mathrm{CH}^{I m}\right), 124.0$ (br, $\left.\mathrm{CH}^{A r 3,5}\right), 127.8\left(\mathrm{CH}^{p-P h}\right), 128.9$ $\left(\mathrm{CH}^{m-P h}\right), 129.3\left(\mathrm{CH}^{o-P h}\right), 130.0\left(\mathrm{C}^{i-P h}\right), 134.5\left(\mathrm{br}, \mathrm{C}^{A r 1}\right), 136.6\left(\mathrm{br}, \mathrm{CH}^{I m 2}\right)$, $138.9\left(\mathrm{CH}^{P z 3,5}\right), 143.0\left(\mathrm{C}^{A r 4}\right), 147.7\left(\mathrm{CH}^{P z 3,5}\right) \mathrm{ppm}$.

MS(ESI+): $m / z(\%)=693(12)\left[\mathrm{M}-\mathrm{PF}_{6}\right]^{+}, 547(3)\left[\mathrm{M}-2 \mathrm{PF}_{6}-\mathrm{H}\right]^{+}, 358(46)[\mathrm{M}-$ $\left.2 \mathrm{PF}_{6}-\mathrm{SA}\right]^{+}, 190(51)[\mathrm{SA}+\mathrm{H}]^{+}$.

MS(ESI-): $m / z(\%)=145(100)\left[\mathrm{PF}_{6}\right]^{-}$.

IR (KBr): $\tilde{\nu}\left(\mathrm{cm}^{-1}\right)=3407(\mathrm{br}, \mathrm{w}), 3160(\mathrm{w}), 2964(\mathrm{w}), 1620(\mathrm{w}), 1601(\mathrm{w}), 1553$ (m), 1530 (m), 1445 (w), $1346(\mathrm{~m}), 1262$ (w), 1205 (w), 1107 (w), $1074(\mathrm{~m}), 1014$ $(\mathrm{w}), 959(\mathrm{w}), 842(\mathrm{~s}), 748(\mathrm{~m}), 706(\mathrm{w}), 689(\mathrm{w}), 622(\mathrm{w}), 558(\mathrm{~m})$.

EA(\%): $\mathrm{C}_{29} \mathrm{H}_{24} \mathrm{~F}_{12} \mathrm{~N}_{8} \mathrm{O}_{4} \mathrm{P}_{2}$ (838.48) berechnet: C 41.54, H 2.88, N 13.36; gefunden : $\quad$ C 41.10, H 3.06, N 13.27.

Schmelzpunkt: $182^{\circ} \mathrm{C}$.

3,5-Bis[1-benzylimidazolium-1-ylmethyl]-4-phenyl-1 $H$-pyrazol; $\left[\mathrm{H}_{3} \mathrm{~L}^{9}\right]\left(\mathrm{BF}_{4}\right)_{2}$

Ausbeute: $2.57 \mathrm{~g}(3.50 \mathrm{mmol})\left[\mathrm{H}_{4} \mathrm{~L}^{9}\right] \mathrm{Cl}_{3} ; 2.11 \mathrm{~g}(3.19 \mathrm{mmol})\left[\mathrm{H}_{3} \mathrm{~L}^{9}\right]\left(\mathrm{BF}_{4}\right)_{2} ; 91 \%$; beiger Feststoff.

$\operatorname{MS}(\mathbf{E S I}+): m / z(\%)=573(15)\left[\mathrm{M}-B F_{4}\right]^{+}, 497(7)\left[\mathrm{M}-\mathrm{BF}_{4}-\mathrm{Ph}\right]^{+}, 395(62)[\mathrm{M}-$ $\left.2 \mathrm{BF}_{4}-\mathrm{CH}_{2} \mathrm{Ph}\right]^{+}, 327(100)\left[\mathrm{M}-2 \mathrm{BF}_{4}-\mathrm{SA}\right]^{+}, 91(88)\left[\mathrm{CH}_{2} \mathrm{Ph}\right]^{+}$.

MS(ESI-): $m / z(\%)=87(100)\left[\mathrm{BF}_{4}\right]^{-}$. 
IR (KBr): $\tilde{\nu}\left(\mathrm{cm}^{-1}\right)=3414(\mathrm{br}, \mathrm{s}), 3132(\mathrm{~s}), 3082(\mathrm{~s}), 2409(\mathrm{w}), 2070(\mathrm{w}), 1975$ (w), $1900(\mathrm{w}), 1825(\mathrm{w}), 1723(\mathrm{w}), 1608(\mathrm{~m}), 1613(\mathrm{~m}), 1560$ (s), $1498(\mathrm{~m}), 1452$ (m), 1404 (m), 1358 (m), 1304 (w), 1152 (s), 1080 (s), 1039 (s), 822 (m), 751 (m), $707(\mathrm{~s}), 620(\mathrm{~m}), 574(\mathrm{w}), 522(\mathrm{~m}), 463(\mathrm{w})$.

\section{3,5-Bis[1-(1-phenylethyl)imidazolium-1-ylmethyl]-1 $H$-pyrazol;} $\left[\mathrm{H}_{3} \mathrm{~L}^{10}\right]\left(\mathrm{BF}_{4}\right)_{2}$

Ausbeute: $4.81 \mathrm{~g}(8.81 \mathrm{mmol})\left[\mathrm{H}_{4} \mathrm{~L}^{10}\right] \mathrm{Cl}_{3} ; 3.88 \mathrm{~g}(6.34 \mathrm{mmol})\left[\mathrm{H}_{3} \mathrm{~L}^{10}\right]\left(\mathrm{BF}_{4}\right)_{2} ; 72 \%$; beiger Feststoff.

${ }^{1} \mathbf{H}-\mathbf{N M R}\left(500 \mathrm{MHz}, \mathrm{DMSO}-d_{6}\right): \delta=1.88\left(\mathrm{~d}, J=7.1 \mathrm{~Hz}, 6 \mathrm{H}, \mathrm{CH}_{3}\right), 5.40,5.49(2 \mathrm{x}$ s, $2 \mathrm{H}, \mathrm{CH}_{2}$ ), 5.81 (q, J=7.0 Hz, $\left.2 \mathrm{H}, \mathrm{CH}\right), 6.48\left(\mathrm{~s}, 1 \mathrm{H}, \mathrm{CH}^{P z}\right), 7.43\left(\mathrm{~m}, 10 \mathrm{H}, \mathrm{CH}^{P h}\right)$, 7.75 (d, J=5.3 Hz, 2H, CH $\left.{ }^{I m}\right), 7.91$ (br s, $\left.2 \mathrm{H}, \mathrm{CH}^{I m}\right), 9.38,9.44\left(2 \mathrm{x} \mathrm{CH}^{I m 2}\right), 13.28$ (br s, $1 \mathrm{H}, \mathrm{NH}) \mathrm{ppm}$.

${ }^{13}$ C-NMR $\left(125 \mathrm{MHz}, \mathrm{DMSO}-d_{6}\right): \delta=20.4\left(\mathrm{CH}_{3}\right), 43.2,46.2\left(2 \times \mathrm{CH}_{2}\right), 58.7$, $58.8(2 \times \mathrm{CH}), 105.1\left(\mathrm{CH}^{P z}\right), 121.2,121.6,122.8,123.1\left(\mathrm{CH}^{I m}\right), 125.5,126.6$ $\left(\mathrm{CH}^{p-P h}\right), 128.8\left(\mathrm{C}^{i-P h}\right), 129.0\left(\mathrm{CH}^{o, m-P h}\right), 135.5,135.7,139.2,139.4\left(\mathrm{CH}^{I m 2}\right)$, $146.0\left(\mathrm{C}^{P z 3,5}\right) \mathrm{ppm}$.

MS(ESI+): $m / z(\%)=525(35)\left[\mathrm{M}-B F_{4}\right]^{+}, 437(5)\left[\mathrm{M}-2 \mathrm{BF}_{4}-\mathrm{H}\right]^{+}, 333(100)[\mathrm{M}-$ $\left.2 \mathrm{BF}_{4}-\mathrm{SA}-\mathrm{H}\right]^{+}, 105(70)[\mathrm{SA}]^{+}$.

MS(ESI-): $m / z(\%)=87(100)\left[\mathrm{BF}_{4}\right]^{-}$.

IR (KBr): $\tilde{\nu}\left(\mathrm{cm}^{-1}\right)=3471(\mathrm{~m}), 3412(\mathrm{~m}), 3153(\mathrm{w}), 3121(\mathrm{w}), 3065(\mathrm{w}), 2985$ (w), $2878(\mathrm{w}), 1634$ (w), 1558 (m), $1498(\mathrm{w}), 1450(\mathrm{w}), 1384(\mathrm{w}), 1350(\mathrm{w}), 1298$ (w), $1262(\mathrm{w}), 1162(\mathrm{~m}), 1082(\mathrm{~s}), 1035(\mathrm{~s}), 922(\mathrm{w}), 841(\mathrm{w}), 794(\mathrm{w}), 767(\mathrm{~m}), 702$ (m), $640(\mathrm{w}), 584(\mathrm{w}), 522(\mathrm{w}), 462(\mathrm{w})$.

EA(\%): $\mathrm{C}_{27} \mathrm{H}_{30} \mathrm{~B}_{2} \mathrm{~F}_{8} \mathrm{~N}_{6}$ (612.18) berechnet: C 52.97, H 4.94, N 13.73;

gefunden : $\quad$ C 52.60, H 4.68, N 13.92 .

Schmelzpunkt: $210^{\circ} \mathrm{C}$. 
3,5-Bis[1-(1-phenylethyl)imidazolium-1-ylmethyl]-4-phenyl-1 $H$-pyrazol; $\left[\mathrm{H}_{3} \mathrm{~L}^{11}\right]\left(\mathrm{BF}_{4}\right)_{2}$

Ausbeute: $0.89 \mathrm{~g}(1.43 \mathrm{mmol})\left[\mathrm{H}_{4} \mathrm{~L}^{11}\right] \mathrm{Cl}_{3} ; 0.94 \mathrm{~g}(1.36 \mathrm{mmol})\left[\mathrm{H}_{3} \mathrm{~L}^{11}\right]\left(\mathrm{BF}_{4}\right)_{2} ; 95 \%$; weißer Feststoff. Durch Diethylether-Diffusion in eine MeCN-Lösung des Rohproduktes bei RT konnten farblose Kristalle erhalten werden, die zur Röntgenkristallstrukturanalyse geeignet waren.

${ }^{1} \mathbf{H}-\mathrm{NMR}\left(500 \mathrm{MHz}, \mathrm{DMSO}-d_{6}\right): \delta=1.78\left(\mathrm{t}, J=4.8 \mathrm{~Hz}, 6 \mathrm{H}, \mathrm{CH}_{3}\right), 5.46,5.50(2$ x s, $2 \mathrm{H}, \mathrm{CH}_{2}$ ), 5.70 (q, J=7.2 Hz, 2H, CH), $7.13\left(\mathrm{~m}, 2 \mathrm{H}, \mathrm{CH}^{o-P h}\right), 7.33(\mathrm{~m}, 13 \mathrm{H}$, $\left.\mathrm{CH}^{P h}\right), 7.53$ (br s, $2 \mathrm{H}, \mathrm{CH}^{I m}$ ), 7.80 (br s, $\left.2 \mathrm{H}, \mathrm{CH}^{I m}\right), 9.23$ (br s, $2 \mathrm{H}, \mathrm{CH}^{I m}$ ), 13.49 (br s, $1 \mathrm{H}, \mathrm{NH}) \mathrm{ppm}$.

${ }^{13}$ C-NMR (125MHz, DMSO- $\left.d_{6}\right): \delta=20.4\left(\mathrm{CH}_{3}\right), 42.8,45.5\left(2 \times \mathrm{CH}_{2}\right), 58.6,58.7$ (2 x CH), $119.3\left(\mathrm{C}^{P z 4}\right), 121.0,121.4,122.8,123.2\left(\mathrm{CH}^{I m}\right), 126.4,126.5\left(\mathrm{CH}^{o-P h}\right)$, 128.7, $128.8\left(\mathrm{CH}^{p-P h}\right), 128.9\left(\mathrm{CH}^{m-P h}\right), 130.0\left(\mathrm{C}^{i-P h}\right), 139.0,139.2\left(2 \times \mathrm{CH}^{I m 2}\right)$, $143.5\left(\mathrm{C}^{P z 3,5}\right) \mathrm{ppm}$.

$\operatorname{MS}(\mathbf{E S I}+): m / z(\%)=601(8)\left[\mathrm{M}-\mathrm{BF}_{4}\right]^{+}, 409(100)\left[\mathrm{M}-2 \mathrm{BF}_{4}-\mathrm{SA}\right]^{+}, 305(56)$ $\left[\mathrm{M}-2 \mathrm{BF}_{4}-2 \mathrm{SA}-\mathrm{H}\right]^{+}, 105(85)[\mathrm{SA}]^{+}$.

MS(ESI-): $m / z(\%)=87(100)\left[\mathrm{BF}_{4}\right]^{-}$.

IR (KBr): $\tilde{\nu}\left(\mathrm{cm}^{-1}\right)=3440(\mathrm{br}, \mathrm{m}), 3129(\mathrm{w}), 3079(\mathrm{w}), 1628(\mathrm{w}), 1557(\mathrm{~m}), 1495$ (w), $1452(\mathrm{~m}), 1385(\mathrm{w}), 1300(\mathrm{w}), 1261$ (w), $1199(\mathrm{w}), 1152$ (s), 1081 (s), 1035 (s), $819(\mathrm{w}), 758(\mathrm{~m}), 706(\mathrm{~m}), 650(\mathrm{w}), 621(\mathrm{w})$.

EA(\%): $\mathrm{C}_{33} \mathrm{H}_{34} \mathrm{~B}_{2} \mathrm{~F}_{8} \mathrm{~N}_{6}$ (688.27) berechnet: C 57.59, H 4.98, N 12.21;

gefunden : $\quad$ C 57.44, H 4.98, N 12.30.

Schmelzpunkt: $261^{\circ} \mathrm{C}$. 


\subsubsection{Synthese von Silber-NHC-Komplexen}
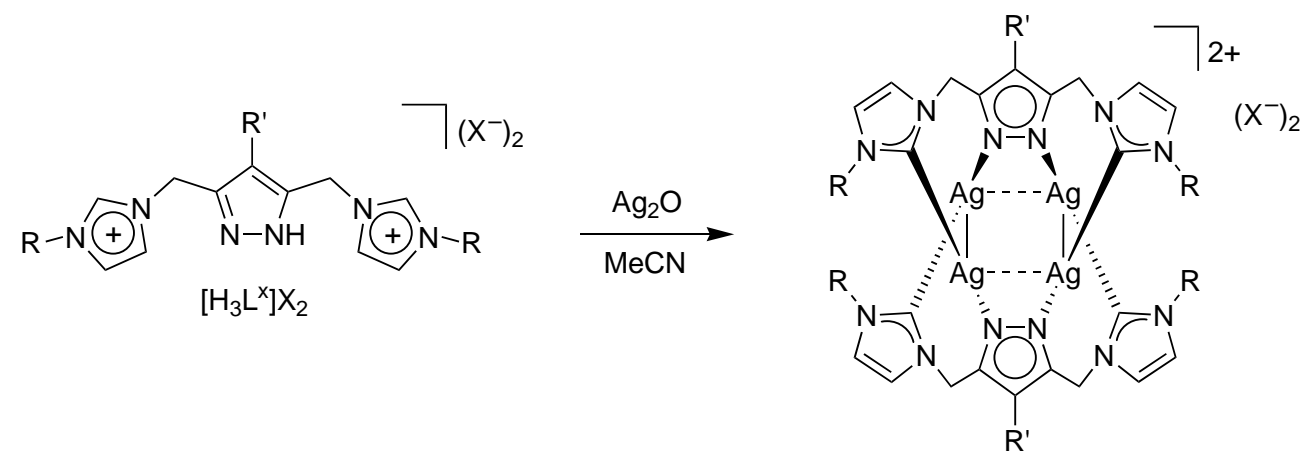

$\left[\left(\mathrm{L}^{\mathrm{X}}\right)_{2} \mathrm{Ag}_{4}\right] \mathrm{X}_{2}$

Basierend auf [35]

Der jeweilige Ligandvorläufer (1.0 Äq) wird in Acetonitril gelöst. Silber(I)oxid (2.2 ̈̈q) wird zugesetzt und die Reaktionsmischung bei RT unter Lichtausschluss für 48 h gerührt. Die Suspension wird mit Aktivkohle versetzt, einige Minuten gerührt und schließlich über Celite 545 filtriert. Aus dem Filtrat wird nach dem Entfernen des Lösungsmittels das Produkt als hellbrauner Feststoff isoliert.

\section{$\left[\left(\mathrm{L}^{1}\right)_{2} \mathrm{Ag}_{4}\right]\left(\mathrm{BF}_{4}\right)_{2}$}

Ausbeute: $1.00 \mathrm{~g}(2.23 \mathrm{mmol})\left[\mathrm{H}_{3} \mathrm{~L}^{1}\right]\left(\mathrm{BF}_{4}\right)_{2} ; 0.44 \mathrm{~g}(0.34 \mathrm{mmol})\left[\left(\mathrm{L}^{1}\right)_{2} \mathrm{Ag}_{4}\right]\left(\mathrm{BF}_{4}\right)_{2}$; $15 \%$. Farblose Kristalle, welche zur Röntgenkristallstrukturanalyse geeignet waren, konnten durch Diethylether-Diffusion in eine MeCN/MeOH-Mischung/Lösung (5:1) von $\left[\left(\mathrm{L}^{1}\right)_{2} \mathrm{Ag}_{4}\right]\left(\mathrm{BF}_{4}\right)_{2}$ bei RT erhalten werden.

${ }^{1} \mathbf{H}-\mathbf{N M R}\left(500 \mathrm{MHz}, \mathrm{CD}_{3} \mathrm{CN}\right): \delta=1.62(\mathrm{~s}, 18 \mathrm{H}, t \mathrm{Bu}), 5.19(\mathrm{~d}, J=15.1 \mathrm{~Hz}, 2 \mathrm{H}$, $\left.\mathrm{CH}_{2}\right), 5.32\left(\mathrm{~d}, J=15.1 \mathrm{~Hz}, 2 \mathrm{H}, \mathrm{CH}_{2}\right), 6.18\left(\mathrm{~s}, 1 \mathrm{H}, \mathrm{CH}^{P z}\right), 7.32(\mathrm{~d}, J=1.9 \mathrm{~Hz}, 2 \mathrm{H}$, $\left.\mathrm{CH}^{I m}\right), 7.33\left(\mathrm{~d}, J=1.9 \mathrm{~Hz}, 2 \mathrm{H}, \mathrm{CH}^{I m}\right) \mathrm{ppm}$.

${ }^{1}$ H-NMR $\left(500 \mathrm{MHz}, \mathrm{DMSO}-d_{6}\right): \delta=1.59(\mathrm{~s}, 18 \mathrm{H}, t \mathrm{Bu}), 5.31(\mathrm{~d}, J=14.8 \mathrm{~Hz}, 2 \mathrm{H}$, $\left.\mathrm{CH}_{2}\right), 5.45$ (d, J=14.8 Hz, 2H, $\left.\mathrm{CH}_{2}\right), 6.27$ (s, $\left.1 \mathrm{H}, \mathrm{CH}^{P z}\right), 7.61$ (d, J=1.8 Hz, $2 \mathrm{H}$, $\left.\mathrm{CH}^{I m}\right), 7.66\left(\mathrm{~d}, J=1.8 \mathrm{~Hz}, 2 \mathrm{H}, \mathrm{CH}^{I m}\right) \mathrm{ppm}$.

${ }^{13}$ C-NMR $\left(125 \mathrm{MHz}, \mathrm{CD}_{3} \mathrm{CN}\right): \delta=32.0(\mathrm{tBu}), 50.5\left(\mathrm{CH}_{2}\right), 58.8\left(\mathrm{C}^{t B u}\right), 103.7$ $\left(\mathrm{CH}^{P z 4}\right), 119.4,123.3\left(2 \times \mathrm{CH}^{I m}\right), 154.1\left(\mathrm{C}^{P z 3,5}\right), 175.5$ (br, $\left.\mathrm{Ag}^{-\mathrm{C}^{I m 2}}\right) \mathrm{ppm}$. 
${ }^{13}$ C-NMR (125MHz, DMSO- $\left.d_{6}\right): \delta=31.2(t \mathrm{Bu}), 48.8\left(\mathrm{CH}_{2}\right), 57.5\left(\mathrm{C}^{t B u}\right), 103.1$ $\left(\mathrm{CH}^{P z 4}\right), 118.9,122.8\left(2 \times \mathrm{CH}^{I m}\right), 152.8\left(\mathrm{C}^{P z 3,5}\right), 173.5$ (br d, J=250 Hz, Ag$\left.\mathrm{C}^{\operatorname{Im} 2}\right) \mathrm{ppm}$.

${ }^{1} 5 \mathrm{~N}-\mathrm{NMR}\left(40.56 \mathrm{MHz}, \mathrm{CD}_{3} \mathrm{CN}\right): \delta=-184.2,-161.7\left(2 \times \mathrm{N}^{I m}\right),-113.4\left(\mathrm{br}, \mathrm{N}^{P z}\right)$.

$\operatorname{MS}(\mathbf{E S I}+): m / z(\%)=1197(3.3)\left[\mathrm{M}-B F_{4}\right]^{+}, 1109(0.5)\left[\mathrm{M}-2 \mathrm{BF}_{4}-\mathrm{H}\right]^{+}, 555(100)$, $\left[\mathrm{Ag}_{2} \mathrm{~L}^{1}\right]^{+}$.

MS(ESI-): $m / z(\%)=87(100)\left[\mathrm{BF}_{4}\right]^{-}$.

UV/Vis: $\lambda=249,226,203 \mathrm{~nm}$.

$\mathbf{E A}(\%): \mathrm{C}_{38} \mathrm{H}_{54} \mathrm{Ag}_{4} \mathrm{~B}_{2} \mathrm{~F}_{8} \mathrm{~N}_{12}$ (1284.00) berechnet: $\quad$ C 35.54, H 4.24, N 13.09; gefunden : $\quad$ C $35.28, \mathrm{H} 4.53, \mathrm{~N} 13.08$.

Schmelzpunkt: $262^{\circ} \mathrm{C}$.

$\left[\left(\mathrm{L}^{3}\right)_{2} \mathrm{Ag}_{4}\right]\left(\mathrm{BF}_{4}\right)_{2}$

Ausbeute: $0.75 \mathrm{~g}(1.12 \mathrm{mmol})\left[\mathrm{H}_{3} \mathrm{~L}^{3}\right]\left(\mathrm{BF}_{4}\right)_{2} ; 0.99 \mathrm{~g}(0.63 \mathrm{mmol})\left[\left(\mathrm{L}^{3}\right)_{2} \mathrm{Ag}_{4}\right]\left(\mathrm{BF}_{4}\right)_{2}$; $56 \%$. Durch langsame Diffusion von Diethylether in eine MeCN-Lösung von $\left[\left(\mathrm{L}^{3}\right)_{2} \mathrm{Ag}_{4}\right]\left(\mathrm{BF}_{4}\right)_{2}$ bei RT konnten farblose Kristalle erhalten werden.

${ }^{1} \mathrm{H}-\mathrm{NMR}\left(500 \mathrm{MHz}, \mathrm{CD}_{3} \mathrm{CN}\right): \delta=1.66\left(\mathrm{~d}, J=12.6 \mathrm{~Hz}, 6 \mathrm{H}, \mathrm{CH}_{2}^{A d}\right), 1.74$ (d, $\left.J=12.6 \mathrm{~Hz}, 6 \mathrm{H}, \mathrm{CH}_{2}^{A d}\right), 2.14\left(\mathrm{~m}, 6 \mathrm{H}, \mathrm{CH}^{A d}\right), 2.23\left(\mathrm{~m}, 12 \mathrm{H}, \mathrm{CH}_{2}^{A d}\right), 5.18$ (d, $\left.J=15.1 \mathrm{~Hz}, 2 \mathrm{H}, \mathrm{CH}_{2}\right), 5.32\left(\mathrm{~d}, J=15.1 \mathrm{~Hz}, 2 \mathrm{H}, \mathrm{CH}_{2}\right), 6.18\left(\mathrm{~s}, 1 \mathrm{H}, \mathrm{CH}^{P z}\right), 7.32$ $\left(\mathrm{d}, J=1.8 \mathrm{~Hz}, 2 \mathrm{H}, \mathrm{CH}^{I m}\right), 7.34\left(\mathrm{~d}, J=1.8 \mathrm{~Hz}, 2 \mathrm{H}, \mathrm{CH}^{I m}\right) \mathrm{ppm}$.

${ }^{13} \mathbf{C}-\mathrm{NMR}\left(125 \mathrm{MHz}, \mathrm{CD}_{3} \mathrm{CN}\right): \delta=30.9\left(\mathrm{CH}^{A d}\right), 36.3\left(\mathrm{CH}-\mathrm{CH}_{2}-\mathrm{CH}^{A d}\right), 45.5(\mathrm{C}-$ $\left.\mathrm{CH}_{2}^{A d}\right), 50.7\left(\mathrm{CH}_{2}\right), 59.1\left(\mathrm{C}^{A d}\right), 103.8\left(\mathrm{CH}^{P z 4}\right), 118.5,123.3\left(2 \times \mathrm{CH}^{I m}\right), 154.2$ $\left(\mathrm{C}^{P z 3,5}\right) \mathrm{ppm}$.

MS(ESI+): $m / z(\%)=1509(0.9)\left[\mathrm{M}-B F_{4}\right]^{+}, 711(100)\left[\mathrm{M}-2 \mathrm{BF}_{4}\right]^{2+}, 363(46)$ $\left[\mathrm{L}^{3}-\mathrm{Ad}\right]^{+}, 135(38)[\mathrm{Ad}]^{+}$.

MS(ESI-): $m / z(\%)=87(100)\left[\mathrm{BF}_{4}\right]^{-}$. 


\section{$\left[\left(\mathrm{L}^{4}\right)_{2} \mathrm{Ag}_{4}\right]\left(\mathrm{PF}_{6}\right)_{2}$}

Ausbeute: $0.58 \mathrm{~g}(0.67 \mathrm{mmol})\left[\mathrm{H}_{3} \mathrm{~L}^{4}\right]\left(\mathrm{PF}_{6}\right)_{2} ; 0.71 \mathrm{~g}(0.38 \mathrm{mmol})\left[\left(\mathrm{L}^{4}\right)_{2} \mathrm{Ag}_{4}\right]\left(\mathrm{PF}_{6}\right)_{2}$; $57 \%$. Durch langsame Diffusion von Diethylether in eine MeCN-Lösung von $\left[\left(\mathrm{L}^{4}\right)_{2} \mathrm{Ag}_{4}\right]\left(\mathrm{PF}_{6}\right)_{2}$ bei RT konnten farblose Kristalle erhalten werden.

${ }^{1} \mathbf{H}-\mathbf{N M R}\left(500 \mathrm{MHz}, \mathrm{CD}_{3} \mathrm{CN}\right): \delta=1.71\left(\mathrm{~d}, J=12.5 \mathrm{~Hz}, 6 \mathrm{H}, \mathrm{CH}_{2}^{A d}\right), 1.78$ (d, $\left.J=12.5 \mathrm{~Hz}, 6 \mathrm{H}, \mathrm{CH}_{2}^{A d}\right), 2.20\left(\mathrm{~m}, 6 \mathrm{H}, \mathrm{CH}^{A d}\right), 2.26\left(\mathrm{~m}, 12 \mathrm{H}, \mathrm{CH}_{2}^{A d}\right), 5.18$ (d, $\left.J=15.4 \mathrm{~Hz}, 2 \mathrm{H}, \mathrm{CH}_{2}\right), 5.25\left(\mathrm{~d}, J=15.4 \mathrm{~Hz}, 2 \mathrm{H}, \mathrm{CH}_{2}\right), 6.37\left(\mathrm{~m}, 2 \mathrm{H}, \mathrm{CH}^{o-P h}\right), 6.66$ $\left(\mathrm{d}, J=1.9 \mathrm{~Hz}, 2 \mathrm{H}, \mathrm{CH}^{I m}\right), 7.05\left(\mathrm{~d}, J=1.9 \mathrm{~Hz}, 2 \mathrm{H}, \mathrm{CH}^{I m}\right), 7.11\left(\mathrm{~m}, 2 \mathrm{H}, \mathrm{CH}^{m-P h}\right)$, $7.17\left(\mathrm{~m}, 1 \mathrm{H}, \mathrm{CH}^{p-P h}\right) \mathrm{ppm}$.

${ }^{13} \mathbf{C}-\mathrm{NMR}\left(125 \mathrm{MHz}, \mathrm{CD}_{3} \mathrm{CN}\right): \delta=30.9\left(\mathrm{CH}^{A d}\right), 36.3\left(\mathrm{CH}-\mathrm{CH}_{2}-\mathrm{CH}^{A d}\right), 45.5(\mathrm{C}-$ $\left.\mathrm{CH}_{2}^{A d}\right), 50.2\left(\mathrm{CH}_{2}\right), 60.0\left(\mathrm{C}^{A d}\right), 118.1\left(\mathrm{CH}^{I m}\right), 121.3\left(\mathrm{C}^{P z 4}\right), 123.3\left(\mathrm{CH}^{I m}\right), 127.9$ $\left(\mathrm{CH}^{p-P h}\right), 129.2\left(\mathrm{CH}^{m-P h}\right), 130.1\left(\mathrm{CH}^{o-P h}\right), 132.4\left(\mathrm{C}^{i-P h}\right), 151.4\left(\mathrm{C}^{P z 3,5}\right) \mathrm{ppm}$.

MS(ESI+) $: m / z(\%)=1719(1.4)\left[{\mathrm{M}-\mathrm{PF}_{6}}^{+}, 787(100)\left[\mathrm{M}-2 \mathrm{PF}_{6}\right]^{2+}, 439(16)\right.$ $\left[\mathrm{L}^{4}-\mathrm{Ad}\right]^{+}, 135(33)[\mathrm{Ad}]^{+}$.

MS(ESI-): $m / z(\%)=145(100)\left[\mathrm{PF}_{6}\right]^{-}$.

IR (KBr): $\tilde{\nu}\left(\mathrm{cm}^{-1}\right)=3466(\mathrm{~m}), 3399(\mathrm{~m}), 3189(\mathrm{w}), 3156(\mathrm{w}), 2908(\mathrm{~m}), 2853$ (m), $1666(\mathrm{~m}), 1606$ (w), 1555 (w), 1448 (m), 1403 (m), 1358 (w), 1300 (w), 1256 (w), $1223(\mathrm{w}), 1173(\mathrm{w}), 1153(\mathrm{w}), 1105(\mathrm{w}), 1074(\mathrm{w}), 1043(\mathrm{w}), 1018(\mathrm{w}), 961(\mathrm{w})$, $842(\mathrm{~s}), 744(\mathrm{~m}), 700(\mathrm{~m}), 669(\mathrm{w}), 557(\mathrm{~m}), 486(\mathrm{w}), 454(\mathrm{w})$.

$\mathbf{E A}(\%): \mathrm{C}_{74} \mathrm{H}_{86} \mathrm{Ag}_{4} \mathrm{~F}_{12} \mathrm{~N}_{12} \mathrm{P}_{2}$ (1864.96) berechnet: $\quad$ C 47.66, H 4.65, N 9.01; gefunden : $\quad$ C $47.52, \mathrm{H} 4.71, \mathrm{~N} 8.98$.

$\left[\left(\mathrm{L}^{6}\right)_{2} \mathrm{Ag}_{4}\right]\left(\mathrm{BF}_{4}\right)_{2}$

Ausbeute: $1.02 \mathrm{~g}(1.27 \mathrm{mmol})\left[\mathrm{H}_{3} \mathrm{~L}^{6}\right]\left(\mathrm{BF}_{4}\right)_{2} ; 1.37 \mathrm{~g}(0.76 \mathrm{mmol}) ; 60 \%$. Durch langsame Diffusion von Diethylether in eine Aceton-Lösung von $\left[\left(\mathrm{L}^{6}\right)_{2} \mathrm{Ag}_{4}\right]\left(\mathrm{BF}_{4}\right)_{2}$ bei RT konnten farblose Kristalle erhalten werden.

${ }^{1} \mathbf{H}-\mathbf{N M R}\left(500 \mathrm{MHz}, \mathrm{CD}_{3} \mathrm{CN}\right): \delta=1.79\left(\mathrm{~s}, 6 \mathrm{H}, \mathrm{CH}_{3}^{M e s 2,6}\right), 1.98\left(\mathrm{~s}, 6 \mathrm{H}, \mathrm{CH}_{3}^{M e s 2,6}\right)$, $2.32\left(\mathrm{~s}, 6 \mathrm{H}, \mathrm{CH}_{3}^{M e s 4}\right), 5.03\left(\mathrm{~d}, J=15.2 \mathrm{~Hz}, 2 \mathrm{H}, \mathrm{CH}_{2}\right), 5.25\left(\mathrm{~d}, J=15.2 \mathrm{~Hz}, 2 \mathrm{H}, \mathrm{CH}_{2}\right)$, $6.78\left(\mathrm{~m}, 2 \mathrm{H}, \mathrm{CH}^{o-P h}\right), 7.01\left(\mathrm{~m}, 4 \mathrm{H}, \mathrm{CH}^{M e s 3,5}\right), 7.04\left(\mathrm{~m}, 2 \mathrm{H}, \mathrm{CH}^{I m}\right), 7.15(\mathrm{~s}, 2 \mathrm{H}$, $\left.\mathrm{CH}^{I m}\right), 7.25\left(\mathrm{~m}, 3 \mathrm{H}, \mathrm{CH}^{m, p-P h}\right) \mathrm{ppm}$.

${ }^{13} \mathbf{C}-\mathrm{NMR}\left(125 \mathrm{MHz}, \mathrm{CD}_{3} \mathrm{CN}\right): \delta=18.1,18.6\left(\mathrm{CH}_{3}^{M e s 2,6}\right), 21.1\left(\mathrm{CH}_{3}^{M e s 4}\right), 49.3$ $\left(\mathrm{CH}_{2}\right), 121.3\left(\mathrm{C}^{P z 4}\right), 123.2,123.3,124.8,124.9\left(\mathrm{CH}^{I m}\right), 128.3\left(\mathrm{CH}^{p-P h}\right), 129.5$, 
$129.9\left(\mathrm{CH}^{o, m-P h}\right), 130.0,130.3\left(\mathrm{CH}^{M e s 3,5}\right), 132.6\left(\mathrm{C}^{i-P h}\right), 135.7,135.8\left(\mathrm{CH}^{M e s 2,6}\right)$, $137.2\left(\mathrm{C}^{\text {Mes1 }}\right), 140.5\left(\mathrm{C}^{M e s 4}\right), 149.2\left(\mathrm{C}^{P z 3,5}\right), 178.5$ (br d, J=250 Hz, Ag- $\left.\mathrm{C}^{I m 2}\right) \mathrm{ppm}$.

MS(ESI+): $m / z(\%)=650(100)\left[\mathrm{L}^{6} \mathrm{Ag}\right]^{+}, 462(5)\left[\mathrm{L}^{6} \mathrm{Ag}-\mathrm{SA}\right]^{+}, 187(3)[\mathrm{SA}+\mathrm{H}]^{+}$.

MS(ESI-): $m / z(\%)=87(100)\left[\mathrm{BF}_{4}\right]^{-}$.

Schmelzpunkt: $141^{\circ} \mathrm{C}$ (Zersetzung).

$$
\left[\left(\mathrm{L}^{10}\right)_{2} \mathrm{Ag}_{4}\right]\left(\mathrm{BF}_{4}\right)_{2}
$$

Ausbeute: $0.9 \mathrm{~g}(1.47 \mathrm{mmol})\left[\mathrm{H}_{3} \mathrm{~L}^{10}\right]\left(\mathrm{BF}_{4}\right)_{2} ; 1.31 \mathrm{~g}(0.89 \mathrm{mmol})\left[\left(\mathrm{L}^{10}\right)_{2} \mathrm{Ag}_{4}\right]\left(\mathrm{BF}_{4}\right)_{2}$; $60 \%$. Durch langsame Diffusion von Diethylether in eine MeCN-Lösung des Rohproduktes bei RT konnten farblose Kristalle erhalten werden.

${ }^{1} \mathbf{H}-\mathbf{N M R}\left(500 \mathrm{MHz}, \mathrm{CD}_{3} \mathrm{CN}\right): \delta=1.72\left(\mathrm{~d}, J=7.1 \mathrm{~Hz}, 3 \mathrm{H}, \mathrm{CH}_{3}\right), 1.82(\mathrm{~d}, J=7.0 \mathrm{~Hz}$, $\left.3 \mathrm{H}, \mathrm{CH}_{3}\right), 4.50$ (d, J=14.8 Hz, $\left.1 \mathrm{H}, \mathrm{CH}_{2}\right), 4.90$ (d, J=14.8 Hz, $1 \mathrm{H}, \mathrm{CH}_{2}$ ), 5.13 (d, $\left.J=14.8 \mathrm{~Hz}, 1 \mathrm{H}, \mathrm{CH}_{2}\right), 5.23\left(\mathrm{~d}, J=15.0 \mathrm{~Hz}, 1 \mathrm{H}, \mathrm{CH}_{2}\right), 5.43(\mathrm{q}, J=5.4 \mathrm{~Hz}, 1 \mathrm{H}, \mathrm{CH})$, $5.71(\mathrm{q}, J=5.7 \mathrm{~Hz}, 1 \mathrm{H}, \mathrm{CH}), 6.22\left(\mathrm{~s}, 1 \mathrm{H}, \mathrm{CH}^{P z}\right), 7.26\left(\mathrm{~m}, 13 \mathrm{H}, \mathrm{CH}^{P h}, \mathrm{CH}^{I m}\right), 7.28$ (d, $\left.J=2.0 \mathrm{~Hz}, 1 \mathrm{H}, \mathrm{CH}^{I m}\right) \mathrm{ppm}$.

${ }^{13}$ C-NMR (125MHz, $\left.\mathrm{CD}_{3} \mathrm{CN}\right): \delta=21.7,21.9\left(2 \times \mathrm{CH}_{3}\right), 49.5,49.8\left(2 \times \mathrm{CH}_{2}\right)$, 61.6, $62.2(2 \times \mathrm{CH}), 103.8\left(\mathrm{CH}^{P z}\right), 119.1,120.0\left(\mathrm{CH}^{I m}\right), 124.6,124.7\left(\mathrm{CH}^{I m}\right), 127.6$, $127.8\left(\mathrm{CH}^{o-P h}\right), 129.3\left(\mathrm{CH}^{p-P h}\right), 129.8,129.9\left(\mathrm{CH}^{m-P h}\right), 141.9,143.0,152.3,153.0$ $\left(\mathrm{C}^{P z 3,5}\right), 176.4$ (br d, $\left.J=259.0 \mathrm{~Hz}, \mathrm{Ag}_{-} \mathrm{C}^{I m 2}\right), 176.7$ (dd, $J=251.1 \mathrm{~Hz}, J=17.9 \mathrm{~Hz}$, $\left.\mathrm{Ag}-\mathrm{C}^{\operatorname{Im} 2}\right) \mathrm{ppm}$.

$\operatorname{MS}(\mathbf{E S I}+): m / z(\%)=1389(4)\left[\mathrm{M}-B F_{4}\right]^{+}, 651(100)\left[\mathrm{M}-2 \mathrm{BF}_{4}\right]^{2+}, 107(85)[\mathrm{Ag}]^{+}$.

MS(ESI-): $m / z(\%)=87(100)\left[\mathrm{BF}_{4}\right]^{-}$.

\section{$\left[\left(\mathrm{L}^{10}\right)_{2} \mathrm{Ag}_{6}(\text { Aceton })_{2}\right]\left(\mathrm{BF}_{4}\right)_{4}$}

Durch langsame Diffusion von Diethylether in eine Aceton-Lösung des Rohproduktes bei RT konnten farblose Kristalle erhalten werden.

${ }^{1}$ H-NMR (400MHz, Aceton- $\left.d_{6}\right): \delta=1.83$ (d, $\left.J=7.1 \mathrm{~Hz}, 3 \mathrm{H}, \mathrm{CH}_{3}\right), 1.88$ (d, $\left.J=7.0 \mathrm{~Hz}, 3 \mathrm{H}, \mathrm{CH}_{3}\right), 4.82\left(\mathrm{~d}, J=14.8 \mathrm{~Hz}, 1 \mathrm{H}, \mathrm{CH}_{2}\right), 5.17\left(\mathrm{~d}, J=14.8 \mathrm{~Hz}, 1 \mathrm{H}, \mathrm{CH}_{2}\right)$, $5.46\left(\mathrm{~d}, J=14.8 \mathrm{~Hz}, 1 \mathrm{H}, \mathrm{CH}_{2}\right), 5.52\left(\mathrm{~d}, J=15.0 \mathrm{~Hz}, 1 \mathrm{H}, \mathrm{CH}_{2}\right), 5.07$ (q, J=5.4 Hz, $1 \mathrm{H}, \mathrm{CH}), 5.85(\mathrm{q}, J=5.7 \mathrm{~Hz}, 1 \mathrm{H}, \mathrm{CH}), 6.40\left(\mathrm{~s}, 1 \mathrm{H}, \mathrm{CH}^{P z}\right), 7.28\left(\mathrm{~m}, 5 \mathrm{H}, \mathrm{CH}^{P h}\right)$, $7.34\left(\mathrm{~m}, 5 \mathrm{H}, \mathrm{CH}^{P h}\right), 7.57,7.59,7.62,7.66$ (alle s, $\left.4 \mathrm{H}, \mathrm{CH}^{I m}\right) \mathrm{ppm}$. 
${ }^{13}$ C-NMR $\left(125 \mathrm{MHz}\right.$, Aceton- $\left.d_{6}\right): \delta=21.2,21.4\left(2 \times \mathrm{CH}_{3}\right), 48.9,49.1\left(2 \mathrm{x} \mathrm{CH}_{2}\right)$, 61.2, $61.9(2 \times \mathrm{CH}), 103.2\left(\mathrm{CH}^{P z}\right), 118.7,119.9\left(\mathrm{CH}^{I m}\right), 124.5\left(\mathrm{br}, \mathrm{CH}^{I m}\right), 127.2$, $127.3\left(\mathrm{CH}^{o-P h}\right), 128.7,128.8\left(\mathrm{CH}^{p-P h}\right), 129.3,129.4\left(\mathrm{CH}^{m-P h}\right), 141.4,141.9,152.3$, $152.6\left(\mathrm{C}^{P z 3,5}\right) \mathrm{ppm}$.

MS(ESI+): $m / z(\%)$ in Aceton $=1387(4)\left[\mathrm{M}-B F_{4}\right]^{+}, 651(100)\left[\mathrm{M}-2 \mathrm{BF}_{4}\right]^{2+}, 109$ (55) $[\mathrm{Ag}]^{+}$.

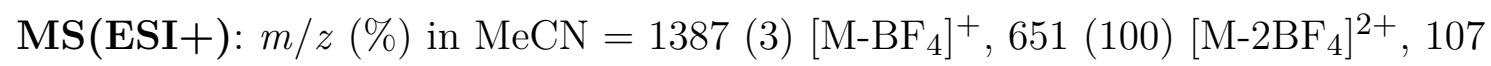
(20) $[\mathrm{Ag}]^{+}$.

MS(ESI-): $m / z(\%)=87(100)\left[\mathrm{BF}_{4}\right]^{-}$.

Schmelzpunkt: $281^{\circ} \mathrm{C}$ (Zersetzung).

$\left[\left(\mathrm{L}^{11}\right)_{2} \mathrm{Ag}_{4}\right]\left(\mathrm{PF}_{6}\right)_{2}$

Ausbeute: $1.00 \mathrm{~g}(1.24 \mathrm{mmol})\left[\mathrm{H}_{3} \mathrm{~L}^{11}\right]\left(\mathrm{PF}_{6}\right)_{2} ; 1.21 \mathrm{~g}(0.69 \mathrm{mmol})\left[\left(\mathrm{L}^{11}\right)_{2} \mathrm{Ag}_{4}\right]\left(\mathrm{PF}_{6}\right)_{2}$; $56 \%$.

${ }^{1} \mathbf{H}-\mathbf{N M R}\left(500 \mathrm{MHz}, \mathrm{CD}_{3} \mathrm{CN}\right): \delta=1.76\left(\mathrm{~d}, J=7.1 \mathrm{~Hz}, 3 \mathrm{H}, \mathrm{CH}_{3}\right), 1.81(\mathrm{~d}, J=7.1 \mathrm{~Hz}$, $\left.3 \mathrm{H}, \mathrm{CH}_{3}\right), 4.61\left(\left(\mathrm{~d}, J=15.4 \mathrm{~Hz}, 1 \mathrm{H}, \mathrm{CH}_{2}\right), 4.95\right.$ (d, J=15.4 Hz, $\left.1 \mathrm{H}, \mathrm{CH}_{2}\right), 5.03$ (d, $\left.J=15.4 \mathrm{~Hz}, 1 \mathrm{H}, \mathrm{CH}_{2}\right), 5.20\left(\mathrm{~d}, J=15.4 \mathrm{~Hz}, 1 \mathrm{H}, \mathrm{CH}_{2}\right), 5.51$ (q, J=7.0 Hz, $1 \mathrm{H}, \mathrm{CH}$ ), $5.75(\mathrm{q}, J=7.0 \mathrm{~Hz}, 1 \mathrm{H}, \mathrm{CH}), 6.49\left(\mathrm{~m}, 2 \mathrm{H}, \mathrm{CH}^{P h}\right), 6.79(\mathrm{dd}, J=1.8 \mathrm{~Hz}, J=25.1 \mathrm{~Hz}$, $\left.2 \mathrm{H}, \mathrm{CH}^{I m}\right), 6.93\left(\mathrm{~d}, J=1.8 \mathrm{~Hz}, 1 \mathrm{H}, \mathrm{CH}^{I m}\right), 7.27\left(\mathrm{~m}, 14 \mathrm{H}, \mathrm{CH}^{P h}, \mathrm{CH}^{I m}\right) \mathrm{ppm}$.

${ }^{13}$ C-NMR $\left(125 \mathrm{MHz}, \mathrm{CD}_{3} \mathrm{CN}\right): \delta=21.8\left(\mathrm{CH}_{3}\right), 48.9,49.3\left(2 \times \mathrm{CH}_{2}\right), 61.5,62.2$ (2 x CH), 118.9, $119.4\left(\mathrm{CH}^{I m}\right), 120.9\left(\mathrm{CH}^{P z 4}\right), 124.5,124.7\left(\mathrm{CH}^{I m}\right), 127.6,127.7$ $\left(\mathrm{CH}^{o, m-P h}\right), 128.0\left(\mathrm{CH}^{p-P h}\right), 129.1\left(\mathrm{CH}^{p-P h}\right), 129.3\left(\mathrm{CH}^{m-P h}\right), 129.7\left(\mathrm{CH}^{p-P h}\right)$, $129.9\left(\mathrm{CH}^{o-P h}\right), 130.0,132.6\left(\mathrm{C}^{i-P h}\right), 142.1,142.9,149.6,150.4\left(\mathrm{C}^{P z 3,5}\right) \mathrm{ppm}$.

MS(ESI+): $m / z(\%)=1599(5)\left[\mathrm{M}-\mathrm{PF}_{6}\right]^{+}, 727(100)\left[\mathrm{M}-2 \mathrm{PF}_{6}\right]^{2+}, 107(100)[\mathrm{Ag}]^{+}$.

MS(ESI-): $m / z(\%)=145(100)\left[\mathrm{PF}_{6}\right]^{-}$.

EA(\%): $\mathrm{C}_{66} \mathrm{H}_{62} \mathrm{Ag}_{4} \mathrm{~F}_{12} \mathrm{~N}_{12} \mathrm{P}_{2}$ (1744.68) berechnet: C 45.44, H 3.58, N 9.63; gefunden : $\quad$ C 45.03, H 6.63, N 9.32. 


\section{Synthese von $\left[\left(\mathrm{H}_{2} \mathrm{~L}^{1}\right)_{4} \mathrm{Ag}_{4}\right]\left(\mathrm{PF}_{6}\right)_{8}$}

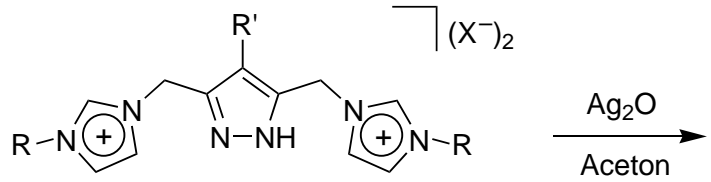

$\left[\mathrm{H}_{3} \mathrm{~L}^{\mathrm{X}}\right] \mathrm{X}_{2}$

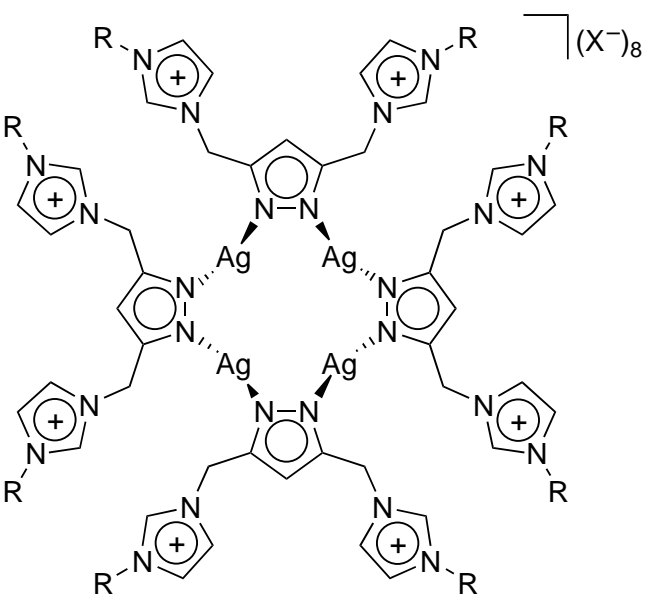

$\left[\left(\mathrm{H}_{2} \mathrm{~L}^{\mathrm{X}}\right)_{4} \mathrm{Ag}_{4}\right] \mathrm{X}_{8}$

Basierend auf [35]

Der jeweilige Ligandvorläufer (1.0 Äq) wird in Aceton gelöst. Silber(I)oxid (2.2 Äq) wird zugesetzt und die Reaktionsmischung bei RT unter Lichtausschluss für $48 \mathrm{~h}$ gerührt. Die Suspension wird mit Aktivkohle versetzt, einige Minuten gerührt und schließlich über Celite 545 filtriert. Aus dem Filtrat wird nach dem Entfernen des Lösungsmittels das Produkt als bräunlicher Feststoff isoliert.

Ausbeute: $\quad 3.00 \mathrm{~g} \quad(4.74 \mathrm{mmol}) \quad\left[\mathrm{H}_{3} \mathrm{~L}^{1}\right]\left(\mathrm{PF}_{6}\right)_{2} ; \quad 1.16 \mathrm{~g} \quad(0.39 \mathrm{mmol})$ $\left[\left(\mathrm{H}_{2} \mathrm{~L}^{1}\right)_{4} \mathrm{Ag}_{4}\right]\left(\mathrm{PF}_{6}\right)_{8} ; 9 \%$. Durch langsame Diffusion von Diethylether in eine Acetonlösung des Rohproduktes bei RT konnten farblose Kristalle erhalten werden.

${ }^{1} \mathrm{H}-\mathrm{NMR}\left(500 \mathrm{MHz}, \mathrm{CD}_{3} \mathrm{CN}\right): \delta=1.59(\mathrm{~s}, 18 \mathrm{H}, t \mathrm{Bu}), 5.39\left(\mathrm{~s}, 4 \mathrm{H}, \mathrm{CH}_{2}\right), 6.44(\mathrm{~s}$, $\left.1 \mathrm{H}, \mathrm{CH}^{P z}\right), 7.35\left(\mathrm{t}, J=1.6 \mathrm{~Hz}, 2 \mathrm{H}, \mathrm{CH}^{I m}\right), 7.56\left(\mathrm{t}, J=1.8 \mathrm{~Hz}, 2 \mathrm{H}, \mathrm{CH}^{I m}\right), 8.55$ (t, $\left.J=1.6 \mathrm{~Hz}, 2 \mathrm{H}, \mathrm{CH}^{\operatorname{Im} 2}\right) \mathrm{ppm}$.

${ }^{13} \mathbf{C}-\mathrm{NMR}\left(125 \mathrm{MHz}, \mathrm{CD}_{3} \mathrm{CN}\right): \delta=29.6(t \mathrm{Bu}), 48.2\left(\mathrm{br}, \mathrm{CH}_{2}\right), 61.4\left(\mathrm{C}^{t B u}\right), 104.8$ $\left(\mathrm{CH}^{P z}\right), 121.4,123.3\left(2 \times \mathrm{CH}^{I m}\right), 134.7\left(\mathrm{CH}^{I m 2}\right), 148.4$ (br, $\left.\mathrm{C}^{P z 3,5}\right) \mathrm{ppm}$.

${ }^{15} \mathrm{~N}-\mathrm{NMR}\left(40.56 \mathrm{MHz}, \mathrm{CD}_{3} \mathrm{CN}\right): \quad \delta=-200.3,-174.8\left(2 \mathrm{x} \quad \mathrm{N}^{I m}\right),-110.3$ $\left(\mathrm{N}^{P z}\right) \mathrm{ppm}$.

$\operatorname{MS}(\mathbf{E S I}+): m / z(\%)=555(5)\left[\mathrm{Ag}_{2} \mathrm{~L}^{1}\right]^{+}, 341(27)\left[\mathrm{L}^{1}-\mathrm{H}\right]^{+}, 285(100)\left[\mathrm{L}^{1}-\mathrm{H}-t \mathrm{Bu}\right]^{+}$, $229(98)\left[\mathrm{L}^{1}-\mathrm{H}-2 t \mathrm{Bu}\right]^{+}$.

MS(ESI-): $m / z(\%)=145(100)\left[\mathrm{PF}_{6}\right]^{-}$. 
EA(\%): $\mathrm{C}_{76} \mathrm{H}_{116} \mathrm{Ag}_{4} \mathrm{~F}_{48} \mathrm{~N}_{24} \mathrm{P}_{8}, 3$ Aceton (3145.33)

$\begin{array}{ll}\text { berechnet: } & \text { C 32.60, H 4.31, N 10.73; } \\ \text { gefunden : } & \text { C 32.14, H 4.37, N 10.64. }\end{array}$

Schmelzpunkt: $219^{\circ} \mathrm{C}$ (Zersetzung).

\subsubsection{Synthese von Palladium-NHC-Komplexen}

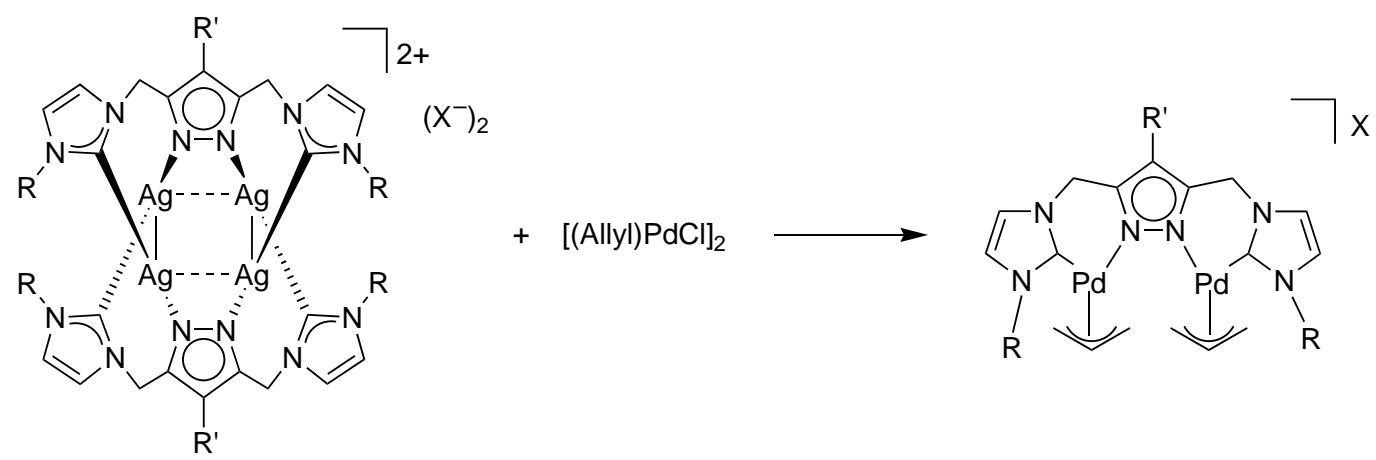

$\left[\left(\mathrm{L}^{\mathrm{X}}\right)_{2} \mathrm{Ag}_{4}\right] \mathrm{X}_{2} ; \mathrm{X}=\mathrm{BF}_{4}, \mathrm{PF}_{6}$

Basierend auf [35]

Unter Lichtausschluss wird zu einer Acetonitrillösung des jeweilligen Silberkomplexes (1.0 ̈̈q) der Palladiumprecursor (Allylpalladium(II)-chlorid Dimer) (2.0Äq) direkt als Feststoff oder als Acetonitrillösung zugesetzt und für 24h bei RT gerührt. Anschließend wird die Reaktionsmischung über Aktivkohle und Celite 545 filtriert. Das erhaltene Filtrat wird unter vermindertem Druck stark eingeengt. Zu der resultierenden konzentrierten Lösung wird unter Rühren Diethylether zugegeben, wobei ein Niederschlag ausfällt, welcher durch Filtration abgetrennt wird und im Vakuum getrocknet. Das Produkt wird als blass gelber Feststoff erhalten.

\section{$\left[\mathrm{L}^{1} \mathrm{Pd}_{2}(\mathrm{Allyl})_{2}\right] \mathrm{BF}_{4}$}

Durch langsame Diffusion von Diethylether in eine Acetonitrillösung des Rohproduktes bei RT konnten farblose Kristalle erhalten werden, welche sich zur röntgenographischen Strukturanalyse eigneten. 
${ }^{1} \mathrm{H}-\mathrm{NMR}\left(500 \mathrm{MHz},-25^{\circ} \mathrm{C}, \mathrm{CD}_{3} \mathrm{CN}\right): \delta=1.62\left(\mathrm{~s}, 18 \mathrm{H}, t \mathrm{Bu}^{A S 1}\right), 1.64(\mathrm{~s}, 18 \mathrm{H}$, $\left.t \mathrm{Bu}^{S 1}\right), 1.71\left(\mathrm{~s}, 18 \mathrm{H}, t \mathrm{Bu}^{S 2}\right), 1.73\left(\mathrm{~s}, 18 \mathrm{H}, t \mathrm{Bu}^{A S 2}\right), 2.61(\mathrm{~d}, J=12.1 \mathrm{~Hz}, 2 \mathrm{H}$,

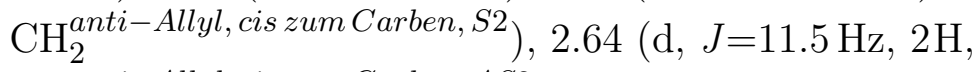

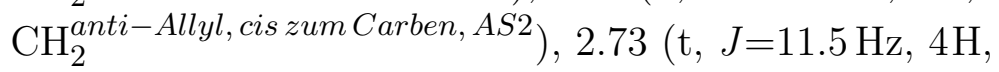
$\left.\mathrm{CH}_{2}^{\text {anti-Allyl, cis zum Carben, } S 1, A S 1}\right), 3.02(\mathrm{~d}, J=13.4 \mathrm{~Hz}, 2 \mathrm{H}$,

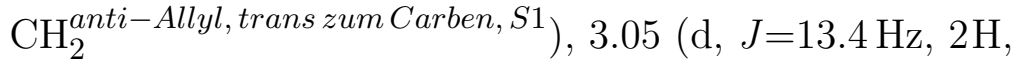
$\mathrm{CH}_{2}^{\text {anti-Allyl,trans zum Carben, } A S 1}$ ), $3.22(\mathrm{~d}, J=13.4 \mathrm{~Hz}, 2 \mathrm{H}$,

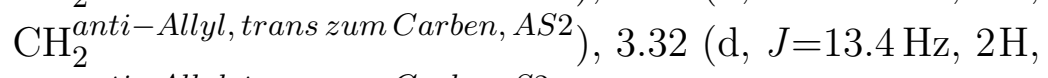

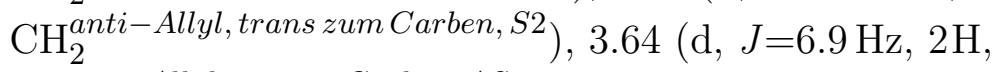
$\mathrm{CH}_{2}^{\text {syn-Allyl, cis zum Carben, } A S 2}$ ), 3.68 (d, $J=6.9 \mathrm{~Hz}, 2 \mathrm{H}$,

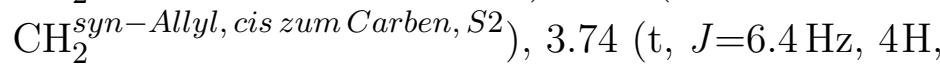

$\mathrm{CH}_{2}^{\text {syn-Allyl, cis zum Carben, } S 1, A S 1}$ ), $3.92(\mathrm{dd}, J=7.4 \mathrm{~Hz}, J=2.0 \mathrm{~Hz}, 2 \mathrm{H}$, $\mathrm{CH}_{2}^{\text {syn-Allyl,trans zum Carben, } A S 2}$ ), 3.97 (dd, $J=7.4 \mathrm{~Hz}, J=2.0 \mathrm{~Hz}, 2 \mathrm{H}$,

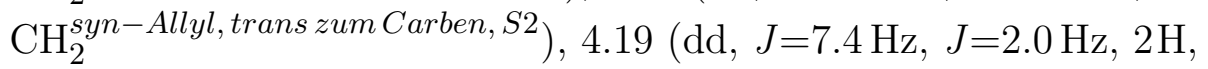
$\mathrm{CH}_{2}^{\text {syn-Allyl,trans zum Carben, } S 1}$ ), $4.26(\mathrm{dd}, J=7.4 \mathrm{~Hz}, J=2.0 \mathrm{~Hz}, 2 \mathrm{H}$, $\mathrm{CH}_{2}^{\text {syn-Allyl,trans zum Carben, } A S 1}$ ), $4.82\left(\mathrm{~d}, J=15.0 \mathrm{~Hz}, 2 \mathrm{H}, \mathrm{CH}_{2}^{A S 1}\right), 4.84(\mathrm{~d}$, $\left.J=15.0 \mathrm{~Hz}, 2 \mathrm{H}, \mathrm{CH}_{2}^{A S 1}\right), 4.93\left(\mathrm{~d}, J=15.0 \mathrm{~Hz}, 4 \mathrm{H}, \mathrm{CH}_{2}^{S 2}\right), 5.05$ (d, J=15.0 Hz, 2H, $\left.\mathrm{CH}_{2}^{S 1}\right), 5.08$ (d, J=15.0 Hz, $\left.2 \mathrm{H}, \mathrm{CH}_{2}^{S 1}\right), 5.18$ (d, J=15.0 Hz, $2 \mathrm{H}, \mathrm{CH}_{2}^{A S 2}$ ), 5.19 (d, $\left.J=15.0 \mathrm{~Hz}, 2 \mathrm{H}, \mathrm{CH}_{2}^{A S 2}\right), 5.26\left(\mathrm{~m}, 4 \mathrm{H}, \mathrm{CH}^{A l l y l}, S 1, S 2\right), 5.63(\mathrm{~m}, 4 \mathrm{H}$,

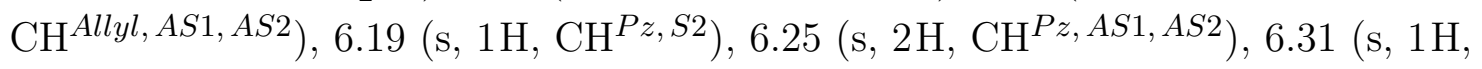
$\left.\mathrm{CH}^{P z, S 1}\right), 7.18\left(\mathrm{~d}, J=1.8 \mathrm{~Hz}, 2 \mathrm{H}, \mathrm{CH}^{I m, A S 1}\right), 7.19\left(\mathrm{~d}, J=1.8 \mathrm{~Hz}, 2 \mathrm{H}, \mathrm{CH}^{I m, A S 1}\right)$, $7.24\left(\mathrm{~d}, J=1.8 \mathrm{~Hz}, 2 \mathrm{H}, \mathrm{CH}^{I m, S 1}\right), 7.27$ (d, J=1.8 Hz, 4H, $\left.\mathrm{CH}^{I m, S 2}\right), 7.28$ (d, $\left.J=1.8 \mathrm{~Hz}, 4 \mathrm{H}, \mathrm{CH}^{I m, A S 2}\right), 7.30\left(\mathrm{~d}, J=1.8 \mathrm{~Hz}, 2 \mathrm{H}, \mathrm{CH}^{I m, S 1}\right) \mathrm{ppm}$.

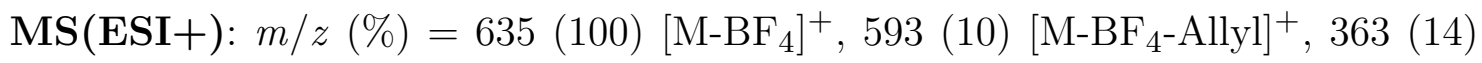
$\left[\mathrm{M}-B F_{4}-2 \mathrm{Allyl}-\mathrm{SA}-t \mathrm{Bu}\right]^{+}$.

MS(ESI-): $m / z(\%)=87(100)\left[\mathrm{BF}_{4}\right]^{-}$.

HR-MS(ESI+): $m / z(\%)$ : für $\mathrm{C}_{25} \mathrm{H}_{37} \mathrm{~N}_{6} \mathrm{Pd}_{2}\left(\left[\mathrm{M}-\mathrm{BF}_{4}\right]^{+}\right)$

berechnet: $\quad 633.1149$;

gefunden : $\quad 633.1148$.

IR (KBr): $\tilde{\nu}\left(\mathrm{cm}^{-1}\right)=3433(\mathrm{br}, \mathrm{s}), 3181(\mathrm{~m}), 3143(\mathrm{~m}), 2976(\mathrm{~m}), 2932(\mathrm{w}), 2881$ (w), $1629(\mathrm{w}), 1561(\mathrm{w}), 1474(\mathrm{w}), 1447(\mathrm{w}), 1410(\mathrm{~m}), 1370(\mathrm{~m}), 1325(\mathrm{w}), 1299$ (w), $1239(\mathrm{~m}), 1209(\mathrm{w}), 11197(\mathrm{w}), 1117(\mathrm{~m}), 1080(\mathrm{~s}), 1034(\mathrm{~s}), 945(\mathrm{w}), 900(\mathrm{w})$, $725(\mathrm{~m}), 679(\mathrm{~m}), 522(\mathrm{w}), 498(\mathrm{w})$.

Schmelzpunkt: $195^{\circ} \mathrm{C}$ (Zersetzung). 


\section{$\left[\mathrm{L}^{6} \mathrm{Pd}_{2}(\mathrm{Allyl})_{2}\right] \mathrm{PF}_{6}$}

Durch langsame Diffusion von Diethylether in eine Acetonitrillösung des Rohproduktes bei RT konnten farblose Kristalle erhalten werden, welche sich zur röntgenographischen Strukturanalyse eigneten.

${ }^{1} \mathbf{H}-\mathbf{N M R}\left(500 \mathrm{MHz}, \mathrm{CD}_{3} \mathrm{CN}\right): \delta=1.80(\mathrm{~d}, J=12.1 \mathrm{~Hz}, 2 \mathrm{H}$, $\left.\mathrm{CH}_{2}^{\text {anti-Allyl, cis zum Carben, } B}\right), 1.90\left(\mathrm{~s}, 6 \mathrm{H}, \mathrm{CH}_{3}^{A r 4, A}\right), 1.93(\mathrm{~d}, J=8.4 \mathrm{~Hz}, 2 \mathrm{H}$, $\mathrm{CH}_{2}^{\text {anti-Allyl, cis zum Carben, } A}$ ), $1.99\left(\mathrm{~s}, 6 \mathrm{H}, \mathrm{CH}_{3}^{A r 4, B}\right), 2.02\left(\mathrm{~s}, 12 \mathrm{H}, \mathrm{CH}_{3}^{A r 2,6, A}\right)$, $2.35\left(\mathrm{~d}, J=4.9 \mathrm{~Hz}, 12 \mathrm{H}, \mathrm{CH}_{3}^{A r 2,6, B}\right), 2.38(\mathrm{~d}, J=7.0 \mathrm{~Hz}, 2 \mathrm{H}$,

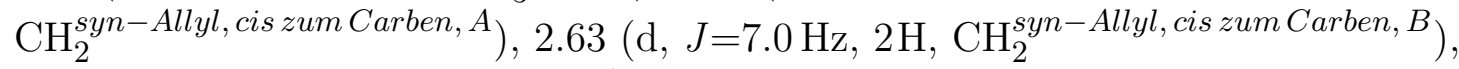
$2.77\left(\mathrm{~d}, J=13.7 \mathrm{~Hz}, 2 \mathrm{H}, \mathrm{CH}_{2}^{\text {anti-Allyl,trans zum Carben, } B}\right), 2.92(\mathrm{~d}, J=13.9 \mathrm{~Hz}, 2 \mathrm{H}$,

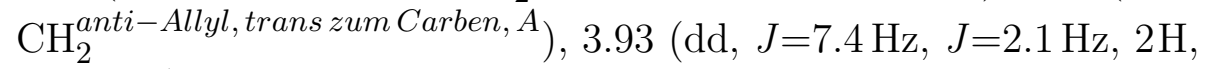
$\left.\mathrm{CH}_{2}^{\text {syn-Allyl,trans zum Carben, } B}\right), 4.05(\mathrm{dd}, J=7.4 \mathrm{~Hz}, J=2.1 \mathrm{~Hz}, 2 \mathrm{H}$, $\left.\mathrm{CH}_{2}^{\text {syn-Allyl,trans zum Carben, } A}\right), 4.90\left(\mathrm{~m}, 4 \mathrm{H}, \mathrm{CH}^{\text {Allyl, } A, B}\right), 5.21(\mathrm{~d}, J=15.6 \mathrm{~Hz}, 2 \mathrm{H}$, $\left.\mathrm{CH}_{2}^{B}\right), 5.30\left(\mathrm{~d}, J=15.6 \mathrm{~Hz}, 2 \mathrm{H}, \mathrm{CH}_{2}^{B}\right), 5.32\left(\mathrm{~s}, 4 \mathrm{H}, \mathrm{CH}_{2}^{A}\right), 6.50\left(\mathrm{~s}, 1 \mathrm{H}, \mathrm{CH}^{P z}, B\right)$, $6.53\left(\mathrm{~s}, 1 \mathrm{H}, \mathrm{CH}^{P z}, A\right), 6.91\left(\mathrm{t}, J=2.0 \mathrm{~Hz}, 4 \mathrm{H}, \mathrm{CH}^{I} m\right), 6.96\left(\mathrm{~m}, 8 \mathrm{H}, \mathrm{CH}^{A r 3,5, A, B}\right)$, $7.43\left(\mathrm{~d}, J=1.8 \mathrm{~Hz}, 2 \mathrm{H}, \mathrm{CH}^{I m}\right), 7.45\left(\mathrm{~d}, J=1.8 \mathrm{~Hz}, 2 \mathrm{H}, \mathrm{CH}^{I m}\right) \mathrm{ppm}$.

${ }^{13} \mathbf{C}-\mathrm{NMR}\left(125 \mathrm{MHz}, \mathrm{CD}_{3} \mathrm{CN}\right): \delta=17.6\left(\mathrm{CH}_{3}^{A r 4, A}\right), 17.9\left(\mathrm{CH}_{3}^{A r 4, B}\right), \quad 18.0$ $\left(\mathrm{CH}_{3}^{A r 2,6, A}\right), 18.1\left(\mathrm{CH}_{3}^{A r 2,6, B}\right), 21.1\left(\mathrm{CH}_{3}^{A r 2,6, A, B}\right), 46.3\left(\mathrm{CH}_{2}^{\text {Allyl }}\right), 47.5\left(\mathrm{CH}_{2}^{\text {Allyl }}\right)$, $48.4\left(\mathrm{CH}_{2}\right), 48.5\left(\mathrm{CH}_{2}\right), 69.0\left(\mathrm{CH}_{2}^{\text {Allyl }}\right), 70.6\left(\mathrm{CH}_{2}^{\text {Allyl }}\right), 102.2\left(\mathrm{CH}^{P z}\right), 102.3\left(\mathrm{CH}^{P z}\right)$, $116.6\left(\mathrm{CH}^{\text {Allyl }}\right), 117.0\left(\mathrm{CH}^{\text {Allyl }}\right), 121.4\left(\mathrm{CH}^{I m}\right), 121.5\left(\mathrm{CH}^{I m}\right), 122.8\left(\mathrm{CH}^{I m}\right)$, $123.0\left(\mathrm{CH}^{I m}\right), 128.8\left(\mathrm{CH}^{A r 3,5, A}\right), 129.0\left(\mathrm{CH}^{A r 3,5, A, B}\right), 129.1\left(\mathrm{CH}^{A r 3,5, B}\right), 135.0$ $\left(\mathrm{CH}^{A r 4, B}\right), 135.2\left(\mathrm{CH}^{A r 4, A}\right), 135.3\left(\mathrm{CH}^{A r 2,6}\right), 136.3\left(\mathrm{CH}^{A r 2,6}\right), 136.4\left(\mathrm{CH}^{A r 2,6}\right)$, $139.4\left(\mathrm{CH}^{A r 1 B}\right), 144.7\left(\mathrm{CH}^{A r 1, A}\right), 176.8\left(\mathrm{C}^{\operatorname{Im} 2, A}\right), 176.9\left(\mathrm{C}^{\operatorname{Im} 2, B}\right) \mathrm{ppm}$.

MS(ESI+): $m / z(\%)=759(100)\left[{\mathrm{M}-\mathrm{PF}_{6}}^{+}\right.$.

MS(ESI-): $m / z(\%)=145(100)\left[\mathrm{PF}_{6}\right]^{-}$.

HR-MS(ESI+): $m / z(\%)$ : für $\mathrm{C}_{35} \mathrm{H}_{41} \mathrm{~N}_{6} \mathrm{Pd}_{2}\left(\left[\mathrm{M}-\mathrm{PF}_{6}\right]^{+}\right)$

$$
\begin{array}{ll}
\text { berechnet: } & 757.1462 ; \\
\text { gefunden : } & 757.1464 .
\end{array}
$$

Schmelzpunkt: $147^{\circ} \mathrm{C}$ (Zersetzung).

$\left[\mathrm{L}^{10} \mathrm{Pd}_{2}(\text { Methallyl })_{2}\right] \mathrm{BF}_{4}$

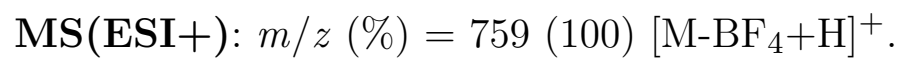

MS(ESI-): $m / z(\%)=87(100)\left[\mathrm{BF}_{4}\right]^{-}$. 
$\left[\mathrm{L}^{11} \mathrm{Pd}_{2}(\text { Allyl })_{2}\right] \mathrm{PF}_{6}$

$\operatorname{MS}(\mathbf{E S I}+): m / z(\%)=807(100)\left[\mathrm{M}-\mathrm{PF}_{6}+\mathrm{H}\right]^{+}$.

MS(ESI-): $m / z(\%)=145(100)\left[\mathrm{PF}_{6}\right]^{-}$.

HR-MS(ESI+): $m / z(\%)$ : für $\mathrm{C}_{39} \mathrm{H}_{41} \mathrm{~N}_{6} \mathrm{Pd}_{2}\left(\left[\mathrm{M}-\mathrm{PF}_{6}\right]^{+}\right)$

$$
\begin{array}{ll}
\text { berechnet: } & 805.1462 ; \\
\text { gefunden : } & 805.1469 .
\end{array}
$$

\subsubsection{Synthese von Nickel-NHC-Komplexen}

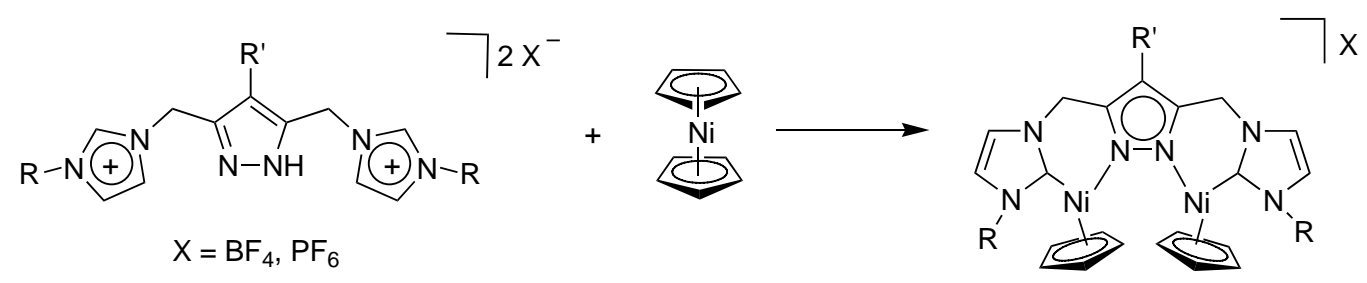

Basierend auf [127]

Unter einer trockenen Stickstoffatmosphäre wird Nickelocen $\left(\mathrm{NiCp}_{2}\right)(2.0 \mathrm{~A} q)$ in trockenem THF gelöst. Der Ligandvorläufer (1.0Äq) wird in THF suspendiert und hinzugegeben. Das Reaktionsgemisch wird für zwei Tage unter Rückfluss erhitzt. Das Lösungsmittel wird im Hochvakuum entfernt, woraufhin das Produkt als rotbrauner Feststoff erhalten wird. Das Rohprodukt wird anschließend mit warmen Toluol $\left(100^{\circ} \mathrm{C}\right)$ extrahiert und nach dem Entfernen des Lösungsmittels wird der rot-braune Feststoff zur Kristallisation angesetzt.

$\left[\mathrm{L}^{5} \mathrm{Ni}_{2} \mathrm{Cp}_{2}\right]\left(\mathrm{BF}_{4}\right)$

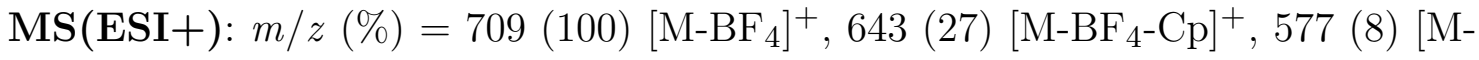
$\left.\mathrm{BF}_{4}-2 \mathrm{Cp}\right]^{+}$.

MS(ESI-): $m / z(\%)=87(100)\left[\mathrm{BF}_{4}\right]^{-}$. 


\subsubsection{Palladium-katalysierte allylische Alkylierung}

Substratsynthese: (rac)-(E)-1,3-Diphenylallylacetat<smiles>OC(/C=C/c1ccccc1)c1ccccc1</smiles><smiles>CCOC(=O)OCC</smiles><smiles>CC(=O)OC(/C=C/c1ccccc1)c1ccccc1</smiles>

Basierend auf [141]

(rac)-(E)-1,3-Diphenylallylalkohol (5.00g, 23.8mmol, 1.0Äq.) und Triethylamin (4.82 g, 6.6ml, 47.6mmol, 2.0 Äq.) werden in absolutiertem Dichlormethan gelöst und auf $0^{\circ} \mathrm{C}$ gekühlt. Nach langsamer Zugabe von Essigsaeureanhydrid $(4.87 \mathrm{~g}, 4.5 \mathrm{ml}$, $47.7 \mathrm{mmol}, 2.0$ Äq.) wird die Reaktionslösung auf RT gebracht und über Nacht gerührt. Sobald die DC-Kontrolle kein Restalkohol mehr anzeigt, wird die organische Phase mit 0.1M Natriumhydrogencarbonat, Wasser und einer gesättigten NaClLösung extrahiert und über Magnesiumsulfat getrocknet. Das Rohprodukt wird säulenchromatographisch über Silicagel (Hexan/Ethylacetat, 10:1) gereinigt. Das gewünschte Produkt wird als farbloses Öl erhalten.

${ }^{1} \mathbf{H}-\mathbf{N M R}\left(200 \mathrm{MHz}, \mathrm{CDCl}_{3}\right): \delta=2.17\left(\mathrm{~s}, 3 \mathrm{H}, \mathrm{CH}_{3}\right), 6.36(\mathrm{~d}, J=6.9 \mathrm{~Hz}, 1 \mathrm{H}$, $\mathrm{C}(\mathrm{OAc}) \mathrm{H}), 6.48(\mathrm{dd}, J=6.9 \mathrm{~Hz}, J=15.4 \mathrm{~Hz}, 1 \mathrm{H}, \mathrm{CH}), 6.69$ (d, $J=15.4 \mathrm{~Hz}, 1 \mathrm{H}, \mathrm{CH})$, 7.46-7.30 (m, 10H, $\left.\mathrm{CH}^{A r}\right) \mathrm{ppm}$.

DC: (Silicagel; Hexan/Ethylacetat; 10:1) $\mathrm{R}_{\mathrm{f}}=0.41$.

\section{Synthese von (E)-Dimethyl-2-(1,3-Diphenylallyl)malonat}<smiles>CC(=O)OC(/C=C/c1ccccc1)c1ccccc1</smiles><smiles>CCC(C)C(C(=O)OC)C(C)(C)C</smiles><smiles>COC(=O)C(C(=O)OC)[C@H](/C=C/c1ccccc1)c1ccccc1</smiles>

Basierend auf [142]

Der Katalysator (0.02 mmol, 0.02 Äq.) wird in THF gelöst $(2 \mathrm{ml})$ und mit einer Lösung von (rac)-(E)-1,3-Diphenylallylacetat (252mg, 1.00mmol, 1.00Äq.) in THF 
$(1 \mathrm{ml})$ versetzt. In einem weiteren Kolben wird Dimethylmalonat $(0.34 \mathrm{ml}, 396 \mathrm{mg}$, $3.00 \mathrm{mmol}, 3.00 \mathrm{Äq}$ ) in THF (1 ml) gelöst und Natriumhydrid $(72 \mathrm{mg}, 3.00 \mathrm{mmol}$, 3.00Äq.) langsam zugegeben. Der daraus resultierende Feststoff wird der Katalysatorlösung zugegeben. Die Reaktionslösung wird bei der gewünschten Temperatur (RT und $45^{\circ} \mathrm{C}$ ) gerührt und der Verlauf der Reaktion durch DC-Kontrolle überwacht. Nach geeigneter Reaktionsdauer wird die Lösung mit Diethylether $(20 \mathrm{ml})$ verdünnt und mit einer gesättigten Ammoniumchloridlösung (3 x 20 ml) gewaschen. Die organische Phase wird über Magnesiumsulfat getrocknet und die Umwandlung ${ }^{1} \mathrm{H}-\mathrm{NMR}$ spektroskopisch überprüft. 



\section{Kapitel 7}

\section{Kristallstrukturdaten}

Die Rontgenstrukturanalysen wurden mit einem Stoe IPDS II-Gerät mit graphitmonochromatisierter Mo- $\mathrm{K}_{\alpha}$-Strahlung $(\lambda=0.71073 \AA)$ durchgefuhrt. Die Messwerte wurden bezüglich Lorentz- und Polarisationseffekten korrigiert. Zusätzlich wurden Absorptionskorrekturen vorgenommen. Das Lösen und die Verfeinerung erfolgten mit den Programmen SHELXS-97 und SHELXL-97. [143-145] Für die graphische Darstellung der Strukturen wurde das Programm DIAMOND 3.0 verwendet. 


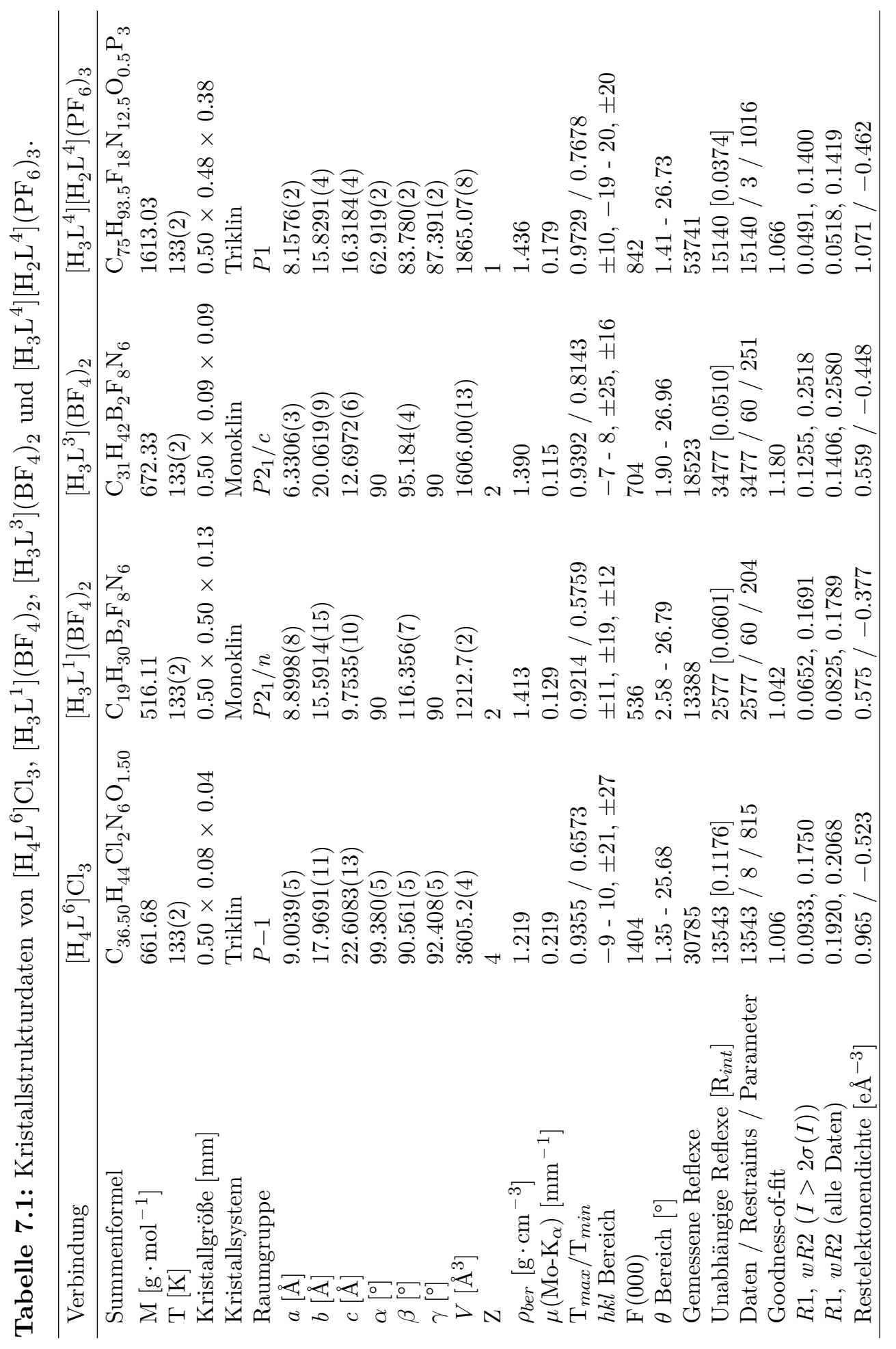




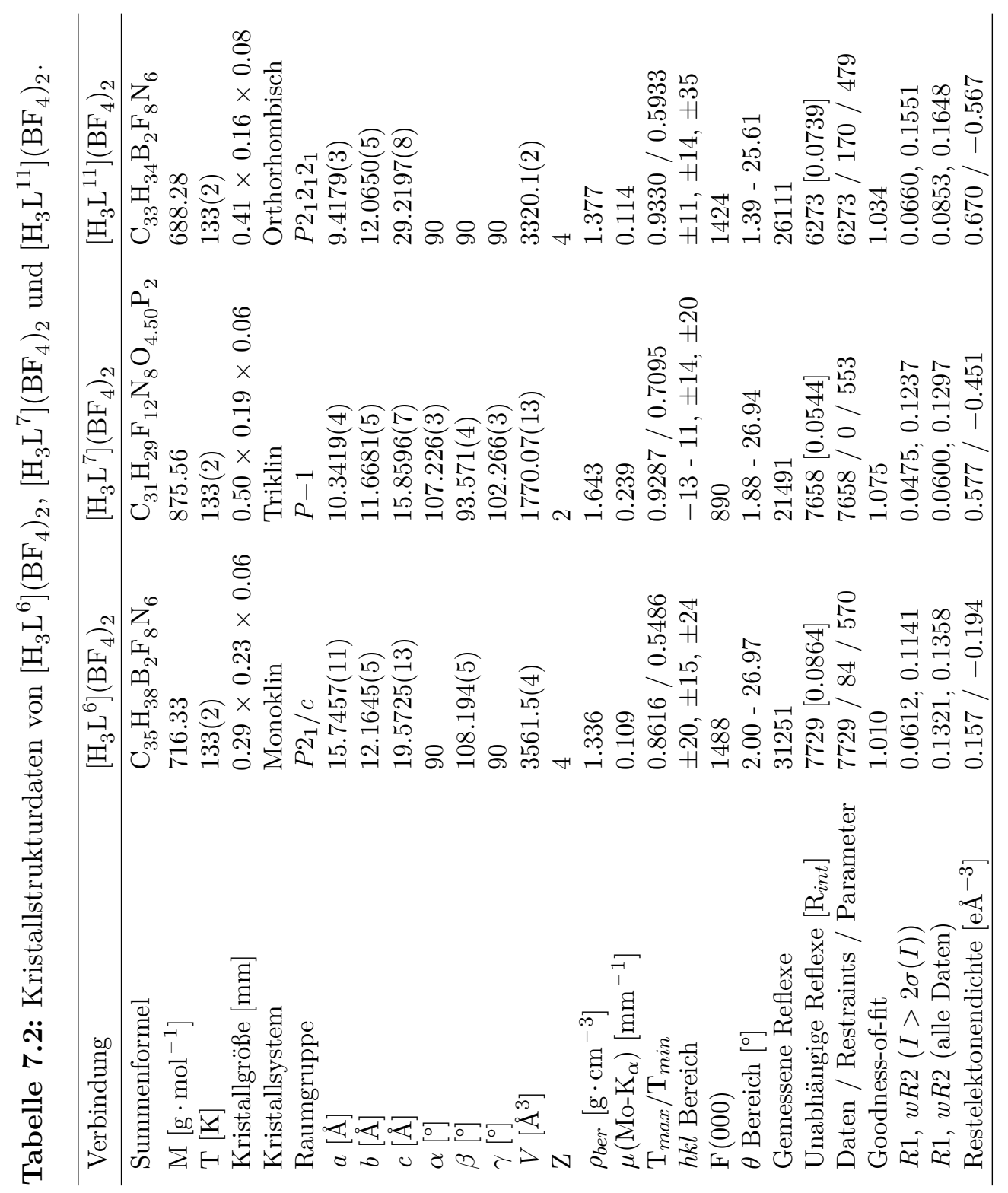




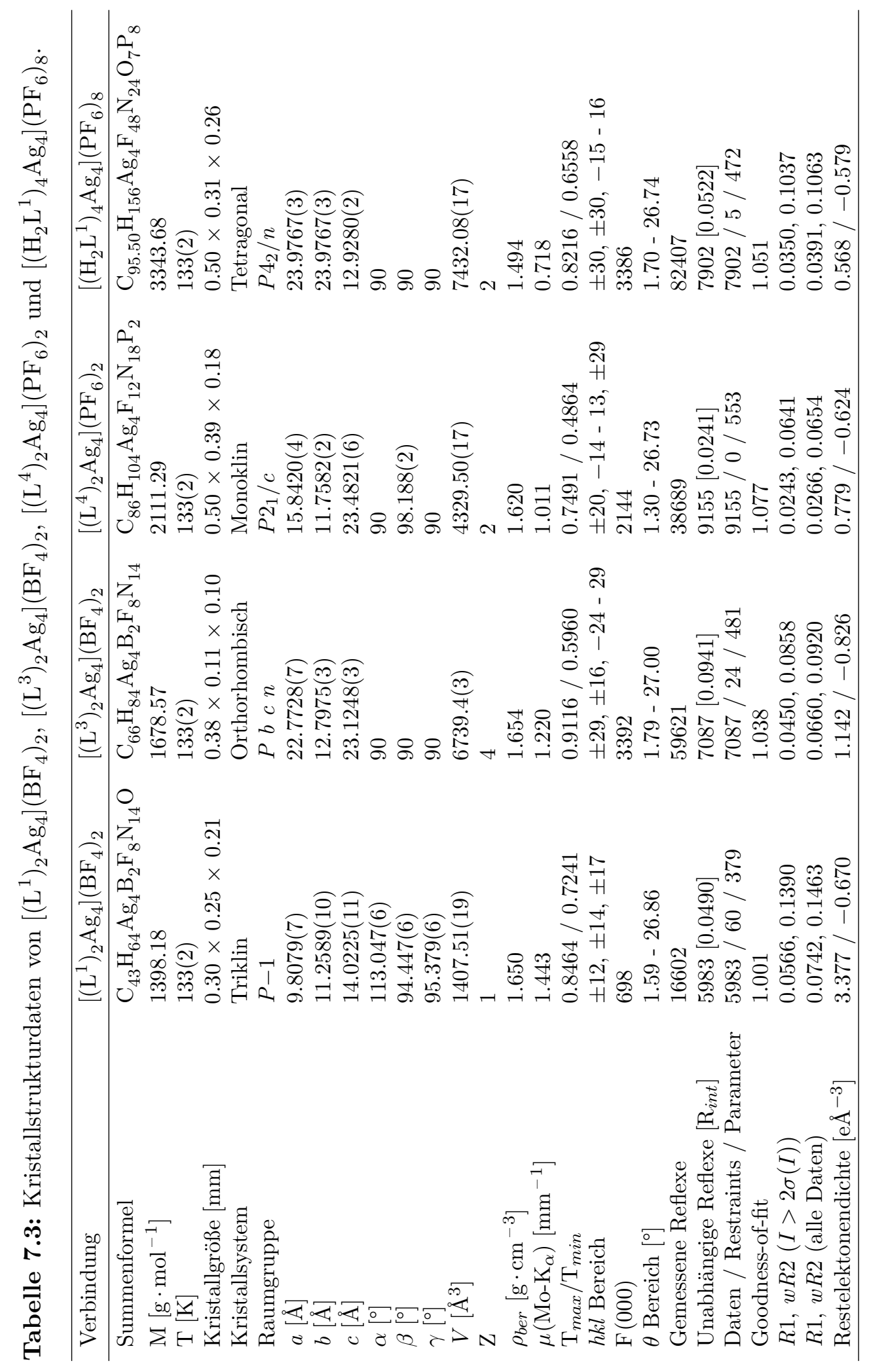




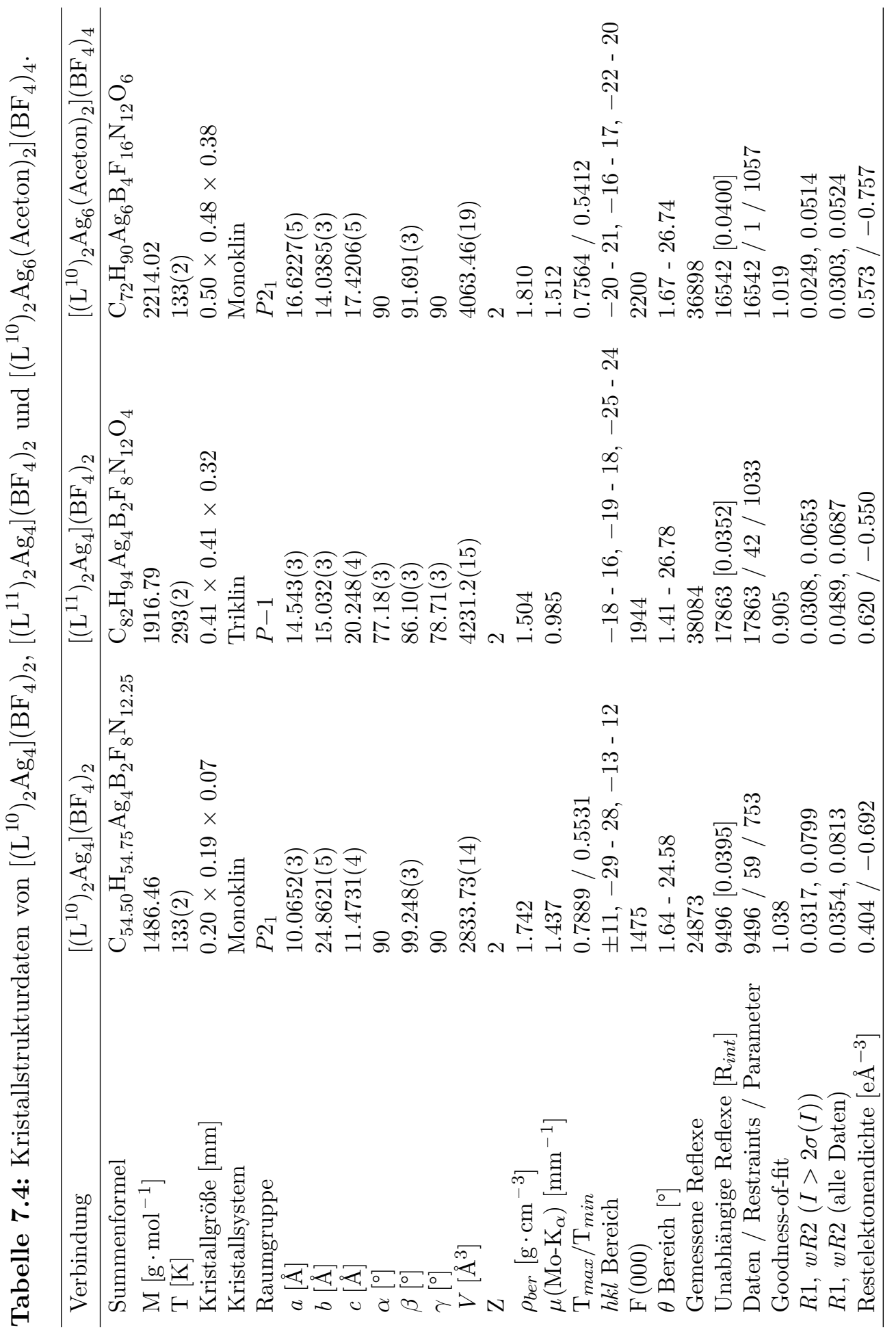




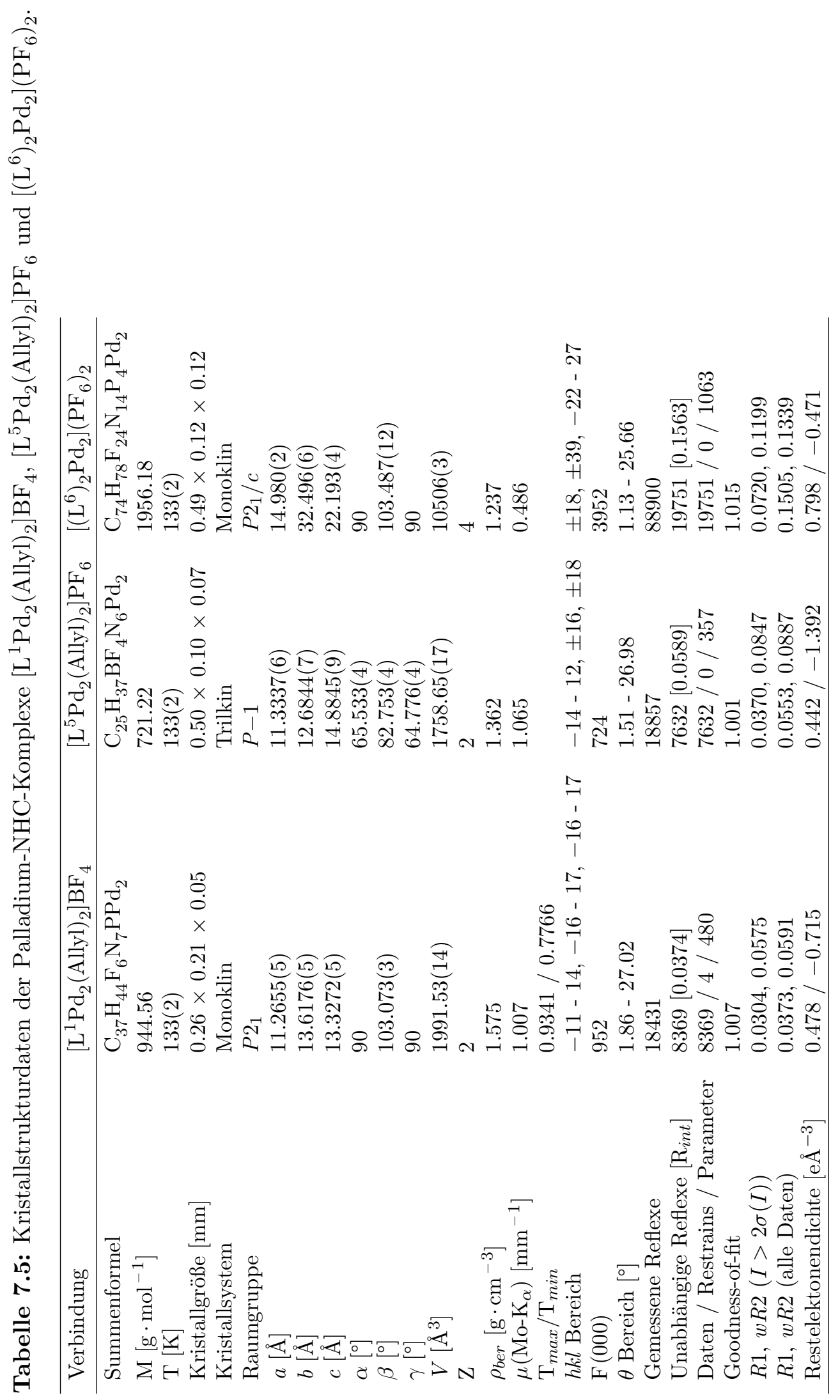


Teil III

Anhang 



\section{Literaturverzeichnis}

[1] D. Bourissou, O. Guerret, F. P. Gabbai, G. Bertrand, Chem. Rev. 2000, 100, 39-91. $3,4,5$

[2] W. A. Herrmann, C. Köcher, Angew. Chem. 1997, 109, 2256-2282. 4, 5

W. A. Herrmann, C. Köcher, Angew. Chem. Int. Ed. 1997, 36, 2162-2187.

[3] A. J. Arduengo, R. Krafczyk, Chemie in unserer Zeit 1998, 32, 6-14. 4, 6

[4] F. E. Hahn, M. C. Jahnke, Angew. Chem. 2008, 120, 3166-3216. 4

F. E. Hahn, M. C. Jahnke, Angew. Chem. Int. Ed. 2008, 47, 3122-3172.

[5] R. Hoffmann, G. D. Zeiss, G. W. Vandine, J. Am. Chem. Soc. 1968, 90, 1485-1499. 4

[6] L. Pauling, J. Chem. Soc., Chem. Commun. 1980, 688-689. 4

[7] R. A. Moss, C. B. Mallon, C. T. Ho, J. Am. Chem. Soc. 1977, 99, 4105-4110. 4

[8] B. C. Gilbert, D. Griller, A. S. Nazran, J. Org. Chem. 1985, 50, 4738-4742. 5

[9] J. B. Dumas, E. Piligot, Ann. Chim. Phys. 1835, 58, 5. 6

[10] A. Geuther, Ann. Chem. Pharm. 1862, 123, 121. 6

[11] U. Nef, Justus Liebigs Ann. Chem. 1897, 298, 202. 6

[12] L. Tshugajeff, M. Seanawy-Grigorjewa, A. Posnjak, Z. Anorg. Allg. Chem. 1925, 148, 37-42. 6

[13] H.-W. Wanzlick, E. Schikora, Angew. Chem. 1960, 72, 494-495. 6

[14] D. M. Lemal, R. A. Lovald, K. I. Kawano, J. Am. Chem. Soc. 1964, 86, 2518-2519. 7

[15] K. Öfele, J. Organomet. Chem. 1968, 12, P42. 7, 15 
[16] H.-W. Wanzlick, H.-J. Schönherr, Angew. Chem. Int. Ed. 1968, 7, 141-142. 7,15

H.-W. Wanzlick, H.-J. Schönherr, Angew. Chem. 1968, 80, 154.

[17] D. J. Cardin, B. Cetinkay, M. F. Lappert, L. Manojlov, K. W. Muir, J. Chem. Soc. D 1971, 400-401. 7

[18] M. F. Lappert, J. Organomet. Chem. 1988, 358, 185-214. 7, 17

[19] A. J. Arduengo, R. L. Harlow, M. Kline, J. Am. Chem. Soc. 1991, 113, 361363. 7

[20] W. A. Herrmann, M. Elison, J. Fischer, C. Köcher, G. R. J. Artus, Angew. Chem. 1995, 10\%, 2602-2605. 7, 24

W. A. Herrmann, M. Elison, J. Fischer, C. Köcher, G. R. J. Artus, Angew. Chem., Int. Ed. Engl. 1995, 34, 2371-2374.

[21] E. O. Fischer, A. Maasbol, Angew. Chem. 1964, 76, 645. 8

E. O. Fischer, A. Maasbol, Angew. Chem. Int. Ed. 1964, 3, 580-581.

[22] R. R. Schrock, US 3988332, 1976. 8

[23] I. Feinstein-Jaffe, S. F. Pedersen, R. R. Schrock, J. Am. Chem. Soc. 1983, $105,7176-7177.8$

[24] A. F. Holleman, N. Wiberg, Lehrbuch der Anorganischen Chemie, Walter de Gruyter, Berlin, 1995. 8

[25] W. A. Herrmann, M. K. Denk, J. Behm, W. Scherer, F. Klingan, H. Bock, B. Solouki, M. Wagner, Angew. Chem. 1992, 104, 1489-1492. 9

W. A. Herrmann, M. K. Denk, J. Behm, W. Scherer, F. Klingan, H. Bock, B. Solouki, M. Wagner, Angew. Chem. Int. Ed. 1992, 31, 1485-1488.

[26] J. C. Green, R. G. Scurr, P. L. Arnold, F. Geoffrey, N. Cloke, Chem. Commun. 1997, 1963-1964. 9

[27] J. C. Green, B. J. Herbert, Dalton Trans. 2005, 1214-1220. 9

[28] J. Schwarz, V. P. W. Böhm, M. Gardiner, M. Grosche, W. Herrmann, W. Hieringer, G. Raudaschl-Sieber, Chem. Eur. J. 2000, 6, 1773-1780. 10

[29] W. A. Herrmann, C. W. Kohlpaintner, Angew. Chem. 1993, 105, 1588-1609. 10

Angew. Chem. Int. Ed. 1993, 32, 1524-1544. 
[30] L. G. Bonnet, R. E. Douthwaite, B. M. Kariuki, Organometallics 2003, 22, 4187-4189. 10

[31] A. Flahaut, S. Roland, P. Mangeney, Tetrahedron: Asymmerty 2007, 18, 229236. 10

[32] C. .-C. Lee, W. C. Ke, K.-T. Chan, C. L. Lai, C. H. Hu, H. M. Lee, Chem.-Eur. J. 2007, 13, 582-591. 10

[33] N. Toselli, D. Martin, G. Buono, Org. Lett. 2008, 10, 1453-1456. 10

[34] Y. B. Zhou, W. Z. Chen, Organometallics 2007, 26, 2742-2746. 11, 19, 20, $22,24,46$

[35] U. J. Scheele, M. John, S. Dechert, F. Meyer, Eur. J. Inorg. Chem. 2008, 373-377. 24, 29, 44, 49, 50, 51, 53, 69, 74, 82, 87, 88

[36] S.-J. Jeon, R. M. Waymouth, Dalton Trans. 2008, 437-439. 57

[37] Y. Zhou, Z. Xi, W. Chen, D. Wang, Organometallics 2008, 27, 5911-5920. $11,20,57$

[38] T. Schenck, C. Milne, J. Sawyer, B. Bosnich, Inorg. Chem. 1985, 24, 23342337. 11,25

[39] J. C. Röder, F. Meyer, H. Pritzkow, Organometallics 2001, 20, 811-817. 11, 69

[40] E. Büchner, C. v. Heide, Ber. Dtsch. Chem. Ges. 1902, 35, 31-34. 12

[41] P. C. Myhre, C. T. Maxey, D. C. Bebout, S. H. Swedberg, B. L. Petersen, J. Org. Chem. 1990, 55, 3417-3421.

[42] A. Sachse, L. Penkova, G. Nöel, S. Dechert, O. A. Varzatskii, I. O. Fritsky, F. Meyer, Synthesis 2008, 5, 800-806. 33

[43] M. Stollenz, C. Gross, F. Meyer, Chem. Commun. 2008, 1744-1746. 12, 69

[44] A. A. Gridnev, I. M. Mihaltseva, Synth. Commun. 1994, 24, 1547-1555. 12, 14,69

[45] M. G. Gardiner, W. A. Herrmann, C.-P. Reisinger, J. Schwarz, M. Spiegler, J. Organomet. Chem. 1999, 572, 239-247.

[46] M. C. Perry, X. H. Cui, M. T. Powell, D. R. Hou, J. H. Reibenspies, K. Burgess, J. Am. Chem. Soc. 2003, 125, 113-123. 12, 69

[47] A. J. Arduengo III, US 5077414, 1991. 12 
[48] J. Liu, J. Chen, J. Zhao, Y. Zhao, L. Li, H. Zhang, Synthesis 2003, 2661-2666. 13

[49] U. J. Scheele, S. Dechert, F. Meyer, Tetrahedron Lett. 2007, 48, 8366-8370. 13,35

[50] G. Matolcsy, P. Bartók, Acta Phytopathol. Acad. Sci. Hung. 1978, 13, 223225. 14

[51] M. E. Gonzalez, B. Alarcon, P. Cabildo, R. M. Claramunt, D. Sanz, J. Elguero, Eur. J. Med. Chem. 1985, 20, 359-362.

[52] G. F. Raenko, N. I. Korotkikh, T. M. Pekhtereva, O. P. Shvaika, Russ. J. Org. Chem. 2001, 3\%, 1153-1157. 14

[53] A. L. Johnson, J. C. Kauer, D. C. Sharma, R. I. Dorfman, J. Med. Chem. 1969, 12, 1024-1028. 15

[54] S. Seko, K. Miyake, N. Kawamura, J. Chem. Soc., Perkin Trans. 1 1999, 1437-1444. 15

[55] E. A. B. Kantchev, C. J. O‘Brien, M. G. Organ, Angew. Chem. 2007, 119, 2824-2870. 15, 24

E. A. B. Kantchev, C. J. O‘Brien, M. G. Organ, Angew. Chem. Int. Ed. 2007, 46, 2768-2813.

[56] T. Weskamp, V. P. W. Bohm, W. A. Herrmann, J. Organomet. Chem. 2000, $600,12-22.15$

[57] A. J. Arduengo, R. Krafczyk, R. Schmutzler, H. A. Craig, J. R. Goerlich, W. J. Marshall, M. Unverzagt, Tetrahedron 1999, 55, 14523-14534. 15

[58] M. H. Voges, C. Rømming, M. Tilset, Organometallics 1999, 18, 529-533. 15

[59] H. M. J. Wang, I. J. B. Lin, Organometallics 1998, 17, 972-975. 17, 36

[60] M. F. Lappert, J. Organomet. Chem. 2005, 690, 5467-5473. 17

[61] B. Cetinkaya, P. B. Hitchcock, M. F. Lappert, D. B. Shaw, K. Spyropoulos, N. J. W. Warhurst, J. Organomet. Chem. 1993, 459, 311-317. 17

[62] A. J. Arduengo, H. V. R. Dias, J. C. Calabrese, F. Davidson, Organometallics 1993, 12, 3405-3409. 18

[63] A. A. D. Tulloch, S. Winston, A. A. Danopoulos, G. Eastham, M. B. Hursthouse, Dalton Trans. 2003, 699-708. 18 
[64] O. Guerret, S. Sole, H. Gornitzka, M. Teichert, G. Trinquier, G. Bertrand, J. Am. Chem. Soc. 1997, 119, 6668-6669. 18

[65] O. Guerret, S. Sole, H. Gornitzka, G. Trinquier, G. Bertrand, J. Organomet. Chem. 2000, 600, 112-117. 18

[66] A. A. D. Tulloch, A. A. Danopoulos, S. Winston, S. Kleinhenz, G. Eastham, J. Chem. Soc., Dalton Trans. 2000, 4499-4506. 18

[67] J. C. Garrison, W. J. Youngs, Chem. Rev. 2005, 105, 3978-4008. 19

[68] I. J. B. Lin, C. S. Vasam, Coord. Chem. Rev. 2007, 251, 642-670.

[69] J. C. Y. Lin, R. T. W. Huang, C. S. Lee, A. Bhattacharyya, W. S. Hwang, I. J. B. Lin, Chem. Rev. 2009, 109, 3561-3598. 19, 22

[70] D. Pugh, A. Boyle, A. A. Danopoulos, Dalton Trans. 2008, 1087-1094. 19

[71] F. J. B. Dominique, H. Gornitzka, C. Hermmert, J. Organomet. Chem. 2008, 693, 579-583. 19

[72] J. S. Ye, S. W. Jin, W. Z. Chen, H. Y. Qiu, Inorg. Chem. Commun. 2008, 11, 404-408. 19, 22

[73] V. J. Catalano, M. A. Malwitz, Inorg. Chem. 2003, 42, 5483-5485. 19

[74] V. J. Catalano, A. O. Etogo, J. Organomet. Chem. 2005, 690, 6041-6050. 22, 44

[75] Z. Xi, X. Zhang, W. Chen, S. Fu, D. Wang, Organometallics 2007, 26, 66366642. 19

[76] J. C. Garrison, R. S. Simons, W. G. Kofron, C. A. Tessier, W. J. Youngs, Chem. Commun. 2001, 1780-1781. 19

[77] J. C. Garrison, R. S. Simons, C. A. Tessier, W. J. Youngs, J. Organomet. Chem. 2003, 673, 1-4. 19

[78] B. Liu, W. Chen, S. Jin, Organometallics 2007, 26, 3660-3667. 19, 44

[79] U. J. Scheele, M. Georgiou, M. John, S. Dechert, F. Meyer, Organometallics 2008, 27, 5146-5151. 19, 21, 39, 40

[80] H. H. Murray, R. G. Raptis, J. P. Fackler, Jr., Inorg. Chem. 1988, 27, 26-33. 19,40

[81] F. Meyer, A. Jacobi, L. Zsolnai, Chem. Ber./Recueil 1997, 130, 1441-1447. 
[82] H. V. R. Dias, H. V. K. Diyabalanage, Polyhedron 2006, 25, 1655-1661.

[83] D. M. M. Krishantha, C. S. P. Gamage, Z. A. Schelly, H. V. R. Dias, Inorg. Chem. 2008, 47, 7065-7067. 19, 40

[84] G. Yang, R. G. Raptis, Inorg. Chim. Acta 2007, 360, 2503-2506. 20, 40

[85] H. Schmidbaur, Gold Bull. 2000, 33, 3-10. 22

[86] Y. B. Zhou, X. M. Zhang, W. Z. Chen, H. Y. Qiu, J. Organomet. Chem. 2008, 693, 205-215. 22, 36

[87] A. Liu, X. Zhang, W. Chen, H. Qiu, Inorg. Chem. Commun. 2008, 11, 11291132. 22

[88] G. Emig, Chemie in unserer Zeit 1987, 21, 128-137. 22, 23

[89] M. Röper, Chemie in unserer Zeit 2006, 40, 126-135. 23

[90] J. K. Lee, M. C. Kung, H. H. Kung, Top. Catal. 2008, 49, 136-144. 23, 25

[91] K. Muñiz, Chemie in unserer Zeit 2006, 40, 112-124. 23

[92] M. S. Viciu, S. P. Nolan, Top. Organomet. Chem. 2005, 14, 241-278. 24

[93] C. Yang, H. M. Lee, S. P. Nolan, Org. Lett. 2001, 3, 1511-1514. 24

[94] C. W. K. Gstöttmayr, V. P. W. Böhm, E. Herdtweck, M. Grosche, W. A. Herrmann, Angew. Chem. 2002, 114, 1421-1423. 24

C. W. K. Gstöttmayr, V. P. W. Böhm, E. Herdtweck, M. Grosche, W. A. Herrmann, Angew. Chem. Int. Ed. 2002, 41, 1363-1365.

[95] C. Yang, S. Nolan, Organometallics 2002, 21, 1020-1022. 24

[96] M. Eckhardt, G. C. Fu, J. Am. Chem. Soc. 2003, 125, 13642-13643. 24

[97] G. A. Grasa, S. P. Nolan, Org. Lett. 2001, 3, 119-122. 24

[98] V. P. W. Böhm, T. Weskamp, C. W. K. Gstöttmayr, W. A. Herrmann, Angew. Chem. 2000, 112, 1672-1674. 24

V. P. W. Böhm, T. Weskamp, C. W. K. Gstöttmayr, W. A. Herrmann, Angew. Chem. Int. Ed. 2000, 39, 1602-1604.

[99] H. M. Lee, D. C. Smith Jr., Z. He, E. D. Stevens, C. S. Yi, S. P. Nolan, Organometallics 2001, 20, 794-797. 24

[100] J. W. Sprengers, J. Wassenaar, N. D. Clement, K. J. Cavell, C. J. Elsevier, Angew. Chem. 2005, 117, 2062-2065. 24

J. W. Sprengers, J. Wassenaar, N. D. Clement, K. J. Cavell, C. J. Elsevier, Angew. Chem. Int. Ed. 2005, 44, 2026-2029. 
[101] A. C. Chen, L. Ren, A. Decken, C. M. Crudden, Organometallics 2000, 19, 3459-3461. 24

[102] S. Diez-Gonzalez, E. D. Stevens, N. M. Scott, J. L. Petersen, S. P. Nolan, Chem.-Eur. J. 2008, 14, 158-168. 24

[103] S. C. Zinner, M. Zhang-Presse, W. A. Herrmann, F. E. Kühn, Z. Naturforsch., B 2009, 64, 1607-1611. 24

[104] W. A. Herrmann, Angew. Chem. 2002, 114, 1342-1363. 24

W. A. Herrmann, Angew. Chem. Int. Ed. 2002, 41, 1290-1309.

[105] A. Fürstner, Angew. Chem. 2000, 112, 3140-3172. 24

A. Fürstner, Angew. Chem. Int. Ed. 2000, 39, 3013-3043.

[106] A. Fürstner, L. Ackermann, B. Gabor, Goddard, C. W. Lehmann, R. Mynott, F. Stelzer, O. R. Thiel, Chem.-Eur. J. 2001, 7, 3236-3253.

[107] T. M. Trnka, R. H. Grubbs, Acc. Chem. Res. 2001, 34, 18-29. 24

[108] S. T. Nguyen, L. K. Johnson, R. H. Grubbs, J. Am. Chem. Soc. 1992, 114, 3974-3975. 24

[109] Y. Chauvin, Angew. Chem. 2006, 118, 3824-3831. 24

Y. Chauvin, Angew. Chem. Int. Ed. 2006, 45, 3740-3748.

[110] R. R. Schrock, Angew. Chem. 2006, 118, 3832-3844.

R. R. Schrock, Angew. Chem. Int. Ed. 2006, 45, 3748-3759.

[111] R. H. Grubbs, Angew. Chem. 2006, 118, 3845-3850. 24

R. H. Grubbs, Angew. Chem. Int. Ed. 2006, 45, 3760-3765.

[112] H. Steinhagen, M. Reggelin, G. Helmchen, Angew. Chem. 1997, 109, 21992202. 25,28

H. Steinhagen, M. Reggelin, G. Helmchen, Angew. Chem., Int. Ed. Engl. 1997, 36, 2108-2110.

[113] B. M. Trost, M. L. Crawley, Chem. Rev. 2003, 103, 2921-2943.

[114] B. M. Trost, D. L. v. Vranken, Chem. Rev. 1996, 96, 395-422. 25

[115] P. S. Pregosin, R. Salzmann, Coord. Chem. Rev. 1996, 155, 35-68. 25

[116] C. Foces-Foces, I. Alkorta, J. Elguero, Acta Crystallogr. 2000, B56, 10181028. 33 
[117] U. J. Scheele, S. Dechert, F. Meyer, Inorg. Chim. Acta 2006, 359, 4891-4900. 33,35

[118] T. Steiner, Acta Crystallogr. 1998, B54, 456-463. 35

[119] P. Pyykkö, Chem. Rev. 1997, 97, 597-636. 37

[120] H. Schmidbaur, A. Schier, Chem. Soc. Rev. 2008, 37, 1931-1951.

[121] M. Jansen, Angew. Chem. 1987, 99, 1136-1138.

M. Jansen, Angew. Chem., Int. Ed. Engl. 1987, 26, 1098-1110. 37, 39

[122] C. M. Che, S. W. Lai, Coord. Chem. Rev. 2005, 249, 1296-1309. 37, 39

[123] V. J. Catalano, M. A. Malwitz, A. O. Etogo, Inorg. Chem. 2004, 43, 57145724. 44

[124] U. J. Scheele, Entwicklung von Übergangsmetallkomplexen mit neuartigen Pyrazol-NHC- und Pyridazin-NHC-Hybridliganden, 1. Auflage, Cuvillier Verlag Göttingen, 2008. 46

[125] B. E. Ketz, A. P. Cole, R. M. Waymouth, Organometallics 2004, 23, 28352837. 51

[126] S. K. Schneider, J. Schwarz, G. D. Frey, E. Herdtweck, W. A. Herrmann, J. Organomet. Chem. 2007, 692, 4560-4568. 56

[127] C. D. Abernethy, A. H. Cowley, R. A. Jones, J. Organomet. Chem. 2000, 596, 3-5. 58, 91

[128] A. O. Larsen, W. Leu, C. Nieto Oberhuber, J. E. Campbell, A. H. Hoveyda, J. Am. Chem. Soc. 2004, 126, 11130-11131. 59

[129] H. E. Gottlieb, V. Kotlyar, A. Nudelman, J. Org. Chem. 1997, 62, 7512-7515. 67

[130] G. R. Fulmer, A. J. M. Miller, N. H. Sherden, H. E. Gottlieb, A. Nudelman, B. M. Stoltz, J. E. Bercaw, K. I. Goldberg, Organometallics 2010, 29, 21762179. 67

[131] A. Bax, R. H. Griffey, B. L. Hawkins, J. Magn. Reson. 1983, 301-315. 67

[132] R. K. Harris, E. D. Becker, S. M. Cabral de Menezes, R. Goodfellow, P. Granger, Pure Appl. Chem. 2001, 73, 1795-1818. 68

[133] M. Monroe, Molecular Weight Calculator, Version 6.37, 2004. 68 
[134] W. A. Herrmann, C. Köcher, L. J. Goossen, G. R. J. Artus, Chem.-Eur. J. 1996, 2, 1627-1636. 69

[135] J. R. Doyle, P. E. Slade, H. B. Jonassen, Inorg. Synth. 1960, 6, 216-219. 69

[136] G. Brauer, Handbuch der Präparativen Anorganischen Chemie in drei Bänden, Bd. 3, 3. umgearbeitete Auflage, Enke: Stuttgart, 1981. 69

[137] B. L. Shaw, Proc. Chem. Soc. 1960, 247. 69

[138] W. T. Dent, R. Long, A. J. Wilkinson, J. Chem. Soc. 1964, 1585-1588. 69

[139] Y. Tatsuno, T. Yoshida, S. Otsuka, Inorg. Synth. 1988, 28. 69

[140] G. Brauer, Handbuch der Prp̈arativen Anorganischen Chemie in drei Bänden, Bd. 3, 3. umgearbeitete Auflage, Enke: Stuttgart, 1981. 69

[141] I. D. G. Watson, A. K. Yudin, J. Am. Chem. Soc. 2005, 127, 17516-17529. 92

[142] U. Leutenegger, G. Umbricht, C. Fahrni, P. von Matt, A. Pfaltz, Tetrahedron 1992, 48, 2143-2156. 92

[143] G. M. Sheldrick, SHELXS-97: Program for Crystal Structure Solution, University of Goettingen, 1997. 95

[144] G. M. Sheldrick, SHELXL-97: Program for Crystal Structure Refinement, University of Goettingen, 1997.

[145] X-RED, Crystal Optimisation for Numerical Absorption Correction, Stoe \& Cie GmbH, Darmstadt (Germany), 2002. 95 



\section{Symbolverzeichnis}

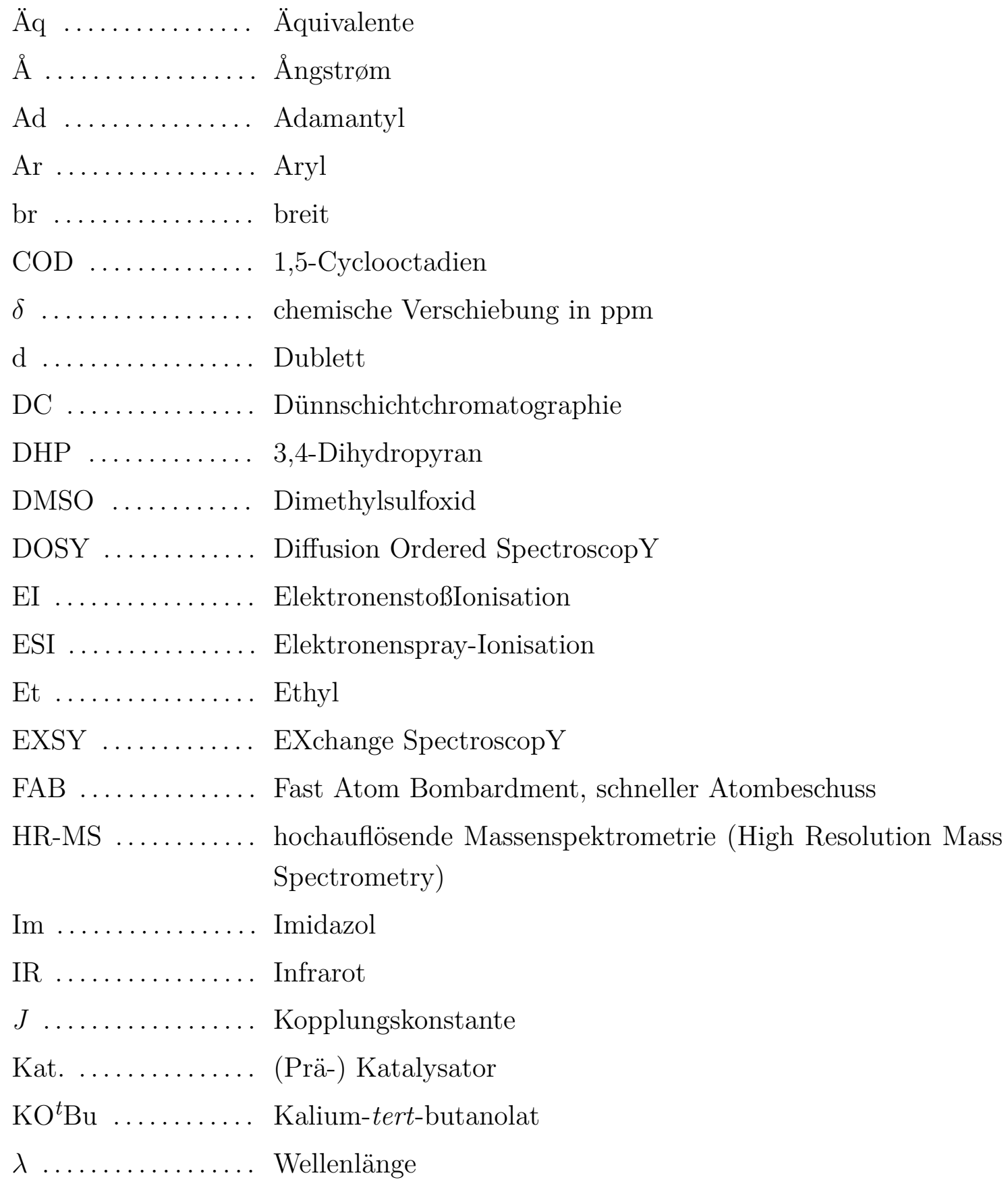


LAH $\ldots \ldots \ldots \ldots$ Lithiumaluminiumhydrid

$\mathrm{L} \ldots \ldots \ldots \ldots \ldots$ Ligand

$m / z \ldots \ldots \ldots \ldots$ Masse-zu-Ladung Verhältnis

MeCN ........... Acetonitril

$\mathrm{MeOH}$........... Methanol

Mes $\ldots \ldots \ldots \ldots$. Mesityl $=$ 2,4,6-Trimethylphenyl

MS ............ Massenspektrometrie

NHC $\ldots \ldots \ldots \ldots . N$-heterozyklisches Carben

NMR .......... magnetische Kernresonanz (Nuclear Magnetic Resonance)

NOE ........... Nuclear Overhauser Effect

OAc ............. Acetat

$\mathrm{Pz}^{*} \ldots \ldots \ldots \ldots$ unterschiedlich substituiertes Pyrazolat-Anion

$\mathrm{Pz}^{\mathrm{H}}{ }^{-T H P} \ldots \ldots \ldots$ 3,5-Bis(chlormethyl)-1-(tetrahydropyran-2-yl)-1 $H$-pyrazol

$\mathrm{Pz}^{\mathrm{Ph}}{ }_{-\mathrm{THP}} \ldots \ldots \ldots$ 3,5-Bis(chlormethyl)-1-(tetrahydropyran-2-yl)-4-phenyl-1Hpyrazol

$\mathrm{Ph} \ldots . . . . . .$. Phenyl

$\operatorname{ppm} \ldots \ldots \ldots \ldots$ parts per million

$\mathrm{Pz} \ldots \ldots \ldots \ldots$. Pyrazol

q $\ldots \ldots \ldots \ldots$........ Quartett

R ............ beliebiger chemischer Rest

RT ........... Raumtemperatur

SA $\ldots . . \ldots \ldots$ Seitenarm (substituiertes Imidazol)

S $\ldots \ldots \ldots \ldots$. Singulett

$t \mathrm{Bu} \quad \ldots \ldots \ldots \ldots$ tert-Butyl

THF $\ldots . . . \ldots .$. Tetrahydrofuran

THP ........... Tetrahydropyran

t $\ldots \ldots \ldots \ldots \ldots$ Triplett

$\mathrm{UV} /$ Vis ......... Ultraviolett/sichtbar

VE $\ldots \ldots \ldots \ldots \ldots$ Valenzelektron(e) 


\section{Abbildungsverzeichnis}

1.1 Struktur von Singulett- (I) und Triplett-Carbenen (II) . . . . . . . . 3

1.2 Einfluss von Substituenten mit induktiven und mesomeren Effekten. 5

1.3 Mesomere Grenzstrukturen von Imidazolyl-2-ylidenen. . . . . . . . . 5

1.4 Beispiel eines Fischer- (rechts) und eines Schrock-Carbenkomplexes (links). . . . . . . . . . . . . . . . . . 9

2.1 Koordinationsmöglichkeiten von Silber-NHC-Komplexen bisher. . . 20

2.2 Mögliche strukturelle Motive A und B von Münzmetall-PyrazolatKomplexen. . . . . . . . . . . . . . . . . . . . 21

2.3 Unterschiedliche Strukturen von mehrkernigen pyrazolverbrückten Silber(I)-NHC-Komplexen C und D. . . . . . . . . . . . . . . . . 21

2.4 Grubbs-Metathesekatalysatoren der ersten (XIII) und zweiten (XIV) Generation. . . . . . . . . . . . . . . . . .

3.1 Allgemeines Bindungsmodell der Pyrazol-NHC-Hybridliganden. . . 28

4.1 Festkörperstruktur von $\left[\mathrm{H}_{4} \mathrm{~L}^{6}\right] \mathrm{Cl}_{3} \ldots \ldots \ldots \ldots$

4.2 Festkörperstruktur von $\left[\mathrm{H}_{3} \mathrm{~L}^{1}\right]\left(\mathrm{BF}_{4}\right)_{2}$ und Konstitution von

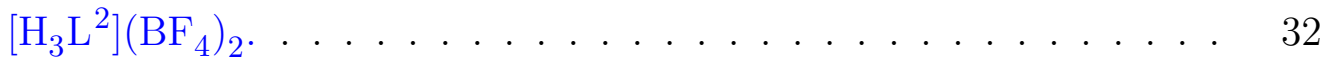

4.3 Festkörperstruktur von $\left[\mathrm{H}_{3} \mathrm{~L}^{4}\right]\left[\mathrm{H}_{2} \mathrm{~L}^{4}\right]\left(\mathrm{PF}_{6}\right)_{3} \ldots \ldots \ldots 33$

4.4 Festkörperstruktur von $\left[\mathrm{H}_{3} \mathrm{~L}^{11}\right]\left(\mathrm{BF}_{4}\right)_{2} \ldots \ldots \ldots \ldots$

4.5 Festkörperstrukturen der $\mathrm{Ag}(\mathrm{I})-\mathrm{NHC}-K o m p l e x e\left[\left(\mathrm{~L}^{1}\right)_{2} \mathrm{Ag}_{4}\right]\left(\mathrm{BF}_{4}\right)_{2}$ und $\left[\left(\mathrm{L}^{2}\right)_{2} \mathrm{Ag}_{4}\right]\left(\mathrm{BF}_{4}\right)_{2} \ldots \ldots \ldots . \ldots \ldots$

4.6 Festkörperstruktur von $\left[\left(\mathrm{H}_{2} \mathrm{~L}^{1}\right)_{4} \mathrm{Ag}_{4}\right]\left(\mathrm{PF}_{6}\right)_{8}$ und seitlicher Ansicht. . 41

4.7 Festkörperstruktur von $\left[\left(\mathrm{L}^{10}\right)_{2} \mathrm{Ag}_{4}\right]\left(\mathrm{BF}_{4}\right)_{2} \ldots \ldots \ldots \ldots$

4.8 Festkörperstruktur von $\left.\left[\left(\mathrm{L}^{10}\right)_{2} \mathrm{Ag}_{6} \text { (Aceton }\right)_{2}\right]\left(\mathrm{BF}_{4}\right)_{4} \ldots \ldots \ldots . . . \quad 45$

4.9 Absorptions- und Emissionsspektrum von $\left[\left(\mathrm{H}_{2} \mathrm{~L}^{1}\right)_{4} \mathrm{Ag}_{4}\right]\left(\mathrm{PF}_{6}\right)_{8} \ldots$. . 47

4.10 Festkörperstruktur von $\left[\mathrm{L}^{5} \mathrm{Pd}_{2}(\mathrm{Allyl})_{2}\right] \mathrm{PF}_{6}$. . . . . . . . . . . . 50

4.11 Festkörperstruktur von $\left[\mathrm{L}^{1} \mathrm{Pd}_{2}(\mathrm{Allyl})_{2}\right] \mathrm{BF}_{4} \ldots \ldots \ldots \ldots$

4.12 Festkörperstruktur von $\left[\left(\mathrm{HL}^{6}\right)(\mathrm{MeCN}) \mathrm{Pd}\right]_{2}\left(\mathrm{PF}_{6}\right)_{4} \ldots \ldots \ldots$ 
4.13 Bimetallischer Ni-NHC-Komplex für eine mögliche katalytische Anwendung. . . . . . . . . . . . . . . . . . 58

4.14 Ni-NHC-Komplex der Zusammensetzung $\left[\mathrm{L}_{2} \mathrm{Ni}_{2}\right] \ldots \ldots \ldots$

5.1 Verwendete Ligandsysteme. . . . . . . . . . . . . . . . 62 


\section{Verzeichnis der Schemata}

1.1 Erster Versuch Methylen aus Methanol zu isolieren. . . . . . . . . . 6

1.2 Darstellung eines intermediär auftretenden Methylen-Derivates. . . 6

1.3 Isolierung des ersten mit Stickstoffatomen stabilisierten PlatinCarbenkomplexes VI. . . . . . . . . . . . . . . . . . . 7

1.4 Darstellung des Tetraaminoethylen-Derivats IX durch $\alpha$ Eliminierung von VII. . . . . . . . . . . . . . . 7

1.5 Carben-Metallkomplexe nach Öfele (oben) und Wanzlick (unten). . 8

1.6 Synthese des ersten von Arduengo isolierten stabilen $N$ heterozyklischen Carbens X. . . . . . . . . . . . 8

2.1 Darstellung von 3,5-Bis(chlormethyl)-1-(tetrahydropyran-2-yl)-1Hpyrazol (6). . . . . . . . . . . . . . . . . . . . 12

2.2 Synthese von 3,5-Bis(chlormethyl)-1-(tetrahydropyran-2-yl)-4phenyl-1H-pyrazol (12). . . . . . . . . . . . . . . . . . 13

2.3 Gängige Syntheseroute der $N$-substituierten Imidazole. . . . . . . . 14

2.4 Alternative Synthese von 1-(1-Adamantyl)-1H-imidazol. . . . . . . . 14

2.5 Synthese von 1-(tert-Butyl)-1H-imidazol. . . . . . . . . . . . . . 15

2.6 In situ Deprotonierung eines NHC-Liganden unter Verwendung von $\mathrm{Pd}(\mathrm{OAc})_{2} \ldots \ldots \ldots \ldots \ldots$

2.7 In situ Deprotonierung am Beispiel eines Cr-NHC-Komplexes nach Öfele. . . . . . . . . . . . . . . . . 16

2.8 Deprotonierung eines NHC-Liganden i) unter Verwendung der externen Base $\mathrm{KO}^{t} \mathrm{Bu}$ und ii) mit $\mathrm{LiAlH}_{4}$. . . . . . . . . . . . . . 16

2.9 Deprotonierungsreaktion eines NHC mittels $\eta^{5}$-CyclopentadienylAnion. . . . . . . . . . . . . . . . 16

2.10 Umsetzung eines freien Carbens mit einem Metallvorläuferkomplex. 17

2.11 Darstellung eines Fe-NHC-Komplexes durch thermische Spaltung des entsprechenden Tetraaminoethylens mit Eisenpentacarbonyl. . . . . 17

2.12 Synthese des ersten Silber-NHC-Komplexes nach Arduengo. . . . . 18 
2.13 Mechanismus der Palladium-katalysierten allylischen Substitution.

2.14 Palladium-katalysierte allylische Substitution von Dimethylmalonat mit einem Diphenylallylsystem. . . . . . . . . . . . . . . 26

4.1 Synthese der Hydrochlorid-Liganden. . . . . . . . . . . . . . . . 30

4.2 Umsalzen der Liganden mittels Zugabe von $\mathrm{NH}_{4} \mathrm{PF}_{6}$ oder $\mathrm{NaBF}_{4}$. . 31

4.3 Synthese der Ag(I)-NHC-Komplexe. . . . . . . . . . . . . . . 42

4.4 Synthese von Palladium-NHC-Komplexen mittels Deprotonierung mit externer Base. . . . . . . . . . . . . . . . . . . . . . . . . . . 48

4.5 Synthese von Palladium-NHC-Komplexen unter Transmetallierung von Silber-NHC-Komplexen. . . . . . . . . . . . . . . . . . . . . 49

4.6 Mechanismus für eine $\eta^{3}-\eta^{1}-\eta^{3}$-Isomerisierung in einem Allylpalladiumkomplex. ............................. 52

4.7 Unterschiedliche Orientierung der Allylgruppen in Palladium-NHCKomplexen mit der Zusammensetzung $\left[\mathrm{L}^{\mathrm{x}} \mathrm{Pd}_{2}(\text { Allyl })_{2}\right]^{2+} \ldots$. . . .

4.8 Denkbare Synthese von Ni-NHC-Komplexen unter Transmetallierung von Silber-NHC-Komplexen mit $\mathrm{NiBr}_{2}$.DME. . . . . . . . . . .

4.9 Denkbare Synthese von Ni-NHC-Komplexen aus der Umsetzung vom Liganden mit Nickelocen. . . . . . . . . . . . . . . .

4.10 Palladium-katalysierte Addition von Dimethylmalonat an 1,3Diphenylallylacetat. . . . . . . . . . . . . . . . . 60

5.1 Diverse Strukturmotive der synthetisierten Silber(I)-NHC-Komplexe. 
Die hier vorgelegte Dissertation habe ich eigenständig und ohne unerlaubte Hilfe angefertigt. Die Dissertation wurde in der vorgelegten oder in ähnlicher Form noch bei keiner anderen Institution eingereicht. Ich habe bisher keine erfolglosen Promotionsversuche unternommen.

Maria Georgiou-Smith geb. Georgiou 


\section{Danksagung}

An erster Stelle möchte ich Herrn Prof. Dr. Franc Meyer für das interessante Thema und die Betreuung dieser Arbeit danken. Erst seine stete Diskussionsbereitschaft und Interesse an meiner Forschung ermöglichten es diese Dissertation anzufertigen.

Mein Dank gilt ebenfalls Herrn Prof. Dr. Dietmar Stalke für die freundliche Übernahme des Korreferats.

Viele Menschen haben mir auf unschätzbar wertvolle Weise bei dieser Arbeit zur Seite gestanden. Dr. Sebastian Dechert, Simone Wöckel und Vera Konstanzer möchte ich besonders für die Durchführung der Kristallstrukturanalysen danken.

Besonders zu Dank verpflichtet bin ich denjenigen, die mit mir zur gleichen Zeit im Arbeitskreis tätig waren: Ulrich J. Scheele, für die freundliche und kompetente Einführung in das neue Thema der Carben-Chemie, für die wertvollen Tipps und die vielen Diskussionen und Anregungen. Simone Wöckel munterte mich an den schlechten Tagen auf und schaffte es trotz ihres überfüllten Terminplans, Kristalle aufzusetzen und zu lösen. Simone Wöckel und Benjamin Schneider haben Teile meines Manuskripts aufmerksam gelesen und mir wertvolle Hinweise zu Veränderungen und Verbesserungen gegeben. Dr. Joanna „Asia“ Galezowska hat nicht nur das Labor mit mir geteilt, sondern ist auch eine gute Freundin geworden. Ich danke euch von Herzen!

Ferner möchte ich mich bei Jörg Teichgräber und Andreas Schwarz für die Synthese mancher Ausgangsverbindungen bedanken. Herzlichen Dank an die Mitarbeiter der NMR-Abteilung für die Aufnahme zahlreicher Spektren, insbesondere Dr. Michael John für die Hilfe bei den anspruchsvollen NMR-Experimenten und das Korrekturlesen meiner Veröffentlichungen. Auch gilt mein Dank den analytischen Abteilungen des Instituts für die Aufnahme von Massenspektren und Elementaranalysen. Annegret Boll (AK Steinem) möchte ich für die Aufnahme und Hilfestellung bei den Fluoreszenzspektren herzlich danken.

Ich möchte mich bei allen Forschungsstudenten bedanken, die an den experimentellen Arbeiten beteiligt waren: Anke Lemke, Jan-Hendrik Schütz und Sebastian Täubert.

Ich bedanke mich bei meinen Freunden, die mich während meiner Arbeit begleitet und mir geduldig zugehört haben - insbesondere aber bei Andrew ... 


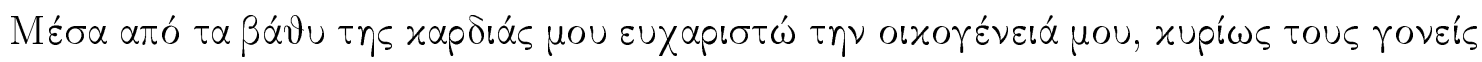

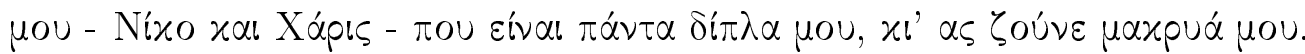

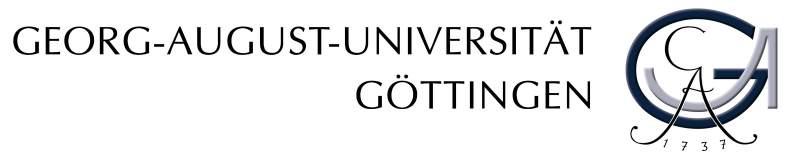




\section{Veröffentlichungen}

\section{Publikationen}

M. Georgiou, S. Wöckel, V. Konstanzer, S. Dechert, M. John, F. Meyer, „Structural Variations in Tetrasilver(I) Complexes of Pyrazolate-bridged Compartmental $\mathrm{N}$-Heterocyclic Carbene Ligands"

Zeitschrift für Naturforschung 2009, 64b, 1542-1552.

U. J. Schelle, M. Georgiou, M. John, S. Dechert, F. Meyer, „Combining Pyrazolate and $N$-Heterocyclic Carbene Coordination Motifs: Synthesis and Characterization of a Double-Crowned Silver Complex"

Organometallics 2008, 27, 5146-5151.

\section{Poster}

M. Georgiou, U. J. Scheele, S. Dechert, F. Meyer „Mehrkernkomplexe mit neuen Pyrazol/NHC-Hybridliganden“

5. Koordinationschemie-Treffen Februar 2009, Erlangen.

M. Georgiou, U. J. Scheele, S. Dechert, F. Meyer

„Synthese und Charakterisierung von Silberclustern und Palladiumkomplexen mit verbrückenden PyrazolNHC-Hybridliganden“

11. Norddeutsches Doktorandenkolloquium der Anorganischen Chemie September 2008, Burg Warberg, Braunschweig. 


\section{Lebenslauf}

\section{Persönliche Daten}

Name

Geboren

Nationalität
Maria Georgiou-Smith geb. Georgiou

17.11.1978 in Edessa, Griechenland

griechisch

\section{Hochschulausbildung}

11.2006-08.2010 Promotion: „Synthesewege zu neuen Mehrkernkomplexen von Pyrazol-NHCHybridliganden mit Übergangsmetallen - mit Fokus auf Silber-Komplexe“ betreut durch Prof. Dr. Franc Meyer im Institut für Anorganische Chemie an der Georg-August-Universität Göttingen

06. 2006

Erlangung das Akademischen Grads „Diplom-Chemiker“ mit der Gesamtnote "gut"

$012006-06.2006$

$10.1998-12.2005$

Diplomarbeit: „Verknüpfung von Metallocenen durch Hydrosilylierung“ betreut durch Prof. Dr. Christian Ganter im Institut für Anorganische Chemie I, Abteilung für Metallorganische Chemie der Heinrich-Heine-Universität Düsseldorf

09.2003

Studium der Chemie an der Heinrich-Heine-Universität Düsseldorf

Diplom-Vorprüfung mit der Gesamtnote „gut“

Vertiefungs- bzw. Spezialisierungsstudium:

„Stereochemische Untersuchungen zur nukleophilen Addition an

2-Methylferrocenylaldehyd“ am Institut für Anorganische Chemie I, Abteilung für Metallorganische Chemie,

Heinrich-Heine-Universität Düsseldorf, Prof. Dr. Christian Ganter

„Untersuchung der Löslichkeit von Titan in methanolischer Schwefelsäure“ am Max-Planck-Institut für Eisenforschung, Düsseldorf

Abteilung: Grenzflächenchemie und Oberflächentechnik, Prof. M. Stratmann

\section{Schulische Ausbildung}
$11.1993-06.1996$
„Nikolaos Oikonomidis“ Gymnasium, Edessa, Griechenland
$11.1990-06.1993$
Oberschule, Edessa, Griechenland
$11.1984-06.1990$
Grundschule, Edessa, Griechenland

Göttingen, den 07.10.2010 